ORNLTM-13286

OAK RIDGE NATIONAL LABORATORY
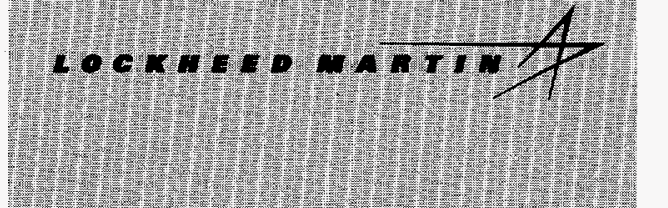

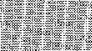

\section{位}

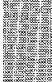

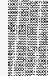

(4)

\section{(iti}

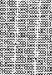

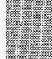

2.:-
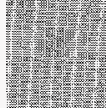

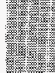

단.
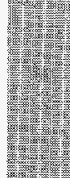

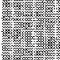

Vie.

15.

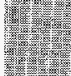

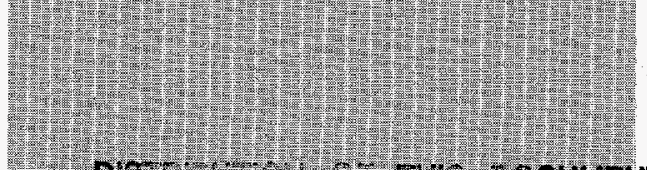

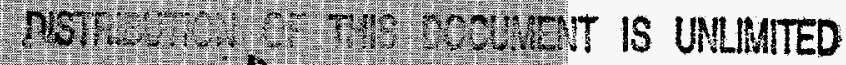

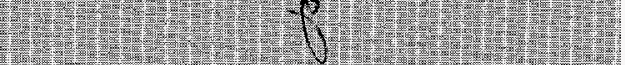

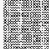

MWEED AD OPENMTEO DY

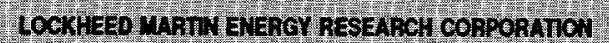
TORTEUTIEOSTIES DEPAMUET OF EALRG

\title{
Environmental Acceptability of High- Performance Alternatives for Depleted Uranium Penetrators
}

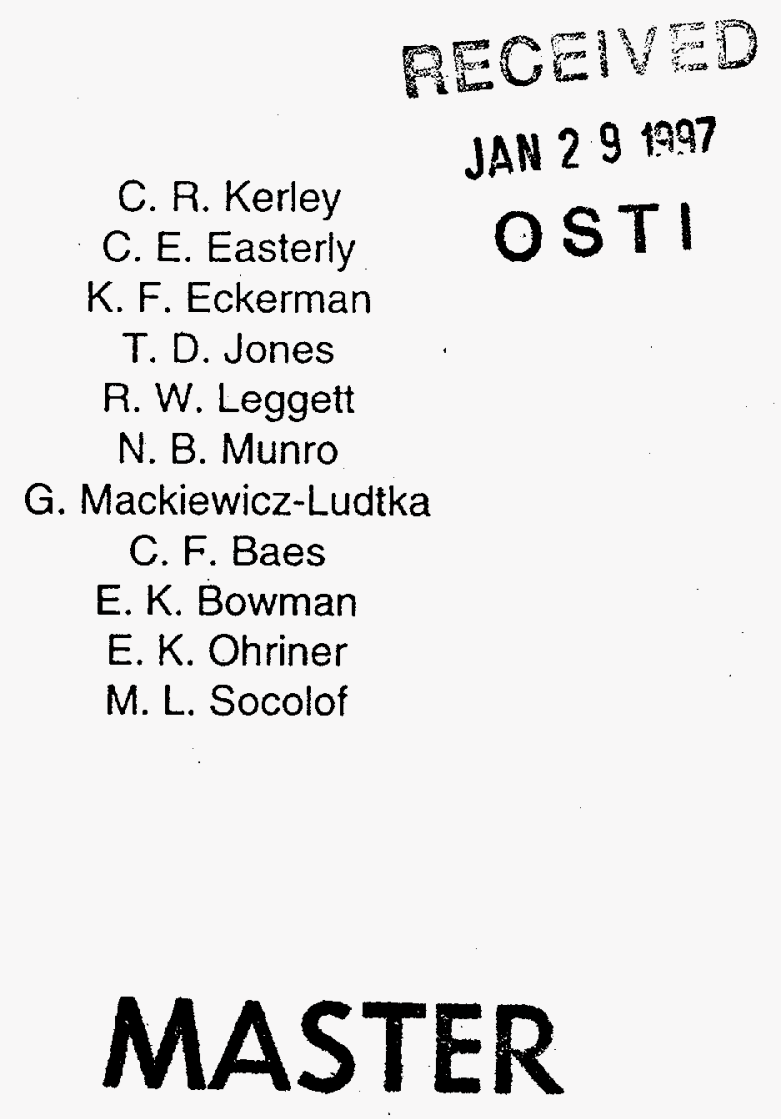


This report was prepared as an account of work sponsored by an agency of the United States Government. Neither the United States Government nor any agency thereot, nor any of their emptoyees, makes any warranty, express or implied, or assumes any legal liability or responsibility for the accuracy, completeness, or usefulness of any information, apparatus, product, or process disclosed, or represents that its use would not intringe privately owned rights. Reference herein to any specific commercial product, process, or service by trade name, trademark, manufacturer, or otherwise, does not necessarily constitute or imply its endorsement, recommendation, or favoring by the United States Government or any agency thereof. The views and opinions of authors expressed herein do not necessarily state or reflect those of the United States Government or any agency thereot. 
Environmental Acceptability of High-Performance Alternatives for Depleted Uranium Penetrators

$$
\text { by }
$$

\section{R. Kerley}

C. E. Easterly, K. F. Eckerman, T. D. Jones, R. W. Leggett, N. B. Munro,

G. Mackiewicz-Ludtka, C. F. Baes, E. K. Bowman,

E. K. Ohriner, M. L. Socolof

Date Published-August 1996

Prepared for

ARDEC, Piccatinny Arsenal

$$
\text { and }
$$

\section{SERRDP}

U.S. Department of the Army

Under Interagency Agreement DO No. 1892-A078-A1

Prepared by

Oak Ridge National Laboratory

Oak Ridge, TN 37831

managed by

LOCKHEED MARTIN ENERGY RESEARCH CORP.

for the

U.S. DEPARTMENT OF ENERGY

under Contract No. DE-AC05-96OR22464 


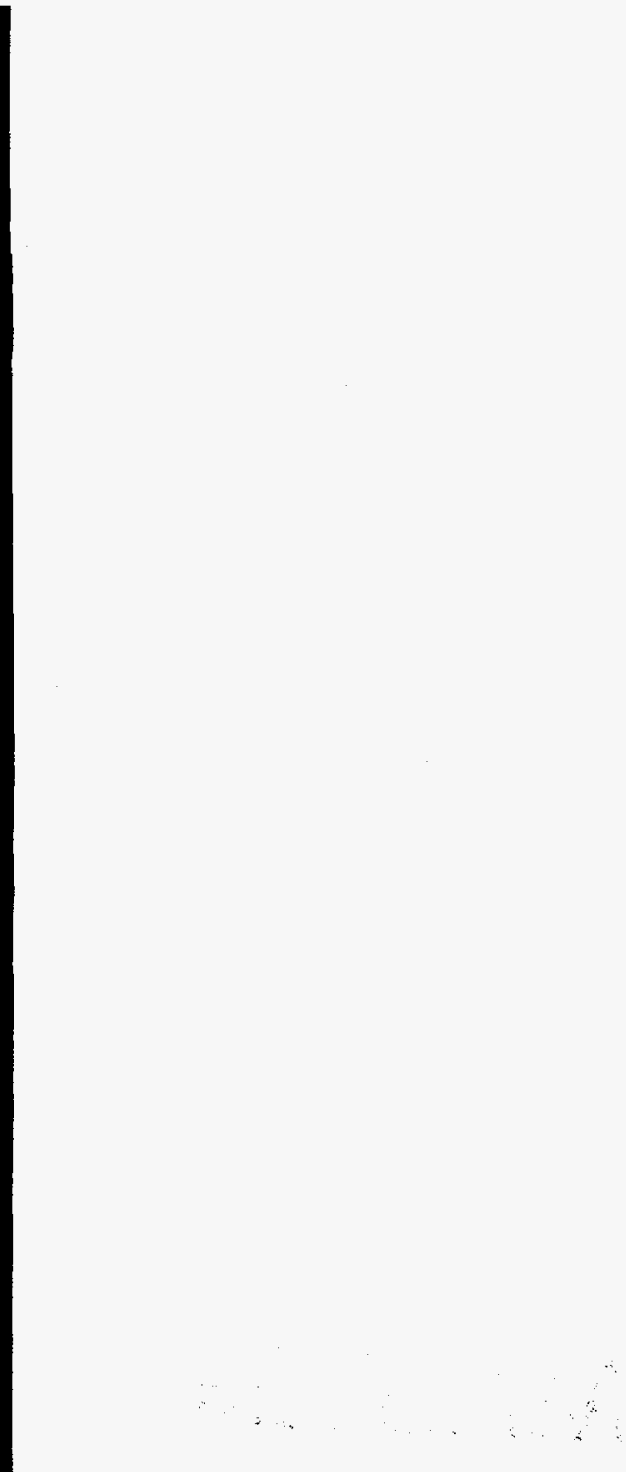




\section{DISCLAMMER}

Portions of this document may be illegible in electronic image products. Images are produced from the best available original document. 
LIST OF FIGURES $\ldots \ldots \ldots \ldots \ldots \ldots \ldots \ldots \ldots \ldots \ldots \ldots \ldots \ldots \ldots \ldots \ldots$ vii

LIST OF TABLES $\ldots \ldots \ldots \ldots \ldots \ldots \ldots \ldots \ldots \ldots \ldots \ldots \ldots \ldots \ldots \ldots \ldots \ldots \ldots$

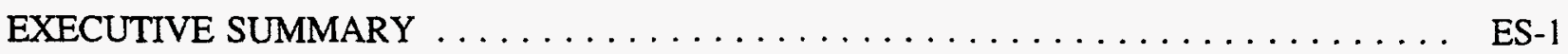

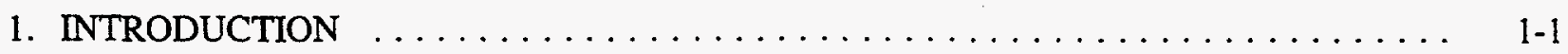

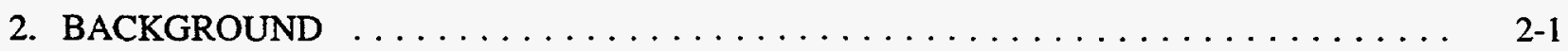

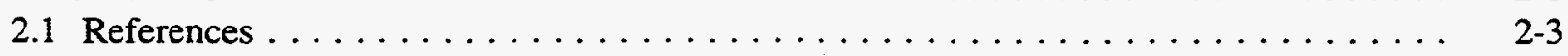

3. SCOPE OF WORK, APPROACH, AND METHODOLOGY $\ldots \ldots \ldots \ldots \ldots \ldots \ldots$ 3-1

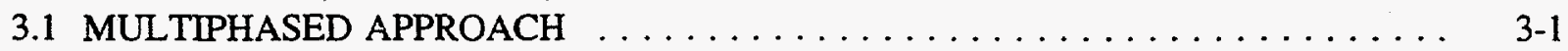

3.2 ANALYTICAL STRUCTURE $\ldots \ldots \ldots \ldots \ldots \ldots \ldots \ldots \ldots \ldots \ldots \ldots \ldots \ldots$ 3-1

3.2.1 Literature, Industry Interaction, and Model Simulation $\ldots \ldots \ldots \ldots \ldots \ldots$ 3-2

3.2.2 Preliminary Material Environmental Comparisons

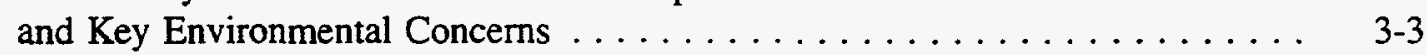

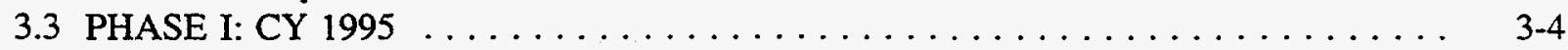

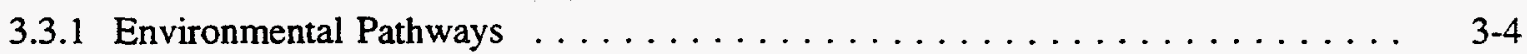

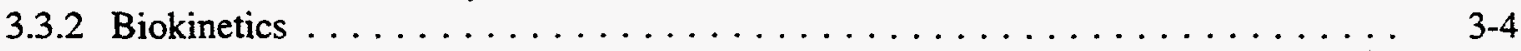

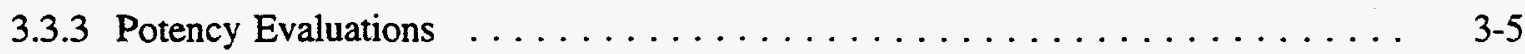

4. ENVIRONMENTAL TRANSPORT OF TUNGSTEN AND DU $\ldots \ldots \ldots \ldots \ldots \ldots$

4.1 ENVIRONMENTAL EXPOSURE CONCEPTUAL MODELS $\ldots \ldots \ldots \ldots \ldots$. 4 .1

4.2 NATURALLY OCCURRING TUNGSTEN $\ldots \ldots \ldots \ldots \ldots \ldots \ldots \ldots \ldots$ 4-3

4.3 ENVIRONMENTAL TRANSPORT AND TRANSFERS $\ldots \ldots \ldots \ldots \ldots \ldots \ldots$ 4-3

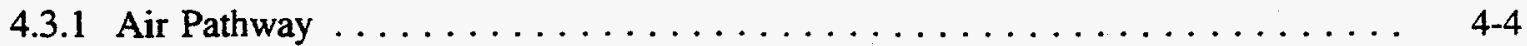

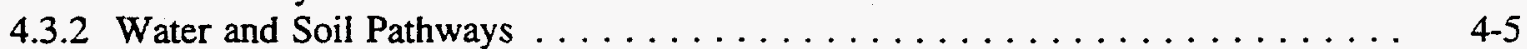

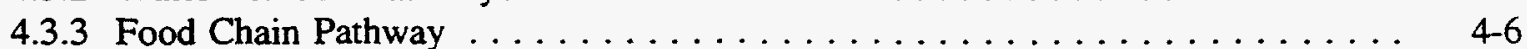

4.3.3.1 Soil-Plant Concentration Factor $\ldots \ldots \ldots \ldots \ldots \ldots \ldots \ldots \ldots$ 4-7

4.3.3.2 Terrestrial Animal (Livestock) Biotransfer Factor $\ldots \ldots \ldots \ldots \ldots$ 4-8

4.4 SUMMARY OF AVAILABLE TRANSFER DATA $\ldots \ldots \ldots \ldots \ldots \ldots \ldots \ldots .4 . \ldots \ldots$

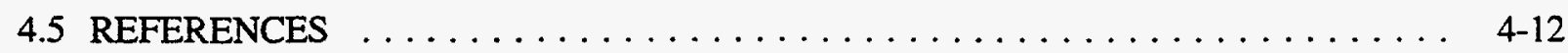

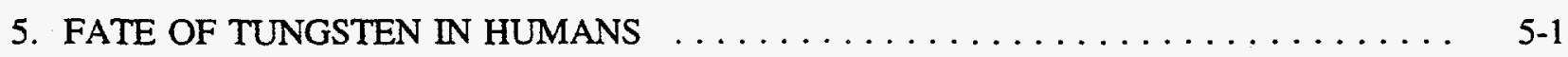

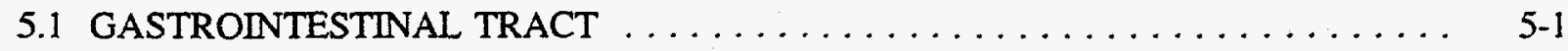

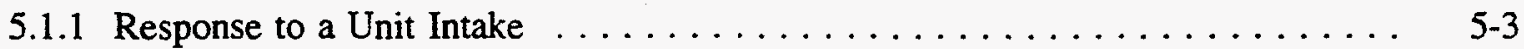

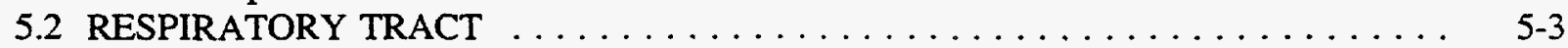

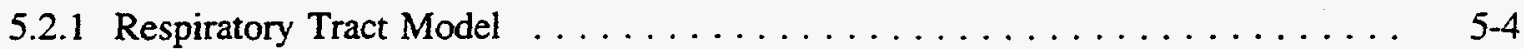

5.2.2 Deposition of Inhaled Particles $\ldots \ldots \ldots \ldots \ldots \ldots \ldots \ldots \ldots \ldots, 5-4$

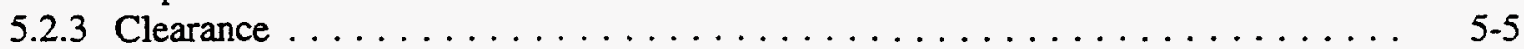




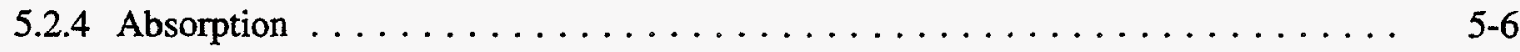

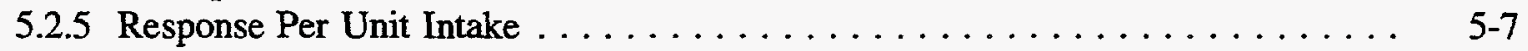

5.3 SYSTEMIC TUNGSTEN $\ldots \ldots \ldots \ldots \ldots \ldots \ldots \ldots \ldots \ldots \ldots \ldots \ldots$

5.4 TISSUE LEVELS FOLLOWING CHRONIC INTAKE $\ldots \ldots \ldots \ldots \ldots \ldots \ldots$

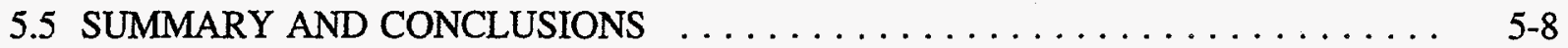

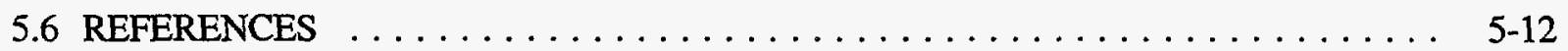

6. IMPLICATIONS OF TUNGSTEN TOXICITY FOR HUMANS $\ldots \ldots \ldots \ldots \ldots \ldots$

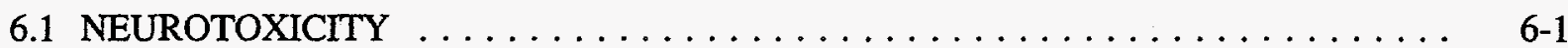

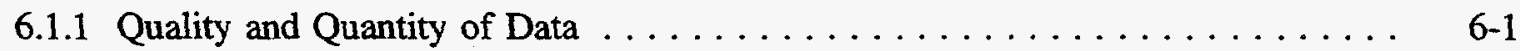

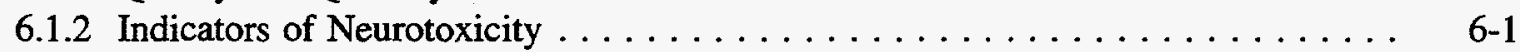

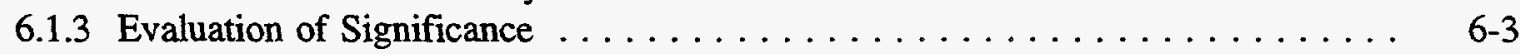

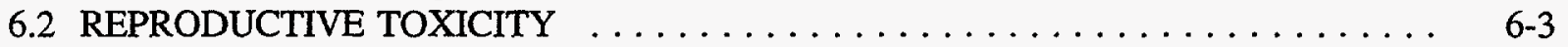

6.2 .1 Quality and Quantity of Data ....................... 6 6-3

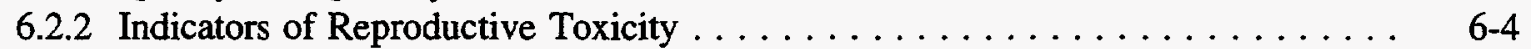

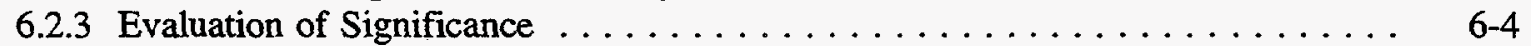

6.3 TERATOGENESIS $\ldots \ldots \ldots \ldots \ldots \ldots \ldots \ldots \ldots \ldots \ldots \ldots \ldots$

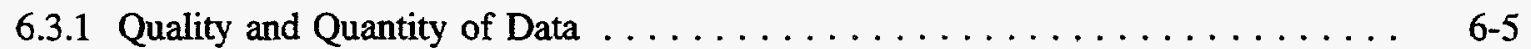

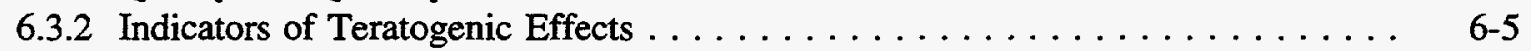

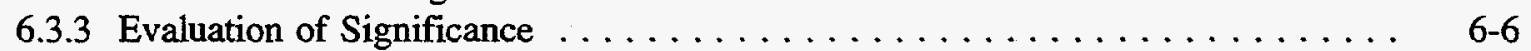

6.4 OTHER NONCANCER EFFECTS $\ldots \ldots \ldots \ldots \ldots \ldots \ldots \ldots \ldots \ldots \ldots \ldots$

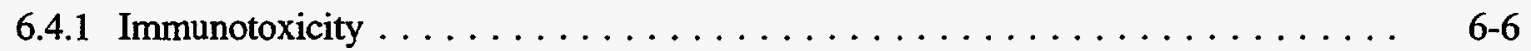

6.4 .2 Toxicity Enhancement $\ldots \ldots \ldots \ldots \ldots \ldots \ldots \ldots \ldots \ldots \ldots \ldots$ 6.6.

6.5 METHODS FOR ESTIMATING THE CARCINOGENIC POTENTIAL

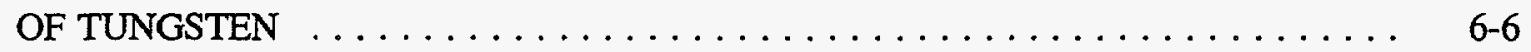

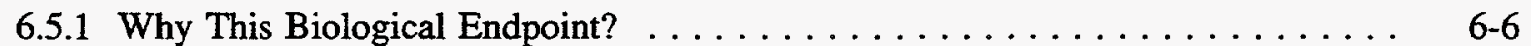

6.5.2 Cancer Potency Based on Relative Potency Considerations . . . . . . . . . 6-7

6.5.3 Practical Application-Comparison of Tungsten and DU . . . . . . . . . . 6-8

6.6 IMPLICATIONS OF PRELIMINARY FINDINGS $\ldots \ldots \ldots \ldots \ldots \ldots \ldots \ldots$. . . . $\ldots$. $\ldots$

6.7 SPECIFIC CONSIDERATIONS FOR DU IN LIGHT OF FINDINGS $\ldots \ldots \ldots \ldots$. . . 6-11

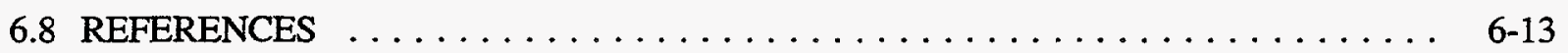

7. SIMULATION OF ENVIRONMENTAL EXPOSURE SCENARIO $\ldots \ldots \ldots \ldots \ldots \ldots$ 7-1

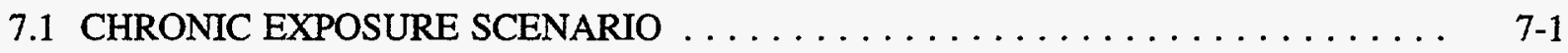

7.2 HUMAN HEALTH RESPONSES TO EXPOSURE SCENARIO . . . . . . . . . . 7-3

7.2 .1 Noncancer Toxicities . . . . . . . . . . . . . . . . . . $7-4$

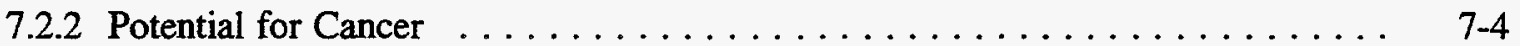

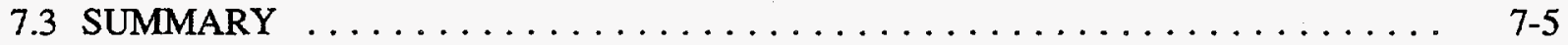

8. CONCLUSIONS, RECOMMENDATIONS, AND SUMMARY $\ldots \ldots \ldots \ldots \ldots \ldots$. $\ldots$

8.1 CONCLUSIONS FROM ENVIRONMENTAL SIMULATION MODELING RESULTS

8.1.1 Environmental Pathways $\ldots \ldots \ldots \ldots \ldots \ldots \ldots \ldots \ldots \ldots \ldots \ldots$ 8-1

8.1.2 Absorption, Retention, and Distribution Within the Human Body . . . . . . . 8 8-2

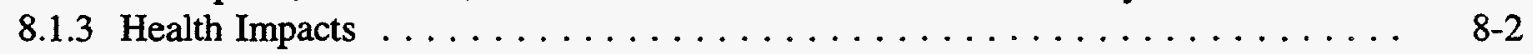

8.1.4 Summary of Phase I Accomplishments $\ldots \ldots \ldots \ldots \ldots \ldots \ldots \ldots \ldots \ldots$ 
8.2 RECOMMENDATIONS FOR FOLLOW-UP EXPERIMENTS AND TESTS $\ldots \ldots \ldots$. $8-3$

8.2.1 Environmental Pathways $\ldots \ldots \ldots \ldots \ldots \ldots \ldots \ldots \ldots \ldots \ldots$. 8.3

8.2.2 Absorption, Retention, and Distribution Within the Human Body . . . . . . . . 8-3

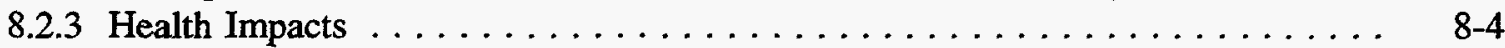

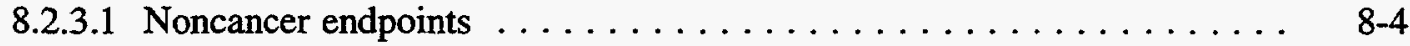

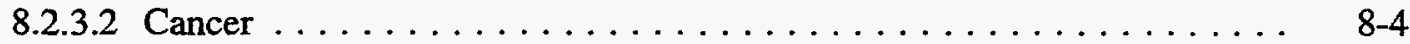

8.3 SPECIFIC CONSIDERATIONS FOR DU IN LIGHT OF FINDINGS $\ldots \ldots \ldots \ldots$

8.4 PRIORITIES FOR FILLING DATA GAPS $\ldots \ldots \ldots \ldots \ldots \ldots \ldots \ldots \ldots \ldots$

8.5 CONCLUSIONS FOR POLLUTION PREVENTION INITIATTVES $\ldots \ldots \ldots \ldots$

INTRODUCTION TO THE APPENDIXES $\ldots \ldots \ldots \ldots \ldots \ldots \ldots \ldots \ldots \ldots \ldots$ A-iii

APPENDIX A. BIBLIOGRAPHY ON TUNGSTEN WITH EMPHASIS ON

ENVIRONMENTAL INFORMATION $\ldots \ldots \ldots \ldots \ldots \ldots \ldots \ldots$ A-1

APPENDIX B: INDUSTRY AND INTERNATIONAL CONTACTS $\ldots \ldots \ldots \ldots \ldots$ B-1

APPENDIX C: BIOKINETIC MODEL FOR TUNGSTEN $\ldots \ldots \ldots \ldots \ldots \ldots \ldots \ldots$

APPENDIX D: COMPARISON OF LIMITS ON INTAKE OF URANIUM BASED

ON RADIOLOGICAL AND CHEMICAL GUIDANCE .......... D-1

APPENDIX E: COMPARISON OF THE BIOKINETICS OF TUNGSTEN AND

URANIUM IN ADULT HUMANS . . . . . . . . . . . . . . E-1

APPENDIX F: NONCANCER HEALTH EFFECTS $\ldots \ldots \ldots \ldots \ldots \ldots \ldots \ldots \ldots$ F-1

APPENDIX G: METHODS FOR ESTIMATING CANCER POTENCY OF

TUNGSTEN IN HUMANS $\ldots \ldots \ldots \ldots \ldots \ldots \ldots \ldots$ G-1 



\section{LIST OF FIGURES}

Figure

Page

4.1 Pictorial representation of environmental pathways $\ldots \ldots \ldots \ldots \ldots \ldots \ldots$

$4.2 \quad$ Detailed schematic of environmental pathways $\ldots \ldots \ldots \ldots \ldots \ldots \ldots$

$5.1 \quad$ Diagram of the GI tract model $\ldots \ldots \ldots \ldots \ldots \ldots \ldots \ldots \ldots \ldots \ldots \ldots$

5.2 Content of tungsten in various segments of the GI tract as a function

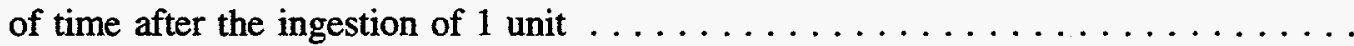

5.3 Fractional deposition in each region of the respiratory tract

for an adult as a function of the mass median aerodynamic diameter . . . . . . . .

5.4 Schematic diagram of movement of deposited particles in the

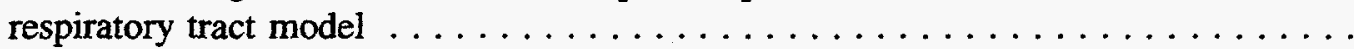

5.5 Absorption of tungsten and uranium following an acute intake of type $\mathrm{M}$ material

5.6 Content of tungsten and uranium in the kidneys of an adult human

as a function of the duration of chronic inhalation at a rate of $1 \mathrm{~g} \mathrm{~d}^{-1} \ldots \ldots \ldots$

5.7 Content of tungsten and uranium in the liver of an adult human

as a function of the duration of chronic inhalation at a rate of $1 \mathrm{~g} \mathrm{~d}^{-1}$

5.8 Content of tungsten and uranium in soft tissues other than kidneys and liver of an adult human as a function of the duration

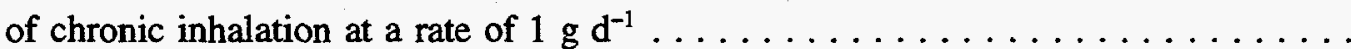

5.9 Content of tungsten and uranium in bone of an adult human as a function of the duration of chronic inhalation at a rate of $1 \mathrm{~g} \mathrm{~d}^{-1}$

6.1 Relative comparisons between tungsten and five reference compounds . . . . . . .

6.2 Preliminary analysis of tungsten in the context of EPA epidemiologically derived risk factors

C.1 Diagram of the respiratory tract model $\ldots \ldots \ldots \ldots \ldots \ldots \ldots \ldots \ldots$

C.2 Diagram of the gastrointestinal tract model $\ldots \ldots \ldots \ldots \ldots \ldots \ldots$

C.3 Diagram of the biokinetic model for systemic tungsten $\ldots \ldots \ldots \ldots \ldots$ C-8 
C.4 Diagram of the biokinetic model for systemic tungsten used in this report

C.5 Data on retention of tungsten in kidneys of laboratory animals and predictions of the present model for the case of intravenous injection of tungsten into a typical adult human

C.6 Data on retention of tungsten in livers of laboratory animals and predictions of the present model for the case of intravenous

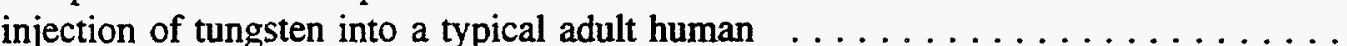

C.7 Data on retention of tungsten in bone of laboratory animals and predictions of the present model for the case of intravenous

injection of tungsten into a typical adult human

D.1 Diagram of the biokinetic model for systemic uranium

D.2 Comparison of limits on intake of uranium by chronic inhalation based on radiological (Rad) and chemical (Chem) guidance for different isotopic mixtures and absorption types

D.3 Comparison of limits on acute inhalation of uranium based on radiological (Rad) and chemical (Chem) guidance for different isotopic mixtures and absorption types

D.4 Comparison of limits on intake of uranium by chronic ingestion based on radiological (Rad) and chemical (Chem) guidance for different isotopic

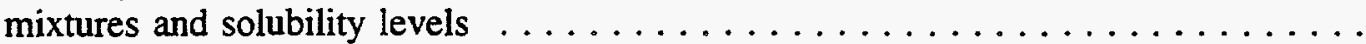

D.5 Comparison of limits on acute ingestion of uranium based on radiological (Rad) and chemical (Chem) guidance for different isotopic mixtures and solubility levels

E.1 Comparison of content of tungsten and uranium in the skeleton of an adult human as a function of time after injection, as predicted by the biokinetic models used in this report

E.2 Comparison of content of tungsten and uranium in the kidneys of an adult human as a function of time after injection, as predicted by the biokinetic

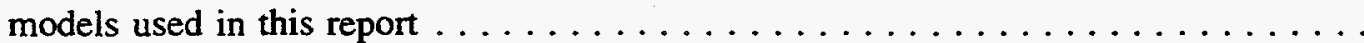

E.3 Comparison of content of tungsten and uranium in the liver of an adult human as a function of time after injection, as predicted by the biokinetic models used in this report

E.4 Comparison of content of tungsten and uranium in other soft tissues of an adult human as a function of time after injection, as predicted by the biokinetic models used in this report 
E.5 Comparison of content of tungsten and uranium in the skeleton of an adult human as a function of time after inhalation of type $\mathrm{F}$ material, as predicted by the biokinetic models used in this report $\ldots \ldots \ldots \ldots \ldots \ldots \ldots \ldots$

E.6 Comparison of content of tungsten and uranium in the kidneys of an adult human as a function of time after inhalation of type F material, as predicted by the biokinetic models used in this report.

E.7 Comparison of content of tungsten and uranium in the skeleton of an adult human as a function of time after inhalation of type $M$ material, as predicted by the biokinetic models used in this report $\ldots \ldots \ldots \ldots \ldots$

E.8 Comparison of content of tungsten and uranium in the kidneys of an adult human as a function of time after inhalation of type $M$ material, as predicted by the biokinetic models used in this report

E.9 Comparison of content of tungsten and uranium in the skeleton of an adult human as a function of time after inhalation of type $\mathrm{S}$ material, as predicted by the biokinetic models used in this report

E.10 Comparison of content of tungsten and uranium in the kidneys of an adult human as a function of time after inhalation of type $S$ material, as predicted by the biokinetic models used in this report $\ldots \ldots \ldots \ldots \ldots$

E.11 Comparison of content of tungsten and uranium in the skeleton of an adult human as a function of time after ingestion of soluble material, as predicted by the biokinetic models used in this report $\ldots \ldots \ldots \ldots \ldots$

E.12 Comparison of content of tungsten and uranium in the kidneys of an adult human as a function of time after ingestion of soluble material, as predicted by the biokinetic models used in this report

G.1 Comparison of the $\mathrm{TD}_{50}$ (i.e., the does that reduces the number of tumor-free animals by $50 \%$ ) in mice vs the $\mathrm{TD}_{50}$ in rats for the 33 compounds in the list of 101 for which positive test results were obtained in both mice and rats . . . . . .

G.2 Comparison of position in the periodic chart for transition metals that may be used as a reference for the toxicity of tungsten $\ldots \ldots \ldots \ldots \ldots$

G.3 Stages in carcinogenesis as described by Kouri, Henry, and Kriesher (1980) . . . . . . . . . . . . . . . . . . . . . . .

G.4 Conceptualized schematic to illustrate the subjective boundaries within the toxicological continuum

G.5 Hierarchial rank of different data bases considered to be biologically relevant to tumorigenesis and human risks $\ldots \ldots \ldots \ldots \ldots \ldots \ldots \ldots \ldots \ldots \ldots \ldots$ 
G.6 Variability of different bioassay methods and extrapolation models $\ldots \ldots \ldots$. . G-22

G.7 Chemicals having independent mechanisms of action lead to risk-additive models while chemicals having joint action on a particular response or mechanism lead to dose-additive models

G.8 Data from many chemical, physical, and biological models can be used for hazard assessment

G.9 Scatter plots of the potency of 67 compounds relative to 5 different reference compounds taken two at a time

G.10 Median relative potency values from Table G.5 were compared with the $\mathrm{TD}_{50}$ estimates from Gold et al. (1989) and the maximum doses tested in the 2-year rodent carcinogenesis tests

G.11 Cumulative log-normal frequency plots for potency-adjusted to $\mathrm{TD}_{50}$ doses (gold et al. 1989) and maximum does tested (Gold et al. 1984)

G.12 Graphical comparisons of potencies completed in Table G.9 . . . . . . . . . G-48

G.13 From the few common bioassays for sodium tungstate and sodium chromate (VI), it appears that the median potency of sodium tungstate is about $20 \%$ that of sodium chromate $\ldots \ldots \ldots \ldots \ldots \ldots$ 


\section{LIST OF TABLES}

Table

Page

4.1 Intake and consumption rates of exposure media $\ldots \ldots \ldots \ldots \ldots \ldots \ldots$

4.2 Tungsten CFs derived from a review of the literature $\ldots \ldots \ldots \ldots \ldots$

4.3 Tungsten BTFs derived from the literature $\ldots \ldots \ldots \ldots \ldots \ldots \ldots \ldots$

4.4 Comparison of tungsten and $U$ transfer coefficients $\ldots \ldots \ldots \ldots \ldots \ldots \ldots$

5.1 Fractional absorption of selected elements $\ldots \ldots \ldots \ldots \ldots \ldots \ldots \ldots$

$5.2 \quad$ Parameters of the default absorption types $\ldots \ldots \ldots \ldots \ldots \ldots \ldots$

5.3 Tungsten and uranium content of body organs $(\mathrm{g})$ assuming chronic intake

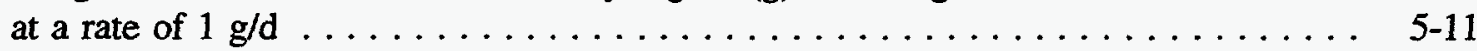

$6.1 \quad$ Evidence for tungsten's possible noncancer toxicity $\ldots \ldots \ldots \ldots \ldots \ldots \ldots \ldots$

7.1 Tungsten and depleted uranium levels in the body resulting

from chronic exposure to $10.0 \mathrm{ppm}$ of tungsten and DU in soil

D.1 Transfer rates $\left(\mathrm{d}^{-1}\right)$ for the systemic biokinetic model for tungsten $\ldots \ldots \ldots \ldots$ D-7

F.1 Regulations and guidelines for workplace exposures to soluble and

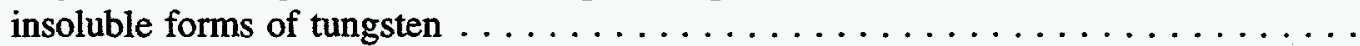

F.2 Acute toxicity data for several tungsten compounds $\ldots \ldots \ldots \ldots \ldots \ldots \ldots$

F.3 Partial list of chronic toxicity data for tungsten $\ldots \ldots \ldots \ldots \ldots \ldots \ldots$

F.4 Neurotoxicity of tungsten and its compounds in animals $\ldots \ldots \ldots \ldots \ldots \ldots$ F-7

F.5 Levels of tungsten in rat tissues resulting from unit ingestion exposure $\ldots \ldots \ldots$ F-11

F.6 Levels of tungsten in human soft tissues resulting from unit ingestion exposure $\ldots \ldots$ F-11

F.7 Reproductive toxicity of tungsten $\ldots \ldots \ldots \ldots \ldots \ldots \ldots \ldots \ldots \ldots \ldots \ldots$

F.8 Developmental toxicity of tungsten and molybdenum $\ldots \ldots \ldots \ldots \ldots \ldots \ldots \ldots$ F-21

G.1 Summary of effects data from substances usually considered biologically inert . . . G G-15

G.2 Summary of bioassay results for testing/screening of hafnium, tungsten, and zirconium-related compounds 
G.3 Hazard index examples showing that rad-dose $\mathrm{R}$ and chem dose $\mathrm{C}$ may be based on doses ri and ci, which induce the same level

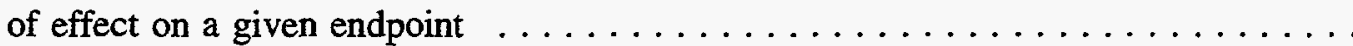

G.4 Potency values for 67 compounds relative to $B(a) P$, nicotine, cisplatin, aflatoxin $B_{1}$, and cyclophosphamide

G.5 Common scales of toxicological potency for 67 compounds based on RPs to five reference compounds from Table G.4 .

G.6 Fitted values for a log-normal frequency distribution function comparing potency-adjusted $\mathrm{TD}_{50}$ and maximum doses tested

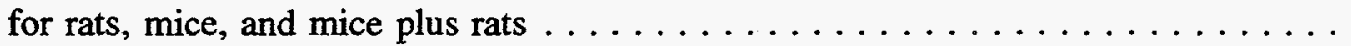

G.7 Bioassay-specific radiation dose-response values for the denominator

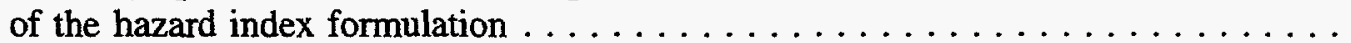

G.8 Toxicological test data for sodium chromate (VI) [RTECS \#GB2955000] $\ldots \ldots \ldots$

G.9 Summary of the potency of four tungsten compounds and sodium chromate relative to $B(a) P$, cyclophosphomide, nicotine, aflatoxin $B_{1}$, and cisplatin all converted to a common scale indexed by unit potency for $\mathrm{B}(\mathrm{a}) \mathrm{P}$; cyclophosphomide, nicotine, aflatoxin, and cisplatin were standardized according to potencies listed at the bottom of the table

G.10 Bioassay test data for tungsten [YO7175000], tungsten oxides [YO7760000], sodium tungstate [YO7875000], and tungstic acid, sodium salt, dihydrate [YO7900000] 


\section{EXECUTIVE SUMMARY}

This document summarizes the results from the first phase (Phase I) of a proposed three-year effort to evaluate the environmental acceptability of tungsten (W) as an alternative to depleted uranium (DU) in kinetic energy penetrators (KEPs). The objective of this study was to evaluate the environmental impacts of tungsten in a military context in response to military and congressional concerns regarding exposures to DU. The primary purposes of this Phase I study were to review the literature for existing data; to formulate a test methodology that will provide a useful life cycle assessment of the environmental impacts of tungsten; and to identify where the data gaps exist. In this initial effort, the environmental pathways by which tungsten chemical species (from penetrator impact fragments and aerosols), deposited on the soil, might find their way into humans were identified. In addition, a new computer-based modeling tool was developed which enables predictions of the ways tungsten is retained and distributed in the body. The toxic effects which might be produced were addressed. This approach can be applicable to other materials and, in fact, provides a useful tool for comparing the relative environmental impacts expected from numerous, alternative materials.

An extensive review of the literature reveals that little quantitative information and data are available to permit reliable simulations of tungsten toxicity in humans. In particular, the relative corrosion kinetics of each metal, the relative solubilities in corrosive media, and the relative rate of diffusion of the species through corrosive media under comparable experimental conditions are not readily available. Like most metals, the corrosion behavior of DU is quite complex and strongly dependent on the experimental and environmental conditions. The data base describing the corrosion of DU is more extensive than the one for tungsten. Furthermore, there is a severe lack of direct, one-to-one quantitative data from all sources directly comparing DU and tungsten. Hence, all simulations described in this report are not absolute and are not intended to be used for decision-making in assessing the relative environmental and health hazards/risks associated with $D U$ or tungsten.

The simulations described in this report have been based on the limited, unreplicated test results reported in various independent studies. The biological behavior and toxic effects of tungsten in humans have received little attention in previous analyses and present great uncertainties in any characterization of environmental impacts of tungsten. While numerous bits and pieces of information on the biological behavior and toxic effects of tungsten were found in the literature, little effort has been made previously to synthesize this information within a biologically meaningful framework. In this study, the available fragmented and disparate data on the biokinetics and potential toxic effects of tungsten were collected, analyzed, and integrated using state-of-the-art biochemical and biokinetic methodologies developed at Oak Ridge National Laboratory. In the absence of the necessary data, this approach provides the most reasonable basis for estimating the environmental effects of tungsten that is currently possible. Several simplifying, qualitative assumptions were made in order to (1) demonstrate the availability and potential value of the simulation tools developed in predicting the potential environmental and health impacts and (2) suggest whether there are any indications of differences in environmental mobility and potential health effects related to the retention of tungsten vs DU. We have identified clear needs for more data and have concluded that there is an inadequate data supply to support important decisions regarding the relative environmental impacts of tungsten $v s D U$.

Although we have attempted to indicate and compare the relative potential for hazards from tungsten and DU, a detailed investigation of potential health effects of uranium was outside the scope of this 
Phase I study. In the present study, considerations of the toxic effects of uranium were limited to those addressed in current federal guidance for exposure to uranium. These effects are the toxicity to the kidneys and the theoretical carcinogenic potential of uranium from its radioactivity. There is little information on other, more subtle health effects of DU in chronically exposed humans. Both DU and tungsten are heavy elements and might be suspected of having biological effects similar to those of other heavy elements. Studies on laboratory animals suggest potential developmental and reproductive effects for both DU and tungsten.

To help keep the potential hazards of tungsten and uranium in perspective, it should be noted that industrial experiences with these two elements suggest that both may have a relatively low order of toxicity. On the other hand, the available data on uranium and tungsten workers may not reveal relatively subtle health effects of either element on persons exposed, particularly if there is a long delay in the expression of these effects. Moreover, unlike the historical uranium industry work force, the general population has a substantial proportion of individuals (e.g., pregnant women and children) who might be more sensitive to the noncancer biological effects highlighted within this report. Such effects are teratogenesis, developmental, neurological, and reproductive disorders. In addition, some of the toxicity data available for tungsten is tainted by the presence of other elements, such as cobalt, that already have been linked to toxic effects.

When comparisons from simulations and scenarios have been made in this report, it is important to notice that these are based on the simplifying assumptions (except where noted) about amounts of DU and tungsten that are available for a particular receptor. Also, it is assumed that the material would be available in a chemical form that would be soluble in the receptor and hence available for potential environmental impacts. However, observations of DU and tungsten under ambient conditions suggest large differences in chemical reactivity and oxidation behavior. In general, such data suggest DU to be far more reactive than tungsten (Uhlig 1955, Fischer 1960, Leibowitz et al. 1961, Ryle et al. 1960).

Because of the unavailability of one-to-one data comparisons between DU and tungsten and the simplifying assumptions (made in this report) that tungsten and DU are available, the comparisons of the relative hazards associated with tungsten and uranium are qualitative indicators only. Despite the paucity of data, and the simplifying assumptions made, simulations have been run to indicate the relative qualitative hazards. These results indicate that for some chemical forms of tungsten the following potential concerns may exist: (1) tungsten appears to have a higher mobility in plants and some soils compared to DU; (2) tungsten appears to have higher access to systemic regions in the body than DU that may not be completely negated by tungsten's higher excretion rate; and (3) tungsten and DU have not been tested adequately to determine their potential with respect to cancer and noncancer toxicity. The purpose of the proposed Phase $\Pi$ of this program is to generate the data and information needed to provide an actual assessment of the relative quantitative hazards associated with tungsten and DU.

Generating meaningful results from computer simulations or parametric studies necessitates input data that have been obtained from well-documented and controlled experiments. Reliable predictions of the relative health and environmental impacts associated with tungsten as an alternative material to DU requires high-quality input data. The types of information and data needed are (1) the identity of the prominent, chemical species or forms that are present in the environment and available; (2) the relative size, amount, and distribution of these species; (3) their relative solubilities in particular receptors 
(water, blood, etc.); (4) the transport of these species in these receptors; and (5) the biological response to relevant quantities of transported species.

In summary, although these analyses indicate that tungsten's environmental behavior and health effects may not be as benign as previously assumed, DU may also have been inadequately studied for both cancer and noncancer toxicities. Data available for both tungsten and DU are insufficient for choosing between them from an environmental and health stand point. Additional data are necessary for both tungsten and DU to ensure that future comparisons can be made on a more nearly common basis. Descriptions of our future directions to carry out this intention are found within the body of the report and, in detail, in the Appendixes.

\section{REFERENCES}

Uhlig, H. H. 1955. The Corrosion Handbook, Wiley and Sons, N.Y.

Fischer, R. B. 1960. "Tungsten," in Reactor Handbook, Vol. I, ed. C. R. Tipton, Jr., Interscience Publishers, Inc., N.Y.

Leibowitz, L., et al. 1961. "The Kinetics of Oxidation of Uranium Between $125^{\circ} \mathrm{C}$ and $250^{\circ} \mathrm{C}$," J. Electrochem. Soc. 108, 1155-60.

Ryle, B. G., et al. 1960. "Uranium," in Reactor Handbook, Vol. I, ed. C. R. Tipton, Jr., Interscience Publishers, Inc., N.Y. 


\section{INTRODUCTION}

The Army's environmental program is organized around four major goals: (1) conservation is the management of Army lands to ensure long-term natural resource productivity and to balance the need for long-term resource use and resource protection; (2) compliance ensures that current operations at Army installations and civil project sites meet federal, state, local, and applicable host-nation environmental requirements and Army regulations; (3) restoration activates cleanup of contaminated sites; and (4) prevention focuses on eliminating pollution to the greatest extent possible by reducing hazardous materials use and hazardous waste generation. The Army's environmental strategy for investigating material substitution and management is to measure system environmental gains/losses in all phases of the material management life cycle from cradle to grave. This study is the first in a series of new investigations, applying material life cycle concepts, to evaluate whether there are environmental benefits from increasing the use of tungsten as an alternative to depleted uranium (DU) in Kinetic Energy Penetrators (KEPs).

Current military armor penetrators use DU and tungsten as base materials. Although DU alloys have provided the highest performance of any high-density alloy deployed against enemy heavy armor, its low-level radioactivity poses a number of environmental risks. These risks include exposures to the military and civilian population from inhalation, ingestion, and injection of particles. Depleted uranium is well known to be chemically toxic (kidney toxicity), and workplace exposure levels are based on its renal toxicity. Waste materials containing DU fragments are classified as low-level radioactive waste and are regulated by the Nuclear Regulatory Commission. These characteristics of DU do not preclude its use in KEPs, given the thorough management procedures that are being implemented by the Army. However, long-term management challenges associated with KEP deployment and improved public perceptions about environmental risks from military activities might be well served by a serious effort to identify, develop, and substitute alternative materials that meet performance objectives and involve fewer environmental risks. Tungsten, a leading candidate base material for KEPs, is potentially such a material because it is not radioactive. Tungsten is less well studied, however, with respect to health impacts and other environmental risks. The present study is designed to contribute to the understanding of the environmental behavior of tungsten by synthesizing available information that is relevant to its potential use as a penetrator.

The report is organized into eight chapters and seven appendixes. In Chapter 2, background information is presented on the performance characteristics of materials that make them useful in KEPs. Chapter 3 describes the methodologies used in this study to address the environmental potency of tungsten compared to DU. The environmental mobility of tungsten and comparisons to DU are presented in Chapter 4. In Chapter 5, model simulations of the biokinetic behavior of tungsten in comparison to DU in humans is documented. This work represents the first stage of sorting out the common and different bioreceptor sites at which to look for long-term health responses from retained penetrator materials. Chapter 6 examines relevant toxicological data for application to humans for neurological, reproductive, and developmental toxicity effects. This chapter also presents modeling results for determining possible cancer potency. Chapter 7 provides an initial look at the types of simulations that will be possible in later phases of this program. The investigators have provided an analysis of a single scenario involving both the inhalation and the ingestion pathways. This work demonstrates that sufficient pieces of information and models have been assembled to conduct a 
preliminary quantitative environmental assessment of tungsten as a penetrator material and to identify the most critical data gaps.

Chapter 8 presents major conclusions of this first phase and a discussion of some of the potential consequences of the conclusions, as well as uncertainties that can only be removed with additional data. This chapter also highlights brief descriptions of data gaps and additional biological studies that are considered to be important in either validating data that require replication or in providing necessary confirmation of trends. 


\section{BACKGROUND}

Tungsten is used in materials for armor penetration because of its high density of $19.3 \mathrm{~g} / \mathrm{cc}$. In many $\mathrm{KEP}$ applications, density is of primary importance because it is directly proportional to the quantity of kinetic energy per unit area that is available to defeat the target. In these applications, tungsten is one of a very limited number of high-density materials that can be considered for use. Tungsten is the most dense metal, with the exception of the much rarer element rhenium and four precious metals, gold, platinum, iridium, and osmium. The only two other elements that have densities above $16 \mathrm{~g} / \mathrm{cc}$ are tantalum at 16.6 and uranium at $18.9 \mathrm{~g} / \mathrm{cc}$. The cost of tantalum is about 10 times greater than that of tungsten. The costs of the high-density precious metals are roughly a factor of 100 greater than tungsten. As a practical matter, only tungsten and DU have the combination of availability, cost, and the high density needed for these armor penetrating applications.

Although armor penetration in general requires a high density for increased penetration performance, there are a range of other property requirements that include those important to the penetration event itself, those related to launching of the penetrator, and those related to manufacturing and storage. The property requirements can vary for different types of targets and different weapon systems.

Sintered tungsten alloys have been used for many years for armor penetrating applications. The alloys typically contain 90 to $97 \%$ tungsten by weight. The remainder of the material is a binder that consists mostly of nickel, with lesser amounts of iron and/or cobalt. These materials are produced by blending elemental powders, pressing the powders to a specific shape, and sintering them at a temperature above the melting point of the binder but well below that of tungsten. The alloys have densities lower than that of pure tungsten but have properties not possessed by pure tungsten. The alloys can be processed much more easily than pure tungsten because much lower processing temperatures can be used. Unlike pure tungsten, the alloys are readily machinable. The alloys also have much greater ductility and toughness than pure tungsten.

DU is used as an alloy for armor penetrators because of its high density and ready availability as a low-cost byproduct of uranium enrichment operations. The most common alloy in use is the uranium $(0.75 \%)$ /titanium (by weight) material. This alloy has the best combination of strength and ductility of the uranium alloys and has moderate corrosion resistance (ASM International 1990).

The penetration performance of tungsten alloys vs uranium alloys depends on the type of penetrator used, the nature of the target, and a range of system considerations. Tungsten materials are uniquely well suited to many applications of small calibre penetrators and penetrators with a low ratio of $\mathrm{L} / \mathrm{D}$ (length to diameter) (Dowding et al. 1994). Values of L/D less than 8 are representative of those used against light armor. The materials properties that are important in this type of application are strength, toughness, and stiffness. These properties become important in the case of an oblique impact of the penetrator with the target and for multiple impacts with a target. In these types of applications the performance of tungsten alloys is found to be equivalent or superior to that of DU alloys. For instance, in penetrators used to defeat anti-ship missiles, the tungsten alloy has been found to be superior to that of the best DU alloy and is replacing the production DU round previously used. 
Penetrators with large L/D ratios are needed to obtain the high energy density necessary to defeat heavy armor. Values of $\mathrm{L} / \mathrm{D}$ of 15 and higher, are used in these applications. Although materials properties such as strength, ductility, and toughness remain important to overall design, including the launch of the penetrator and its performance against oblique and spaced targets, the controlling factor in the penetration through thick armor is the deformation behavior of the penetrator material under conditions of high compressive stresses, high strain rates, and rapid generation of heat. Under these circumstances, DU outperforms tungsten alloys. Penetration tunnels seen in thick "semi-infinite" homogeneous steel targets show that uranium alloys produce a more narrow tunnel through the steel than do tungsten penetrators of the same dimensions. This is caused by a mushroom shape produced at the head of the tungsten alloy as it moves through the armor. The uranium alloy, in contrast, maintains a point shape at the head of the penetrator to produce a narrower and deeper hole. This behavior is associated with a different mechanism of the deformation of the uranium alloy known as adiabatic shear, a phenomenon that is only partially understood and that does not correlate with any normal engineering measurements of strength or ductility. In addition, the relative performance of uranium and tungsten alloys in penetration of steel has been shown to be a strong function of the impact velocity (Magness 1993). At impact velocities in the range of 100 to $1300 \mathrm{~m} / \mathrm{s}$, the penetration depth of the tungsten alloy is on the order of $15 \%$ below that of the uranium $(0.75 \%)$ /titanium alloy. As impact velocities increase, the relative performance of tungsten alloy improves, and at $1700 \mathrm{~m} / \mathrm{s}$ the difference in penetration depths is less than 5\%. At this velocity or higher, the mechanical properties of strength, stiffness, ductility, and toughness are again of primary importance, and the existing tungsten alloys or some small modifications of these materials could produce a performance superior to that of the uranium alloy materials.

The demonstration of a tungsten alloy penetrator with penetration performance equivalent to that of the uranium alloy at impact velocities near $1300 \mathrm{~m} / \mathrm{s}$ has been a goal of Army-sponsored research for several years. Although considerable progress has been made, the goal has not yet been achieved. Consequently, it is not possible to state what composition such a tungsten alloy might have. It is reasonably certain that the tungsten content would be at least $90 \%$ by weight in order to achieve the necessary overall density of the alloy. The alloying elements that are currently under most active investigation include hafnium, titanium, iron, nickel, and aluminum. A number of other alloying elements, including manganese, cobalt, and zirconium, are also potential candidates.

An extensive review of the available literature reveals a severe lack of direct, one-to-one quantitative data directly comparing the corrosion and oxidation characteristics of DU and tungsten. The relative corrosion kinetics of each metal, their relative solubilities in the corrosive media, and the relative diffusion rate of the species through such media under comparable experimental conditions are not readily available. The specific types of data necessary to permit reliable simulations are (1) the identity of the prominent, chemical species or forms that are present and available; (2) the relative size, amount, and distribution of these species; (3) the relative quantities of the species available; (4) the relative chemical stability of these species; (5) their relative solubilities in particular receptors (water, blood, etc.); (6) the transport of these species in these receptors; and (7) the biological response to relevant quantities of transported species.

Although there is quite a bit of information available on DU and its corrosion behavior, there is relatively little for tungsten. Like corrosion of so many materials, the corrosion behavior of DU is quite complex and is strongly dependent on the experimental conditions under which it was evaluated. Consequently, comparisons of the relative hazards associated with tungsten and uranium are qualitative indicators only. When comparisons have been made in this report, the simplifying assumptions have 
been (except where noted) that equal amounts of $\mathrm{DU}$ and tungsten are available for a particular receptor and that all of each material would be available in a chemical form that would be soluble in the receptor.

Qualitatively, experimental observations on DU and tungsten under ambient conditions seem to suggest that the characteristics and nature of the oxides that form on DU and tungsten are quite different. Tungsten does not rapidly oxidize below $600^{\circ} \mathrm{C}\left(1100^{\circ} \mathrm{F}\right)$ in air, or below $500^{\circ} \mathrm{C}\left(950^{\circ} \mathrm{F}\right)$ in oxygen, although a faint discoloration may form (Uhlig 1955). Tungsten is not affected by air at room temperature when it is in other than powder form. Tungsten begins to oxidize at 400 to $500^{\circ} \mathrm{C}(750$ to $930^{\circ} \mathrm{F}$ ) (Fischer 1960). Uranium, however, is a highly reactive metal in oxygen or air. Even at room temperature it tarnishes readily, and at temperatures as low as $125^{\circ} \mathrm{C}\left(250^{\circ} \mathrm{F}\right)$, it forms an oxide at a rate of almost $100 \mu \mathrm{g} / \mathrm{cm}^{2} /$ day (Liebowitz et al. 1961). Ryle et al. note that massive uranium reacts at room temperature with air, oxygen, hydrogen, water, water vapor, and, of course, more reactive substances (Ryle et al. 1960). Tungsten can form at least three oxides: $\mathrm{UO}, \mathrm{UO}_{2}$, and $\mathrm{UO}_{3}$. However, in general, the uranium oxides are not protective. When freshly polished, DU has a dull silvery hue, and when exposed to room air, it becomes a straw color within a few minutes. Within a few days, the film darkens to a blue-black. As the oxide film thickens, it assumes the black powdery appearance of $\mathrm{UO}_{2}$, and the film ceases to be protective. $\mathrm{U}_{3} \mathrm{O}_{8}$ and $\mathrm{UO}_{3}$ are nonprotective from the outset. As uranium-oxide films thicken, they crack and crumble, exposing fresh metal to attack (Fischer 1960). Hence, one would anticipate that under comparable room temperature exposures, there would tend to be more uranium oxide than tungsten oxide present and available.

\subsection{REFERENCES}

ASM International. 1990. Metals Handbook, Tenth Edition, Vol. 2, Metals Park, Ohio, p. 667. Dowding, R. J., et al. 1995. "Tungsten Alloy Properties Relevant to Kinetic Energy Penetrator Performance," Tungsten and Refractory Metals-1994, Metals Powder Industry Federation, Princeton, N.J., p. 3.

Fischer, R. B. 1960. "Tungsten," in Reactor Handbook, Vol. I, ed. C. R. Tipton, Jr., Interscience Publishers, Inc., N.Y.

Leibowitz, L., et al. 1961. "The Kinetics of Oxidation of Uranium Between $125^{\circ} \mathrm{C}$ and $250^{\circ} \mathrm{C}$," J. Electrochem. Soc. 108, 1155-60.

Magness, Lee S. 1993. "Properties and Performance of KE Penetrator Materials," Tungsten and Tungsten Alloys-1992, Metals Powder Industry Federation, Princeton, N.J., p. 15.

Ryle, B. G., et al. 1960. "Uranium," in Reactor Handbook, Vol. I, ed. C. R. Tipton, Jr., Interscience Publishers, Inc., N.Y.

Uhlig, H. H. 1955. The Corrosion Handbook, Wiley and Sons, N.Y. 



\section{SCOPE OF WORK, APPROACH, AND METHODOLOGY}

\subsection{MULTIPHASED APPROACH}

The scope of this report is to assess the environmental mobility of tungsten, its fate in the environment, and its potential toxicity to humans. This study is the foundation of an approach that is multiphased and organized to collect essential data for achieving the goals of a multiyear material life cycle assessment of KEPs. The objective is to isolate and address environmental contributions and health concerns associated with a hierarchy of candidate materials, starting with the base materials (tungsten and DU) and quantifying effects contributed by alloy materials. Throughout the study an advisory board composed of scientific experts and U.S. Department of Defense (DOD) program representatives has helped focus and guide the work. The board's role has been to provide the research team with timely advice on what types of research information would better support decision making on KEP materials.

If equivalent deployment performance of tungsten and DU alloys is achieved, the decision on the material of choice may hinge on the cumulative environmental management and control measures needed for one material vs another in all stages of the material life cycle. The key material life cycle stages for KEPs include manufacturing, transport, storage, testing/training/deployment, and cleanup/recycle. Each stage involves military activities unique to that stage that produce different environmental effects caused by the interplay of several environmental pathways including land, water, air, and the food chain. All pathways are potentially involved in transporting KEP materials.

The approach we take is one that will ultimately feed a computer-based environmental fate and transport model. That is, given a release of tungsten to air, soil, or a water body, how does the tungsten change chemically over time and move within the different environmental media to a point of intake by humans? The input needed for the environmental fate model includes information on how tungsten is changed by chemical and biochemical mechanisms over time; how plant tissue absorbs tungsten from the soil; how animals take tungsten into their tissues, and how the food sources contaminated with tungsten are ingested by humans. Also important are the transport of tungsten to drinking water sources and how tungsten could be suspended or resuspended in the breathing air of a human. Once a human is exposed to tungsten, we need to know how the element was transported within the body. Finally, given an intake of tungsten, what diseases or toxicological effects does it cause? Thus, many parameters must be characterized before an integrated model can be used to evaluate activities in the military life cycle of KEPs and to establish appropriate management/control procedures to minimize risk to humans and other environmental resources.

\subsection{ANALYTICAL STRUCTURE}

The analytical structure of this study has been designed around a science-based assessment of relevant data collected from literature, in contrast to long-term environmental experiments that are outside the scope of this preliminary environmental screening. State-of-the-art mathematical modeling was used to characterize the environmental and biological behavior of tungsten in order to identify critical data 
gaps. Recognition of these lead to a prioritized set of focused experiments and tests to fill in missing information.

\subsubsection{Literature, Industry Interaction, and Model Simulation}

The tungsten literature contains limited information directly useful for understanding tungsten's behavior in the environment. The concept of using tungsten in place of, or in addition to, DU for heavy armor penetrators has been evaluated several times over the past several decades. More recently, several reviews focusing on the toxicity of tungsten have been prepared under Department of Army sponsorship. These reviews focused on several aspects of tungsten in addition to the toxicology of the metal, but none focused solely on tungsten toxicology. In these studies tungsten toxicology data were usually tabulated and were restricted in scope. Few analyses were provided on the potential health impacts of tungsten, the limitations on interpretation of the data, and the specific additional information necessary to better support decision making.

In this study, a broadly based literature search, followed by evaluation and modeling, was aimed at identifying some of the fundamental limitations of available tungsten environmental and health data as well as developing up-to-date information regarding potential environmental impacts of tungsten. To better facilitate a comparison between tungsten and DU, selected information regarding DU was collected.

The literature search involved utilizing relevant data bases, examining the reference sections of acquired articles, and contacting persons with knowledge about tungsten or DU research. Examples of the computer-based systems that were used to obtain information about potentially relevant articles are as follows.

- Applied Science \& Technology Index

- Chemical Abstracts

- DOE 1986-1994, Science and Technology

- DOE 1974-1985, Science and Technology

- Defense Technical Information Center (DTIC)

- Hazardous Substance Data Base

- Medline

- National Aeronautics and Space Administration Aerospace Data Base

- National Technical Information Service (NTIS)

- OASIS, Lockheed Martin Energy Systems (LMES) On-line Library Catalog

- Registry of Toxic Effects of Chemical Substances

- The New York Department of Health library and homepage

- World Wide Web (WWW) including the U.S. Environmental Protection Agency (EPA) WWW server.

Another potential source of tungsten environmental data is private industry. Industries often keep records of personnel for insurance reasons and, while we at Oak Ridge National Laboratory (ORNL) have spent years in some cases performing epidemiological analyses of such records, many difficulties associated with human records were met. The occupational work force within the United States for people exposed only to tungsten metal is so small, and possibly not adequately identifiable, that it did not seem appropriate at this early stage to suggest human-based studies. Later phases of this work will investigate the need for pursuing this longer-term source of data. However, one goal of this study is to. 
maintain efforts to continue to collect relevant data and to catalog studies completed by industries involved in tungsten use.

In collecting data, knowledge about the ultimate use of chemical and biological data dictated the types and forms of data needed. Careful screening of available data was undertaken, and selected data that are consistent with that collected from long-term epidemiological studies for hazardous materials were used to project the toxicity of tungsten. It was not adequate to say that toxicological data were needed. What is actually needed is the development of a model of a disease or toxic condition caused by some type of tungsten intake. Thus, this phase of work required the investigators to critically evaluate data and develop models of potential harm. These models are not detailed computer programs but rather conceptual frameworks around which to weave available data; the focus might be on environmental mobility, plant and animal uptake, or potential potency for biological effects such as cancer, reproductive effects, or neurological effects. Obviously a model for a toxic response for which there are no supporting data could not be developed. The question was, for what types of models were there adequate data to be successful?

Using the approach just described, preliminary comparisons of tungsten and DU have been assembled and data gaps have been identified. The preliminary comparisons presented in this report will be refined in subsequent phases of this program with data collected from narrowly focused tests and experiments.

\subsubsection{Preliminary Material Environmental Comparisons and Key Environmental Concerns}

To make preliminary comparisons of the two base materials, the question central to the issue was "Is there any difference in environmental impacts between tungsten and DU?" Initial meetings with the program's advisory board led to the understanding that, when DOD said environmental effects, the interest was primarily in environmental transport to and effects on humans. Health impacts resulting from injuries were not considered. Of significant and growing importance is the fact that practice ranges and other test sites are becoming more visible with regard to potential radiation exposures from uranium deposited in the soil.

To evaluate whether similar concerns may arise with tungsten, a critical step is to conduct a screening level analysis of the environmental impacts of tungsten penetrators. Building on recommendations of previous studies, the most important unknowns in the critical path were (1) biokinetic distribution within the human body of tungsten after initial exposure and (2) toxic effects of tungsten retained by the human receptor. If these two elements in the critical path remain unknown or uncertain, other types of information cannot be used to solve the central question. These facts must be known early so that additional specific studies can be recommended. Other parts of the overall environmental transport model are also important, and some effort is devoted to identifying environmental mobility factors for tungsten and DU.

Public concerns about new materials introduced into the environment are usually embodied in such questions as:

- Is the new material carcinogenic to humans?

- Is the new material responsible for adverse reproductive or developmental effects?

- Are there any adverse neurological consequences from exposure to the new material?

- Are there any adverse metabolic effects consequent to exposure to the new material? 
The answers to these questions and many others related to the life cycle of tungsten penetrators can be developed only within the context of a phased program of multiyear dimensions. This program begins with a review and interpretation of available data, augmented with simulation modeling, and followed up by specific gathering of data by experiments and their analyses.

\subsection{PHASE I: CY 1995}

Within Phase I of this multiyear program, we concentrated on the issues most central to answering the previous four questions related to potential health impacts. The two most important unknowns in the critical path were (1) distribution of tungsten within the human body after an initial exposure and (2) the toxic effects of tungsten. In addition, because of the importance of environmental transport in delivering environmentally deposited materials to the receptors, our initial effort also includes a review of available environmental transport factors for tungsten. Finally, in an attempt to address a realistic situation, we focused our application efforts on a simplified scenario involving the inhalation and ingestion pathways. The three building blocks, environmental pathways, biokinetics, and potency methods are described briefly in the following sections. In the chapters that follow, each area is developed in detail and integrated into the scenario analyses presented in Chapter 7.

\subsubsection{Environmental Pathways}

Human exposure to tungsten or other penetrator materials requires the bioavailability of the material, which in turn depends on a source term and the movement of the material through the environment. Tungsten may be available for human exposure through the following environmental pathways: air, water, soil, and the food chain. Transfer factors for biological uptake of tungsten and DU used in the exposure model are presented in the environmental transport chapter (Chapter 4). Also presented briefly are the types of environmental transport models that could be used to evaluate tungsten mobility. Although this report describes only briefly several potential exposure pathways, later phases of this program will assess realistic scenarios based on actual experience and projected situations involving water, air, and food chain distribution of tungsten.

\subsubsection{Biokinetics}

Once an understanding of the environmental pathways is established and the environmental transfer factors identified, the next building block for the scenario analysis is the distribution pattern within the human body. Previous work by the authors demonstrated that state-of-the-art modeling efforts based on physiological considerations can be used to increase our understanding of the biokinetic behavior of contaminants in humans. During the first phase, we have found it necessary to formulate a completely new, physiologically meaningful model (e.g., a systemic biokinetics model for tungsten), modifying existing submodels where appropriate (the respiratory tract module), and using existing submodels without modification where appropriate (gastrointestinal tract module). Because of the inadequate treatment in the literature, pursuing a state-of-the-art simulation of the behavior of alternative penetrator materials in the environment and in the human body is the only technically sound approach to identifying and addressing the host of issues that might have an influence on the human health outcome and subsequently the materials life cycle decision process. In this study, the synthesis of fragmented and disparate literature information is guided by the application of biochemical and physiological principles and state-of-the-art assessment tools. 


\subsubsection{Potency Evaluations}

Determination of the relative environmental hazards of tungsten and DU requires a careful understanding of the toxicological aspects of tungsten and DU. Roughly 40 years of biological studies have been devoted to understanding uranium bioeffects, and still there are some uncertainties.

Relatively few data exist for tungsten, and no comprehensive assessment of the tungsten literature has been performed with a focus on quantifying potential human health impacts. This study includes the first serious attempt to evaluate existing literature in terms of quantitative values for the biological endpoints of interest for the environmental perspective (e.g., cancer, reproduction, neurotoxicity, and metabolic effects).

For the toxicological analysis a methodology is applied that allows the use of less-than-optimal data to predict results that would be found with long-term whole-animal cancer studies. It is based on an understanding of the developmental processes in cancer, on relative comparisons, and on the entire knowledge base of well-studied carcinogens. The relative potency methodology that is applied to tungsten has been used to assist the priority ranking of sites within the Installation Restoration Program of the U.S. Air Force and the entire DOD within the Defense Priority Model. The methodology has undergone validity checks through predictions of future outcomes of the National Toxicology Program's two-year animal cancer studies and has been tested and evaluated in 20 open literature publications.

In the following three chapters, these methodological elements are described in more detail and the preliminary findings are documented for use in the scenario analysis. The environmental transport of tungsten and DU is addressed in Chapter 4. 



\section{ENVIRONMENTAL TRANSPORT OF TUNGSTEN AND DU}

\subsection{ENVIRONMENTAL EXPOSURE CONCEPTUAL MODELS}

The manufacture and use of metal penetrators can lead to metal accumulation in the environment, transport through the environment, and eventual human exposure. The potential environmental transport pathways for human exposure to metal penetrators are presented in Fig. 4.1. Test range operation is illustrated, but other material management life cycle stages such as manufacturing and deployment are also potentially major sources of releases into the environment.

Once the penetrator material has reached an environmental medium (i.e., air, soil, or water), and has been chemically or biochemically transformed from the metallic state to a soluble metal compound, the material can be either taken directly into the body (i.e., inhalation, ingestion of soil, and ingestion of water) or introduced indirectly. Indirect routes of exposure could result from the metal being transferred to plants or animals that humans eventually consume. Another potential indirect exposure route is through infant ingestion of its mother's milk. This pathway is not considered further because the transfer of tungsten from mother to milk is more an issue of biokinetics (and is also beyond the scope of this Phase I report). Therefore, the potential indirect exposure routes discussed include ingestion of vegetation, meat (e.g., beef), milk, eggs, and fish. To determine the amount of material that an individual may be exposed to, the source terms, transport to the receptor, and any transfers between environmental media (e.g., soil to plant uptake) are needed. Details about these transport mechanisms and transfer coefficients for air, water/soil, and foodchain pathways are presented in Section 4.3.

Human inhalation or ingestion exposure models can calculate a certain mass intake of tungsten through the pathways just described. The intake depends on the concentration in the exposed medium (e.g., air) to which an individual is exposed, the intake rate, and the exposure time. This model is represented by the following generic equation:

$$
M_{i}=C_{i} * I R_{i} * E T
$$

where

$M_{i}=$ the mass intake of tungsten (or other penetrator material) for exposure pathway $i(\mathrm{mg})$,

$C_{i}=$ the concentration in exposure medium $i\left(\mathrm{mg} / \mathrm{m}^{3}\right.$ in air; $\mathrm{mg} / \mathrm{kg}$ in soil, vegetation, meat, eggs, and fish; or $\mathrm{mg} / \mathrm{L}$ in water and milk),

$I R_{i}=$ the intake rate of exposure medium $i\left(\mathrm{~m}^{3} / \mathrm{y}\right.$ of air; $\mathrm{kg} / \mathrm{y}$ of soil, vegetation, meat, eggs, and fish; or L/y of water and milk),

$E T=$ the exposure time $(\mathrm{y})$.

Equation 4.1 results in a mass $(M)$ of tungsten taken into the body. For use in biokinetic modeling and toxicological evaluations, intake into the body is often expressed as a mass of material per unit mass of body weight. Therefore, the unit intake is calculated as the $M$ in Equation 4.1 divided by the mass of the exposed individual: 


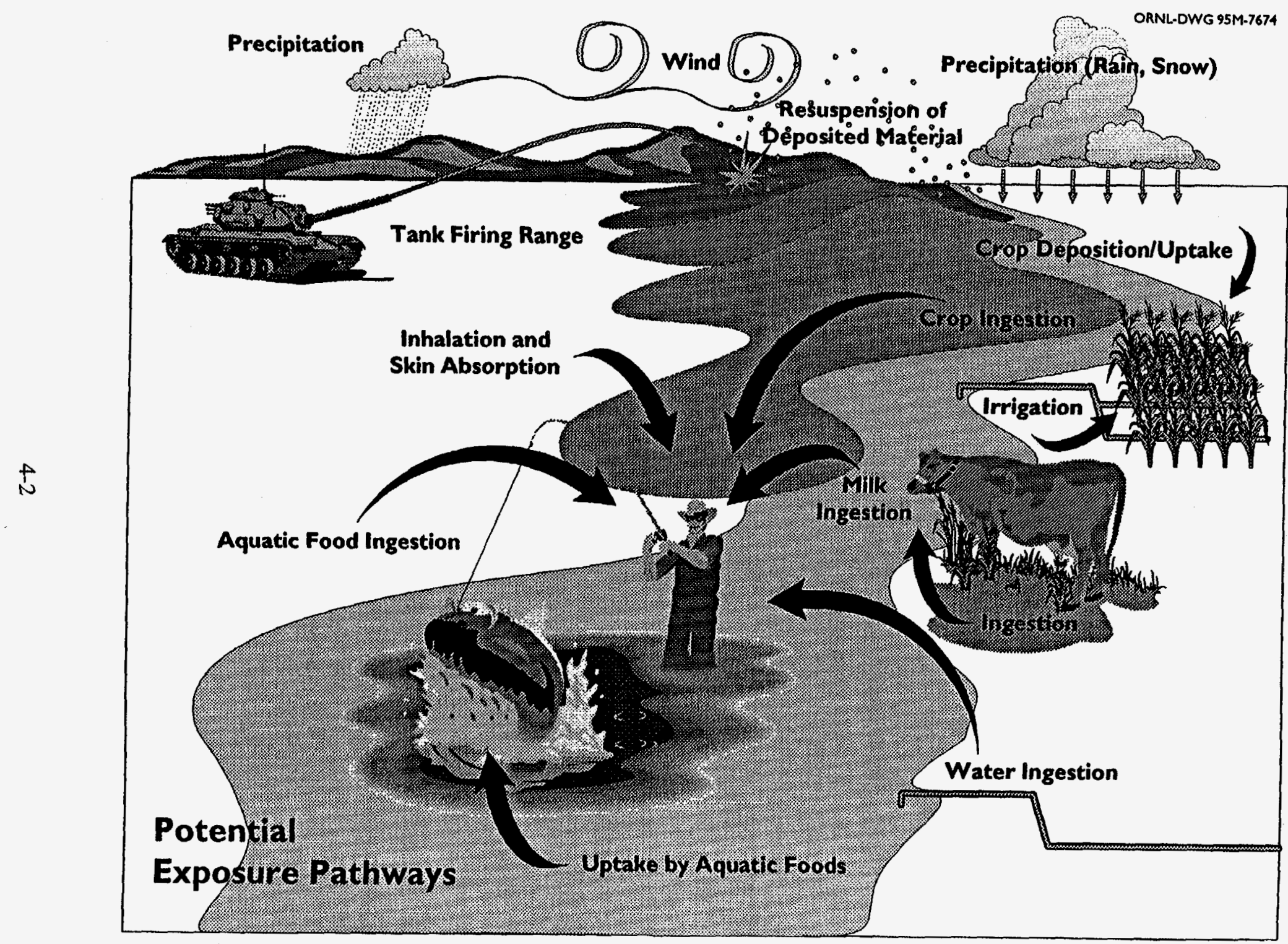

Fig. 4.1. Pictorial representation of environmental pathways. 


$$
I=M / B W,
$$

where

$I=$ the unit intake of tungsten (or other penetrator material) per mass of exposed individual ( $\mathrm{mg} / \mathrm{kg}$ ),

$M=$ the mass intake of tungsten (or other penetrator material) $(\mathrm{mg}$ ),

$B W=$ the body weight of the exposed individual $(\mathrm{kg})$.

The intake rates of various exposure media (e.g., air, water, and vegetation) have been reported in the literature and are presented in Table 4.1. Although there may be a large range for different populations or individuals, using average values is adequate for the type of qualitative, comparative analysis in this report, which is intended to compare tungsten to DU. As long as the same values are used for both evaluations, the absolute numbers are not of critical importance.

The concentrations $\left(C_{i}\right)$ in Equation 4.1 depend on the transport and transfer phenomena for each exposure medium. Computer-based environmental fate and transport models can be used to determine the concentrations needed for Equation 4.1. Section 4.3 ("Environmental Transport and Transfers") describes conceptual transport models for such computer-based models and presents transfer coefficients required to calculate exposures.

\subsection{NATURALLY OCCURRING TUNGSTEN}

Vinogradov et al. (1958) found tungsten in various rock to vary between 0.77 and $2.4 \mathrm{ppm}$. Fu and Tabatabai (1988) reported tungsten to range between 0-2 ppm (dry weight) in an agricultural soil from Iowa and found means of $1.9 \mathrm{ppm}$ and $21.4 \mathrm{ppm}$ in two agricultural soils from New Zealand. The mean value for Iowa soil was $0.89 \mathrm{ppm}$. Furr et al. (1976) found tungsten at $2.1 \mathrm{ppm}$ in soil near Ithaca, New York. In a separate study, Furr and colleagues (1978) found tungsten at $1.3 \mathrm{ppm}$ in soil from the same area. Shaclelette et al. (1978) reported tungsten to range from 5 to $100 \mathrm{ppm}$ (dry weight) in Rocky Mountain trees and shrubs. Furr et al. (1977) found cabbages grown in fly ash-amended soil to accumulated tungsten concentrations from 0.2 to $1.7 \mathrm{ppm}$ dry weight. Levels of tungsten in air, water, and aquatic ecosystems were not found in this initial review.

\subsection{ENVIRONMENTAL TRANSPORT AND TRANSFERS}

Transport through the air, water, soil, and foodchain are described in this section. Nonsite-specific parameters for assessing the environmental transport and transfer of tungsten and DU are also provided. 
Table 4.1. Intake and consumption rates of exposure media

\begin{tabular}{lll}
\hline Exposure medium & \multicolumn{1}{c}{ Value } & \multicolumn{1}{c}{ Source } \\
\hline air & $8400 \mathrm{~m}^{3} / \mathrm{y}^{a}$ & ICRP 1994, ICRP 1975 \\
soil & $0.037 \mathrm{~kg} / \mathrm{y}^{b}$ & EPA 1990, NRC 1975 \\
water & $730 \mathrm{~L} / \mathrm{y}$ & EPA 1990 \\
vegetation & $190 \mathrm{~kg} / \mathrm{y}$ & NRC 1977 \\
meat & $83 \mathrm{~kg} / \mathrm{y}$ & ICRP 1975 \\
milk & $110 \mathrm{~L} / \mathrm{y}$ & NRC 1977 \\
eggs & $17 \mathrm{~kg} / \mathrm{y}$ & ICRP 1975 \\
fish & $8 \mathrm{~kg} / \mathrm{y}$ & ICRP 1975 \\
\hline
\end{tabular}

${ }^{a}$ The inhalation rate is for an average adult, combining inhalation during sleep, rest, and light activity over a year (ICRP 1994).

${ }^{b}$ The soil ingestion rate is for an average person above 7 years old consuming the daily average 365 days of the year and accounts for ingestion of outdoor soils and indoor dust. Children under 7 years old consume double that amount.

'Includes fruit, vegetables, and grain (NRC 1977).

\subsubsection{Air Pathway}

Tungsten can be emitted into the atmosphere from manufacturing the material, from aerosolization of the material upon contact, and from resuspension of material that has deposited onto the soil. Once tungsten is in the atmosphere, humans in the immediate vicinity can inhale the surrounding air, or the tungsten can be dispersed through the atmosphere and transported to a human receptor at a remote location. Fig. 4.2 highlights the inhalation-specific transport pathways.

The first step in quantifying an exposure to airborne tungsten is determining the source term. In the case of manufacturing, tungsten particles may be released in stack emissions. Releases to air from firing of the penetrators (from test ranges or actual military operation) would result from the aerosolization of the tungsten upon contact with the target or other interceptor or from smoke plumes of resulting fires. This source term can be modeled to obtain a concentration in the air.

Once released to the atmosphere, tungsten would transport, disperse, and deposit downwind of the release. Manufacturing emissions resulting in a continuous plume are often modeled using the Gaussian plume equation, which predicts downwind atmospheric concentrations. Releases associated with penetrators contacting a target would be virtually instantaneous. The Gaussian puff equation is often used to estimate downwind concentrations of instantaneous releases. The concentration of tungsten in the air, to which a receptor is exposed, also depends on the amount that is deposited on the soil surface and then resuspended by the wind. Modeling these mechanisms requires knowledge of site-specific parameters (e.g., wind speed and precipitation), precluding further discussion in this generic pathway description. 


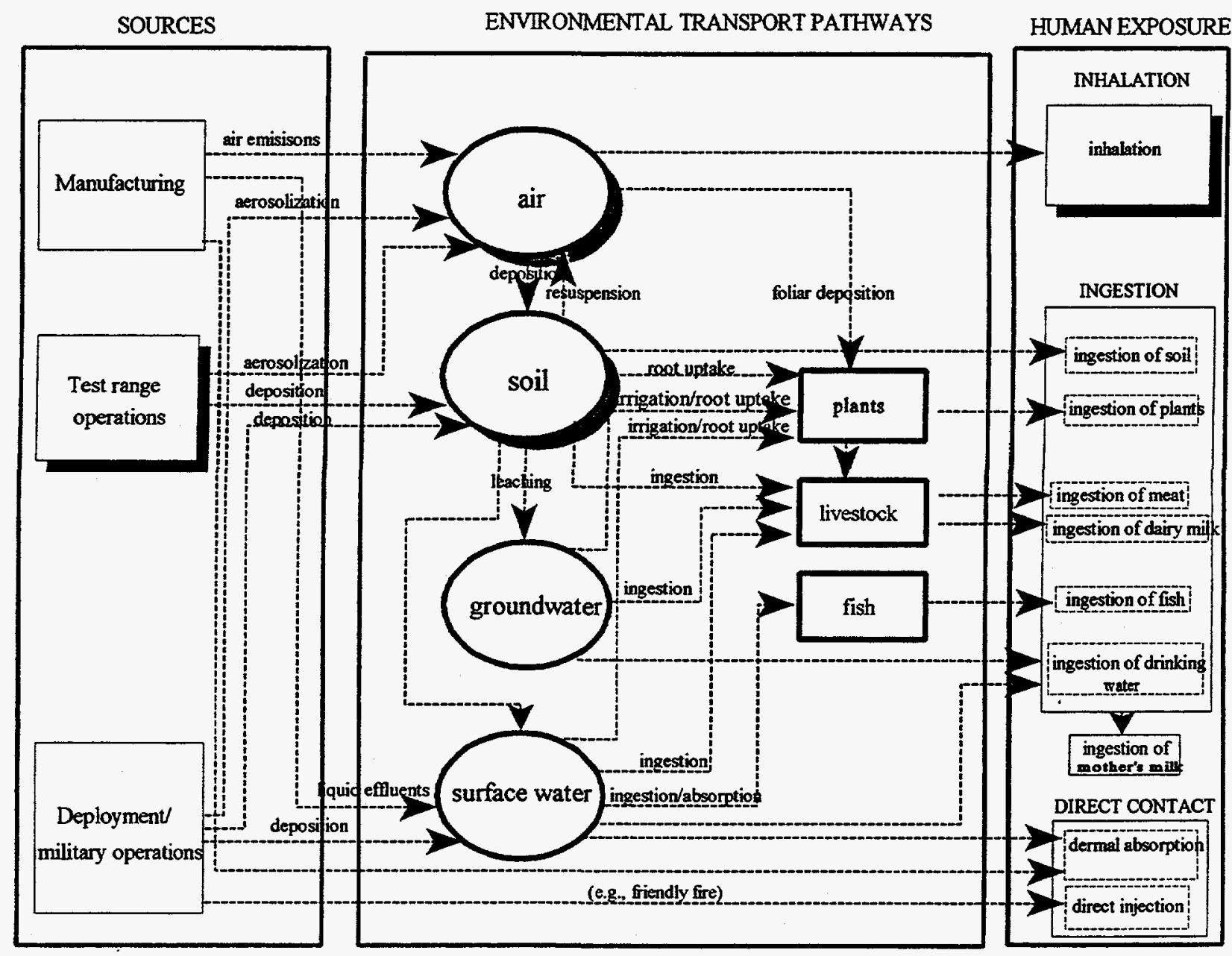

Fig. 4.2. Detailed schematic of environmental pathways.

Using a concentration calculated with the appropriate dispersion model, the mass intake $(M)$ from inhalation is calculated in Equation 4.1. The unit intake ( $I$ ) of tungsten in Equation 4.2 is then used in biokinetic modeling and toxicological evaluations. Which chemical forms of the tungsten become airborne is an important quality to know. Different chemical forms can result in orders of magnitude differences in movement through the environment or human receptors.

\subsubsection{Water and Soil Pathways}

The presence of tungsten or other penetrator material in surface water could result from liquid effluents released during the manufacturing process, from test firings directly into surface waters, and from wet or dry deposition of the metal in the air. In addition, direct deposition of the metal onto soil (in addition to wet or dry deposition from air) could place the metal in the soil. This metal is initially largely insoluble. Dissolution and corrosion will result in chemical compounds of tungsten that could then leach into groundwater or surface water. Human exposure could result from the ingestion of water 
or soil. The actual exposure depends on the temporal and spacial relationship between the contaminated medium and the human receptor. For example, a child could consume contaminated soil long after the tungsten is deposited. With time, some of the metal may leach out of the soil, thus reducing the exposure. In another example, surface water or groundwater might be contaminated but the specific location where the water is contaminated not be used as a drinking water source.

Therefore, transport to the drinking water source would need to be modeled. To conduct a water/soil pathways analysis, a generic conceptual model that incorporates site-specific geometry and water-flow pathways can be developed.

The transport of tungsten in soils (e.g., bioaccumulation in plants and leaching into the ground surface and groundwater) is related to its ability to sorb onto soil particles or solubilize in water. A measure of tungsten's expected behavior in the soil is provided by the soil-water partition coefficient, $\mathrm{K}_{\mathrm{d}}$, which is given by:

$$
\mathrm{K}_{\mathrm{d}}=[\mathrm{C}]_{\text {soir }} /[\mathrm{C}]_{\text {water }} \text {, }
$$

where

$[\mathrm{C}]_{\text {soil }}=$ the concentration of tungsten in the soil $(\mathrm{mg} / \mathrm{g})$ and

$[C]_{\text {water }}=$ the concentration of tungsten in the soil water $(\mathrm{mg} / \mathrm{mL})$.

$\mathrm{K}_{\mathrm{d}}$ is a "lumped" parameter that is essentially the ratio of the concentration of the chemical in all soil components measured in a given study to its concentration in soil water (or extractant or leachate).

No measurements of tungsten $K_{d}$ were identified in this review. However, Baes et al. (1984) found an inverse relationship between an element's $K_{d}$ and soil-to-plant concentration factors (CFs) and used their analysis of tungsten soil-to-plant CFs to estimate a tungsten $K_{d}$ of $150 \mathrm{~mL} / \mathrm{g}$. Using this relationship and a new tungsten CF determined in this review (see following section), a tungsten $\mathrm{K}_{\mathrm{d}}=30$ is predicted.

Using the $\mathrm{K}_{\mathrm{d}}$ and site-specific data in a water and soil transport model, concentrations of tungsten in soil and water can be calculated. These concentrations are then applied to Equations 4.1 and 4.2 to calculate the intake of tungsten.

\subsubsection{Food Chain Pathway}

Assessing human exposure through the ingestion of contaminated foodstuffs requires knowledge about the transfer of tungsten from the environment to the food. Two factors representing the major transfer pathways are presented: soil-plant CF and terrestrial animal biotransfer factor (BTF) (e.g., animal feed to beef). 


\subsubsection{Soil-Plant Concentration Factor}

The uptake of tungsten into terrestrial plants such as vegetables, herbs, fruits, and forage grasses may occur through root uptake or deposition on leaf, stem, or fruit surfaces from the air (e.g., through resuspension of dusts). The root uptake of tungsten from soil into plants may be estimated by:

$$
[\mathrm{C}]_{\text {plant }}=[\mathrm{C}]_{\text {soil }} * \mathrm{CF}_{\text {plant }}
$$

where

$[C]_{\text {plant }}=$ the concentration of tungsten in the edible part of the plant $(\mathrm{mg} / \mathrm{g})$,

$[\mathrm{C}]_{\text {soil }}=$ the concentration of tungsten in the root zone of the soil (mg/g, usually assumed to be the top $15 \mathrm{~cm}$ of soil),

$\mathrm{CF}_{\text {plant }}=$ the concentration factor for tungsten in the edible portion of the plant (unitless).

It follows from Equation 4.4 that the tungsten CF is simply the ratio of tungsten concentration in the edible plant part to that in soil. Estimates of CF for tungsten (Table 4.2) are derived from the studies where tungsten uptake into plants has been measured experimentally in the laboratory or where associated soils and plants have been sampled in field survey studies.

Once the concentration in the plant is calculated, the value is substituted into $C_{i}$ of Equation 4.1 to obtain the mass intake from the human consumption of contaminated plants. This in turn is substituted into Equation 4.2 to obtain the unit mass intake for further biokinetic and toxicological evaluations.

Three studies were found upon which to derive tungsten CFs. Individual "measurements" of the tungsten CF ranged between 0.07 and 3.8. Based on a review and analysis of these three studies, a CF for tungsten of 0.3 is now recommended. This value is approximately 35 times larger than the $C F$ for uranium reported by Baes et al (1984). The recommended tungsten CF value is also larger than the value (0.045) reported by Baes et. al. (1984) by a factor of about eight and three times larger than the value of 0.1 currently used by the International Atomic Energy Agency (IAEA) in performing dose assessments.

A review and analysis of the basis for the tungsten CF value of 0.3 follows. Table 4.2 provides a summary of the individual studies used in this analysis.

Baes et al. (1984) selected a tungsten CF value of 0.045 for use in a terrestrial transport model used by the EPA in demonstrating compliance with the National Emissions Standards for Hazardous Air Pollutants (NESHAPs) under the 1977 amendments to the Clean Air Act (CAA). Two of the references reviewed here were available in 1984, but the tungsten CF derived from these references was higher than the value of 0.018 previously used in performing dose assessments ( $\mathrm{Ng}$ et al. 1968) and was essentially equal to the CF for molybdenum-a tungsten analog. Baes et al. opted to estimate a CF using an approach that took into account the position of tungsten on the periodic table and CFs derived for elements in corresponding rows and groups. This approach predicted a lower CF $(0.045)$ than that derived from the experimental studies but satisfied expected tungsten behavior compared with molybdenum. Upon further review of these two references and examination of an additional reference, a soil-plant tungsten $C F$ of 0.3 now appears more reasonable. 
Table 4.2. Tungsten CFs derived from a review of the literature

\begin{tabular}{|c|c|c|c|c|}
\hline Soil & Plant & $\mathrm{CF}$ & Comments & Reference \\
\hline 0.89 & 0.18 & 0.20 & $\begin{array}{l}\text { Field study. Mean of } 18 \text { soils, } \\
\text { representing the major cultivated } \\
\text { soils of Iowa. Mean of } 22 \\
\text { agricultural plant samples. Plant and } \\
\text { soil samples are not necessarily } \\
\text { related to each other. Both soil and } \\
\text { plant concentrations on a dry weight } \\
\text { (mg/kg) basis. May include } \\
\text { contribution from resuspended dusts. }\end{array}$ & Fu and Tabatabai 1988 \\
\hline 0.1 & 0.38 & 3.8 & \multirow{3}{*}{$\begin{array}{l}\text { Pot Study. Study measuring }{ }^{185} \mathrm{~W} \text { in } \\
\text { barley grown on three different soils } \\
\text { in pot study. Soil and plant values in } \\
\mathrm{mCi} / \mathrm{g} \text { on a dry weight basis. The CF } \\
\text { of } 3.8 \text { is from a fine sandy soil. } \\
\text { Authors believe form taken up by } \\
\text { plants to be } \mathrm{WO}_{4}{ }^{2-} \text {. }\end{array}$} & \multirow[t]{3}{*}{ Wilson and Cline 1966} \\
\hline 0.1 & 0.08 & 0.8 & & \\
\hline \multicolumn{2}{|c|}{ Geometric mean 0.60} & 0.070 & & \\
\hline 1.3 & 0.3 & 0.23 & \multirow{2}{*}{$\begin{array}{l}\text { Field study. The first value is } \\
\text { clover/fly ash and the second is } \\
\text { clover/soil. All values in } \mathrm{mg} / \mathrm{g} \text {, dry } \\
\text { weight. }\end{array}$} & \multirow[t]{3}{*}{ Furr et al. 1978} \\
\hline $\begin{array}{c}6.4 \\
\text { Geometr }\end{array}$ & $\begin{array}{c}2.5 \\
\operatorname{tean} 0.30\end{array}$ & 0.39 & & \\
\hline \multicolumn{2}{|c|}{$\begin{array}{l}\text { Overall geometric } \\
\text { mean }\end{array}$} & 0.33 & $\begin{array}{l}\text { The geometric mean of the geometric } \\
\text { means calculated for each study. }\end{array}$ & \\
\hline
\end{tabular}

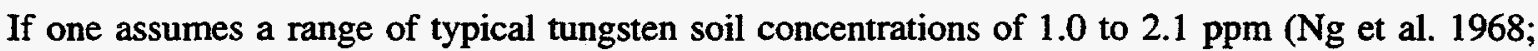
Quinn and Brooks 1972; and Furr et al. 1966), one would predict typical tungsten concentrations in terrestrial plants to vary between 0.3 and $0.6 \mathrm{ppm}$. Quinn and Brooks (1972) reported tungsten in 18 agricultural plants in New Zealand to range between 0 and $0.38 \mathrm{ppm}$. This range overlaps the range predicted from typical soil concentrations using a CF of 0.3 .

\subsubsection{Terrestrial Animal (Livestock) Biotransfer Factor}

The uptake of tungsten by livestock and its accumulation in livestock food products such as meat, milk, pork, eggs, etc., may be estimated by: 


$$
[\mathrm{C}]_{\text {mealmilk }}=[\mathrm{C}]_{\text {feed }} *[\mathrm{Q}]_{\text {feed }} *[\mathrm{BTF}]_{\text {meal milk }} \text {, }
$$

where

$$
\begin{aligned}
& \mathrm{C}_{\text {meat,miik }}=\text { the concentration of tungsten in the food product from livestock (e.g., meat or } \\
& \text { milk) (mg/g), } \\
& \mathrm{C}_{\mathrm{feed}} \quad=\text { the tungsten concentration in animal feed }(\mathrm{mg} / \mathrm{g}) \text {, } \\
& \mathrm{Q}_{\text {feed }} \quad=\text { the amount of feed consumed each day by the animal }(\mathrm{kg} / \mathrm{d}) \text {, } \\
& \mathrm{BTF}_{\text {meatmilk }}=\text { the biotransfer factor for the food product }(\mathrm{d} / \mathrm{kg}) \text {. }
\end{aligned}
$$

This simple model assumes that the concentrations of tungsten in the livestock are from the ingestion of contaminated vegetation only. The concentrations in the livestock calculated using the BTFs are substituted into $C_{i}$ in Equation 4.1 to determine the human mass intake of tungsten. The mass intakes can be summed for each pathway to calculate the total mass intake. Table 4.3 provides a summary of tungsten BTFs.

\subsection{SUMMARY OF AVAILABLE TRANSFER DATA}

Table 4.4 compares the transfer coefficients for tungsten determined in this brief review with those for uranium. The uranium transfer coefficients used for this comparison were obtained as follows:

- Uranium soil-to-plant CF is taken from Baes et al. (1984).

- Uranium milk BTF is taken from $\mathrm{Ng}$ et al. (1977).

- Uranium beef BTF is taken from Baes et al. (1984).

- Uranium egg BTF is taken from $\mathrm{Ng}$ et al. (1988).

- Uranium $\mathrm{K}_{d}$ is taken from Baes et al. (1984).

Table 4.4 indicates that tungsten may be more mobile than uranium in the soil as evidenced by the lower soil-water distribution coefficient, $\mathrm{K}_{\mathrm{d}}$, and the higher soil-to-plant concentration factor, $\mathrm{CF}$.

These measurements have been made for naturally occurring tungsten and uranium, which are, presumably in some chemical form, not metallic. The KEPs, once deposited into the environment, must dissolve and undergo corrosion before becoming mobile in the environment. The relative rates for corrosion of tungsten and DU penetration in the environment depend on the environmental conditions and the particular alloys used. At this time, for anticipated types of alloys, the uranium is expected to have a higher corrosion rate than tungsten for most environmental conditions. If these values are validated by further testing, once tungsten undergoes corrosion it would be expected to present a greater threat to surface water and groundwater contamination than uranium and have a higher potential for uptake into fish; agricultural plants; and grasses, silages, and concentrates used as livestock feeds. However, many tungsten alloys are expected to corrode much slower than DU.

On the other hand, with the possible exception of transfer from feed to beef, tungsten appears to have about the same potential as uranium to transfer from feed to livestock as evidenced by the milk, chicken, and egg BTFs. However measurements of both tungsten and uranium BTF are very few, and there is a large degree of uncertainty regarding these transfer coefficients. 
Table 4.3. Tungsten BTFs derived from the literature

\begin{tabular}{llll}
\hline \multicolumn{1}{c}{ Food } & BTF & \multicolumn{1}{c}{ Comment } & \multicolumn{1}{c}{ Reference } \\
\hline Milk & 0.00021 & $\begin{array}{l}\text { Based on six unpublished } \\
\text { measurements by G. D. } \\
\text { Potter for six fallout sites. }\end{array}$ & $\mathrm{Ng}$ et al. (1977) \\
Beef & 0.037 & $\begin{array}{l}\text { Estimated from stable } \\
\text { tungsten concentrations } \\
\text { measured in unassociated } \\
\text { beef and feed. }\end{array}$ & $\mathrm{Ng}$ et al. (1982) \\
Chicken & 0.3 & $\begin{array}{l}\text { Estimated from BTF bef using } \\
\text { the chicken to beef BTF } \\
\text { ratio for molybdenum } \\
\text { reported by Ng et al. }\end{array}$ & $\mathrm{Ng}$ et al. (1982) \\
& & $\begin{array}{l}\text { Estimated from BTF bef using } \\
\text { the egg to chicken BTF ratio } \\
\text { for molybdenum reported by } \\
\text { Ng et al. }\end{array}$ \\
\hline
\end{tabular}

Although this data is very preliminary, tungsten appears to be more mobile than environmental uranium. Comparing tungsten and DU as base materials for KEPs will require the development of a better understanding of the rate of environmental transformation from the metallic forms to forms available for uptake by plants and animals (CFs and BTFs) and for transport in groundwater. The combination of these two types of data will allow better comparisons between tungsten and DU. 
Table 4.4. Comparison of tungsten and $U$ transfer coefficients

\begin{tabular}{|c|c|c|c|c|}
\hline Coefficient & Tungsten & Uranium & $\begin{array}{c}\text { Ratio } \\
\text { (tungsten/U) }\end{array}$ & Comment \\
\hline Soil-to-plant CF & 0.3 & 0.009 & 33 & $\begin{array}{l}\text { Tungsten CF based on } \\
\text { three studies. Uranium CF } \\
\text { based on two studies. }\end{array}$ \\
\hline Milk BTF & 0.0002 & 0.0002 & 1 & $\begin{array}{l}\text { Tungsten BTF based on six } \\
\text { measurements in one study. } \\
\text { Uranium BTF based on } \\
\text { two studies. }\end{array}$ \\
\hline Beef BTF & 0.04 & 0.0002 & 200 & $\begin{array}{l}\text { No direct measurements of } \\
\text { either tungsten or uranium } \\
\text { BTF. Tungsten estimated } \\
\text { from unassociated beef and } \\
\text { feed. Uranium based on } \\
\text { elemental systematic } \\
\text { assumptions. }\end{array}$ \\
\hline Chicken BTF & 0.3 & 1 & 0.3 & $\begin{array}{l}\text { Tungsten BTF estimated } \\
\text { based on beef BTF and } \\
\text { elemental systematics. } \\
\text { Uranium BTF based on } \\
\text { one study. }\end{array}$ \\
\hline Egg BTF & 3 & 1 & 3 & $\begin{array}{l}\text { Tungsten BTF estimated } \\
\text { based on beef BTF and } \\
\text { elemental systematics. } \\
\text { Uranium BTF measured in } \\
\text { one study. }\end{array}$ \\
\hline Soil distribution $\mathrm{K}_{d}$ & 30 & 450 & 0.07 & $\begin{array}{l}\text { Tungsten } K_{d} \text { estimated } \\
\text { from soil-to-plant } C F \text {. } \\
\text { Uranium } K_{d} \text { measured in a } \\
\text { single study. }\end{array}$ \\
\hline
\end{tabular}




\subsection{REFERENCES}

Baes, C. F. III, et al. 1984. A Review and Analysis of Parameters for Assessing Transport of Environmentally Released Radionuclides Through Agriculture. ORNL-5786. Oak Ridge National Laboratory.

EPA (U.S. Environmental Protection Agency) 1989. Exposure Factors Handbook, EPA/600/8-89/043, Office of Health and Environmental Assessment, Washington, D.C.

Fu, M. H., and Tahatahai, M. A. 1988. "Tungsten Content of Soils, Plants, and Sewages Sludges in Iowa." J. Environ. Qual. 17, 147-148.

Furr, A. K., et al. 1976. "Multielement Uptake by Vegetables and Millet Grown in Pots on Fly AshAmended Soil." J. Agric. Food Chem. 24(4), 885-888.

Furr, A. K., et al. 1977. "National Survey of Elements and Radioactivity in Fly Ashes-Absorption of Elements by Cabbage Grown in Fly Ash-Amended Soil Mixtures." Environ. Sci. Technol. 11(13), 1194-1201.

Furr, A. K., et al. 1978. "Elemental Content of Tissues and Excreta of Lambs, Goats, and Kids Fed White Sweet Clover Growing on Fly Ash." J. Agric. Food Chem. 26(4), 847-851.

ICRP (International Commission on Radiation Protection) 1994. Human Respiratory Tract Model for Radiological Protection, Annals of the ICRP, ICRP Publication 66. Pergamon, Tarrytown, NY.

ICRP (International Commission on Radiation Protection) 1975. International Commission on Radiological Protection, Task Group Report on Reference Man, ICRP Publication No. 23, Pergamon Press, New York.

Ng, Y. C., et al. 1968. "Prediction of the Maximum Dosage to Man from the Fallout of Nuclear Devices," In Handbook for Estimating the Maximum Internal Dose from Radionuclides Released to the Biosphere, UCRL-50163, Pt. IV, Lawrence Radiation Laboratory.

Ng, Y. C., et al. 1977. Transfer Coefficients for the Predictions of Dose to Man Via the Forage-Cow-Milk Pathway from Radionuclides Released to the Biosphere, UCRL-51939, Lawrence Livermore Laboratory.

Ng, Y. C., Colsher, C. S., and Thompson, S. E. 1982. Transfer Coefficients for Assessing the Dose from Radionuclides in Meat and Eggs Final Report, NUREG/CR-2876 UCID-19464, Lawrence Livermore National Laboratory.

NRC (U.S. Nuclear Regulatory Commission) 1977. Regulatory Guide 1.109. Calculation of Annual Doses to Man from Routine Releases of Reactor Effluents for the Purpose of Evaluating Compliance with 10 CFR Part 50, Appendix 1.

Quinn, B. F., and Brooks, R. R. 1972. "The Rapid Determination of Tungsten in Soils, Stream Sediments, Rocks, and Vegetation." Anal. Chim. Acta 58, 299-309. 
Shacklette, H. T., et al. 1978. "Trace Elements in Plant Foodstuffs," In Dehme, F. W. (ed.) Toxicity of Heavy Metals in the Environment Part 1, Marcel Dekker, Inc., New York, pp. 25-68.

Vinogradov, A. P., Vainshtein, E. E., and Pavlenko, L. I. 1958. "Tungsten and Molybdenum in Igneous Rocks (as related to the geochemistry of tungsten)," Geochemistry 5, 497-509.

Wilson, D. O., and Cline, J. F. 1966. "Removal of Plutonium-239, Tungsten-185, and Lead-210 from Soils," Nature 209, 941-942. 



\section{FATE OF TUNGSTEN IN HUMANS}

An assessment of potential health impacts to humans must include a consideration of the routes by which humans are exposed to tungsten and DU in the environment. Intake, either by ingestion or by inhalation, is the principle route of exposure; however, following such intakes it is necessary that the material cross at least one epithelial layer before it has truly gained access to the interior of the body. It is this physiological process and the various factors that control this biological transfer that preclude the accurate assessment of toxicity based simply on intake or administered dose in laboratory studies. While the magnitude of the intake depends on the concentration of tungsten or DU in the environmental pathway and the extent to which humans make use of that pathway, only a fraction of the intake will be transported across the epithelial layers of the respiratory tract or gastrointestinal (GI) tract and gain access to the interior of the body. Once tungsten or DU has entered the blood it is systemic, and it will be distributed among the various organs and tissues of the body and may enter into specific metabolic processes that might enable it to exhibit a toxic action.

The purpose of this section is to model the concentrations of tungsten in the tissues of the body relative to an amount inhaled or ingested and to identify the key processes and variables (physicalchemical, physiological, or metabolic) that control the resultant tissue levels. The approach used to quantify the behavior of tungsten in the gastrointestinal and respiratory tract and within the systemic regions embodies substantial physiological information as well as information specific to tungsten.

At the conclusion of this chapter, we present the results of using the tungsten and uranium biokinetic models to estimate the body tissue content over time of each substance resulting from the lifetime ingestion or inhalation of a unit mass of each element. The results suggest that tungsten much more readily enters body tissues by either main route of environmental exposure, inhalation or ingestion. It is estimated that tungsten is absorbed 25 times more readily by ingestion than DU and 4 times more readily by inhalation than DU. Tungsten is also excreted at a faster rate than DU, but this appears to be offset by the differences in absorption.

\subsection{GASTROINTESTINAL TRACT}

The model of the GI tract used in this report was developed by Eve (1956) and has been used by the International Committee on Radiological Protection (ICRP) for several years (ICRP 1979 1995). The model, shown in Fig. 5.1, divides the GI tract into four segments: stomach (St), small intestine (SI), upper large intestine (ULI), and lower large intestine (LLI). Each segment of the tract is viewed as a compartment whose contents clears into its successor compartment as illustrated in Fig. 5.1.

With the exception of a few chemicals, absorption can be considered to take place in the small intestine. The magnitude of the absorption is simply the fraction of the ingested material that enters the blood stream and is referred to here as the $f_{1}$ parameter. The numerical values of $f_{1}$ are bounded by zero, representing no absorption, and by one for total absorption of the ingested material. In practice, values typically lie between $10^{-5}$ and 1 as shown in Table 5.1 . 


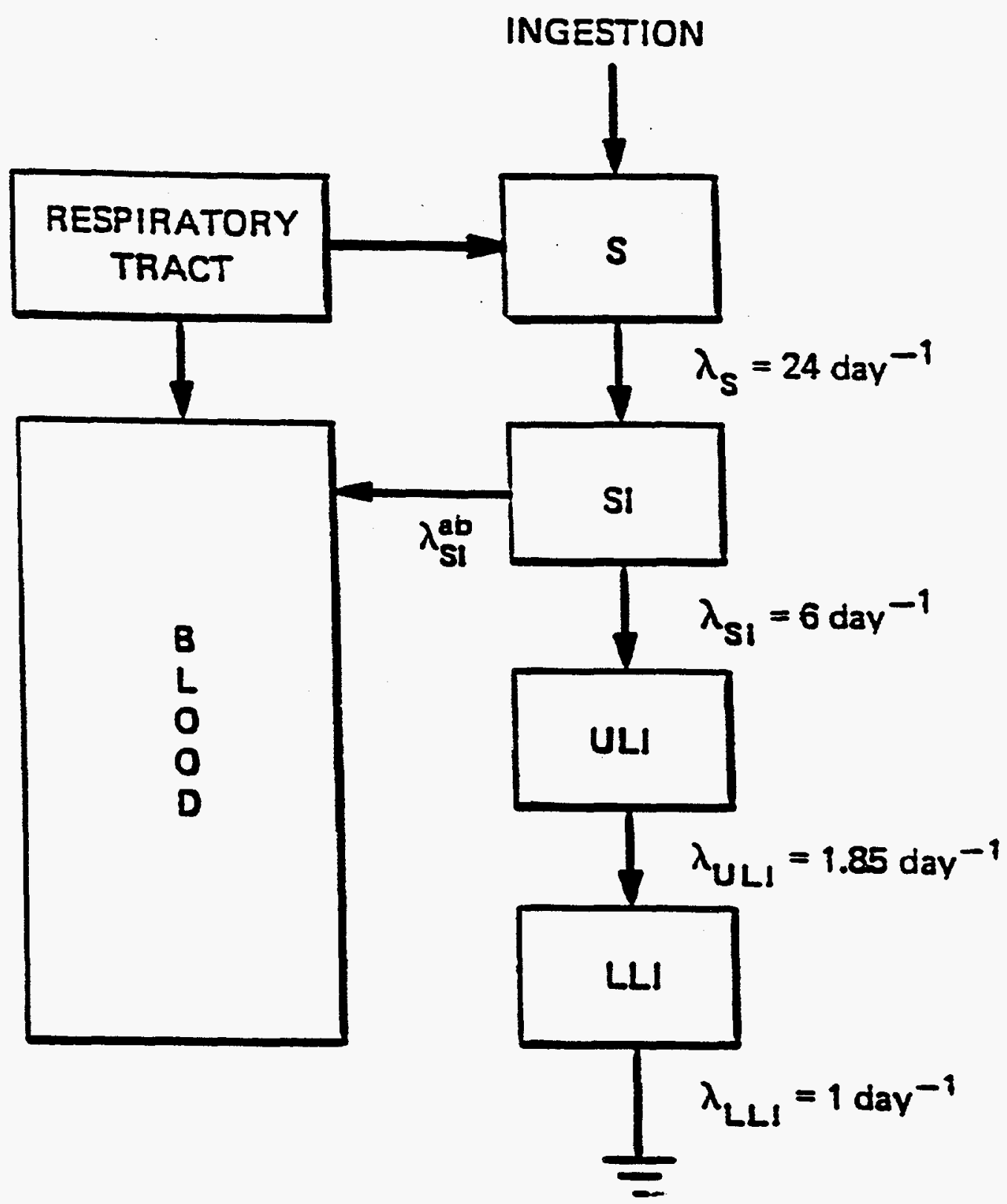

Fig. 5.1. Diagram of the GI tract model (ICRP 1979). Materials enter the GI tract either through ingestion or the swallowing of material cleared from the respiratory tract. Absorption typically occurs from the small intestine, the fraction of the intake that is absorbed is specified by the $f_{\mathrm{L}}$ parameter. The coefficients $\lambda$ denote the fractional rate of transfer of material between the compartments. The transfer coefficient from the small intestine to blood (absorption) $\lambda_{S I}^{a b}$ is given as $\lambda_{S l}^{a b}=\lambda_{S l} f_{1} /\left(1-f_{1}\right)$. 
Table 5.1. Fractional absorption of selected elements

\begin{tabular}{lc}
\hline Element & Value \\
\hline Carbon & 1.0 \\
Potassium & 1.0 \\
Calcium & 0.3 \\
Iron & 0.3 \\
Iodine & 1.0 \\
Zinc & 0.5 \\
Tungsten & 0.5 \\
Uranium & 0.02 \\
\hline
\end{tabular}

The current $f_{1}$ value for tungsten in publications of the ICRP (ICRP 1981) is 0.3 for all compounds of tungsten other than tungstic acid, for which a value of 0.01 is applied. We have reviewed the available experimental information and found the experimental data to range between about 5 to $75 \%$, with absorption depending on the chemical form of tungsten ingested, the nature of the diet, and the age of the subject. Most values are in the range of 30 to $60 \%$. It is suggested that for this study, an $f_{1}$ value of $50 \%$ be considered applicable to the tungsten in the diet (Appendix C). For reference it is noted that the ICRP (1995) recently reviewed the $f_{1}$ data for uranium and used a value of $2 \%$ for dietary uranium. Thus an ingestion intake of 100 units each of tungsten and uranium would result in 50 and 2 units, respectively, being absorbed since tungsten is absorbed 25 times more readily than uranium. Based on this absorption process alone, one cannot rule out the potential for systemic toxicity from ingested tungsten.

\subsubsection{Response to a Unit Intake}

The fractional amount of ingested tungsten present in the segments of the GI tract as a function of time following a unit intake is shown in Fig. 5.2. This figure is based on the model of Fig. 5.1 and uses an $f_{1}$ value of 0.5 for the ingested tungsten; that is, $50 \%$ is absorbed from the small intestine.

\subsection{RESPIRATORY TRACT}

Inhalation is a common route of exposure of humans to potentially hazardous chemicals, particularly in the workplace. An adult human inhales about 20 to $25 \mathrm{~m}^{3}$ of air daily (Table 4.1), and chemicals attached to particles in the air or in the vapor state have the opportunity to interact with about $70 \mathrm{~m}^{2}$ of respiratory tract surface capable of absorption. 


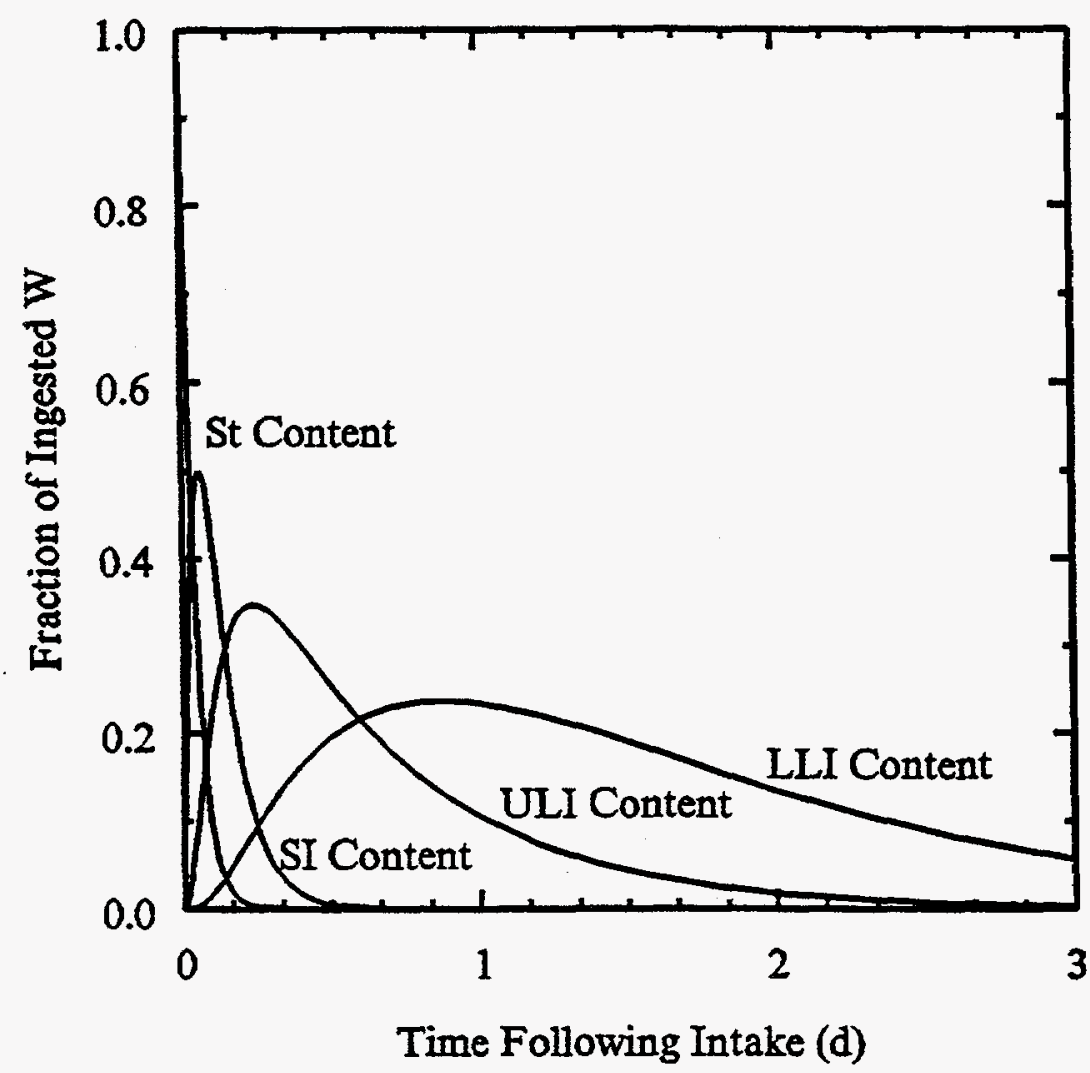

Fig. 5.2. Content of tungsten in various segments (St denotes stomach, SI the small intestine, ULI the upper large intestine, and LLI the lower large intestine) of the GI tract as a function of time after the ingestion of 1 unit. Absorption of tungsten was assumed to take place in the SI as characterized by an $f_{1}$ value

\subsubsection{Respiratory Tract Model}

Recently the ICRP (1994) introduced a new respiratory tract model that includes considerably greater detail and physiological realism than previous models of the respiratory system. Based on the anatomical and morphological considerations and the physical processes by which particles in the inspired air moved through the airways, the ICRP separated the respiratory tract into four major components. These components are (1) the extrathoracic region (ET), comprising the nose and mouth, pharynx, and larynx; (2) the bronchial region $(B B)$, consisting of the trachea and the bronchi; (3) the bronchiolar region $(b b)$, consisting of the bronchioles and terminal bronchioles; and (4) the alveolarinterstitial region $(A I)$, comprising the respiratory bronchioles and the alveolar ducts and sacs. The purpose of this model is to determine the amount of inhaled material deposited in the lung and the clearance of this material into the GI tract and its absorption directly from the airways into blood.

\subsubsection{Deposition of Inhaled Particles}

Inhaled particles and vapors deposit in the airways as a result of physical, anatomical, and physiological processes associated with conditioning and cleansing the inspired air. Particles not removed from the inspired air are available for deposition during the exhalation period in the respiratory cycle. For typical aerosols the total deposition (during inhalation and exhalation) ranges from about 30 to $100 \%$. The deposition fractions for aerosols having lognormal particle size distributions, with the geometric standard deviation taken to be a function of the median particle size 
$\left(\sigma_{\mathrm{g}}\right)$, ranges from 1 to 2.5 at median diameters of 0.0006 to $1 \mu \mathrm{m}$ and above. Figure 5.3 shows the fraction of the inhaled mass deposited in the airways of an adult for aerosols with mass median aerodynamic diameters ranging from 0.1 to $100 \mu \mathrm{m}$.

\subsubsection{Clearance}

Clearance of material from the region of deposition occurs through three pathways. Tungsten and DU material deposited in the forward region of the nose ( $E T$, region) is removed by extrinsic means such as nose-blowing. In other regions, processes act in a competitive manner to transport the material into the GI tract, to lymphatic tissues, and into blood. The physical transport processes are assumed to occur at the same rate for all materials. Absorption into blood is, however, specific to various forms of tungsten and DU. All regions absorb at the same rate, except $E T_{l}$, where no absorption occurs.

Figure 5.4 shows pathways of transport of deposited particles in the compartment model of the respiratory tract. The transfer coefficients shown are the reference values in units of $\mathrm{d}^{-1}$ (per day). Literature on human studies show that in the $A I$ region there is a retention of about $80 \%$ at 50 days and $50 \%$ at one year. In vivo and autopsy measurements, following accidental intakes, show that there are some very long retention times in the lungs. This is represented by three $A I$ compartments, with $30 \%$ in the compartment clearing with a halftime of $30 \mathrm{~d}, 10 \%$ in the very long-term compartment (half-time of $7000 \mathrm{~d}$ ), and the balance in the remaining compartment with a 700-d halftime. The observation from autopsy studies that the concentration in the lymph nodes is about 20 times that in the lungs was used to determine the transfer from $A I$ to $L N_{T H}$.

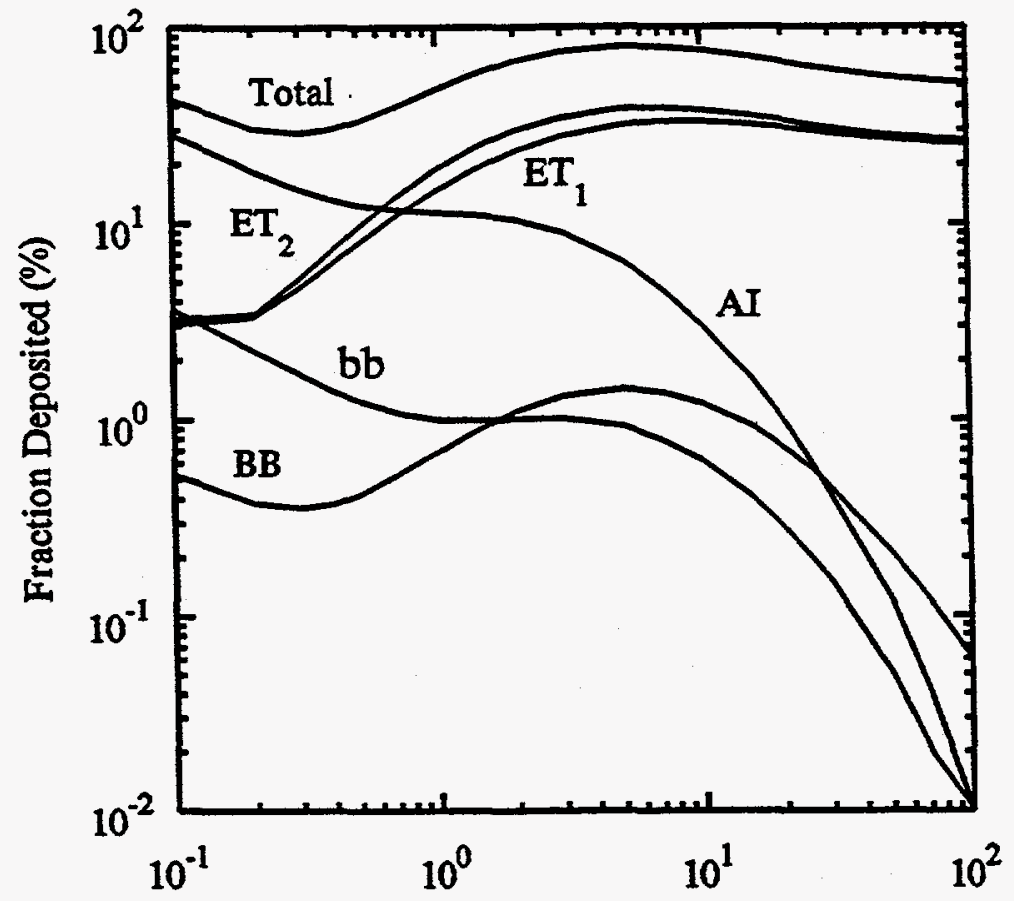

Mass Median Aerodynamic Diamter ( $\mu m)$

Fig. 5.3. Fractional deposition in each region of the respiratory tract for an adult as a function of the mass median aerodynamic diameter (lognormal distribution of particle with a density of $3.0 \mathrm{~g} \mathrm{~cm}^{-3}$ and shape factor of 1.5). 


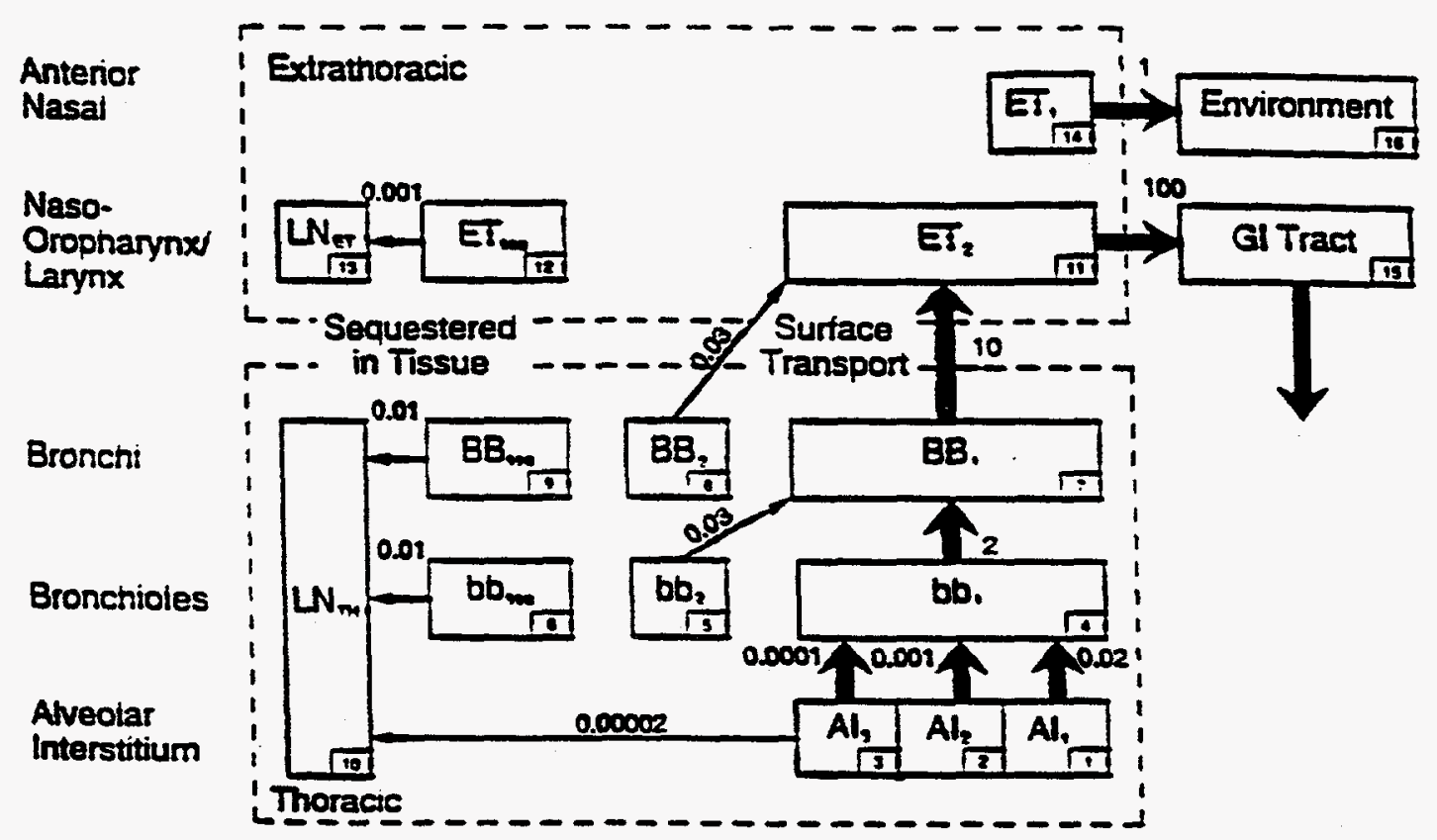

Fig. 5.4. Schematic diagram of movement of deposited particles in the respiratory tract model.

\subsubsection{Absorption}

Ideally, the absorption rate of various forms of tungsten and DU should be determined from a study of the material itself. Some default classifications for absorption are based on the relative rate of absorption: Type $\mathrm{F}$ (relatively fast), Type $\mathrm{M}$ (moderately), and Type $\mathrm{S}$ (slowly). The default absorption types are specified in terms of the $f_{r} s_{r}$ and $s_{s}$ in Table 5.2. The absorption rates, expressed as approximate halftimes, and their magnitude are as follows:

- Type F: $100 \%$ absorbed by 10 minutes. There is a rapid absorption of almost all material deposited in $B B, b b$, and $A I$ and $50 \%$ of the material deposited in $E T_{2}$.

- Type M: $10 \%$ absorbed at 10 minutes, $90 \%$ at $140 \mathrm{~d}$. There is rapid absorption of about $10 \%$ of the deposit in $B B$ and $b b$ and $5 \%$ of material deposited in $E T_{2}$. About $70 \%$ of the deposit in $A I$ eventually reaches blood.

Table 5.2. Parameters of the default absorption types

\begin{tabular}{cccc}
\hline & \multicolumn{3}{c}{ Absorption type } \\
\cline { 2 - 4 } Parameter & $\mathrm{F}$ & $\mathrm{M}$ & $\mathrm{S}$ \\
\hline Fraction dissolved rapidly, $f_{r}$ & 1.0 & 0.1 & 0.001 \\
Rapid dissolution rate, $S_{r}, d^{-1}$ & 100 & 100 & 100 \\
Slow dissolution rate, $S_{s}, d^{-1}$ & - & 0.005 & 0.001 \\
\hline
\end{tabular}


- Type S: $0.1 \%$ at 10 minutes, $99.9 \%$ at 7000 d. There is little absorption from $E T, B B$, or $b b$, and about $20 \%$ of the deposited in $A I$ eventually reaches blood.

\subsubsection{Response Per Unit Intake}

In this study, the principal chemical form of tungsten modeled in the environment is the oxide. The default absorption type applicable to the oxide is Type $\mathrm{M}$. We have further assumed that the aerosol is characterized by a mass median aerodynamic diameter of $5 \mu \mathrm{m}$. The form of DU oxide modeled in the environment is also Type $M$.

As discussed previously, materials deposited in the airways are either absorbed, removed to the GI tract-from which an additional fraction may be absorbed-or removed to lymphatic tissues.

Figure 5.5 shows the cumulative fractions of inhaled tungsten and uranium that are absorbed either directly from the lung or absorbed within the GI tract following movement out of the respiratory tract and being swallowed. As can be seen from the figure, the major absorption route for inhaled tungsten is the GI tract. For DU, the major route of absorption is directly from the lung. Both Type M tungsten and Type M DU in the environment have similar fractional absorptions directly from the lung. For tungsten, about $28 \%$ of the inhaled mass is absorbed; while for uranium, the corresponding value is $7 \%$. Inhaled tungsten is thus readily absorbed, although indirectly from the GI tract.

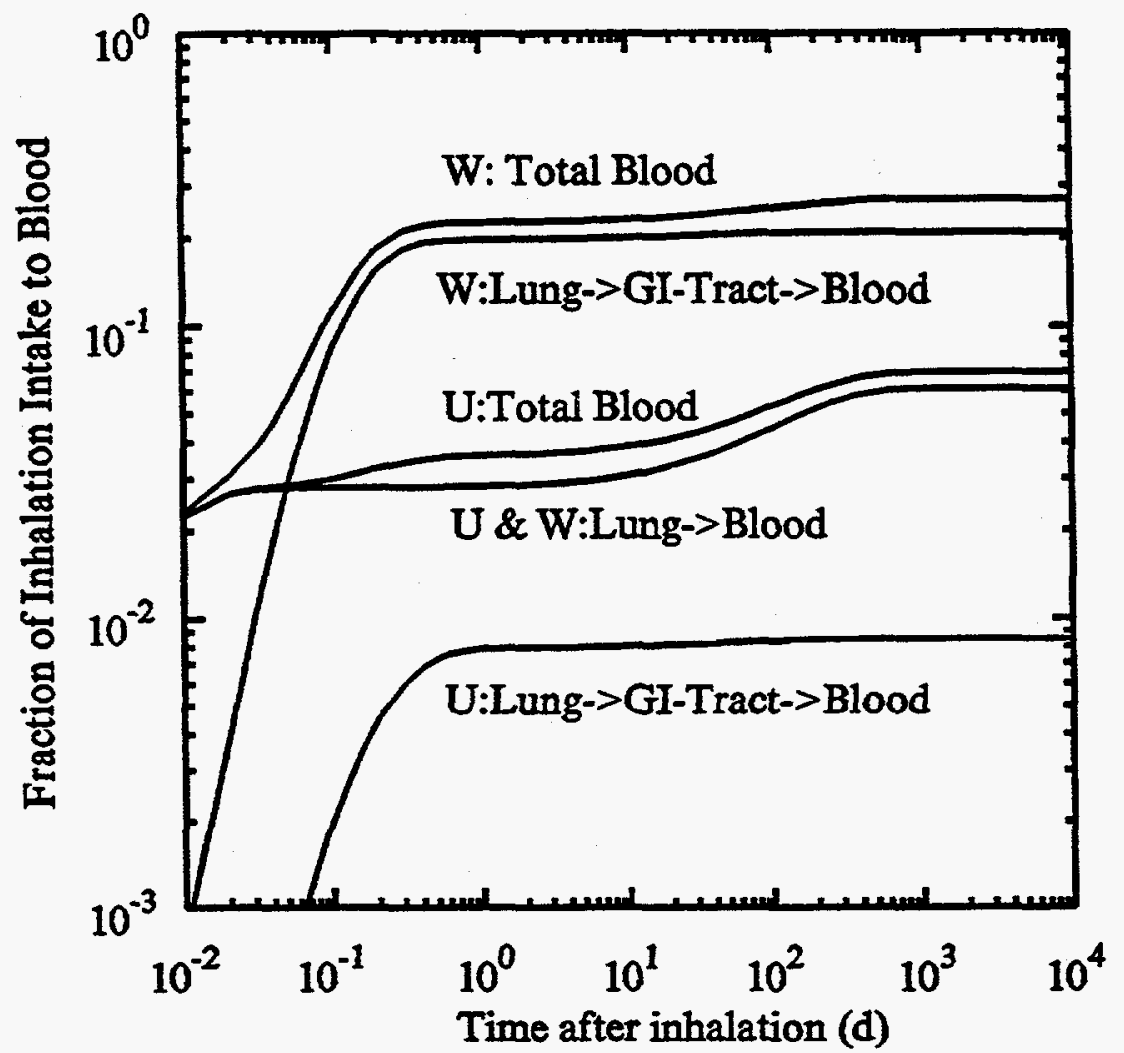

Fig. 5.5. Absorption of tungsten and uranium following an acute intake of Type $M$ material. The absorption directly from the lung and following clearance of the inhaled material into the GI tract are indicated. The inhaled aerosol was assumed to be characterized by a mass median aerodynamic diameter of $5 \mu \mathrm{m}$. 


\subsection{SYSTEMIC TUNGSTEN}

The behavior of tungsten that has been absorbed into the body is extensively reviewed in Appendix C. The available biokinetic model for tungsten used in ICRP Publication 30 (1981) has the limitation that it does not depict biologically realistic paths of movement of tungsten in the body and hence is of limited utility in assessing potential toxicity. For this reason, a new biokinetic model for systemic tungsten with a more realistic structure was developed for this study. The structure is similar to the uranium biokinetic model developed by one of the authors (R.W. Leggett) for the ICRP (1995); see also Leggett (1994). The reader should consult Appendix C for the detailed development of the model.

Because of the lack of information on tungsten absorption in humans, the model developed here is based on experimental data on the biokinetics of radiotungsten in laboratory animals and information on the kinetics of the chemical analog molybdenum or other physiological analogues of tungsten in humans and laboratory animals. The sparse and heterogenous data are unified with a biologically realistic model structure that incorporates information on the physiological processes that appear to control the behavior of heavy metals in the skeleton.

\subsection{TISSUE LEVELS FOLLOWING CHRONIC INTAKE}

The biokinetic model computes the mass of tungsten and uranium in systemic tissues of the body at times following an acute intake or as a consequence of chronic intakes. Environmental exposures typically result in chronic intakes. The mass of tungsten and uranium in the kidney, liver, bone, and other soft tissues of the body as a function of the duration of the chronic exposure are given in Figs. 5.6 through 5.9 for a constant intake rate of $1 \mathrm{~g} / \mathrm{d}$ by inhalation. The figures for ingestion exhibit a similar pattern. The kidney and liver reach equilibrium (no further changes in time) with the intake rate after a relatively short period of time. Equilibrium in the skeleton is not achieved by either element nor by uranium in soft tissue. Table 5.3 summarizes the $\mathrm{W}$ and DU content of systemic organs following a lifetime intake by inhalation and ingestion at a rate of $1 \mathrm{~g} \mathrm{~d}^{-1}$.

\subsection{SUMMARY AND CONCLUSIONS}

This evaluation of the biokinetic behavior of tungsten in the body indicates that a substantial fraction of inhaled or ingested tungsten in the Type $M$ oxide form is absorbed and distributed among systemic tissues of the body. The faster removal rate of tungsten from the systemic regions relative to uranium is offset by the higher absorption from the respiratory and GI tracts. The absorption of tungsten, whether inhaled or ingested, takes place within the small intestine as represented by the $f_{1}$ parameter. In the evaluation presented here, we have used a value of $50 \%$ based on data for various experimental animals which ranged from 5 to $75 \%$. While considerable uncertainties are associated with the biokinetic parameters, it is apparent that tungsten has a greater access to the systemic regions of the body than uranium and that its distribution among these tissues is not negated by its higher excretion rate. The analysis further indicates that with respect to issues of systemic toxicity, inhalation and ingestion of tungsten oxide (Type $M$ ) result in similar fractional absorption of the intakes (28\% and $50 \%$, respectively). Intake of DU oxide (Type M), in contrast, results in much lower absorption both by inhalation and ingestion ( $7 \%$ and $2 \%$, respectively). 


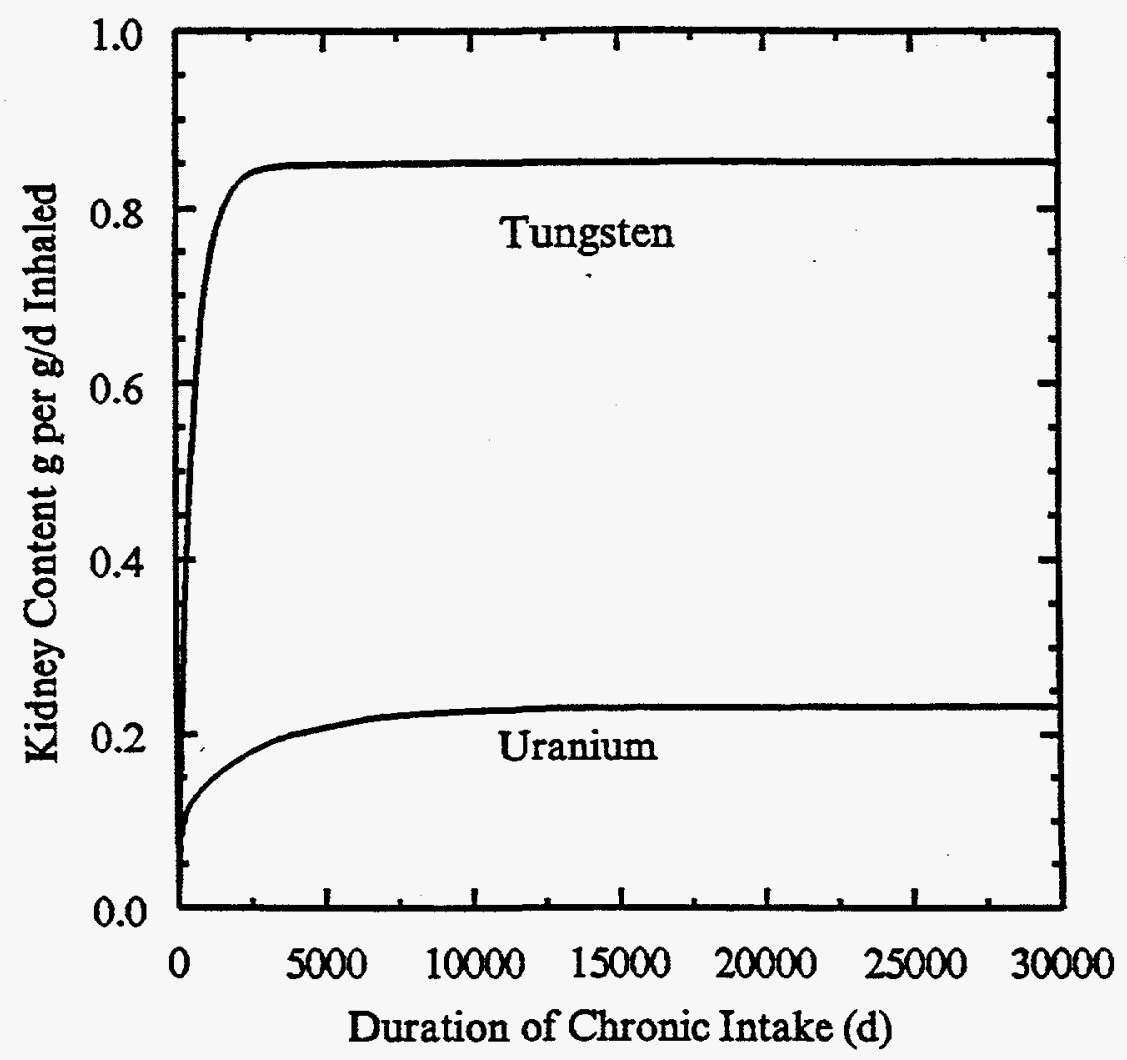

Fig. 5.6. Content of tungsten and uranium in the kidneys of an adult human as a function of the duration of chronic inhalation at a rate of $1 \mathrm{~g} \mathrm{~d}^{-1}$. An aerosol characterized by a mass median aerodynamic diameter of $5 \mu \mathrm{m}$ and absorption Type $M$ was assumed.

The differential biokinetics suggests that, given equal rates of exposure to tungsten and DU, greater amounts of tungsten will accumulate in body tissues. This is a consequence of tungsten's higher absorption through the GI and respiratory tracts. Differential biokinetics is only part of the overall comparison for KEP alternatives. Plant and food uptake factors, which are different for tungsten and DU, as discussed in Chapter 4, are important, but some uncertainty still exists for them. In addition, we know that DU corrodes more rapidly in the environment than tungsten. Thus, the conversion of uranium metal to an environmentally mobile uranium compound occurs more rapidly than for tungsten. In the final analysis of comparative health impacts, this more rapid appearance of environmentally mobile uranium compounds may more than offset the greater uptake characteristic of tungsten identified in this chapter. When additional data are obtained, we will be able to better quantify these relationships through a series of scenario evaluations. 


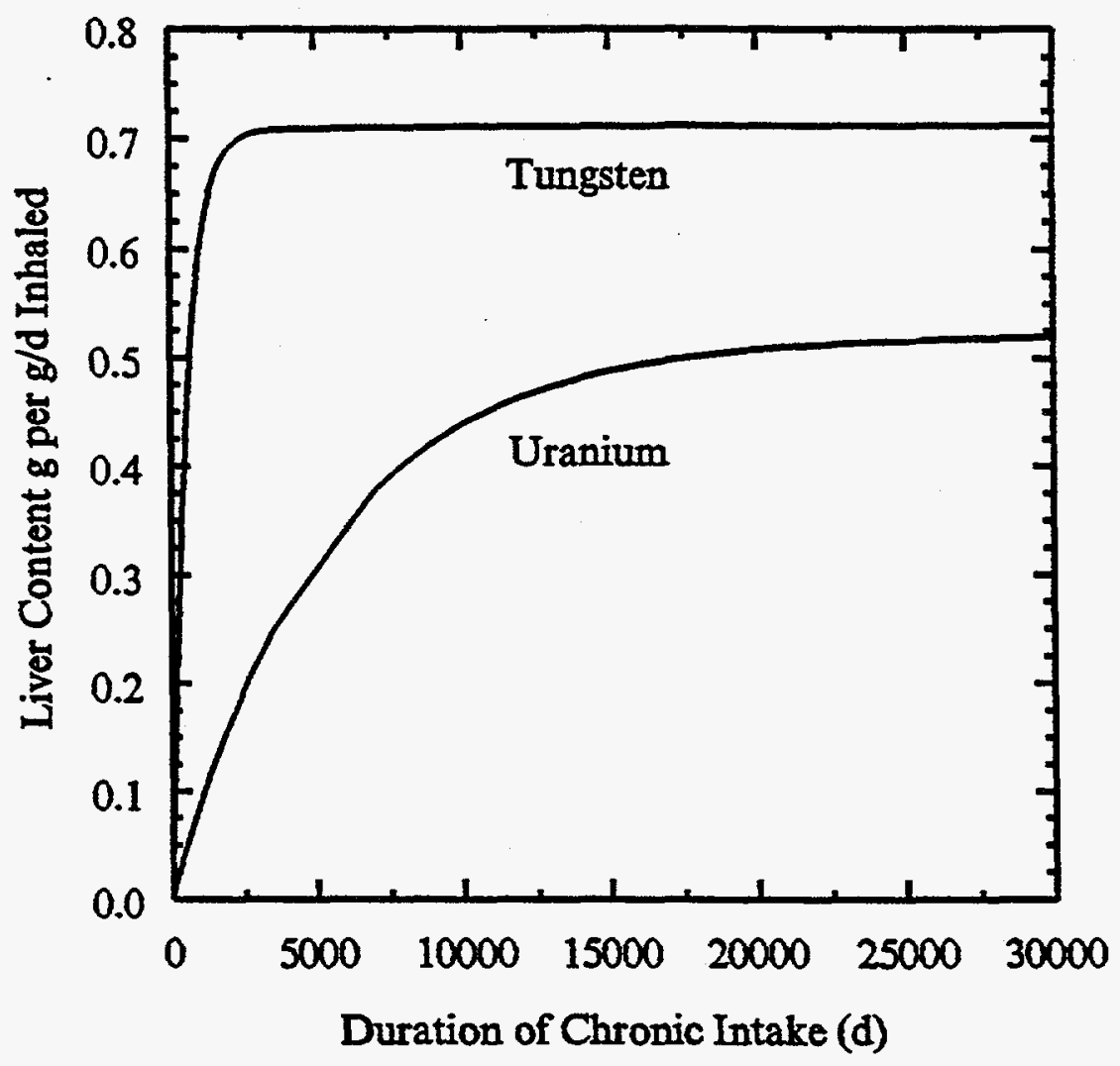

Fig. 5.7. Content of tungsten and uranium in the liver of an adult human as a function of the duration of chronic inhalation at a rate of $1 \mathrm{~g} \mathrm{~d} \mathrm{~d}^{-1}$. An aerosol characterized by a mass median aerodynamic diameter of $5 \mu \mathrm{m}$ and absorption Type $M$ was assumed. 


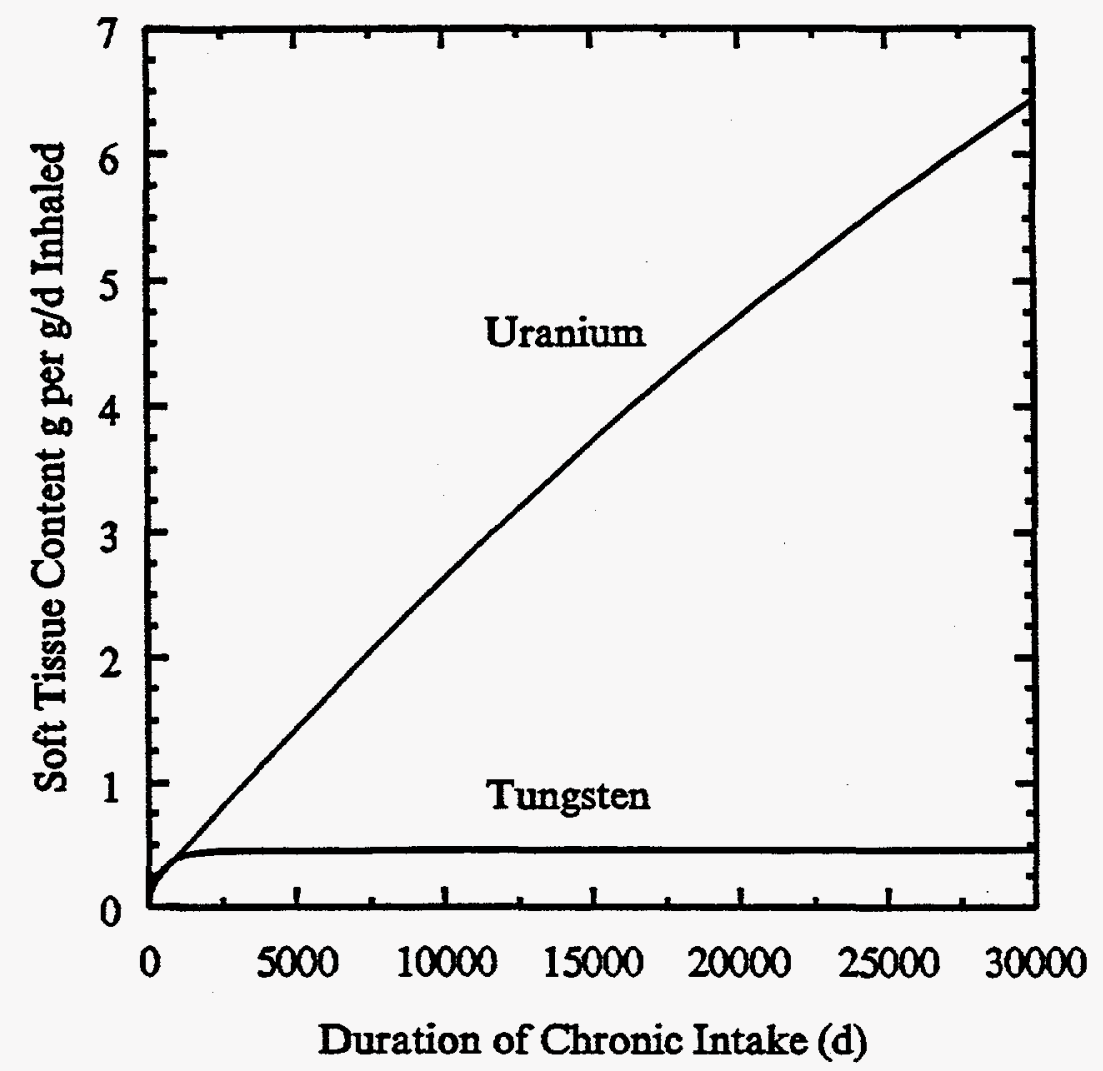

Fig. 5.8. Content of tungsten and uranium in soft tissues other than kidneys and liver of an adult human as a function of the duration of chronic inhalation at a rate of $1 \mathrm{~g} \mathrm{~d}^{-1}$. An aerosol characterized by a mass median aerodynamic diameter of $5 \mu \mathrm{m}$ and absorption Type $M$ was assumed.

Table 5.3. Tungsten and uranium content of body organs (g) assuming chronic intake at a rate of $1 \mathrm{~g} / \mathrm{d}$

\begin{tabular}{lcccc}
\hline & \multicolumn{2}{c}{ Tungsten } & \multicolumn{2}{c}{ Uranium } \\
\cline { 2 - 5 } Organ & Ingestion & Inhalation & Ingestion & Inhalation \\
\hline Skeleton & 30.8 & 17.4 & 4.98 & 17.3 \\
Liver & 1.31 & 0.712 & 0.146 & 0.508 \\
Kidneys & 1.57 & 0.852 & 0.0664 & 0.0232 \\
Spleen & 0.155 & 0.084 & N/A & N/A \\
Other & 1.12 & 0.606 & 1.85 & 6.44 \\
\hline
\end{tabular}




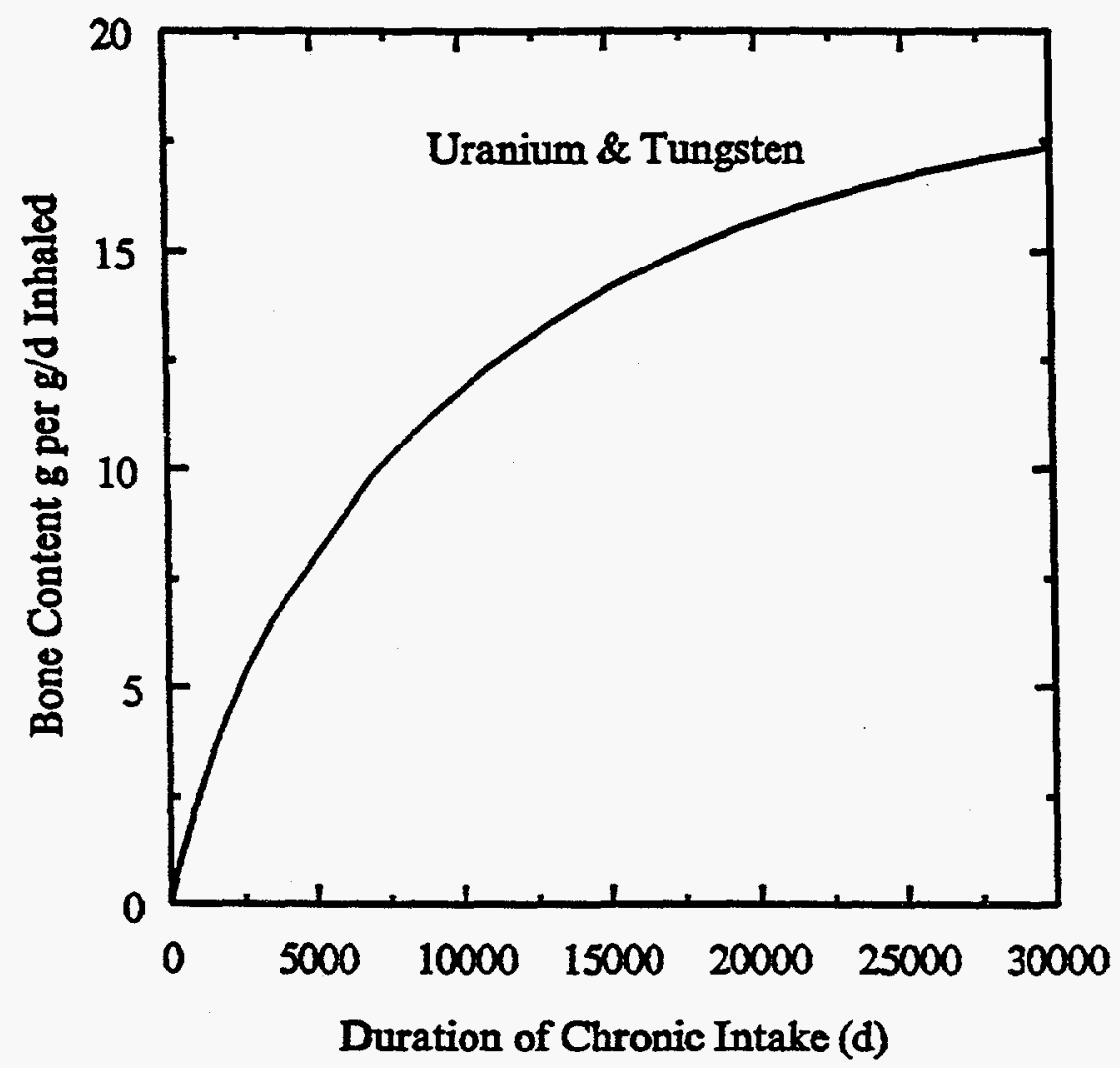

Fig. 5.9. Content of tungsten and uranium in bone of an adult human as a function of the duration of chronic inhalation at a rate of $1 \mathrm{~g} \mathrm{~d}^{-1}$. An aerosol characterized by a mass median aerodynamic diameter of $5 \mu \mathrm{m}$ and absorption Type $M$ was assumed.

\subsection{REFERENCES}

Eve, I. S. 1966. "A Review of the Physiology of the Gastrointestinal Tract in Relation to Radiation Doses from Radioactive Materials," Health Phys. 12, 131-161.

International Commission on Radiological Protection. 1973. Alkaline Earth Metabolism in Adult Man, ICRP Publication 20, Pergamon Press, Oxford.

International Commission on Radiological Protection. 1994. Human Respiratory Tract Model for Radiological Protection, ICRP Publication 66, Pergamon Press, Oxford.

International Commission on Radiological Protection. 1979. Limits for Intakes of Radionuclides by Workers, Publication 30, Part 1, Pergamon Press, Oxford.

International Commission on Radiological Protection. 1981. Limits for Intakes of Radionuclides by Workers, Publication 30, Part 3, Pergamon Press, Oxford.

International Commission on Radiological Protection. 1993. Age-Dependent Doses to Members of the Public from Intake of Radionuclides, Part 2: ICRP Publication 67, Pergamon Press, Oxford. 
International Commission on Radiological Protection. 1995. Age-Dependent Doses to Members of the Public from Intake of Radionuclides, Part 3: ICRP Publication 69, Pergamon Press, Oxford.

Leggett, R. W. 1994. "Basis for the ICRP's Age-Specific Biokinetic Model For Uranium," Health Physics 67, 589-610. 



\section{IMPLICATIONS OF TUNGSTEN TOXICITY FOR HUMANS}

The goal of this chapter is to evaluate the potential of tungsten for producing biological responses in humans from absorption of materials as described by the biokinetics in Chapter 5 . The aim is to characterize biological impacts to humans, develop an understanding of the uncertainties in impacts, and then develop strategies for reducing those uncertainties.

Investigations discussed in this chapter include both noncancer and cancer effects. The noncancer effects evaluated in detail are neurotoxicity, reproductive toxicity, teratogenesis, and metabolic effects. Metabolic effects were found not to pose significant hazards in environmentally relevant scenarios. Pulmonary toxicity has been reviewed previously for "hard metal" forms of tungsten, associated with the production and use of cutting and grinding tools, and is not considered in this document. A summary of studies for the remaining noncancer effects is displayed in Table 6.1.

\subsection{NEUROTOXICITY}

\subsubsection{Quality and Quantity of Data}

The literature, about both humans and animals, is sparse but suggestive of possible neurotoxic effects of tungsten or tungsten compounds; a summary of the neurotoxicity studies is displayed in Table 6.1. Studies of occupationally exposed workers whose exposure included cobalt, a known toxin from hardmetal dusts, found persons with signs and symptoms such as loss of appetite, nausea, and an impaired sense of smell; in one study, some also reported headaches and dizziness (Kaplun and Mezentseva 1959; Vengerskaya and Salikhodzhaev 1962). A small study was done recently on hard-metal workers having pulmonary problems and reporting memory deficits (Jordan et al 1990). Testing demonstrated significant deficits in verbal memory and attention. Unfortunately, these workers had also been exposed to possible confounders, including cobalt, and an unidentified organic solvent. Organic solvents themselves often demonstrate neurotoxicity.

A few animal studies have given indications of tungsten's potential for neurotoxic effects. The two main such studies are in the Russian literature (Nadeenko 1966; Idiatullina 1981) and suffer from a lack of experimental detail included in the written accounts and in one case from certain ambiguities or even conflicts in the details of results and conclusions that are reported. However, taken together, the results that are described are of sufficient substance that they bear serious consideration. The main indicators of neurotoxicity include behavioral changes, cholinesterase (ChE) activity depression, decrements in conditioned learning and reflexes, and structural brain changes (lesions) as described subsequently; more detailed descriptions of the evidence are presented in Appendix F.

\subsubsection{Indicators of Neurotoxicity}

Some of the indications of neurotoxicity in acute animal studies (single high-dose exposures) include uncoordinated movements, trembling, sudden jumps, excitation, reduced motor tone, and even hindlimb paralysis in some animals. Effects have been observed in several mammalian species. Subacute experiments (about 30-d duration) in rats revealed dose-dependent blood $\mathrm{ChE}$ activity, inhibition in response to oral (Nadeenko 1966) or inhalation (Idiatullina 1981) exposure to sodium tungstate. ChE 
Table 6.1. Evidence for tungsten's possible noncancer toxicity

\begin{tabular}{|c|c|c|c|}
\hline Endpoint & No. main studies/species & $\begin{array}{l}\text { Lowest dose or exposure } \\
\text { at which effect observed }\end{array}$ & Indicators \\
\hline \multirow[t]{2}{*}{ Neurotoxicity } & 3/human & $\mathbf{N A}^{\mathbf{a}}$ & $\begin{array}{l}\text { Loss of appetite, impaired } \\
\text { sense of smell, nausea, } \\
\text { headache, dizziness, } \\
\text { memory and attention } \\
\text { deficits }\end{array}$ \\
\hline & $2 /$ rat & $0.005 \mathrm{mg} / \mathrm{kg} \mathrm{bw}$ for $8 \mathrm{mo}$ & $\begin{array}{l}\text { Behavioral changes, } \mathrm{ChE}^{\mathrm{a}} \\
\text { activity decreases, } \\
\text { impaired learning and } \\
\text { reflexes, brain lesions }\end{array}$ \\
\hline \multirow[t]{3}{*}{ Reproductive toxicity } & $3 / \mathrm{rat}$ & $\begin{array}{l}0.005 \mathrm{mg} / \mathrm{kg} \mathrm{bw} \text { for } 8 \mathrm{mo} \\
0.5 \mathrm{mg} / \mathrm{m}^{3} \text { for } 4 \mathrm{mo}\end{array}$ & $\begin{array}{l}\text { Embryotoxicity; fetal } W^{*} \\
\text { accumulation; decreased } \\
\text { sperm motility }\end{array}$ \\
\hline & $2 /$ mouse & $\begin{array}{l}18.4 \mathrm{mg} \mathrm{W/kg} \mathrm{bw;} 120 \mu \mathrm{g} \\
{ }^{185} \mathrm{~W} / \mathrm{kg} \mathrm{bw}\end{array}$ & $\begin{array}{l}\text { Embryotoxicity; fetal and } \\
\text { maternal } W \text { accumulation, } \\
\text { relevant tissues }\end{array}$ \\
\hline & $\begin{array}{l}\text { 4/in vitro chick limb bud; } \\
\text { hen and chick oviduct; rat } \\
\text { ovarian tissue }\end{array}$ & $\begin{array}{l}150 \mu \mathrm{\mu} ; \\
0.2 \mathrm{mM} ; \\
1 \mathrm{mM}\end{array}$ & $\begin{array}{l}\text { Inhibited chondrogenesis; } \\
\text { blocked progesterone } \\
\text { receptors; stimulated } \\
\text { adenyl cyclase activity }\end{array}$ \\
\hline \multirow[t]{3}{*}{ Teratogenicity } & $3 /$ rat & $\begin{array}{l}0.005 \mathrm{mg} / \mathrm{kg} \mathrm{bw} / \mathrm{d} \text { for } \\
8 \mathrm{mo}\end{array}$ & $\begin{array}{l}\text { Decreased skeletal } \\
\text { ossification, defects in } \\
\text { brain, kidney, bladder }\end{array}$ \\
\hline & 1/mouse & $120 \mu g{ }^{135} \mathrm{~W} / \mathrm{kg} \mathrm{bw}$ & $\begin{array}{l}\text { Fetal skeletal W } \\
\text { accumulation }\end{array}$ \\
\hline & $1 /$ in vitro chick limb bud & $150 \mu \mathrm{M}$ & Inhibited chondrogenesis \\
\hline
\end{tabular}

abbreviations: $\mathrm{bw}=$ body weight; $\mathrm{ChE}=$ cholinesterase; $\mathrm{W}=$ tungsten.

is an enzyme necessary for normal nerve transmission in many brain pathways as well as much of the peripheral nervous system. Blood ChE depression is often a marker for depressed brain ChE and may be associated with changes in brain function, resulting in effects on cognition, mood, and attention in humans and animals.

Chronic low-dose oral sodium tungstate exposure has resulted in somewhat depressed blood ChE and in dose-related adverse effects on conditioned learning in rats at doses of 0.05 and $0.5 \mathrm{mg} / \mathrm{kg} / \mathrm{d}(50$ and $500 \mu \mathrm{g} / \mathrm{kg} / \mathrm{d}$ ) for 7 months (Nadeenko 1966.) Biochemical changes in rat brain and structural lesions in the cerebral cortex of rats (and possibly rabbits) were seen at the higher dose levels in this study. Chronic oral exposure for 8 months to $0.005 \mathrm{mg} / \mathrm{kg} / \mathrm{d}$ resulted in effects on reflex activity (Nadeenko and Lenchenko 1977). The results of these long-term, low-dose oral exposures are bolstered by observations reported by Idiatullina (1981) on effects resulting from inhalation exposures of rats, even though the route of exposure is not as relevant to the scenario of concern as the oral route. 
Subchronic (4-month) sodium tungstate inhalation exposure to moderate doses also resulted in modest decreases in blood $\mathrm{ChE}$; the changes showed a step function rather than dose dependency (Idiatullina 1981). Lesions were also seen in the brains of these inhalation-exposed rats.

\subsubsection{Evaluation of Significance}

No studies of tungsten-induced developmental neurotoxicity in animals or humans have been located. If tungsten exposure does indeed decrease $\mathrm{ChE}$ activity as suggested by long-term low-dose rat studies, this suggests effects on the cholinergic pathways of the brain and could result in effects similar to those seen in persons exposed to other $\mathrm{ChE}$ inhibitors such as organophosphate (OP) pesticides and OP nerve agents. Exposure to such substances has been shown to result in difficulty with attention, memory, depression, anxiety, irritability, language use, and other cognitive functions (Savage et al 1988; Metcalf and Holmes 1969; Levin, Rodnitzky, and Mick 1976; Sidell 1992). Links between tungsten exposure and $\mathrm{ChE}$ action in humans have not been observed or scientifically established under any exposure conditions including routine industry exposure, accidents, or administered doses. There is no evidence of how much material would be required to produce a neurotoxic effect in humans or whether such unknown quantities would be encountered as a result of military activity involving KEP use. The evidence for neurotoxic effects is summarized in more detail in Appendix $F$ and has been critically reviewed by Munro, Ambrose, and Watson (1994).

Because of the differential biokinetics between tungsten and DU, equal exposure rates to tungsten and DU will result in greater amounts of tungsten being accumulated in the systemic tissues. However, equal exposure rates would probably not occur in a comparative environmental analysis because uranium may be converted from a less soluble metallic form to a soluble chemical compound more rapidly than tungsten. High resistance to corrosion and low rates of forming soluble tungsten would tend to significantly limit the potential for long-term, low-dose exposure to tungsten. When additional data on conversion rates and transport through the environment is obtained, we will be able to better quantify comparative relationships through a series of scenario evaluations.

\subsection{REPRODUCTIVE TOXICITY}

\subsubsection{Quality and Quantity of Data}

The literature on reproductive effects is relatively sparse, but more studies in vivo and in vitro are available than on neurotoxicity. No data on humans are available. Several Russian reports from the laboratory of Nadeenko and colleagues detail long-term, low-dose rat studies on embryo survival and development and one on tungsten levels in the fetuses. It is not entirely clear how many of the papers give data on different experiments, but several experiments were performed and gave basically consistent results. In addition, a Swedish group (Wide and colleagues) also studied tungsten as well as other metals including molybdenum in mice; their results on embryotoxicity paralleled those of the Russian investigators despite an entirely different experimental design (acute high-dose injection). They also studied tungsten distribution in maternal, placental, and fetal tissue at various times after injection on three different days of gestation. Their results confirm the Russian finding that tungsten crosses the placenta and accumulates in fetal tissue. The results of these investigations are summarized as follows in somewhat more detail; they are presented in full in Appendix F. 


\subsubsection{Indicators of Reproductive Toxicity}

Tungsten has been observed to have in vivo embryotoxic effects in mice exposed acutely (Wide 1984) and rats given chronic peroral exposure (Nadeenko and Lenchenko 1977; Nadeenko et al 1977, 1978). These investigators observed significantly increased death and resorption of embryos, both before and after implantation. Wide, Danielsson, and Dencker (1986) showed that tungsten accumulates in fetal tissue in a distribution study; autoradiographic analysis demonstrated preferential tungsten uptake in fetal neural tissue on day 12 of gestation. These investigators also induced embryotoxic effects in vitro, showing that tungsten inhibited cartilage formation in chick embryo limb bud cells at concentrations equivalent to those causing increased fetal resorption in vivo (Wide, Danielsson, and Dencker 1986).

In vitro studies show that tungstate has the potential to interfere with the pituitary-gonadal hormonal control system via effects on ovarian adenyl cyclase. It may also interfere with progesterone support of the uterine environment for pregnancy maintenance via blocking of progesterone receptors. The distribution study of Wide, Danielsson, and Dencker (1986) revealed accumulation of tungsten in ovarian follicles, pituitary, and placental tissues, as though it might be binding to receptor populations involved with reproductive functions. Long-term exposure of mice to tungsten in drinking water was shown to lower progesterone levels in pregnancy, although quantitative data were not reported (Wide, Danielsson, and Dencker 1986).

Thus, rodent studies and in vitro experiments on enzyme systems and steroid hormone receptors suggest strongly that tungsten has the potential to interfere with reproduction both by exerting direct embryo- or fetotoxic effects and possibly also by disrupting maternal hormonal systems crucial to the normal maintenance of pregnancy. Whether these effects extend to humans is not known.

Furthermore, one animal study suggests potential effects on male fertility. A study by Idiatullina (1981) reported decreased sperm motility in rats (to about $90 \%$ of control value) in response to subchronic inhalation exposure. The decreased motility was accompanied by "weakly expressed hyperemic vessels" observed histologically in the testes, which was more pronounced in the $1.0 \mathrm{mg} / \mathrm{m}^{3}$ exposure group than in the $0.5 \mathrm{mg} / \mathrm{m}^{3}$ group.

\subsubsection{Evaluation of Significance}

Although the number of studies is relatively small, taken together the in vivo and in vitro studies are suggestive of a potential for long-term oral tungsten exposure at low-levels to exert an adverse effect on mammalian reproduction, particularly on the female reproductive system. Neither the Russian nor the Swedish studies give any information on dose-response relationships as only one dose was used in any of their studies with the exception of one in vitro study. The in vitro portion of the Wide, Danielsson, and Denker (1986) study demonstrated dose-related inhibition of chick limb bud cartilage formation by tungstate.

Idiatullina's (1981) observation of tungstate inhibition of sperm motility implies a possible threshold, and the response appears to behave as a step function rather than showing dose-related increments. No effect was seen at $0.1 \mathrm{mg} / \mathrm{m}^{3}$; the responses at 0.5 and $1.0 \mathrm{mg} / \mathrm{m}^{3}$ were 10 and $12 \%$ inhibition or essentially equivalent. 
Reproductive toxicity of the types observed in animal studies have the potential to affect all individuals of reproductive age. At this point, the importance of reproductive toxicity in a comparative assessment for humans cannot be determined. The data presented in this section must be confirmed, and relative rates of conversion from metal to soluble chemical compounds must be determined for DU and tungsten. The more important biological effects of tungsten may be offset by the fact that uranium metal corrodes to an environmentally mobile form more rapidly than tungsten. This would tend to significantly limit the potential for low-dose exposure to soluble tungsten. When additional data are obtained, we will be able to better quantify these relationships through a series of scenario evaluations.

\subsection{TERATOGENESIS}

\subsubsection{Quantity and Quality of Data}

The literature on developmental toxicity is more limited than that for reproductive effects but is suggestive of tungsten exerting teratogenic effects on mammalian development. Although only one group of Russian investigators reported teratogenic effects (Nadeenko and colleagues), they used an environmentally relevant route of exposure (oral) and long-term, low-dose $(0.005 \mathrm{mg} / \mathrm{kg}$ ) administration. The group performed several studies and obtained consistent results; the effects of tungstate administration were compared to the effects of other metals, including molybdenum. The reports omit some key experimental details, including identification of rat strain, but overall the consistency of results is rather persuasive of an effect. A Swedish study in mice (Wide 1984) failed to demonstrate any developmental toxicity although embryotoxicity was observed, as in the Russian work. However, as detailed in the following, experimental and species differences may account for the lack of concordance in results for this endpoint. These studies did not use graded doses, so no information on possible dose-dependence of the effects can be derived from these studies. More complete information is presented in Appendix F.

\subsubsection{Indicators of Teratogenic Effects}

Nadeenko and colleagues report consistently observing inhibiting effects on rat skeletal development as a result of chronic low-dose oral exposure of the mother to tungstate (Nadeenko and Lenchenko 1977; Nadeenko et al 1977, 1978), although Wide (1984) failed to see such an effect in an acute injection experiment in mice. In the mouse experiment, sodium tungstate was injected only on day 3 or day 8 of gestation (out of a 21-day gestation period). In Wide's hands, molybdenum did delay skeletal ossification, so the difference in tungsten's effect may be attributable to species differences or to the differences in duration or timing of exposure with respect to the stage of embryonic development. As documented previously, Wide's group later confirmed the Russian observation that tungsten does cross the placenta and accumulate in the fetus (Wide, Danielsson, and Denker 1986). This study showed a faintly observable late accumulation of labeled tungsten in the fetal skeleton and a preferential early accumulation of tungsten in fetal neural tissue on day 12 of gestation.

The in vitro work of Wide, Danielsson, and Denker (1986) in which they report dose-dependent inhibition of cartilage development in chick limb bud cultures is consistent either with a fetotoxic effect or with the possibility that tungsten may induce the type of developmental abnormality (delayed ossification) described by Nadeenko et al $(1977,1978)$ and Nadeenko and Lenchenko (1977). 
Nadeenko et al. (1978) also observed a few abnormalities of rat fetal brain, kidney, and bladder that they considered were likely a result of their extremely low dose chronic tungstate exposure regimen.

\subsubsection{Evaluation of Significance}

Further work would be highly desirable to replicate the results of Nadeenko and colleagues; in the absence of more data, it can only be said that the existing literature is suggestive of tungsten having the potential to induce developmental abnormalities in mammals. The observation of neurotoxicity in adult animals, brain abnormalities in rat fetuses in chronic experiments, and preferential early uptake of tungsten by fetal neural tissue also raises the critically important issue of developmental neurotoxicity, which has not been evaluated experimentally in any previous work.

At this point the importance of teratogenic effects in a comparative assessment cannot be determined. The data presented in this section must be confirmed, and relative rates of conversion from metal to soluble chemical compounds must be determined for DU and tungsten. The more important biological effects of tungsten may be offset by the fact that uranium metal corrodes to an environmentally mobile form more rapidly than tungsten. When additional data are obtained, we will be able to better quantify these relationships through a series of scenario evaluations.

\subsection{OTHER NONCANCER EFFECTS}

\subsubsection{Immunotoxicity}

Searches were performed, but no studies were found relating to tungsten effects on the immune system except as it has been used to stimulate inflammatory responses in the lung. It appears that no systematic evaluation of potential immunotoxicity is likely to have been performed, particularly for chronic low-dose systemic tungsten exposure. This is an area for which at least preliminary studies, perhaps subacute high-dose experiments, should be considered.

\subsubsection{Toxicity Enhancement}

The toxicity of tungsten to macrophages is strongly enhanced by the presence of cobalt as in hardmetal dusts. The effect is not caused by cobalt alone, as its toxicity is much less (see Appendix F). These findings suggest that if development of tungsten as KEP material is pursued, tungsten toxicity should be tested both alone and in combination with whatever substances are considered as components of alloys. In vitro screening tests should be considered at least as a first stage of evaluation.

\subsection{METHODS FOR ESTIMATING THE CARCINOGENIC POTENTIAL OF TUNGSTEN}

\subsubsection{Why This Biological Endpoint?}

A major consequence of society's cancer concern is that all newly introduced substances must undergo scrutiny. The potential use of tungsten as a substitute for DU in KEPs is no exception. 
However good DU is as a heavy armor penetrator, it does possess a unique characteristic within materials of commerce-it is radioactive. The majority of $\mathrm{DU}$ is ${ }^{238} \mathrm{U}$, and this isotope has a half-life of $4.5 \times 10^{9}$ years. Such a long half-life means that the isotope is nearly stable. Nonetheless, it does undergo radioactive decay emitting various rays and forming radioactive daughter elements.

Concurrent with the radioactive decay comes a potential for causing cancer if the isotope or its decay products are deposited within a human body. This fact drives concern about the need to prevent the spread of depleted uranium over practice ranges, other sites, and over equipment. Thus, the main contrasting point of reference for any potential replacement for DU is whether or not the replacement could cause cancer and how potent the new candidate could be in comparison with DU. The fact that health impacts from DU result primarily from its chemical toxicity on the kidney takes a back seat to the fear of cancer. Appendix D provides a balanced view of uranium exposure regulations and their relationship to the degree of uranium enrichment in ${ }^{235} U$.

A quick review of the available data for tungsten revealed that there are no classic cancer studies that provide a statistically significant conclusion in either direction. The lone study that reported this biological effect was inconclusive. However, the question can be examined less directly. The question that we address in this study is modified from "is tungsten a carcinogen" to "if tungsten is found to be a carcinogen, what would be its potency?" The potency question and the possible answer are highly relevant but different from the cancer question that can be answered only by results from longterm animal studies or epidemiological studies. The description and results of the relative potency projection methodology follow. After that presentation, a practical comparison with DU is made.

\subsubsection{Cancer Potency Based on Relative Potency Considerations}

Starting with the fact that neither epidemiologically based nor laboratory-based cancer studies are available for tungsten, it was necessary to use a relative potency methodology that had proven itself in a variety of other situations. The relative potency approach is a data-intensive, assumption-free approach. An estimate of the relative potency (RP) of hazard " $H$ " compared with a well-studied reference agent "R" can be obtained by comparing the doses $\left(D_{H}\right.$ and $\left.D_{R}\right)$ required to cause the same level of response in a particular biological test model considered to be relevant to the disease of interest. Then an estimate of the relative potency is:

$$
R P=\left(D_{R} / D_{H}\right)
$$

In this study, the sparse body of data on tungsten was assembled and evaluated using five different reference compounds. (Explicit data are presented in Appendix G.) The reference compounds were Benzo(a)pyrene, nicotine, cisplatin, aflatoxin, and cyclophosphamide. The use of a single reference material or a collection of reference agents that were tested in only a few bioassays would not make use of some tungsten data. The use of multiple reference agents ensured that all of the tungsten bioassay data would be evaluated. Figure 6.1 contains plots of tungsten data compared with each of the five reference agents as well as the combined plot for which all comparisons are normalized to the standard reference agent, benzo(a)pyrene, which is defined as having a potency of 1.0 . On the basis of the very sparse data, evidence from the combined figure suggests that the potency of tungsten, if determined to be a carcinogen, would be in the neighborhood of 0.04 relative to benzo(a)pyrene, with one standard deviation going from 0.004 to 0.4 . This calculation of potency is based on a very preliminary analysis of a very sparse data set for tungsten. Consequently, the estimate may not have the same reliability that has been obtained in the evaluation of other substances. It must be stressed that a relative potency analysis does not demonstrate that tungsten is a carcinogen. What has been 


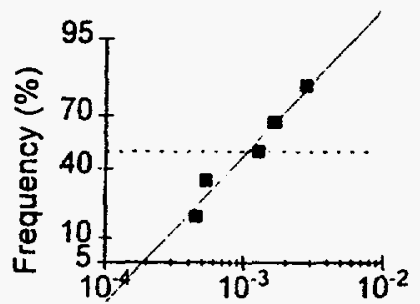

Sodium Tungstate to Aflatoxin B1

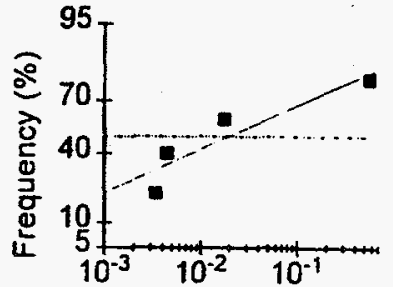

Sodium Tungstate to Cyclophosphamide

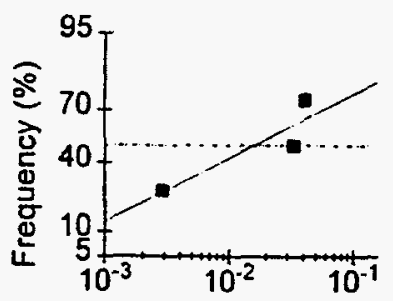

Sodium Tungstate to Cisplatin
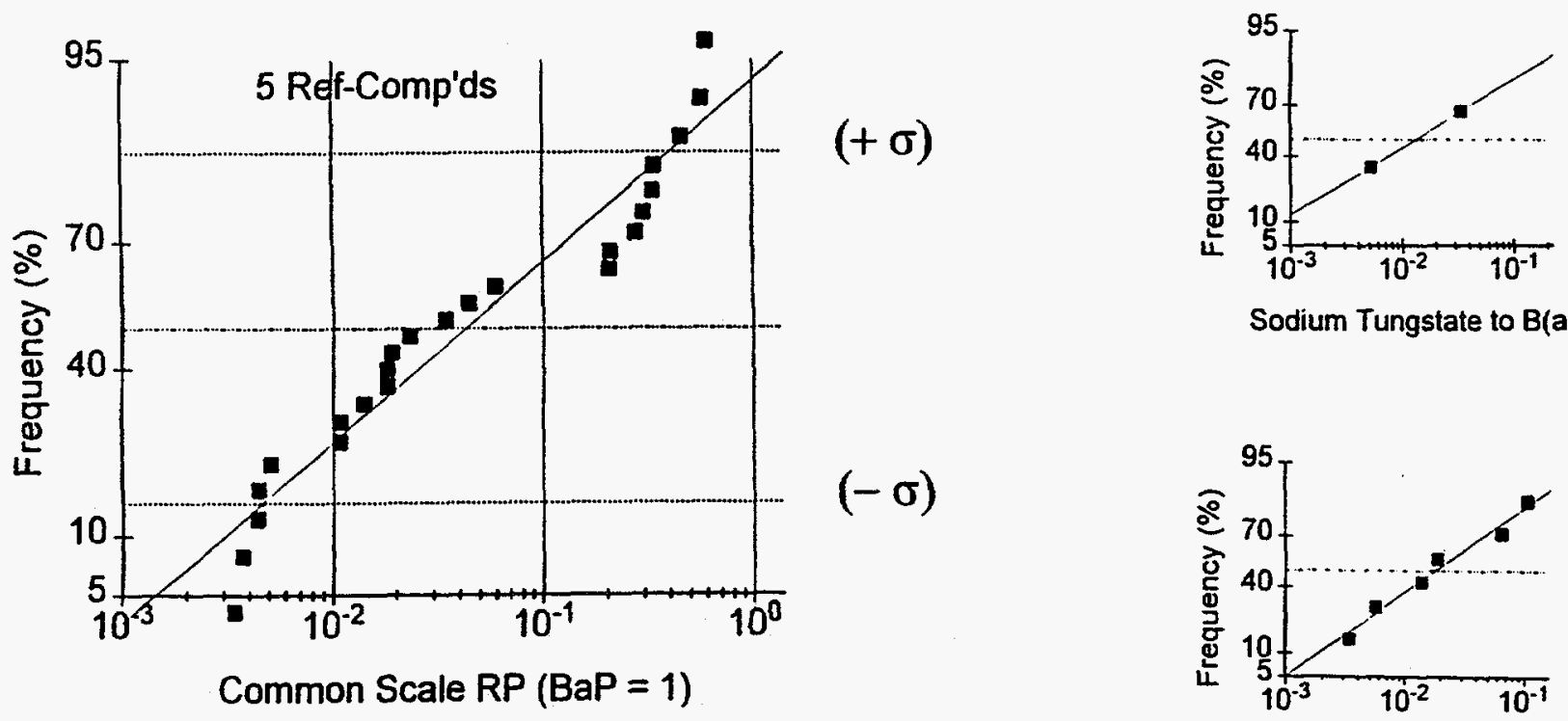

Sodium Tungstate to $\mathrm{B}(\mathrm{a}) \mathrm{P}$

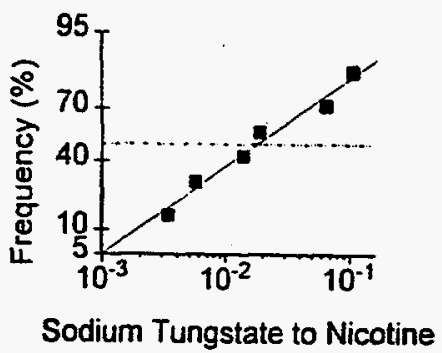

Fig. 6.1. Relative comparisons between tungsten and five reference compounds.

accomplished is that a preliminary value of potency is derived which in previous tests of other substances has been found to be approximately correct when those materials were tested and found to be carcinogens. The potential of tungsten for producing health effects in humans is only speculative at this point.

\subsubsection{Practical Application-Comparison of Tungsten and DU}

With the previous qualifications in mind, the relative potency obtained for tungsten can be related to a larger body of information in toxicology. This capability is one of the major strengths of the relative potency concept since it is designed to build on previous information rather than to require all supporting information to be developed de novo. Within the relative potency methodology, there is 
a way to incorporate the dose-response information developed from human epidemiology studies (Jones, Owen, Trabalka, Barnthouse, and Easterly 1991). Risk coefficients, based on human epidemiology and derived by the EPA Carcinogen Assessment Group (CAG), are used to estimate risk for a compound which might, at some time in the future, become reclassified as a carcinogen. Risk coefficients for beryllium oxide, chromium VI, acrylonitrile, nickel subsulfide, coke oven emissions, nickel refinery dust, arsenic, benzene, cadmium, and benzidine were selected because (1) they reflect the biological response of humans to hazardous agent exposures and (2) they do not seem to include the inflated upper limits found in risk coefficients derived by the EPA based on animal experiments. With slope factors adjusted to account for oral intake and from an analysis of RP and slopes for the 10 substances, an estimate of risk based on $\mathrm{RP}_{\mathrm{i}}$ and dose $\mathrm{D}_{\mathrm{i}}$ of any interviewing chemical becomes

$$
\text { Risk }=4.7 \times R P_{i}(\mathrm{mg} / \mathrm{kg}-\mathrm{d})^{-1} \times D_{i}(\mathrm{mg} / \mathrm{kg}-\mathrm{d})
$$

This risk factor is based on data that are normalized to the primary reference agent, benzo(a)pyrene. Moreover, it is adjusted for a lifetime (70-year) exposure because the risk factor is based on EPA slope factors for the previously listed human carcinogens. Results of the tungsten evaluation are presented in the context of these epidemiologically derived risk factors in Fig. 6.2. If there were an interest in maintaining a lifetime risk from continuous exposure at or below the $1 \times 10^{-4}$ level, the dose rate of tungsten would need to be at or below

$$
\begin{gathered}
\text { Dose }_{\mathrm{w}}=1 \times 10^{-4} /[4.7 \times 0.04]=1.9 \times 10^{-3} \mathrm{mg} /\{\mathrm{kg} \text {-day }\}, \\
\text { Dose }_{\mathrm{w}}=1.9 \mu \mathrm{gg} /\{\mathrm{kg} \text {-day }\} .
\end{gathered}
$$

This might be contrasted with a similar dose from DU. Figure D.4 of Appendix D contains information on the chronic ingestion levels of DU necessary to produce a particular radiation dose as a function of the degree of uranium enrichment in the isotope ${ }^{235} U$. From this figure, it is seen that about $15 \mathrm{~g}$ of ingested DU results in approximately $1 \mathrm{rem}$ of effective dose. Because there is a large knowledge base relating radiation dose and fatal cancer risk, the relationship between the mass of ingested DU and the resulting radiation dose forms the basis for a comparison between the cancer risk from radiological effects of uranium and the potential chemical potency of tungsten to produce cancer.

From ICRP 60 (1990), the presently best-accepted risk value for radiation is approximately $5 \times 10^{-4}$ per rem for a risk of fatal cancer. This relationship can be rewritten to solve for the mass of DU ingested, which is associated with a lifetime fatal cancer risk of $1 \times 10^{-4}$ :

$$
1 \times 10^{-4} \text { Risk }=\left[5 \times 10^{-4} \mathrm{Risk} / \mathrm{rem}\right] \times[1 \mathrm{rem} / 15 \text { grams }] \times X,
$$

and it follows that

$$
X=3 \text { grams } .
$$

In a $70-\mathrm{kg}$ person this reduces to $0.043 \mathrm{~g} \mathrm{DU} /(\mathrm{kg}$ body weight). And to make the comparison more exact, if the intake is over a 70-year lifetime, the daily dose rate is

$$
\operatorname{Dose}_{\mathrm{DU}}=1.7 \mu \mathrm{g} /(\mathrm{kg}-\text { day }) \text {. }
$$


$\log Y=0.6011+0.7684 \bullet \log X ; R=0.77$

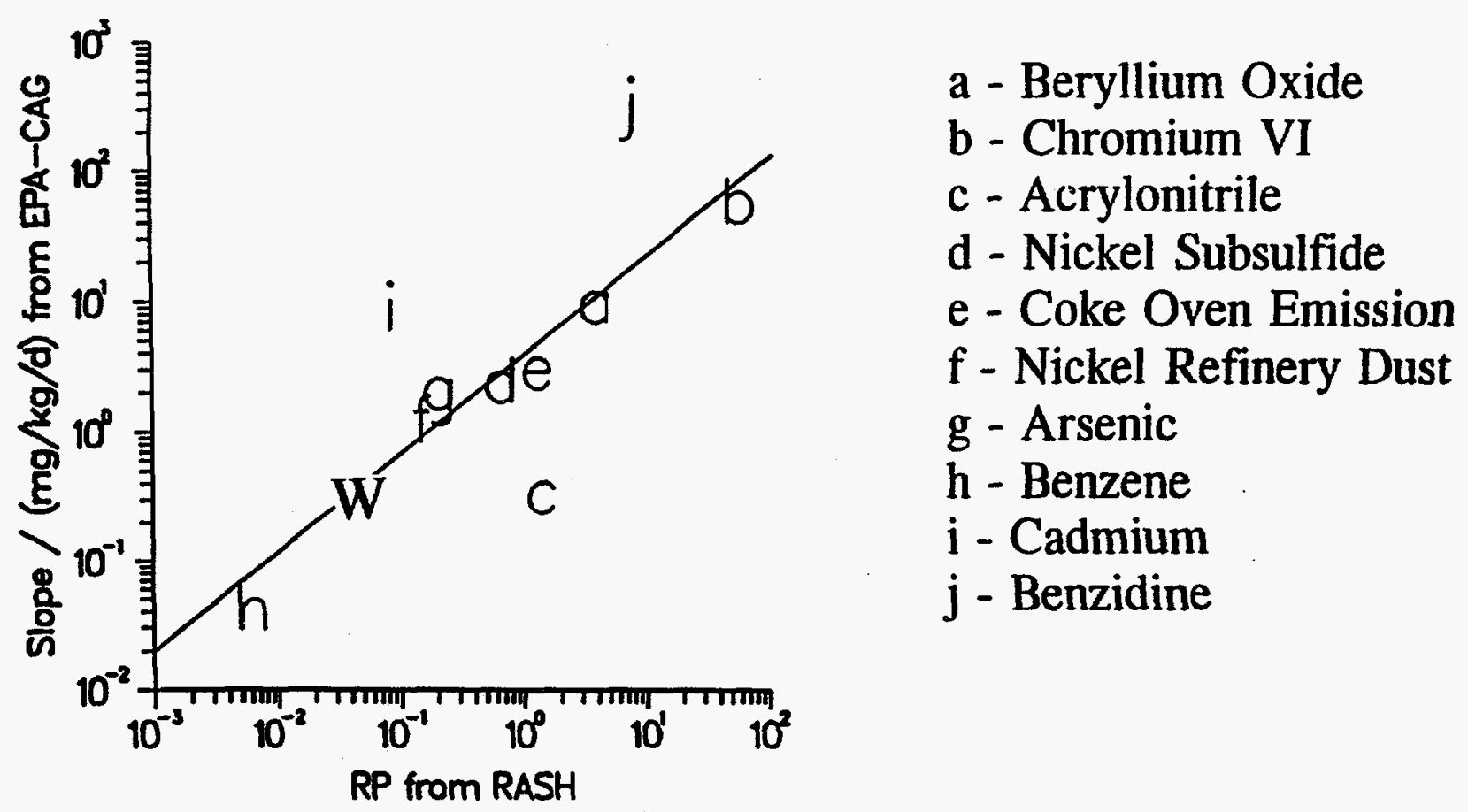

Fig. 6.2. Preliminary analysis of tungsten in the context of EPA epidemiologically derived risk factors.

This value is indistinguishable from the daily intake of tungsten, calculated above, which results in the same lifetime cancer risk, should tungsten prove to be carcinogenic at some time in the future. This analysis assumes both tungsten and DU are in environmentally mobile forms. The majority of cases of tungsten and DU entering the environment will be as penetrator rounds. These are initially metallic and relatively immobile in the environment. Mobility requires dissolution and corrosion. A preliminary synthesis of literature information suggests that for most environmental conditions tungsten will corrode at slower rates than DU. Consequently, proportionally less tungsten might be available for movement through the environment.

This relative potency approach is based on an indirect set of comparisons using two reference risk points. The first is the chemical risk based on a composite of slope factors for the epidemiologically based studies on beryllium oxide, chromium VI, acrylonitrile, nickel subsulfide, coke oven emissions, nickel refinery dust, arsenic, benzene, cadmium, and benzidine. These same materials have extensive animal and cellular data that are used to provide a link with the fundamental reference, benzo(a)pyrene. Relative potency methodology thus provides a link, by means of common bioassays, between tungsten and benzo(a)pyrene and between benzo(a)pyrene and the ten materials listed previously. The chemical side of the risk estimate then rests on the accuracy of the human risk estimate based on epidemiological studies of those ten materials and the available data for tungsten. 
The second reference used in the example of tungsten and DU is the body of information that relates cancer risk to radiation exposure. The risk measure for radiation exposure is derived from the most extensively studied agent in human history-ionizing radiation. Although there are many things yet to learn, it is most likely the relationship that will change the least during the next few decades.

\subsection{Implications of Preliminary Findings}

The full range of implications of the preceding analysis of the toxicological implications of tungsten cannot be fully assessed within the toxicological framework alone. However, with respect to health considerations, several key points can be identified.

1. It is important to understand that scientific awareness of the hazards of materials is changing. Not many years ago, the general consensus held that ostensibly chemically inert substances were nonhazardous to biological systems. But occupational exposures and the subsequent identification of occupationally induced diseases have now shown that many substances can induce a spectrum of toxicological effects including carcinogenesis. The chemically inert substances of current concern include: asbestos, calcium, coal dust, copper, copper sulfate, fiberglass, gold, graphite, iron and iron ore, platinum, quartz, sand, silica, silver, stainless steel, talc, tin, and teflon; a large percentage of these materials have produced tumors in laboratory animals in one or more studies.

2. Only a small amount of data have been accumulated for tungsten. Work within this present effort has demonstrated that disparate forms of information may be incorporated into a unified approach that builds on and integrates with the vast world knowledge on effects of toxic agents on humans. Very preliminary indications with regard to cancer are that if tungsten is found to be a carcinogen in the future, it may be roughly equivalent in hazard per unit intake to depleted uranium via the ingestion pathway. This serves to highlight the importance of understanding the corrosion behavior of tungsten and DU. Furthermore, additional data are crucial to determining whether or not this preliminary toxicity indication will stand or whether the actual nature of tungsten is very different. Two approaches might be followed: one involves the development of in vitro and in vivo data, which can be matched with reference materials as depicted in Table 6.4; a second approach involves the development of similar types of data for direct comparison of tungsten with uranium.

3. Preliminary indications suggest that noncancer effects of tungsten may be of more importance than the nephrotoxic effect of DU on renal tissue. Most of the tungsten information represents the efforts of single investigators and thus confirmation or lack of confirmation of the noncancer data is crucial.

\subsection{SPECIFIC CONSIDERATIONS FOR DU IN LIGHT OF FINDINGS}

There are numerous uncertainties or barriers associated with direct comparisons of the toxicological effects of DU with those of tungsten. For tungsten, we have used bioassay test results to compute relative potency or relative toxicity factors. Test data are very fragmentary, but the process gives a reasonable idea of the toxicological potential of tungsten relative to a wide range of chemicals ranging from compounds like ethyl alcohol and saccharine near the low end of the scale to compounds like cadmium, aflatoxin $B_{1}$, and 2,3,7,8-TCDD near the high end of the scale. This relative potency index 
also provides a means to estimate the potency of tungsten compounds to cause the same untoward effects on a dose- and potency-adjusted basis as has been observed in epidemiological studies and test animals for the reference compounds that have been studied extensively. Thus, although test data are fragmentary and there is no assurance that tungsten will in and of its own intrinsic characteristics (i.e., in the absence of mixed-field environmental and occupational exposures) actually cause effects such as cancer, we can estimate its potential to do so relative to that of better-studied compounds also tested in common bioassays.

In marked contrast, radiological compounds have historically been analyzed and modeled almost exclusively in terms of radiotoxicity. Traditionally, studies of the chemical toxicity component of radioactive compounds, even those with a long half-life, have usually been ignored. Radioactive substances are easy to study in terms of radiation dosimetry, transport, metabolism, and toxicological endpoints. In contrast, such processes are expensive and difficult to study for stable compounds unless they have radiotags. For absorption into the human body, metabolism, and distribution, most compounds are studied individually. For radiological impact on human health, most effects are modeled through the well-established concept of radiation dose. Those mathematical models are then extrapolated to low doses, long times, and large human populations to produce theoretical risk estimates.

However, at high doses in the acute exposure case, uranium has been associated with chemical renal toxicity. That class of response is generally associated with finding a "no-effect" dose threshold below which effects do not occur. For chronic toxicity associated with DU, expert committees have deduced that the most important effect is also to the kidney. Thus, uranium has and continues to be managed in terms of two theoretical considerations that address only limited aspects of its complete toxicological profile.

Uranium has a molecular weight of 238.07 , atomic number of 92 , and a $21-9-2$ outer electronic configuration. Tungsten has a molecular weight of 180.95 , atomic number of 74 , and a 32-12-2 outer electronic configuration. It seems reasonable that for consideration of relative toxicity, both substances should be tested in exactly the same way in the same biological models. Thus, the comparison of tungsten would be directly with the combined radiological and chemical aspects of DU. Currently, the two aspects, radiological and chemical, are being "weighed" on different analytical scales in spite of our attempts to improve the relative precision of hazard assessment for both substances. Future work should be directed carefully to improve the accuracy in this aspect of the life-cycle analysis. Although complex considerations of immunotoxicity, neurotoxicity, teratogenicity, and carcinogenesis are difficult to delineate in full for any substance-even benzene and especially the two substances treated here-the experimental measurements that we recommend should permit relatively precise characterization of broad toxicological foot prints for tungsten and DU. 


\subsection{REFERENCES}

Carlsen, E., et al. 1992. "Evidence for Decreasing Quality of Semen During past 50 Years," Br. Med. J. 305, 609-613.

Department of Defense: Office of the Secretary: 1987. Defense Priority Model (Environmental). Federal Register 52:44204-44206.

Department of Defense: Office of the Secretary. 1989. Defense Priority Model; Defense Environmental Restoration Program. Federal Register 54:43104-43106.

Gold, L.S., et al. 1984. "A Carcinogenic Potency Database of the Standardized Result of Animal Bioassays." Environ Health Persp. 58, 9-319.

Gold, L. S., et al. 1989. "Summary of Carcinogenic Potency and Positivity for 492 Rodent Carcinogens in the Carcinogenic Potency Database," Environ Health Persp. 79, 259-272.

Idiatullina, F. K. 1981. "Data Toward Hygienic Normalization of Tungsten in Atmospheric Air (Rus.)," Gig. Sanit., 46(9), 79-81.

International Agency for Research on Cancer. 1992. Scientific Report 116 on Mechanisms of Carcinogenesis in Risk Assessments, Lyon.

Jones, T. D. 1984. A Unifying Concept for Carcinogenic Risk Assessments: Comparison with Radiation-Induced Leukemia in Mice and Men," Health Physics. 47, 533-558.

Jones, T. D. 1991. (Invited Editorial) "On the Future of Regulating Chemicals," Environmental Auditor 2, 101-102.

Jones, T. D. 1993. "Hazardous Substances, Regulation by Government Agencies," in Handbook of Hazardous Materials, Academic Press, Inc. pp. 321-331.

Jones, T. D. 1995. "Use of Bioassays in Assessing Health Hazards from Complex Mixtures: A RASH analysis," Chemosphere 31, 2475-2484.

Jones, T. D., et al. 1983. "A Unifying Concept for Carcinogenic Risk Assessments," J. Theoret. Biol. $105,35-61$.

Jones, T. D., et al. 1979. Risk of Cancer Based on Promotion from Cytotoxicity. National Bureau of Standards Special Publication 554, p. 41, Washington D.C. U.S. Department of Commerce.

Jones, T. D., et al. 1986. Animal Mortality Resulting from Uniform Exposures to Photon Radiations: Calculated $L D_{50}$ s and a Compilation of Experimental Data, ORNL/TM 6338, Oak Ridge National Laboratory.

Jones, T. D., et al. 1991. "Protection of Human Health from Mixtures of Radionuclides and Chemicals in Drinking Water," Arch of Environ Contam and Toxicol 20, 143-150. 
Jones, T. D., et al. 1991. "Chemical Pollutants: A Caricaturized Logos for Future Planning," Environmental Auditor 2, 71-88.

Jones, T. D., et al. 1988. "Chemical Scoring by a Rapid Screening of Hazard (RASH) Method," Risk Analysis 8, 99-118.

Jones, T. D., et al. 1985. "Permissible Concentrations of Chemicals in Air and Water Derived from RTECS Entries: A "RASH” Chemical Scoring System," Toxicol. Indust. Health 1, 213-234.

Jordan, C., et al. 1990. "Memory Deficits in Workers Suffering from Hard Metal Disease," Toxicol. Lett. 54, 241-243.

Kaplun, Z. S., and Mezentseva, N. V. 1959. "Hygienic Evaluation of Aerosols Formed in the Manufacture of Hard Alloy (Rus)," Gig. Sanit. 24, 16-22.

Levin, H. S., et al. 1976. "Anxiety Associated With Exposure to Organophosphate Compounds," Arch. Gen. Psychiatry 33, 225-228.

Metcalf, D. R., and Holmes, J. H. 1969. "EEG, Psychological, and Neurological Alterations in Humans with Organophosphorus Exposure," Ann. N.Y. Acad. Sci. 160, 357-365.

Munro, N. B., et al. 1994. "Toxicity of the Organophosphate Chemical Warfare Agents GA, GB, and VX: Implications for Public Protection," Environ. Health Perspect. 102, 18-38.

Nadeenko, V. G., et al. 1977. "New Data for Standardization of Tungsten and Molybdenum in their Separate and Simultaneous Presence in Water Bodies (Rus.)," Gig. Sanit. 3, 7-11.

Nadeenko, V. G. 1966. "Maximum Permissible Concentrations of Tungsten in Water Basins (Eksperimental'nye dannye $\mathrm{k}$ obosnovaniyu predel'no dopustimoi kontsentratsii vol'frama v vode vodoemov)," Hyg. Sanitat. 31, 197-204.

Nadeenko, V. G. and Lenchenko, V. G. 1977. "The Nature of the Combined Action of Small Doses of Certain Elements-Antagonists (Rus.)," Gig. Sanit. 8, 30-34.

Nadeenko, V. G., et al. 1978. "The Influence of Tungsten, Molybdenum, Copper, and Arsenic on the Intrauterine Development of the Fetus (Rus.)," Farmakol. Toksikol. 41, 620-623.

Savage, E.P., et al. 1988. "Chronic Neurological Sequelae of Acute Organophosphate Pesticide Poisoning," Arch. Environ. Health 43, 38-45.

Sharpe, R. M. and Skakkebaek, N. E. 1993. "Are Oesterogens Involved in Falling Sperm Counts and Disorders of The Male Reproductive Tract," Lancet 341, 1392-1395.

Sidell, F. R. 1992. "Clinical Considerations In Nerve Agent Intoxication," Chemical Warfare Agents, ed. S. Somani, Academic Press, N.Y., 155-194. 
Sidell, F. R. and Groff, W. A. 1974. "The Reactivatibility of Cholinesterase Inhibited by VX and Sarin in Man," Toxicol. Appl. Pharmacol. 27, 241-252.

Vengerskaya, K. Y. and Salikhodzhaev, S. S. 1962. "Some Problems Relating to the Effects of Tungsten Powder on Humans," Gig. Tr. Prof. Zabol. 6, 27-29.

Wide, M., et al. 1986. "Distribution of Tungstate in Pregnant Mice and Effects on Embryonic Cells In Vitro," Environ. Res. 40, 487-498. 



\section{SIMULATION OF ENVIRONMENTAL EXPOSURE SCENARIO}

The issue addressed in this report is the question "Is there any difference in environmental impacts between tungsten and depleted uranium as used for armor penetrators?" Our approach in the previous chapters has been to carry out, within the structural framework of mathematical modeling, a synthesis of the information regarding the behavior of tungsten in the environment and in the body and to couple that modeling with modeling of tungsten's toxicological characteristics. We have not carried out an evaluation of the relative release of these two contaminants, and thus the discussion herein is not in absolute units.

Chapters 4 through 6 summarize the information regarding the fate of tungsten in the environment, in man, and its potential toxicity. In this chapter, a preliminary exposure scenario is constructed to estimate the potential effects on humans. To avoid the complexities of various release scenarios, an equal mass of the two principal KEP materials is assumed to be deposited in the soil, and soluble forms are generated at unequal rates for tungsten and DU. This rationale for the initial point of the scenario comes from an interest in evaluating environmental impacts of the two alternatives on a common test range and roughly approximating the unequal rates of formation of mobile forms that might be expected to occur in the environment.

\subsection{CHRONIC EXPOSURE SCENARIO}

As metal, there will be essentially no uptake into plants and into the food chain. Movement through the food chain takes place only after the metal is converted to a soluble chemical form and transported in the soil water. With an equal mass of tungsten and DU deposited in the soil, the rate at which solubilization of the materials occurs in the soil becomes important. These rates will determine the relative concentrations of the materials that are available for plant uptake and movement within the food chain, as discussed in Chapter 4. The ultimate exposure levels to people and potential health impacts are directly proportional to the solubilization rates. The literature on relative dissolution in weak acids found in soils is very sparse. However, some tungsten compounds are known to be very corrosion resistant, while some uranium compounds are quite mobile in the environment. For illustrative purposes, we will assume that DU is 100 times more soluble in the soil than tungsten. Thus, if the quantity of uranium available for plant uptake is 100 times greater than tungsten, the relative effects will be different than if uranium and tungsten dissolve/oxidize/transport at equal rates. Because of the importance of the process in establishing the relative concentrations available for transport in the environment, further information on the solubility of both materials in the environment is needed. However, synthesis of some measurements documented in the literature suggest that this relative rate between DU and tungsten may be as high as 1:100. This scenario is carried out with this assumption and the inhalation and ingestion calculations as shown subsequently.

Inhalation-Airborne dust levels in a rural area are on the order of $10^{-7} \mathrm{~kg} \mathrm{~m}^{-3}\left(10^{-3} \mathrm{~g} \mathrm{~m}^{-3}\right)$. The concentration of tungsten and DU in the soil at $10 \mathrm{ppm}$ results in intake rates of $2.2 \times 10^{-8} \mathrm{~g} \mathrm{~d}^{-1}$, respectively, assuming that man takes air at a rate of $22 \mathrm{~m}^{3} / \mathrm{d}$.

Ingestion-The uptake of tungsten and DU from the soil into vegetation may occur through root uptake as discussed in Chapter 4 . Concentration factors are used to relate the concentration of the material in the edible portion of the plant to the concentration in soil. In Chapter 4 , concentration 
factors of 0.3 and 0.009 were stated for tungsten and DU (see Table 4.4). We assume that the solubilization of tungsten is one hundredth that of DU, and hence the soil concentration of $10 \mathrm{ppm}$ results in soluble concentrations of 0.1 and $10 \mathrm{ppm}$, respectively. Table 4.1 indicates a consumption rate for vegetation of $190 \mathrm{~kg} \mathrm{y}^{-1}$ or about $0.52 \mathrm{~kg} \mathrm{~d}^{-1}$. Following Equation (4.1), the intake rate of tungsten is given as

$$
\dot{I}=C_{\text {soil }} C F_{\text {plant }}^{\cdot} I_{\text {plant }}
$$

where $\dot{I}$ is the rate of intake of tungsten in vegetation, $C_{\text {soil }}$ is the concentration of soluble tungsten in soil (0.1 ppm), $C F_{\text {plant }}$ is the concentration factor for tungsten, and $I_{\text {plant }}$ is the rate of consumption of vegetation. The $C F_{\text {plant }}$ is defined in terms of the dry weight of the vegetation while the consumption rate $I_{\text {plent }}$ is stated in terms of wet weight. We assume a wet to dry weight ratio of 4 to 1 . The rate of ingestion of tungsten in vegetation is thus

$$
\begin{aligned}
\dot{I} & =C_{\text {soil }} C F_{\text {plant }} I_{\text {plant }} \\
& =10^{-7} \times 0.3 \times 520 / 4 ; \\
& =3.9 \times 10^{-6} \mathrm{gd}^{-1}
\end{aligned}
$$

and for DU

$$
\begin{aligned}
I & =C_{\text {soil }} C F_{\text {plant }} I_{\text {plant }} \\
& =10^{-5} \times 0.009 \times 520 / 4 \\
& =1.2 \times 10^{-5} \mathrm{gd}^{-1}
\end{aligned}
$$

The intake of tungsten and DU in vegetation by livestock results in its accumulation in milk and meat products. The equations used to evaluate this transfer are given in Chapter 4 . The equation can be written as

$$
\begin{aligned}
C_{\text {meat,milk }} & =C_{\text {plant }} Q_{\text {jeed }} B T F_{\text {meat,milk }} \\
& =C_{\text {soil }} C F_{\text {plant }} Q_{\text {feed }} B T F_{\text {meat,milk }}
\end{aligned},
$$

where $C_{\text {meat,milk }}$ is the concentration of the material in meat or milk, $C_{p l a n t}$ is the concentration in the vegetation consumed by the animal, $Q_{\text {feed }}$ is the rate the animal is fed vegetation, and $B T F_{\text {meat,milk }}$ is the biotransfer factor for the animal product. All other quantities are as defined previously. See Table 4.4 for the values of these parameters for tungsten and DU. Assuming the animals are fed vegetation at a rate of $10 \mathrm{~kg} \mathrm{~d}^{-1}$ and that the human intake of milk and meat are as stated in Table 4.1, then the following ingestion rates of tungsten and DU are indicated:

tungsten: $\quad 1.8 \times 10^{-8} \mathrm{~g} \mathrm{~d}^{-1}$ in milk and $2.1 \times 10^{-6} \mathrm{~g} \mathrm{~d}^{-1}$ in meat DU: $\quad 5.4 \times 10^{-8} \mathrm{~g} \mathrm{~d}^{-1}$ in milk and $1.2 \times 10^{-5} \mathrm{~g} \mathrm{~d}^{-1}$ in meat 
The following total intake rates are estimated for this scenario:

$\begin{array}{ll}\text { tungsten: } & \text { Inhalation: } 2.2 \times 10^{-10} \mathrm{~g} \mathrm{~d}^{-1} \\ \text { Ingestion: } & 6.0 \times 10^{-6} \mathrm{~g} \mathrm{~d}^{-1}, \\ \text { DU: } & \text { Inhalation: } 2.2 \times 10^{-8} \mathrm{~g} \mathrm{~d}^{-1} \\ \text { Ingestion: } & 1.2 \times 10^{-5} \mathrm{~g} \mathrm{~d}^{-1}\end{array}$

Using the data of Table 5.4, the above intake rates would result in the following tissue levels of tungsten and DU:

Table 7.1. Tungsten and depleted uranium levels

in the body resulting from chronic exposure

to $10.0 \mathrm{ppm}$ of tungsten and $\mathrm{DU}$ in soil

\begin{tabular}{lcc}
\hline Organ & Tungsten $(\mathrm{g})$ & Uranium $(\mathrm{g})$ \\
\hline Skeleton & $1.8 \times 10^{-4}$ & $6.0 \times 10^{-5}$ \\
Liver & $7.9 \times 10^{-6}$ & $1.8 \times 10^{-6}$ \\
Kidneys & $9.4 \times 10^{-4}$ & $8.0 \times 10^{-7}$ \\
Spleen & $9.3 \times 10^{-7}$ & \\
Other Tissues & $6.7 \times 10^{-6}$ & $2.2 \times 10^{-5}$ \\
\hline
\end{tabular}

The tungsten levels in systemic tissues tend to be greater than the corresponding uranium values. This is a result of the higher environmental uptake of mobile tungsten into plants and food chain animals and its greater fractional absorption from the GI tract.

\subsection{HUMAN HEALTH RESPONSES TO EXPOSURE SCENARIO}

The scenario analysis has been based on equal amounts of DU and tungsten in the soil of a practice range and a factor of 100 greater solubilization of DU in the soil. A single combined inhalation and ingestion pathway was developed. Once in the mobile form, the primary factors in this scenario that influence the movement of tungsten and DU within the food chain are the concentration factors going from soil to plant, as discussed in Chapter 4, and then the GI tract absorption factor for human ingestion, known as the $f_{1}$ factor, described in Chapter 5 .

For both of these factors, tungsten is significantly more mobile than DU. That is, the plants and the GI tract seem to be able to exclude uranium with greater efficiency than tungsten. But from preliminary information collected from the literature, uranium becomes available for environmental movement at a greater rate than tungsten. For this scenario, a difference of 100 times is assumed; environmental mobilization data on anticipated alloys will be necessary to refine this and any future scenario. This simple pathway analysis results in daily intake rates of $6.0 \times 10^{-6} \mathrm{~g} / \mathrm{d}$ for tungsten and $1.2 \times 10^{-5}$ for DU. For a $70-\mathrm{kg}$ adult human, these figures are then converted to dose rates of $0.000085 \mathrm{mg} /\{\mathrm{kg}$-day $\}$ for tungsten and $0.00017 \mathrm{mg} /\{\mathrm{kg}$-day $\}$ for DU. These daily doses are used in the following to assess the impact of potential toxicities of tungsten and DU. 


\subsubsection{Noncancer Toxicities}

The traditional noncancer health impact of uranium is renal (kidney) toxicity as described in Appendix D. Toxic effects on the kidneys are assumed to occur only when the uranium concentration in the kidney tissue exceeds some threshold level. Since the early $1950 \mathrm{~s}$ a concentration of $3 \mu \mathrm{g}$ of uranium per gram of kidney tissue has served as a primary guidance level for avoidance of chemical toxicity. Recently, several reviewers and analysts have proposed that the longstanding chemical guidance level for uranium be reduced, particularly for consideration of chronic exposure, as in the case of the scenario under consideration. For long-term environmental exposures, the value used is $0.3 \mu \mathrm{g}$ of uranium per gram of kidney tissue (as in Appendix D).

From Figure 4 of Appendix D, an annual intake of $5 \mathrm{~g}$ of DU results in a kidney concentration of $0.3 \mu \mathrm{g} \mathrm{DU} / \mathrm{g}$ kidney tissue. An annual intake of $5 \mathrm{~g}$ reduces to a daily dose of $0.00019 \mathrm{mg} /\{\mathrm{kg}$-day $\}$. This is essentially the same value as the daily dose of DU that results from the scenario beginning with $10 \mathrm{ppm} \mathrm{DU}$ in the soil, that is, $0.00017 \mathrm{mg} /\{\mathrm{kg}$-day $\}$. Thus, this intake corresponds to a conservative no-observed-adverse-effects-level (NOAEL) for renal toxicity.

Beginning with $10 \mathrm{ppm}$ tungsten in the soil, the scenario results in a daily dose of $0.000085 \mathrm{mg} /\{\mathrm{kg}$ day\}. This is below the most sensitive health endpoints for laboratory animals identified in Chapter 6, which are teratogenic and reproductive effects and possibly neurotoxicity. According to discussions in Chapter 6, teratogenic, reproductive, and suggestions of neurotoxic effects can be induced by a chronic tungsten administration of $0.005 \mathrm{mg} /\{\mathrm{kg}$-day $\}$ to laboratory animals. This dose rate is the lowest dose rate tested in the laboratory studies for these endpoints. Thus, based on a preliminary analysis, and using data from the literature, the scenario chosen will result in a dose of tungsten that is less than that which induced the most sensitive noncancer health endpoints that are identified in the literature. The present scenario would predict neither renal toxicity from the projected DU ingestion level nor noncancer effects from ingestion of tungsten.

\subsubsection{Potential for Cancer}

As described in Chapter 6, the literature does not contain reports describing tungsten as either a carcinogen or a noncarcinogen. A significant effort was expended within Phase I, to utilize the diverse types of laboratory animal information, to estimate a potential cancer potency for tungsten. This potential cancer potency could then be compared with the cancer potency of DU, which is based entirely on calculated cancer risk from radiation dose. The work summarized in Chapter 6 defines the approximate potency of tungsten if it were at some time in the future found to be a carcinogen. It is with that potency projection that the potential cancer risk of tungsten is compared here with that of uranium.

From discussions in Sect. 6.5, a daily tungsten dose of $0.000086 \mathrm{mg} /\{\mathrm{kg}$-day $\}$ could, if tungsten is found to be a carcinogen, result in a lifetime cancer risk of $4.5 \times 10^{-6}$. The daily DU dose of $0.00017 \mathrm{mg} /\{\mathrm{kg}$-day $\}$ results in a lifetime cancer risk of $1.0 \times 10^{-5}$. This is about two times greater than the risk estimated for tungsten. Thus, in this scenario beginning with $10 \mathrm{ppm}$ of DU and tungsten in the soil, we see that DU may result in a potential cancer risk about twice that of tungsten, should it be found to be a carcinogen. 


\subsection{SUMMARY}

This analysis is based on equal amounts of tungsten and DU deposited in the soil of a test range. The relative rate by which the two primary materials can transform from metal to an oxide or other environmentally mobile form and move within the soil water is not known with certainty at this time. Based on preliminary information collected from the literature, we assumed that DU converts to a mobile state 100 times faster than tungsten. Analysis of this scenario indicates that both from the standpoint of health effect endpoints, tungsten may pose a relatively lesser human hazard than DU. However, that conclusion or one developed from alternative quantity/solubility assumptions cannot be stated with certainty because the calculations are not based on experimental data for KEP materials. In this analysis, the toxic effects of uranium were limited to the effects addressed in current federal guidance for exposure to uranium, that is, the potential chemical toxicity of uranium to kidneys and the potential carcinogenic effects of uranium from radioactivity. Although there is little information on other, more subtle effects of uranium, the extant health effects data base for tungsten is much smaller than that for DU.

Firm conclusions for any scenario cannot and should not be drawn with the present data. This scenario has used all types of information gathered during the course of Phase $\mathrm{I}$, but no element of this data base has been sufficiently verified to support important decisions. Uncertainties exist on the relative environmental mobility rates for tungsten and DU, on the relative uptake factors into the food chain, the relative uptake factors once in the human body, and the relative toxic effects of noncancer and cancer health effects. The work, however, demonstrates that a sufficiently detailed accounting of the potential health impacts of tungsten with respect to DU have been constructed to support scenario evaluations with better data. Specific areas that require augmented data have been identified and are discussed in Chapter 8. 


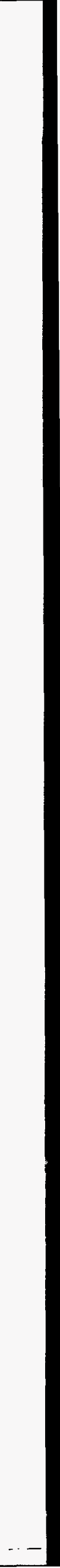




\section{CONCLUSIONS, RECOMMENDATIONS, AND SUMMARY}

Conclusions presented in this chapter reflect a phased approach to the program. This phased approach is based on the premise that an analysis of a complex problem such as the life-cycle evaluation of alternative materials for heavy armor KEPs must proceed along a critical path. We determined early on that the critical path included basic knowledge about tungsten behavior in the environment. Given that the program goals included the evaluation of environmental and health risks from alternative KEP materials, we had to be in a position to evaluate those risks. If the data base for evaluation of those risks were inadequate, this would have to be identified early in the program so that development of the data base could proceed in a timely fashion for adequate decision making in the life-cycle analysis. Thus, this first year of the program focused much of the available resources on a critical analysis of the extant relevant environmental and biological data using a simulation framework set up to analyze relevant experimental data as they become available. This critical analysis has resulted in the assembly of current knowledge about the movement and distribution of tungsten in the environment, the human body, and its potential effects on human health. Concurrently, we identified a number of important areas where additional information is needed. The major conclusions of this first year's work and directions anticipated for future work follow.

\subsection{CONCLUSIONS FROM ENVIRONMENTAL SIMULATION MODELING RESULTS}

These conclusions focus on the major elements of the work in the preceding seven chapters. In developing an analysis of the available data, all work was done from the perspective of how we would be able to use our results in an environmental analysis. The elements of an environmental analysis are essentially three:

1. How does a material behave in the environment as it moves from a point of introduction into the environment toward an ultimate intake by humans?

2. How is the material retained and distributed within the human body once taken in?

3. What happens to the human who takes in the material under study?

The conclusions that follow that pattern are discussed as follows.

\subsubsection{Environmental Pathways}

The entire data base supporting the bioaccumulation and environmental transfer factors for tungsten and uranium is quite small. From the information available, tungsten is shown to be relatively more mobile in the soil than uranium. Tungsten is also more easily taken into plants than uranium. Soilwater partition coefficients for tungsten and uranium suggest that uranium's mobility to go from soil to water is only about $7 \%$ of the mobility of tungsten. Moreover, a similar difference is indicated in the movement of uranium and tungsten from soil to the edible parts of plants growing in soil contaminated with these two elements. Assuming equal concentrations in the soil, tungsten uptake into plants may be 33 times greater than that for uranium. Other differences in the transfer of these two elements within the food chain are not so pronounced. Taken together, these findings suggest that gram for gram, tungsten will move more extensively in the soil than uranium and that it will be found to be more highly concentrated in plants than uranium, but differences in corrosion rates of the two metals may influence the quantity of each that is available in the soil to be transported in the environment. 


\subsubsection{Absorption, Retention, and Distribution Within the Human Body}

Based on the modeling of the biokinetics of tungsten (Appendix C) and knowledge of uranium, a better understanding of the differences in movement between tungsten and uranium has been developed. At present, the most important information is that given an equal gram intake by ingestion, Type $M$ tungsten is absorbed by the GI tract about 25 times more efficiently than uranium. Very little difference in retention is seen for the inhalation pathway, but inhaled tungsten is distributed differently within the body than uranium. These results are preliminary and are based on the scattered data from large animals and on data from rats when no other data were available. However, the rat may not be a good model for humans.

\subsubsection{Health Impacts}

A variety of noncancer health impacts were identified from literature reports on animal and cellular studies. Important impacts may involve neurotoxicity, reproductive toxicity and developmental toxicity, or teratogenesis endpoints. Effects on some of these endpoints were produced using exposure levels that may approximate environmental exposures.

With respect to possible cancer effects, no cancer studies were identified that designated tungsten as a noncarcinogen or a carcinogen at a $95 \%$ statistical confidence level. On the basis of very sparse data, if tungsten is found to be a carcinogen, its potency would be around 0.04 relative to benzo(a)pyrene. In practical applications of comparing tungsten to DU, if an objective were to maintain a lifetime risk from continuous exposure at or below $1 \times 10^{-4}$ level, the associated dose rates for tungsten and DU must be: Dose $_{\mathrm{w}}=1.9 \mu \mathrm{g} /\{\mathrm{kg}$-day $\}$ and Dose $_{\mathrm{Du}}=1.7 \mu \mathrm{g} /(\mathrm{kg}$-day $)$ or lower, respectively. The relative potency projections suggest that gram for gram, tungsten and DU may be about equivalent in toxicological potency when exposure is via the ingestion route. This analysis does not demonstrate that tungsten is a carcinogen. The calculations are hypothetical and direct us to specific tests that are needed to verify or dispel what may be fragile estimates of statistical parameters associated with solubility, uptake, and risk from tungsten KEP fragments. These experiments and tests are outlined in Section 8.3 following a brief summary of progress and accomplishments in this Phase I study.

\subsubsection{Summary of Phase I Accomplishments}

The conclusions and recommendations from this study must be understood in the context of current military and industrial operations. The initial toxicological assessment of tungsten was performed with readily available data from the literature, instead of data developed for our analysis. Out of this Phase I study, a significant number of new analyses were completed, new models were developed, and new perspectives were achieved. They include:

- reevaluation and development of new, more reliable values for soil concentration and biotransfer factors for tungsten;

- assembly of values for environmentally significant factors necessary for quantitative analysis of tungsten movement in soil and through the food chain;

- development of a physiologically based biokinetic model of tungsten absorption, distribution, and elimination in humans;

- selection of an improved value of the fractional GI absorption for tungsten;

- comparison of biokinetics of tungsten and DU in humans based on acute exposure; 
- quantitative prediction of tissue content of tungsten and uranium given lifetime exposure by inhalation and ingestion of daily unit intakes;

- identification of new, no-observed-adverse-effects tissue level for chronic renal toxicity of uranium;

- identification of tungsten toxicity data for noncancer endpoints that warrant further experimental evaluation as they may represent effects of more concern than DUs potential for renal toxicity; and

- development of scenario methodology using quantitative environmental transfer factors, biokinetic models of tungsten and DU, relative potency analysis results, and revised NOAEL tissue DU level for renal toxicity to quantitatively compare movement of DU and tungsten from soil contamination to human tissues and evaluate the comparative health impacts.

\subsection{RECOMMENDATIONS FOR FOLLOW-UP EXPERIMENTS AND TESTS}

Based on quantitative analyses of the available data, specific additional data are needed to reduce the uncertainties in a comparative assessment of the environmental effects of tungsten and uranium.

\subsubsection{Environmental Pathways}

The most important environmental factors for tungsten relative to uranium are the relative corrosion rates, the soil-to-water partition coefficients, plant uptake factors, and plant-to-animal transfer factors. The present data base, however small, supports factors that consistently suggest that tungsten is significantly more mobile and accessible to organisms than uranium. Because of the importance of these factors in the calculations of contaminant transport to humans, targeted studies are recommended to better document soil-to-water partition coefficients, plant uptake factors, and plant-to-animal transfer factors.

\subsubsection{Absorption, Retention, and Distribution Within the Human Body}

Knowledge about absorption, retention, and distribution within the body are key factors in determining the degree and type of toxicity of a toxic agent. With regard to inhalation, there has been only one reasonably detailed study of the biokinetics of inhaled tungsten, and it involved only one physiochemical form of tungsten. Further investigation leading to the identification of the representative forms of airborne tungsten that may be encountered in environmental, occupational, or military settings as needed. Additional information on the level of absorption of inhaled tungsten as needed. Such information might be gained from easily accessible measurements such as urinary excretion of tungsten in workers acutely exposed to tungsten in air.

Data on GI uptake of tungsten in different types of animals suggest that the fractional absorption can vary substantially from one situation to another depending on a variety of factors including age. Our data on GI absorption come from several animal species, but we have no human data. Because soft tissues are indicated as potential sites for toxic action by tungsten, some effort is needed to resolve the larger uncertainties in predictions of the systemic model that are associated with soft tissues. 
Given the need to improve understanding of the biokinetics of tungsten in humans, it is recommended that a two-pronged approach be used. First, build on the available data base for rats with future experiments on rats and second, perform more limited parallel experiments on larger animals. If the comparative data suggest that the rat is an inappropriate model for larger animals, then a better model should be found.

\subsubsection{Health Impacts}

Because the commercial use of tungsten has been limited in the past, with most human exposures to forms combined with cobalt, harmful effects that have been observed have been mostly attributed to cobalt. Moreover, a few laboratory studies have been conducted, but those few have not yielded alarming data for tungsten. Finally, many studies have been one of a kind. Consequently, we have substantial uncertainty in the information available for evaluation of potential health impacts. From an overall perspective, it is far more accurate to note that tungsten has been inadequately tested than to conclude that tungsten is of low toxicity and a noncarcinogen.

\subsubsection{Noncancer Endpoints}

With noncancer toxicity, we have identified three classes of effects that are important for further evaluation: neurotoxicity, reproductive toxicity, and teratogenicity. It is of high importance to determine whether tungsten exposure has the potential to disrupt reproductive function in either the female or male. Indicators of developmental toxicity, like other studies, consist mainly of unreplicated works, although several experiments in the same laboratory gave consistent results on skeletal teratogenicity. As with neurotoxicity and reproductive toxicity, the evidence for tungsten developmental toxicity, if confirmed, would be of much greater significance than the kidney toxicity associated with low-dose uranium exposure. Furthermore, combinations of tungsten and other materials for potential alloys should be tested for increased toxicity in view of the enhanced toxicity seen with tungsten-cobalt combinations in hard-metal alloys.

\subsubsection{Cancer}

Obtaining a better understanding of the potential for inducing cancer is an important follow-up to this study. Analysis of its potential cancer potency put tungsten at about the same level as DU, gram for gram, for ingestion. Relative to chemicals of commerce, tungsten seems to have the approximate potency of the human carcinogens benzene, coke oven emissions, and nickel refinery dust. This conclusion is derived from the results of a relative potency analysis that made use of various types of data on measures of tungsten toxicity other than carcinogenicity. The potency derived is only a measure of toxicological potency that would approximately define the potency of tungsten, if it were determined to be a carcinogen in the future.

The data upon which the cancer potency evaluations are based are very limited. In the next phase of this program, strengthening the basis for relative potency analysis and developing the data necessary to to make direct comparisons between DU and tungsten are recommended. Performing biological experiments with tungsten in a variety of biological tests can be done rather quickly and cost effectively. 


\subsection{SPECIFIC CONSIDERATIONS FOR DU IN LIGHT OF FINDINGS}

There are numerous uncertainties or barriers associated with direct comparisons of toxicological effects from DU with those from tungsten. Exposure to tungsten is associated with a complex continuum of response endpoints ranging from production of subcellular lesions, through enzymatic changes, pathological harm to cells or tissues, to possible cancer and death from both acute and chronic toxicity. Although radiotoxicity and chemical toxicity can both be analyzed on that same continuum, the two disciplines have evolved independently, and measures of toxic injury cannot be treated realistically and directly from the test data that are currently available. Currently, the two aspects, radiological and chemical, are being "weighed" on different analytical scales. Future work should be directed carefully to improve the accuracy and reduce uncertainties in comparing the chemical potency of tungsten and DU for use in material life-cycle analysis.

\subsection{PRIORITIES FOR FILLING DATA GAPS}

Finally, we conclude that, although these analyses indicate that tungsten's environmental behavior and health effects may not be as benign as previously assumed, DU may also be less well studied than is generally appreciated. Thus, although tungsten could be adopted as a base KEP material with appropriate management procedures for some parts of the life cycle, the data on both tungsten and DU are insufficient for purposes of choosing between them on an environmental basis. Gathering additional environmental and toxicological data on both substances is essential to support informed decision making. The most important data gaps in their order of importance for basic data collection are as follows.

- The relative rates of corrosion and formation of environmentally mobile compounds by tungsten and DU. Is the rate of conversion into soluble form for tungsten KEP fragments 1/100th that of $D U$ ?

- Differences in tungsten and DU with respect to soil-to-water partition coefficients, plant uptake factors, and plant-to-animal transfer factors. Is the plant concentration factor for mobile tungsten emanating from KEP fragments 35 times larger than for $D U$ ?

- Identification of representative forms of airborne tungsten in environmental, occupational, and military settings. What is the measured median aerodynamic diameter for tungsten and $D U$ aerosols from KEPs, and do these fall in the range of 0.1 to $100 \mu \mathrm{m}$ ?

- Chemical potency of DU compared to projections for tungsten developed in Phase I and direct comparisons of the two materials in specific biological experiments. Are tungsten and $D U$ about equal in chemical potency, and is a dose of $1.9 \mu \mathrm{g} /(\mathrm{kg}$-day/ the appropriate level for maintaining no-observed-adverse-effects from a lifetime of continuous exposure to tungsten? What is the corresponding nonradiologically based dose for no observed effects from $D U$ ?

- The toxicological effects of alloying materials in combination with tungsten and DU. Will the presence of alloying materials in KEP rounds result in conditions favorable to possible synergistic effects as observed with tungsten-cobalt combinations? 
- Refined estimates of absorption of tungsten through inhaled and ingested pathways based on predominant forms of $\mathrm{DU}$ and tungsten identified as mobile products of corrosion. Is the predominant form of tungsten and DU associated with spent KEP rounds of type $M$ solubility or are the two materials different in solubility characteristics? Is absorption of tungsten originating from KEP fragments $50 \%$ greater than DU, or is a different value in the 5 to $75 \%$ range more appropriate?

\subsection{CONCLUSIONS FOR POLLUTION PREVENTION INITIATIVES}

A significant fraction of the available data are suggestive of tungsten being active toxicologically at environmentally relevant levels although perhaps less so than DU. This early observation must be understood with respect to the wide range of hazards encountered in military and industrial applications. Decisions about future use thus represent a balance between materials properties and lifecycle costs. This work has begun the ground work to support an environmental material life-cycle analysis of tungsten and has determined that if tungsten is chosen as a KEP material, it may require management as a potential hazard particularly if alloys are toxicologically less benign. Exactly how the treatment should be changed awaits the development of better data in light of other materials that might serve its functions. Since the major "other" material is DU, it should be investigated in parallel with data collection efforts for evaluating the material management life cycle of tungsten. Basic research experiments and field tests are the next steps in addressing data gaps, improving parameter estimates, and verifying potency projections that are presented in the preceding chapters of this report. Better experimental data in tandem with the environmental modeling framework will provide the KEP-user community with the information it needs to better incorporate environmental strategy into mission performance. 
APPENDIXES 



\section{INTRODUCTION TO THE APPENDIXES}

Assessing the environmental acceptability of high-performance alternatives to depleted uranium penetrators involves many interrelated issues. The basic issues include (1) properties of the materials for mission performance, (2) environmental mobility of materials introduced into air, land, and water, (3) effects on plants and animals, and (4) life-cycle costs of managing the materials from cradle to grave.

Within Phase I of this multiyear program, we concentrated on the most important unknowns in the critical path for life-cycle assessment. They are: (1) environmental transport factors for tungsten, (2) distribution of tungsten within the human body after an initial exposure, and (3) the toxic effects of tungsten. The appendixes in this volume begin with a presentation of bibliographical references from which selected data were collected for use in the simulation modeling and scenario analysis presented in Volume I. The simulation modeling framework and related topics are presented in Appendixes $\mathrm{C}$ through $\mathrm{G}$. In Appendix $\mathrm{C}$, a biokinetics model for tungsten is defined by developing three submodel components. These submodels include: (1) respiratory tract, (2) gastrointestinal tract, and (3) systemic tungsten. Limits on intake of uranium based on radiological and chemical guidance are presented in Appendix D. In Appendix E, the authors use the information from Appendixes C and D to compare the biokinetics of tungsten and uranium in adult humans. Potential health effects and methods for filling data gaps are discussed in Appendix F ("Noncancer Health Effects") and Appendix G ("Methods for Estimating Cancer Potency of Tungsten in Humans"). The next phase of this study will involve basic research experiments and field tests to address the data gaps that are highlighted in these appendixes. 


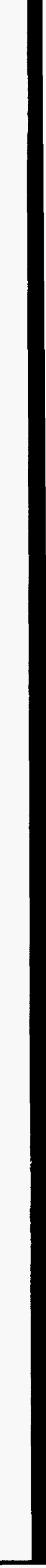




\section{APPENDIX A}

BIBLIOGRAPHY ON TUNGSTEN WITH EMPHASIS ON ENVIRONMENTAL INFORMATION 


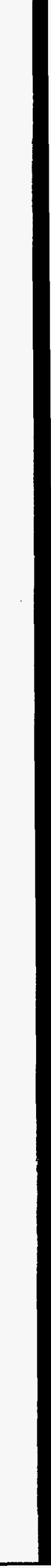




\section{APPENDIX A. BIBLIOGRAPHY ON TUNGSTEN WITH EMPHASIS ON ENVIRONMENTAL INFORMATION}

Aamodt, R. L. 1973 "Retention and Excretion of Injected ${ }^{181}$ W Labeled Sodium Tungstate by Beagles," Health Physics 24, 519-524.

Aamodt, R. L. 1975. "Inhalation of ${ }^{181}$ W Labeled Tungstic Oxide by Six Beagle Dogs," Health Physics $28,733-742$.

American Conference of Governmental Industrial Hygienists (ACGIH) 1994. 1994-1995 Threshold Limit Values for Chemical Substances and Physical Agents and Biological Exposure Indicies, American Conference of Governmental Industrial Hygienists, Cincinnati.

American Conference of Governmental Industrial Hygienists (ACGIH) 1986. Documentation of the Threshold Limit Values and Biological Exposure Indices, 5th ed., American Conference of Governmental Industrial Hygienists, Cincinnati.

American Conference of Government Industrial Hygienists. Threshold Limit Values and Biological Exposure Indices for 1989-1990. 6500 Glenway Ave. Bldg. D-7. Cincinnati, OH 45211-4438.

Aguas, A. P., N. R. Grande, and E. Carvalho 1991. "Inflammatory Macrophages in the Dog Contain High Amounts of Intravesicular Ferritin and Are Associated with Pouches of Connective Tissue Fibers," Am. J. Anat. 190, 89-96.

Ando, A. et al., 1989. "Relation between the Location of Elements in the Periodic Table and Various Organ-Uptake Rates," Nucl. Med. Biol. 16, 57-80.

Anke, M., et al., 1971. "Resorption, Exkretion und Verteilung von ${ }^{99}$ Molybdän nach oraler Gabe an Laktierende Wiederkäuer," Arch. Tierernährung, Bd. 21, 505-513.

Antonova M. V., V. A. Pristupa, and V. P. Popov 1968. "Effect of Small Quantities of Microelements in Food Ration on the Immunobiological Reactivity of the Body," Gig. Sanit. (3), 39-42.

Arthur, D. 1965. "Interrelationships of Molybdenum and Copper in the Diet of the Guinea Pig," J. Nutr. 87, 69-76.

Baes, C. F. III, R. D. Sharp, A. L. Sjoreen, and R. W. Shor. 1984. A Review and Analysis of Parameters for Assessing Transport of Environmentally Released Radionuclides Through Agriculture. ORNL-5786. Oak Ridge National Laboratory. 150 pp.

Ballou, J. E. 1960. Metabolism of $W^{185}$ in the Rat, AEC Research and Development Report HW-64112, Hanford Laboratories.

Bartl, M. D. and C. I. H. Lichtenstein 1976. "Tungsten Carbide Pulmonary Fibrosis-A Case Report," Am. Ind. Hyg. Assoc. J. 37, 668-670. 
Bartter, T., et al., 1991. "Zirconium Compound-Induced Pulmonary Fibrosis," Arch. Intern. Med. 151, 1197-1201.

Basta N. T and M. A. Tabatabai 1985. "Determination of Total Potassium, Sodium, Calcium, and Magnesium in Plant Materials by Ion Chromatography," Soil Science Soc. Am. J., Soil Sci. Soc. Am. J. 49 76-81.

Batten, J. J. and B. T. Moore. Corrosion of High-Density Sintered Tungsten Alloys. Part 2: Accelerated Corrosion Testing. Australia Department of Defense, Materials Research Laboratory. Dec. 1988.

Batten, J. J.; I. G. McDonald; B. T. Moore; and V. M. Silva. Corrosion of High-Density Sintered Tungsten Alloys. Part 1: Immersion Testing. Australia Department of Defense, Materials Research Laboratory. October 1988.

Bech, A. O., M. D. Kiplin and J. C. Heather 1962. "Hard Metal Disease," Br. J. Ind. Med. 19, 239-252.

Bech, A. O. 1974. "Hard Metal Disease and Tool Room Grinding," J. Soc. Occup. Med. 24, 11-16.

Belies, R. P. "The Lesser Metals," in Toxicity of Heavy Metals in the Environment, Part 2, ed. F. W. Oehme, Marcel Dekker, Inc., New York 562-564.

Bell, M. C., N. N. Sneed, and R. F. Hall 1966. "Effect of Ration and Route of Administration on Metabolism of ${ }^{99}$ Mo by Sheep and Swine," Proceedings of the 7th International Congress on Nutrition, Vol 5, 765-770.

Bell, M. C. et al., 1964. "Comparison of $\mathrm{Mo}^{99}$ Metabolism in Swine and Cattle as Affected by Stable Molybdenum," J. Nutr. 84, 367-372.

Bell, M. C. and N. N. Sneed 1970. "Metabolism of Tungsten by Sheep and Swine," in Trace Elements Metabolism in Animals, C.F. Mills, Ed., 70-72.

Bell, M. C. et al 1964. "Mo ${ }^{99}$ Metabolism in Swine and Cattle as Affected by Stable Molybdenum," Fed. Proc. Fed. Am. Soc. Exp. Biol. 23, 367-372.

Bentley, M. M., J. H. Williamson and M. J. Oliver 1981. "The Effects of Molybdate, Tungstate and Lxd on Aldehyde Oxidase and Xanthine Dehydrogenase in Drosophila-melanogaster," Canadian J. Genetics Cytol. 23, 597-609.

Berry, J. P., F. Bertrand, and P. Galle 1990. "Subcellular Localization of Zirconium in Nodular Lymphatic Cells after Administration of Soluble Salts." Study by Electron Microprobe, Toxicology 62, 239-246.

Berry, J. P. and P. Galle 1992. "Preferential Localization of Hafnium in Nodular Lymphatic Cells. Study by Electron Microprobe," J. Submicrosc. Cytol. Pathol. 24, 15-18. 
Bibr, B. et al., 1987. "The Mechanism of Action of Molybdenum and Tungsten upon Collagen Structures in vivo," Physiologia Bohemoslovaca 36, 417-424.

Bienvenu, P., C. Nofre, and A. Cier 1963. "Toxicite Generale Comparee des Ions Metalliques. Relation avec la Classification Periodique," Comptes Rendus Hebdomadaires des Seances, Academie des Sciences 256, 1043-1044.

Bischoff, H., et al., 1987. "Efficacy of Beta-Diketonato Complexes of Titanium, Zirconium and Hafnium Against Chemically-Induced Autochthonous Colonic Tumors in Rats," J. Cancer Res. Clin. Oncol. 113, 446-450.

Black, R. G., J. Abraham, and A. A. Ward, Jr. 1967. "The Preparation of Tungstic Acid Gel and Its Use in the Production of Experimental Epilepsy," Epilepsia, 8, 58-63.

Bonde, J. P. 1990. "Semen Quality and Sex Hormones Among Mild Steel and Stainless Steel Welders: A Cross Secitonal Study," Br. J. Ind. Med. 47, 508-514.

Bose, A.; J. Lankford, Jr.; and H. Couque. Development and Characterization of Adiabatic Shear Prone Tungsten Heavy Alloys. July 1993.

Bowers, M.B., E. Goodman, and V. M. Sim 1964. "Some Behavioral Changes in Man Following Anticholinesterase Administration," J. Nerv. Ment. Dis., 138, 383.

Brown, J. R., E. Mastromatteo, and J. Horwood 1963. "Zirconium Lactate and Barium Zirconate: Acute Toxicity and Inhalation Effects in Experimental Animals," Am. Ind. Hyg. Assoc. J. 24, 131-136.

Brown, E.C., Jr. 1948. Effects of G-Agents on Man: Clinical Observations. Medical Division Report 158, Edgewood Arsenal, MD.

Browning, Ethel. Toxicity of Industrial Metals. Chapter 42, Tungsten. 1969.

Bruckner, H. C. 1967. "Extrinsic Asthma in Tungsten Carbide Worker," J. Occup. Med. 9, 518-519.

Butler, C. C. and R. N. Kniseley 1973. "Nonferrous Metallurgy-II. Zirconium, Hafnium, Vanadium, Niobium, Tantalum, Chromium, Molybdenum, and Tungsten," Anal. Chem. 45, 129R-150R.

Byme J. J. and S. M. Draper 1994. "Hafnium 1991," Coordination Chem. Rev. 134, Part 1, 171-187.

Cabre, F., et al. 1990. "Occurrence and Comparison of Sulfite Oxidase Activity in Mammalian Tissues,” Biochem. Med. Metabolic Biol. 43, 159-162.

Cantone, M. D. et al., 1993. "Response to a Single Oral Test of Molybdenum Staple Isotopes for Absorption Studies in Humans," Physiol. Meas. 14, 217-225.

Cantone, M. C. et al., 1992. "Molybdenum Metabolism Studied by Means of Stable Tracers," Med. Phys. 19(2), 439-444. 
Capilna, S., et al. 1963. "Effect of Molybdenum and Tungsten Ions on Intermediate Metabolism of Glutamine in Rat Liver and Brain," Nature, 200, 470.

Cardin, C. J. and J. Mason 1976. "Molybdate and Tungstate Transfer by Rat Ileum Competitive Inhibition by Sulphate," Biochim. Biophys. Acta 55, 937-946.

Carlsen, E., et al. 1992. "Evidence for Decreasing Quality of Semen During past 50 Years," $\mathrm{Br}$. Med. J., 305, 609-613.

Casto, B. C., J. Meyers, and J. A. DiPaolo 1979. "Enhancement of Viral Transformation for Evaluation of the Carcinogenic or Mutagenic Potential of Inorganic Metal Salts," Cancer Res. 39, 193-198.

Chakraborty, D. and A. K. Das 1989. "Indirect Determination of Tungstate in Rat Tissues by Atomic Absorption Spectrometry," Analyst 114, 67-69.

Chanh P-H. 1965. "The Comparative Toxicity of Sodium Chromate, Molybdate, Tungstate, Metavanadate. I.-Experiments on Mice and Rats," Arch. int. Pharmacodyn. 154, 243-249.

Chapelle, S. and J. F. Verchere 1991. "A C-13-NMR Study of the Tungstate and Molybdate Complexes of Perseitol, Glactitol, and D-Mannitol," Carbohydrate Res. 211, 279-286.

Chashchina, N. M. and N. N. Lyalikova 1989. "Role of Bacteria in Transformation of Tungsten Minerals," Microbiol. 58, 104-108.

Chatterjee, G. C., et al. 1973. "Effect of Chromium and Tungsten on L-Ascorbic Acid Metabolism in Rats and Chicks," J. Nutr. 103, 509-514.

Chengelis, C. P. 1991. "The Effect of Molybdenum Depletion of Sulfite Oxidase, Cytochrome P-450, and Carbon Disulfide-Induced Hepatotoxicity in Rats," J. Am. College of Toxicol. 10, 487-491.

Chertok, R. J. and S. Lake 1971. "Biological Availability of Radionuclides Produced by the Plowshare Event Schooner-II. Retention and Excretion Rates in Peccaries after a Single Oral Dose of Debris," Health Physics 20, 325-330.

Chertok, R. J. and S. Lake 1971. "Biological Availability of Radionuclides Produced by the Plowshare Event Schooner-I. Body Distribution in Domestic Pigs Exposed in the Field," Health Physics 20, 317-324.

Chertok, R. J. and S. Lake 1971. "Availability in the Peccary Pig of Radionuclides in Nuclear Debris from the Plowshare Excavation Buggy," Health Physics 20, 313-316.

Chiang, G., L. Dulak, and A. F. Gunnison 1981. "The Embryotoxic and Teratological Evaluation of Dietary Sulfite in Sulfite Oxidase Deficient Rats," The Toxicologist, 1, 30.

Chiang, G., L. Dulak, and A. F. Gunnison 1981. "Embryotoxic and Teratological Evaluation of Dietary Sulfite in Sulfite Oxidase Deficient Rats," Toxicologist 1, 30. 
Clayton G. D. and F. E. Clayton 1981. Patty's Industrial Hygiene and Toxicology, 3rd ed., John Wiley \& Sons, New York, 1981-1994.

Clayton G. D. and F. E. Clayton 1981. Patty's Industrial Hygiene and Toxicology, 3rd ed., John Wiley \& Sons, New York, 2049-2060.

Coates, E. O. and J. H. L. Watson 1971. "Diffuse Interstitial Lung Disease in Tungsten Carbide Workers," Ann. Intern. Med. 75, 709-716.

Cochran, K. W., et al., 1950. "Acute Toxicity of Zirconium, Columbium, Strontium, Lanthanum, Cesium, Tantalum and Yttrium," Arch. Ind. Hyg. Occup. Med. 1, 637-650.

Cohen, H. J., J. L. Johnson, and K. V. Rajagopalan 1974. "Molecular Basis of the Biological Function of Molybdenum. Developmental Patterns of Sulfite Oxidase and Xanthine Oxidase in the Rat," Arch. Biochem. Biophy. 164, 440-446.

Cohen, H. J., et al. 1973. "Molecular Basis of the Biological Function of Molybdenum. The Relationship between Sulfite Oxidase and the Acute Toxicity of Bisulfite and $\mathrm{SO}_{2}$," Proc. Nat. Acad. Sci. 70, 3655-3659.

Collet, A., F. et al., 1963. 'Etude au Microscope electroique de Certains Aspects Cellulaires D'une Fibro-Adenomatose Pulmonaire avec Trouble de Diffusion-Discussion du Role Etiologique du Colbalt et du Carbure de Tungsten," Rev. Tuberc. et Pneumol 27(4), 358-381.

Comar, C. L., L. Singer, and G. K. Davis 1949 "Molybdenum Metabolism and Interrelationships with Copper and Phosphorus," J. Biol. Chem. 180, 913-922.

Comar, C. L. 1948. "Radioisotopes in Nutritional Trace Element Studies-1," Nucleonics 32-45.

Coughlan, M. 1980. “Aldehyde Oxidase, Xanthine Oxidase and Xanthine Dehydrogenase; Hydroxylases Containing Molybdenum, Iron-Sulphur and Flavin," in Molybdenum and Molybdenum-Containing Enzymes, ed. M. Coughlan, Pergamon Press, New York 119-185.

Coughtrey, P. J., D. Jackson, and M. C. Thorne 1983. Radionuclide Distribution and Transport in Terrestrial and Aquatic Ecosystems A Critical Review of Data, Vol. 2, A. A. Balkema, Rotterdam, 351-368.

Couture, P., et al., 1989. "Zirconium Toxicity Assessment Using Bacteria, Algae and Fish Assays," Water Air Soil Pollution 47, 87-100.

Craig, B. G.; R. E. Honnell; G. F. Lederman, Jr.; and D. J. Sandstorm. High Density Metal and Metallic Composites for Improved Fragmentation Submunitions. Aug. 1975.

Craig, A.B. and M. Cornblath 1953. Further Cinical Observations in Workers Accidentally Exposed to G-Agents. Medical Laboratory Research Report 234, AD025225, Edgewood Arsenal, MD. 
Daniel J. W. 1986. "Metabolic Aspects of Antioxidants and Preservatives," Xenobiotica 46, 1073-1078.

De Sousa Pereira, A. et al., 1992. "Evidence of Drainage of Tungsten Particles Introduced in the Pleural Space Through the Visceral Pleura into the Lung Parenchyma," Acta Anat. 145, 416419.

De Renzo, E. C. 1954. "Studies on the Nature of the Xanthine Oxidase Factor," Ann. N. Y. Acad. Sci. 57, 905-908.

Delahant, A. B. 1955. "An Experimental Study of the Effects of Rare Metals on Animal Lungs," AMA Arch. Ind. Health 12, 116-120.

Delongeas, J. L., et al., 1983. "Toxicity and Pharmacokinetics of Zirconium Oxychlorure in the Mouse and in the Rat," J. de Pharmacologie 14(4), 437-447.

Dencker, L. and B. R. Danielsson 1987. "Transfer of Drugs to the Embroy and Fetus after Placentation," Pharmacokinetics in Teratogenesis 1, 55-69.

Deng, M. D., T. Moureaux, and M. Caboche 1989. "Tungstate, a Molybdate Analog Inactivating Nitrate Reductase, Deregulates the Expression of the Nitrate Reductase Structural Gene," Plant Physiol. 91, 304-309.

Devyatka, D. G., et al., 1971. "On the Effect of Molybdenum on Immunological Reactivity," Hyg. Sanit. 36, 133-134.

Dhir, H., et al., 1992. "Interaction Between Two Group IV Metals Lead and Zirconium in BoneMarrow Cells of Mus-musculus In Vivo," Biometals 5, 81-86.

Diapaolo, J. A. and B. C. Casto 1979. "Quantitative Studies of in Vitro Morphological Transformation of Syrian Hamster Cells by Inorganic Metal Salts," Cancer Res. 39, 1008-1013.

Dick, A. T. 1956. "Molybdenum and Copper Relationships in Animal Nutrition," in Inorganic Nitrogen Metabolism, 2nd ed., eds. W. D. McElroy and B. Glass, John Hopkins, Baltimore, 445-473.

Draper, S. M. and B. Twamley 1994. "Zirconium 1991," Coordination Chem. Rev. 134, Part 1 189-229.

Durbin, P. W. 1960. "Metabolic Characteristics within A Chemical Family," Health Physics 2, $225-$ 238.

Durbin, P.W.; K. G. Scott and J. G. Hamilton 1957. "The Distribution of Radioisotopes of Some Heavy Metals in the Rat," Univ. Calif. Pub. Pharmacol. 3, 1-34.

Edel, J. et al., 1990. "Trace Metal lung Disease: in vitro Interaction of Hard Metals with Human Lung and Plasma Components," Science Total Environ. 95, 107-117. 
Ekman, L.; H. D. Figueiras; B. E. V. Jones; and S. Myamoto. Metabolism of 181W-Labeled Sodium Tungstate in Goats. Sweden. Nov. 1977.

Epstein, W. L. and J. R. Allen 1964. "Granulomatous Hypersensitivity After Use of ZirconiumContaining Poison Oak Lotions," JAMA 190, 940-942.

Epstein W. L., J. R. Skahen, and H. Krasnobrod 1962. "Granulomatous Hypersensitivity to Zirconium: Localization of Allergen in Tissue and Its Role in Formation of Epitheloid Cells," J. Invest. Dermatol. 38, 223-232.

Essington, E. H., H. Nishita, and A. J. Steen 1965. "Release and Movement of Radionuclides in Soils Contaminated with Fallout Material from an Underground Thermonuclear Detonation," Health Physics 11, 689-698.

Fairhall, L. T. 1945. "Inorganic Industrial Hazards," Physiol. Rev. 25, 182-202.

Fairhall, L. T., R. Keenan, and H. P. Brenton 1949. "Cobalt and the Dust Environment of Cemented Tungsten Carbide Industry," Public Health Rep. 64, 485-490.

Fillat, C., J. E. Rodriguez-Gil, and J. J. Guinovart 1992. "Molybdate and Tungstate Act Like Vanadate on Glucose Metabolism in Isolate Hepatocytes," Biochem. J. 282, 659-663.

Fleshman, D., S. Krotz and A. Silva 1966. "The Metabolism of Elements of High Atomic Number," UCRL 14739, 69-86.

Fredrick, W. G. and W. R. Bradley 1946. "Toxicity of Tungsten Carbide," Ind. Med. 15, 482-483.

Friberg, L. and J. Lener 1986. "Molybdenum," in Handbook on the Toxicology of Metals, 2nd ed. eds. L. Friberg, G. F. Nordberg, and V. Houk, Elseiver/North Holland Biochemical Press, New York, 446-461.

Frosch, P. J. and A. M. Klingman 1977. "The Chamber-Scarification Test for Assessing Irritancy of Topically Applied Substances," in Cutaneous Toxicity, Proceedings of the 3rd Conference, eds. V. A. Drill and P. Lazar, Academic Press Inc., New York, 127-154.

Fu, M. H. and M. A. Tabatabai 1988. "Tungsten Content of Soils, Plants, and Sewage Sludges in Iowa," J. Environ. Qual 17, 146-148.

Fu, M. H. and M. A. Tahatahai. 1988. "Tungsten content of soils, plants, and sewages sludges in Iowa." J. Environ. Qual. 17:147-148.

Furr, A. K., W. C. Kelly, C. A. Bache, W. H. Gutenmann, and D. J. Lisk. 1976. "Multielement uptake by vegetables and millet grown in pots on fly ash amended soil." J. Agric. Food Chem. 24(4):885-888.

Furr, A. K., T. F. Parkinson, R. A. Hinrichs, D. R. Van Campen, C. A. Bache, W. H. Gutenmann, L. E. St. John, Jr., I. S. Pakkala, and D. J. Lisk. 1977. "National survey of elements and 
radioactivity in fly ashes-absorption of elements by cabbage grown in fly ash-amended soil mixtures." Environ. Sci. Technol. 11(13):1194-1201.

Furr, A. K., T. F. Parkinson, C. L. Heffron, J. T. Reid, W. M. Haschek, W. H. Gutenmann, C. A. Bache, L. E. St. John, Jr., and D. J. Lisk. 1978. "Elemental content of tissues and excreta of lambs, goats, and kids fed white sweet clover growing on fly ash." J. Agric. Food Chem. 26(4):847-851.

Galle P., J. P. Berry, and C. Galle 1992. "Role of Alveolar Macrophages in Precipitation on Mineral Elements Inhaled as Soluble Aerosols," Environ. Health Perspect. 97, 145-147.

Ghosh, A. K., et al., 1991. "Comparative Efficacy of Chlorophyllin in Reducing Cytotoxicity of Some Heavy-Metals," Biol. Metals 4 158-161.

Ghosh, S., A. Sharma, and G. Talukder 1990. "Cytotoxic Effects of Zirconium Oxychloride on Bone Marrow Cells of Mice," Mutation Res. 243, 29-33.

Ghosh, S. G. Talukder, and A. Sharma 1991. "Cytogenic Effects of Exposure to Zirconium Oxychloride in Human-Leukocyte Cultures," Toxicol. in Vitro 5, 295-299.

Ghosh, S. G. Talukder, and A. Sharma 1992. "Chromosomal Alterations and Sister Chromatid Exchanges Induced by Zirconium Oxychloride in Human Lymphocytes in Vitro with Relation to Age of Donors," Mechanisms Ageing Develop. 62, 245-254.

Ghosh, S., A. Sharma, and G. Talukder 1992. "Zirconium-An Abnormal Trace-Element in Biology," Biological Trace Element Res. 35, 247-271.

Ghosh, S., A. Sharma, and G. Talukder 1991. "Relationship of Clastogenic Effects of Zirconium Oxychloride to Dose and Duration of Exposure in Bone Marrow Cells of Mice in Vivo," Toxicol. Lett. 55, 195-201.

Giordano, G., et al., 1982. "Effects of Tungstate and Molybdate on the Invitro Reconstitution of Nitrate Reductase in Escherichia-coli K-12," FEMS Microbiol. Lett. 13, 317-323.

Girio, F. M., M. T. Amaral-Collaco, and M. M. Attwood 1994. "The Effect of Molybdate and Tungstate Ions on the Metabolic Rates and Enzyme Activities in Methanol-Grown Methylobacterium sp. RXM," Appl. Microbiol. Biotechnol. 40, 898-903.

Gol'dman E. I., N. V. Mezentseva, and O. Ya. Mogilevskaya 1967. "Industrial Dust of Luminophores," in Toxicology of Rare Metals, ed. Z. I. Izrael'son, Israel Program for Scientific Ltd., Jerusalem, 170-181.

Gosselin, R. E., R. P. Smith, and H. C. Hodge 1984. Clinical Toxicology of Commercial Products, 5th ed. Williams \& Wilkins, Baltimore, II-148-149.

Goto, Y., et al. 1992. "Synergism in Insulin-Like Effects of Molybdate Plus $\mathrm{H}_{2} \mathrm{O}_{2}$ or Tungstate Plus $\mathrm{H}_{2} \mathrm{O}_{2}$ on Glucose Transport by Isolated Rat Adipocytes," Biochem. Pharmacol. 44, 174-177. 
Grande, N. R., et al., 1990. "Time Course and Distribution of Tungsten-Laden Macrophages in the Hilar Lymph Nodes of the Dog Lung after Experimental Instillation of Calcium Tungstate into the Left Apical Bronchus," Lymphology 23, 171-182.

Gunnison, A. F., et al. 1987. "Distribution, Metabolism and Toxicity of Inhaled Sulfur Dioxide and Endogenously Generated Sulfite in the Respiratory Tract of Normal and Sulfite OxidaseDeficient Rats," J. Toxicol. Environ. Health, 21, 141-162.

Gunnison, A. F., T. J. Farruggella, et al. 1981. "A Sulphite-Oxidase-Deficient Rat Model: Metabolic Characterization," Fd. Cosmet. Toxicol. 19, 209-220.

Gunnison, A. F., et al. 1988. "The Effect of Inhaled Sulfur Dioxide and Systemic Sulfite on the Induction of Lung Carcinoma in Rats by Benzo[a]pyrene," Environ. Res. 46, 59-73.

Gunnison, A. F., L. Dulak, et al. 1981. "A Sulphite-Oxidase-Deficient Rat Model: Subchronic Toxicology," Fd. Cosmet. Toxicol. 19, 221-232.

Gunnison A. F. 1981. "Sulfite Toxicity: A Critical Review of Invitro and Invivo Data," Fd. Cosmet. Toxicol. 19, 667-682.

Halberg L., B. Sandstrom, and P. J. Aggett 1993. "Iron, zinc and Other Trace Elements," in Human Nutrition and Dietetics, eds. J. S. Garrow and W. P. T. James Churchill Livingstone, London, 201.

Haley, T. J., N. Komesu, and J. Raymond 1960. "Toxicity of Hafnyl Chloride," Fed. Proc. Fed. Am. Soc. 19, 389.

Haley, T. J., R. N. Komesu, and H. C. Upham 1962. "The Toxicologic and Pharmacologic Effects of Hafnium Salts," Toxicol. Appl. Pharmacol. 4, 238-246.

Hartung, M. and H. Valentin 1983. "Lung Fibrosis Caused by Inhalation of Hardmetal-Dust," Zbl. Bakt. Hyg., l. Abt. Org. B 177, 237-250.

Hecht, A. and J. Willis 1983. "Sulfites: Preservatives That Can Go Wrong," FDA Consumer September, 11.

Heck, J. D. and M. Costa 1982. "In Vitro Assessment of the Toxicity of Metal Compounds. I. Mammalian Cell Transformation," Biological Trace Elem. Res. 4, 71-82.

Heidelberg C., et al., 1983. "Cell Transformation by Chemical Agents-A Review and Analysis of the Literature. A Report of the U. S. Environmental Protection Agency Gene-Tox Program," Mutat. Res. 114, 283-385.

Higgins E. S., D. A. Richert, and W. W. Westerfield 1956b. "Molybdenum Deficiency and Tungstate Inhibition Studies," J. Nutr. 59, 539-559. 
Higgins E. S., D. A. Richert, and W. W. Westerfield 1956a. "Competitive Role of Tungsten in Molybdenum Nutrition," Fed. Proc. 15, 274-275.

Hogan, K. G. and A. J. Hutchinson 1965. "Molybdenum and Sulphate in the Diet and the Effect on the Molybdenum Content of the Milk of Grazing Sheep," J. Agric. Res. 8, 625-629.

Huber, C., J. et al., 1994. "Further Characterization of Two Different, Reversible Aldehyde Oxidoreductases from Clostridium formicoaceticum, One Containing Tungsten and the Other Molybdenum," Arch. Microbiol. 162, 303-309.

Hutchison, K. A., et al 1992. "The Protein-Protein Complex between pp60 and hsp90 Is Stabilized by Molybdate, Vanadate, Tungstate, and an Endogenous Cytosolic Metal," J. Biolog. Chem., 267, 13952-13957.

Hwang, P. L. and R. J. Ryan. 1981. "Tungstate Stimulates Adehylate Cyclase." Endocrinology. Vol. 108. p. $435-439$.

Hwang, P. L. and R. J. Ryan 1981. "Tungstate Stimulates Adenylate Cyclase," Endocrinology, 108, 435-439.

Idiatullina, F. K. 1981. "Data Toward Hygienic Normalization of Tungsten in Atmospheric Air (Rus.)," Gig. Sanit., 46(9), 79-81.

Ivengar, V and J. Woittiez 1988. "Trace Elements in Human Clinical Specimens: Evaluation of Literature Data to Identify Reference Values," Clin. Chem. 34(3), 474-481.

Iyengar, G. V., M. E. Kollmer, and H. J. M. Bowen 1978. The Elemental Composition of Human Tissues and Body Fluids, Verlag Chemie, New York.

Iyengar, G. V. 1987. "Reference Values for the Concentrations of As, Cd, Co, Cr. Cu, Fe, I, $\mathrm{Hg}, \mathrm{Mn}$, $\mathrm{Mo}, \mathrm{Ni}, \mathrm{Pb}, \mathrm{Se}$, and $\mathrm{Zn}$ in Selected Human Tissues and Body Fluids," Biol. Trace Elem. Res. 12, 263-295.

Jelmert, O., I. L. Hansteen, and S. Langard 1995. "Cytogenetic Studies of Stainless Steel Welders Using the Tungsten Inert Gas and Metal Inert Gas Methods for Welding," Mutat. Res. 342, $77-85$.

Johnson J. L., et al. 1974. "Molecular Basis of the Biological Function of Molybdenum: MolybdenumFree Xanthine Oxdiase from Livers of Tungsten-Treated Rats," J. Biol. Chem. 249, 5056-5061.

Johnson, J. L. and K. V. Rajagopalan 1976a. "Human Sulfite Oxidase Deficiency. Characterization of the Molecular Defect in a Multicomponent System," J. Clin. Investig., 58, 551-556.

Johnson J. L. and K. V. Rajagopalan 1974. "Molecular Basis for the Biological Function of Molybdenum: Effect of Tungsten on Xanthine Oxidase and Sulfite Oxidase in the Rat," J. Biol. Chem. 249, 859-866. 
Johnson, J. L., H. J. Cohen, and K. V. Rajagopalan 1974. "Molecular Basis of the Biological Function of Molybdenum: Molybdenum-Free Sulfite Oxidase from Livers of Tungsten-Treated Rats," $J$. Biol. Chem. 249, 5046-5055.

Johnson J. L. and K. V. Rajagopalan 1976b. "Purification and Properties of Sulfite Oxidase from Human Liver," J. Clin. Investig. 58, 543-550.

Jordan, C., et al. 1990. "Memory Deficits in Workers Suffering from Hard Metal Disease," Toxicol. Lett., 54, 241-243.

Juhl, John H. Diseases of Occupational, Chemical, and Physical Origin. 1987.

June 1994. "Health and Environmental Consequences of Depleted Uranium Used by the U.S. Army," Summary Report to Congress, U.S. Army Environmental Policy Institute.

Juszczak, A., A. Shigetoshi, and M. W. W. Adams 1991. "The Extremely Thermophilic Eubacterium, Thermotoga Maritima, Contains a Novel Iron-Hydrogenase Whose Cellular Activity Is Dependent upon Tungsten," J. Biol. Chem. 266(21), 13834-13841.

Kalistratova, V. S. and Y. I. Moskalev 1969. "Effect of Different Paths of Administration on the Behavior of Molybdenum-99 in the Body of Rats," in Radioaktivnye Izotopy I Organizm (Radioactive Isotopes and the Body), ACE-tr-7195, ed. Y. I. Moskalev, Izdatel'stov Meditsina, Moscow, 186-191.

Kamboj, V. P. and A. B. Kar 1964. “Antitesticular Effect of Metallic and Rare Earth Salts," J. Reprod. Fertil. 7, 21-28.

Kaplun, Z. S. and N. V. Mezentseva 1959. "Hygienic Evaluation of Aerosols Formed in the Manufacture of Hard Alloy (Rus)," Gig. Sanit., 24, 16-22.

Karantassis, M. T. 1924. "On the Toxicity of Compounds of Tungsten and Molybdenum (Sur la toxicite de composes du tungstene et du molybdene)," Ann. Med. Leg., 5, 44-50.

Karaskova, A., J. Lener and B. Bibr 1985. "Effect of Molybdenum and Tungsten on Blood Glucose and Liver Glycogen in Rats," Physiologia Bohemoslovaca 34, 431.

Kawada, J., et al. 1982. "Thyroid Xanthine Oxidase and Its Role in Thyroid Iodine Metabolism in the Rat: Difference between Effects of Allopurinol and Tungstate," J. Endocr. 95, 117-124.

Kaye, S. V. and D. A. Crossley, Jr. 1968. "Radiotungsten Retention by Two Insect Species," Health Physics 14, 162-165.

Kaye, Stephen V. "Distribution and Retention of Orally Administered Radiotungsten in the Rat." March 1968.

Kaye, S. V. 1968. "Distribution and Retention of Orally Administered Radiotungsten in the Rat," Health Physics 15, 399-417. 
Kazantzis, G. 1986. "Tungsten," in Handbook on the Toxicology of Metals, 2nd ed. eds. L. Friberg, G. F. Nordberg, and V. Houk, Elseiver/North Holland Biochemical Press, New York, 610-622.

Kazanzis, G. 1979. "Tungsten," in Handbook on the Toxicology of Metals, eds., L. Friberg, G. F. Nordberg, and V. B. Vouk, Elsevier/North-Holland Biomedical Press, New York, 637-646.

Keen, C. E., et al., 1992. "Histopathological and Microanalytical Study of Zirconium Dioxide and Barium Sulphate in Bone Cement," J. Clin. Pathol. 45, 984-989.

Khan, M. Z.; J. H. Underwood; I. A. Burch. Stress-Corrosion Cracking of Liquid-Phase Sintered Tungsten Alloys. U.S. Army Armament Research, Development and Engineering Center. October 1988.

Kilbane, J. J. "A Biosystem for Removal of Metal Ions from Water" Gas, Oil, Coal, and Environmental Biotechnology III. 1990. pp. 207-226.

Kinard F. W. and J. Van de Erve 1943. "Effect of Tungsten Metal Diets in the Rat," J. Lab. Clin. Med. 28, 1541-1543.

Kinard, F. W. and J. Van de Erve 1941. "The Toxicity of Orally Ingested Tungsten Compounds in the Rat," J. Pharmacol. Exp. Ther. 72, 196-201.

Kinard F. W. and J. Van de Erve 1940. "Rat Mortality Following Sodium Tungstate Injection," Amer. J. Med. Sci. 199, 668-670.

Kinard, F. W. and J. C. Aull 1945. "Distribution of Tungsten in the Rat Following Ingestion of Tungsten Compounds," J. Pharmacol. Exp. Ther. 83, 53-55.

Knudsen, L. E., et al., 1992. "Biomonitoring of Genotoxic Exposure Among Stainless Steel Welders," Mutat. Res. 279, 129-143.

Kollmer, W. E., P. Schramel, and K. Samsahl 1972. "Simultaneous Determination of Nine Elements in Some Tissues of the Rat Using Neutron Activation Analysis," Phys. Med. Biol. 17, 555-562.

Krackow, E. H. 1956. Toxicology of $V$ agents. CWLR 2065, AD 112236, Chemical Corps Research and Development Command, Chemical Warfare Laboratories, Army Chemical Center, MD.

Kusske, J. A., A. R. Wyler, and A. A. Ward, Jr. 1974. "Tungstic Acid Gel as a Focal Epileptogenic Agent," Exp Neurol, 42, 587-592.

LaBrie, S. T., et al., 1992. "Identification of Two Tungstate-Sensitive Molybdenum Cofactor Mutants, Chl2 and Chl7, of Arabidopsis thaliana," Mol. Gen. Genet. 233, 169-176.

Lamprey, H. and R. L. Ripley 1962. "Ultrafine Tungsten and Molybdenum Powders," J. Electrchem. Soc. 109, 713-716. 
Lasfargues, G., 1992. "Comparative Study of the Acute Lung Toxicity of Pure Cobalt Powder and Cobalt-Tungsten Carbide Mixture in Rat," Toxicol. Appl. Pharmacol. 112, 41-50.

Lechlietner, P., et al., 1993. "Goodpasture's Syndrome. Unusual Presentation after Exposure to Hard Metal Dust," Chest 103, 956-957.

Levan, A. 1956. “Cytological Reactions Induced by Inorganic Salt Solutions,” Nature 156, 751-752.

Levin, H. S., K. R. K. L. Rodnitzky, and D. L. Mick 1976. "Anxiety Associated With Exposure to Organophosphate Compounds," Arch. Gen. Psychiatry, 33, 225-228.

Levy, M. and F. Change. Corrosion Behavior of High Density Tungsten Alloys. 1983.

Lichtenstein, M. E., F. Bartl, and R. T. Pierce 1975., "Control of Colbalt Exposures During Wet Process Tungsten Carbide Grinding," Am. Ind. Hyg. Assoc. J. 36, 879-885.

Liippo, K. K., et al., 1993. "Hypersensitivity Pneumonitis and Exposure to Zirconium Silicate in a Young Ceramic Tile Worker," Am. Rev. Respir. Dis. 148, 1089-1092.

Lison, D. and R. Lauwerys 1993. "Evaluation of the Role of Reactive Oxygen Species in the Interactive Toxicity of Carbide-Cobalt Mixtures on Macrophages in Culture," Arch. Toxicol. 67, 347-351.

Lison, D. and R. Lauwerys 1994. "Cobalt Bioavailability from Hard Metal Particles. Further Evidence that Cobalt Alone Is Not Responsible for the Toxicity of Hard Metal Particles," Arch. Toxicol. 68, 528-531.

Lison, D. and R. Lauwerys 1992. "Study of the Mechanisms Responsible for the Elective Toxicity of Tungsten Carbide Cobalt Powder Toward Macrophages," Toxicology Lett. 60, 203-210.

Lison, D. and R. Lauwerys 1990. "In Vitro Cytotoxic Effects on Co-Containing Dust on Mouse Peritoneal and Rat Alveolar Macrophages," Environm. Res. 52, 187-198.

Lison, D. and R. Lauwerys 1991. "Biological Responses of Isolated Macrophages to Cobalt Metal and Tungsten Carbide Powders," Pharmacol. Toxicol. 69, 282-285.

Liu Y. L. et al., 1986. "Effect of Molybdenum and Tungsten Supplementations on Growth and Plasma Estradiol in Female Rats," Fed. Proc. 45(3), 369.

Luckey, T. D. and B. Venugopal. Metal Toxicity in Mammals. Vol. 1. Physiologic and Chemical Basis for Metal Toxicity. page 184.

Ludwin, Cairelli, and Whalen 1994.

Lundgren, K. D. and A. Swensson 1953. "Experimental Investigations on the Effect Upon Animals of Cemented Tungsten," Acta Medica Scand. 145, fasc. I 20-27. 
Lusky, L. M., H. A. Braun, and E. P. Laug 1949. "The Effect of BAL on Experimental Lead, Tungsten Vanadium, Uranium, Copper and Copper-Arsenic Poisoning," J. Ind. Hyg. Toxicol. 31, 301-305.

L'vov, N. P., K. L. Kalakoutsky, and A. I. Zabolotny 1990. "Proteins of Lupin Seeds Binding Molybdenum, Tungsten and Radionuclides from the Chernobyl Fall-Out," 8th International Congress on Nitrogen Fixation, Routledge, New York, 662.

Maceira, J. M. P., et al., 1984. "Immunohistochemical Demonstration of S-100 Protein AntigenContaining Cells in Beryllium-Induced, Zirconium-Induced and Sarcoidosis Granulomas," J. Clin. Pathol. 81, 563-568.

Malama, Y. G. 1984. "Numerical Modeling of the High-Speed Impact for Different Relationships Between the Impactor and Target Densities," Combustion Explosion Shock Waves 20(4), 465-469.

Mamuro, T.; T. Matsunani; and T. Ishtyama. "Detection of Radiotungsten in Rainwater." Department of Health Physics and Instrumentation, Radiation Center of Osaka Prefature, Osaka, Japan. January 1971.

Markley, B. C. E. Meloan, and J. L. Lambert 1986. "Test Strip for the Rapid Identification of Sulfite on Foods," Analytical Lett. 19, 37-46.

Mason, J., et al., 1989. "Behavior of [185W] Thiotungstates Injected into Sheep and the Influence of Copper: Their Fate and the Effect of the Compounds upon Plasma Copper," J. Inorganic Biochem. 35, 115-126.

McLaughlin, J. K., 1992. “A Nested Case-Control Study of Lung Cancer Among Silica Exposed Workers in China," Br. J. Ind. Med. 49, 167-171.

Metcalf, D.R., and J.H. Holmes 1969. "EEG, Psychological, and Neurological Alterations in Humans with Organophosphorus Exposure," Ann. N.Y. Acad. Sci., 160, 357-365.

Mezentseva, N. V. 1967. “Tungsten," Toxicology of Rare Metals, ed. Z. I. Izrael'son, Israel Program for Scientific Ltd. Jerusalem, 28-35.

Miller, C. W., et al., 1953. "Pneumoconiosis in the Tungsten Carbide Tool Industry," AMA Arch. Ind. Hyg. Occup. Med. 8, 453-465.

Mogilevskaya O. Ya. 1967. “Tungsten,” Toxicology of Rare Metals, ed. Z. I. Izrael'son, Israel Program for Scientific Ltd. Jerusalem, 44-52.

Moudgil, V. K., et al. 1983. "Mechanism of Tungstate Action: Inhibition of ATP Activation of Glucocorticoid Receptor,” Fed. Proc., 42, 1260.

Mudd, S. H., F. Irreverre, and L. Laster 1967. "Sulfite Oxidase Deficiency in Man: Demonstration of the Enzymatic Defect," Science 156, 1599-1602. 
Mukund, S. and M. W. W. Adams 1991. "The Novel Tungsten-Iron-Sulfur Protein of The Hyperthermophilic Archaebacterium, Pyrococcus furiosus, Is An Aldehyde Ferredoxin Oxidoreductase: Evidence for Its Participation in A Unique Glycolytic Pathway," J. Biol. Chem. 266(22), 14208-14216

Mukund, S. and M. W. W. Adams 1990. "Characterization of a Tungsten-Iron-Sulfur Protein Exhibiting Novel Spectroscopic and Redox Properties from the Hyperthermophilic Archaebacterium Pyrococcus furiosus," J. Biol. Chem. 265(20), 11508-11516.

Mukund, S., et al., 1994. "Novel Tungsten-Containing Enzymes from Hyperthermophilic Archaea," FASEB 8, A1365.

Mullen, A. L., E. W. Bretthauer, and R. E. Stanley 1976. “Absorption, Distribution and Milk Secretion of Radionuclides by the Dairy Cow - V. Radiotungsten," Health Physics 31, 417-424.

Munro, N. B., K. R. Ambrose, and A. P. Watson 1994. "Toxicity of the Organophosphate Chemical Warfare Agents GA, GB, and VX: Implications for Public Protection," Environ. Health Perspect., 102, 18-38.

Murakami, N., S. P. Healy, and V. K. Moudgil 1982. "Interaction of Rat Liver Glucocorticoid Receptor with Sodium Tungstate," Biochem. J., 204, 777-786.

Murakami, N., T. M. Quattrociochi, S. P. Healy, et al. 1982. "Effects of Sodium Tungstate on the Nuclear Uptake of Glucocorticoid-Receptor Complex from Rat Liver," Arch. Biochem. Biophys., 214, 326-334.

Murakami, N., J. F. Szocik, et al. 1982. "Interaction of Chick Oviduct Progesterone Receptor with Sodium Tungstate: Inhibition of Activation and DNA Binding," J. Steroid Biochem., 17, 251-260.

Murakami, N., et al., 1982. "Tungstate Inhibition of the Nuclear Uptake of Heat-Activated Rat-Liver Glucocorticoid-Receptor Complex," Fed. Proc. 41, 1243.

Murakami, N., T. M. Quattrociochi, J. F. Szocik, et al. 1982. "pH-Dependent Effects of Sodium Tungstate on the Steroid-Binding Properties of Hen Oviduct Progesterone Receptor," Biochim. Biophys. Acta, 719, 267-272.

Nadeenko, V. G., et al. 1978. "The Influence of Tungsten, Molybdenum, Copper, and Arsenic on the Intrauterine Development of the Fetus (Rus.)," Farmakol. Toksikol., 41, 620-623.

Nadeenko, V. G. and V. G. Lenchenko 1977. "The Nature of the Combined Action of Small Doses of Certain Elements-Antagonists (Rus.)," Gig. Sanit., 8, 30-34.

Nadeenko, V. G., et al. 1977. "New Data for Standardization of Tungsten and Molybdenum in their Separate and Simultaneous Presence in Water Bodies (Rus.)," Gig. Sanit., 3, 7-11. 
Nadeenko, V. G. 1966. "Maximum Permissible Concentrations of Tungsten in Water Basins (Eksperimental'nye dannye $\mathrm{k}$ obosnovaniyu predel'no dopustimoi kontsentratsii vol'frama $\mathrm{v}$ vode vodoemov)," Hyg. Sanitat., 31, 197-204.

National Research Council 1989. "Trace Elements," Recommended Dietary Allowances, 10th ed. National Academy Press, Washington, D.C., 243-246.

National Research Council 1980. "Tungsten," Mineral Tolerance of Domestic Animals, National Academy of Sciences, Washington D.C., 515-524.

Nell, J. A., et al. 1981. "Reproductive Performance of Laying Hens Fed Tungsten," Poultry Sci., 60, 257-258.

Ng, Y. C., C. A. Burton, S. E. Thompson, R. K. Tandy, H. K. Kretner, and M. W. Pratt. 1968. "Prediction of the maximum dosage to man from the fallout of nuclear devices." In Handbook for Estimating the Maximum Internal Dose from Radionuclides Released to the Biosphere. UCRL-50163, Pt. IV. Lawrence Radiation Laboratory.

Ng, Y. C. C. S. Colsher, D. J. Quinn, and S. E. Thompson. 1977. Transfer Coefficients for the Predictions of Dose to Man Via the Forage-Cow-Milk Pathway from Radionuclides Released to the Biosphere. UCRL-51939. Lawrence Livermore Laboratory.

Ng, Y. C., C. S. Colsher, and S. E. Thompson. 1982. Transfer Coefficients for Assessing the Dose from Radionuclides in Meat and Eggs Final Report. NUREG/CR-2876 UCID-19464. Lawrence Livermore National Laboratory.

Nicolaou, G., et al., 1987. "Multielement Determination of Metals in Biological Specimens of Hard Metal Workers: A Study Carried Out by Neutron Activation Analysis," J. Trace Elem. Electrolytes Health Dis. 1, 73-77.

Nielsen, V. G., et al., 1994. "Inactivation of Xanthine-Oxidase with Tungstate Reduces Lung and Liver-Injury After Thoracic Aorta Occlusion and Reperfusion in Rabbits," Gastroenterology 106, A259.

Occupational Safety and Health Administration (OSHA) June 1995. OSHA CD-ROM (OSHA A95-3), Folio Infobase, Dataware Technologies.

Opresko, D. M., S. S. Talmage, and M. W. Daughtery May 1994. "Health and Environmental Data for Metals Being Considered for Use in Ammunition Projectiles," Oak Ridge National Laboratory, Oak Ridge, Tennessee.

Owen, E. C. and R. Proudfoot 1968. "The Effect of Tungstate Ingestion of Xanthine Oxidase in Milk and Liver," Brit. J. Nutr. 22, 331-340.

Pan, Y. W., M. T. Yang, and S. P. Yang 1986. "Effect of Molybdenum and Tungsten Supplementations on Copper-Enzymes of Female Rats Fed AIN-76A or Lab Chow," Fed. Proc. 45, 356. 
Pang, D.; S. C. Fu; G. C. Yang. "Relation Between Exposure to Respirable Silica Dust and Silicosis in a Tungsten Mine in China." 1992. Institute of Occupational Medicine, Beijing, People's Republic of China.

Peao, M. N., A. P. Aguas, and N. R. Grande 1992. "Cellular Kinetics of Inflammation in the Pleural Space of Mice in Response to the Injection of Exogenous Particles," Exp. Lung Res. 18, 863876.

Peao, M. N. et al. 1993. "Inflammatory Response of the Lung to Tungsten Particles: An Experimental Study in Mice Submitted to Intratracheal Instillation of a Calcium Tungstate Powder," Lung 171, $187-201$.

Pereira, A. S. and N. R. Grande 1992. "Particle Clearance from the Canine Pleural Space into Thoracic Lymph Nodes: An Experimental Study," Lympphology 25, 120-128.

Pang, D., S. C. Fu, and G. C. Yang 1992. "Relation Between Exposure to Respirable Silica Dust and Silicosis in a Tungsten Mine in China," Br. J. Ind. Med. 49, 38-40.

Paramus, N. J., et al., July 1990. “Appendix D," in Kinetic Energy Penetrator Environmental and Health Considerations (Danesi Report), U.S. Army Production Base Modernization Activity, Picatinny Arsenal, New Jersey.

Peao, M. N., A. P. Aguas, and N. R. Grande 1992. "Cellular Kinetics of Inflammation in the Pleural Space of Mice in Response to the Injection of Exogenous Particles," Exp. Lung Res. 18, 863-876.

Peão, M. N. D., et al., 1993. "Inflammatory Response of the Lung to Tungsten Particles: An Experimental Study in Mice Submitted to Intratracheal Instillation of A Calcium Tungstate Powder," Lung 171, 187-201.

Price, R. J. and D. N. Skilleter 1986. "Mitogenic Effects of Beryllium and Zirconium Salts on Mouse Splenocytes in Vitro," Toxicol. Lett. 30, 89-95.

Prins, R. A., et al., 1980. "Inhibition of Nitrate Reduction in Rumen Bacteria by Tungstate," Appl. Environ. Microbiol. 40, 163-165.

Quin, B. F. and R. R. Brooks 1972. "The Rapid Determination of Tungsten in Soils, Stream Sediments, Rocks and Vegetation," Anal. Chim. Acta 58, 301-309.

Quinn, B. F. and R. R. Brooks. 1972. "The rapid determination of tungsten in soils, stream sediments, rocks, and vegetation." Anal. Chim. Acta 58:299-309.

Ratto, D., et al., 1988. "Pregnancy in a Woman with Severe Pulmonary Fibrosis Secondary to Hard Metal Disease," Chest 93, 663-665.

Report of Task Group of Committee 2. 1993. "Molybdenum," Vol. 67 of ICRP, Pergamon, Oxford, 45-47. 
Report of Task Group of Committee 2. 1979. "Metabolic Data for Molybdenum. Limits for Intakes of Radionuclides by Workers", Vol 30 of ICRP, Pergamon, Oxford, 83-84.

Riddel, G. D., J. G. Simonson, and H. D. Braymer 1981. "Properties of Variant Nitrogen-Fixing Enzyme from Azotobacter vinelandii Mutant Grown on Tungsten," Biophysical J. 33, A274.

Ringeman, J. K., M. W. Miller, and W. F. Andelt 1993. "Effects of Ingested Tungsten-Bismuth-Tin Shot on Captive Mallard," J. Wildlife Management 57, 725-732.

Rolfe, M. W., et al., 1992. "Hard Metal Pneumoconiosis and the Association of Tumor Necrosis Factor-Alpha," Am. Rev. Respir Dis. 146, 1600-1602.

Rosoff, B. and H. Spencer 1964. "Radiobiology: Fate of Molybdenum-99 in Man," Nature 202, 410411 .

Rosoff, B. and H. Spencer 1973. "The Distribution and Excretion of Molybdenum-99 in Mice," Health Physics 25, 173-175.

Rossman, T. G., et al,, 1991. "Performance of 133 Compounds in the Lambda Prophage Induction Endpoint of the Microscreen Assay and a Comparison with S. typhimurium Mutagenicity and Rodent Carcinogenicity Assays," Mutat. Res. 260, 349-367.

Rossman, T. G., M. Molina, and L. Meyer 1984. "The Genetic Toxicology of Metal Compounds: I. Induction of (lambda) Prophage in E coli WP2s(lambda)," Environ. Mutagen. 6, 59-69.

Rowe, P. B. and J. B. Wyngaarden 1966. "The Mechanism of Dietary Alterations in Rat Hepatic Xanthine Oxidase Levels," J. Biol. Chem. 241, 5571-5576.

Russell, J. A., M. K. Roy and J. C. Sanford 1992. "Physical Trauma and Tungsten Toxicity Reduce the Efficiency of Biolistic Transformation," Plant Physiol. 98, 1050-1056.

S. Adelman Associates. "Contract Summary Report Contract DAAA21-93-D-0001. Task Order No. 0002," Information Search of Toxic-Free Ammunition, U.S. Army ARDEC, Picatinny Arsenal, New Jersey.

Sahle, W. 1992. "Possible Role of Tungsten Oxide Whiskers in Hard-Metal Pneumoconiosis," Chest $102,1310$.

Sahle, W. et al., 1994. "Airborne Tungsten Oxide Whiskers in A Hard-Metal Industry. Preliminary Findings," Ann. Occup Hyg. 38, 37-44.

Sahle, W.; I. Laszlo; S. Krantz; and B. Christensson. Airborne Tungsten Oxide Whiskers in a HardMetal Industry. Preliminary Findings. National Institute of Occupational Health, Aerosol Division, Solna, Sweden. 1993. 
Saracino, L., et al., 1986. " Activation Invitro of Respiratory Nitrate Reductase of Escherchia-Coli-K12 Grown in the Presence of Tungstate-Involvement of Molybdenum Cofactor," European J. Biochem. 158, 483-490.

Savage, J. E. 1968. "Trace Minerals and Avian Reproduction," Fed. Proc. 27, 927-931.

Savage, E.P., et al. 1988. "Chronic Neurological Sequelae of Acute Organophosphate Pesticide Poisoning," Arch. Environ. Health , 43, 38-45.

Schepers, G. W. H. 1955. "The Biological Action of Tungsten Carbide and Cobalt-Studies on Experimental Pulmonary Histopathology," AMA Arch. Ind. Health 12, 140-146.

Schepers, G. W. H 1955. "The Biologic Action of Tungsten Carbide and Carbon-Studies on Experimental Pulmonary Histopathology," AMA Arch. Ind. Health 12, 137-139.

Schepers G. W. H. 1971. "Lung Tumors of Primates and Rodents," Ind. Med. 40, 32-37.

Schepers, G. W. H. 1955. "The Biological Action of Particulate Tungsten Metal-Studies on Experimental Pulmonary Histopathology," AMA Arch. Ind. Health 12, 134-136.

Schroeder, H. A.; and M. Mitchener. 1975. "Life-Term Studies in Rats: Effects of Aluminum, Barium, Beryllium, and Tungsten." The Journal of Nutrition. Vol. 105. p. 421-427.

Schroeder, H. A. and M. Mitchner 1975. "Life-Term Studies in Rats: Effects of Aluminum, Barium, Beryllium, and Tungsten," J. Nutr. 105, 421-427.

Schubert, J. 1947. "Treatment of Plutonium Poisoning by Metal Displacement," Science, 105, 389-340.

Scott, K. G. 1952. “Tracer Studies," UCRL 1694, 7-11.

Scott, K. C. and J. R. Turnlund 1993. "Compartmental Model of Molybdenum (Mo) Metabolism in Adult Men Fed Five Levels of Mo," FASEB J. 7(3), A288.

Seanger, J., et al., 1991. "Tungsten-Enriched Molybdenum-Deficient Diet Protects Against Ischemia Reperfusion Injury in Weanling Rats," Lab. Invest. 64, 40A.

Selle, S. R. 1942. "Effects of Subcutaneous Injections of Sodium Tungstate on the Rat," Fed. Proc. Fed. Am. Soc. Exp. Biol. 1, 165.

Settipane, G. A. 1986. "The Restaurant Syndromes," Arch. Intern. Med. 146, 2129-2130.

Shacklette, H. T., J. A. Erdman, T. F. Harms, and C. S. E. Papp. 1978. "Trace elements in plant foodstuffs." In Dehme, F. W. (ed.) Toxicity of Heavy Metals in the Environment Part 1. Marcel Dekker, Inc., New York. pp. 25-68.

Shacklette, H. T., et al., 1975? "Trace Elements in Plant Foodstuffs," Toxicity of Heavy Metals in the Environment, Part 1, ed. F. W. Oehme, F. W. Marcel Dekker, Inc., New York, 25-68. 
Shalgovana, I. V. 1967. "Hygienic Features of the Production of Rare-Metal Fluorides," Hyg. Sanit. 32(10-12), 343-347.

Sharpe, R. M. and N. E. Skakkebaek 1993. "Are Oesterogens Involved in Falling Sperm Counts and Disorders of The Male Reproductive Tract," Lancet 341, 1392-1395.

Sharpe, R. M. and N. E. Skakkebaek 1993. "Are Estrogens Involved in Falling Sperm Counts and Disorders of the Male Reproductive Tract?," Lancet, 341, 1392-1395.

Shears, G. E., R. J. Neal, and D. A. Ledward 1989. "Effects of Dietary Ion Deficiency and Tungsten Supplementation on ${ }^{59} \mathrm{Fe}$ Absorption and Gastric Retention from ${ }^{59} \mathrm{Fe}$ Compounds in Rats," Brit. J. Nutr. 61, 573-581.

Shelley, W. B. and C. J. Raque 1971. "Experimental Zirconium Granulomas and Chrondromas in CBA Mice," J. Investig. Dermat. 57, 411-417.

Shelley, W. B. 1973. "Chondral Dysplasia Induced by Zirconium and Hafnium," Cancer Res. 33, 287-292.

Shen, T. C., C. S. Ramadoss, and B. Vennesland 1982. "Effect of Reduced Pyridine-Nucleotides and Tungstate on the Invitro Insertion of Molybdenum into Demolybdo-Nitrate Reductase of Chlorella-Vulgaris," Biochimica et Biophysica Acta 704, $227-234$.

Shih, V. E., et al. 1977. "Sulfite Oxidase Deficiency: Biochemical and Clinical Investigations of a Hereditary Metabolic Disorder in Sulfur Metabolism," New England J. Med. 297, 1022-1028.

Sidell, F. R. 1992. "Clinical Considerations In Nerve Agent Intoxication," pp. 155-194 in Chemical Warfare Agents, ed. S. Somani, Academic Press, New York.

Shima, S., et al., 1987. "IgM Antibody Production in Mice Intraperitoneally Injected with Zirconium Oxychloride," Br. J. Ind. Med. 44, 633-637.

Sidell, F. R. and W. A. Groff 1974. "The Reactivatibility of Cholinesterase Inhibited by VX and Sarin in Man," Toxicol. Appl. Pharmacol., 27, 241-252.

Singh, I. 1983. "Induction of Reverse Mutation and Mitotic Gene Conversion by Some Metal Compounds in Saccharomyces cerevisiae," Mutation Res. 117, 149-152.

Sirover, M. A. and L. A. Loeb 1976. "Infidelity of DNA Synthesis Intitro: Screening for Potential Metal Mutagens or Carcinogens," Science 194, 1434-1436.

Sirover, M. A. and L. A. Loeb 1976. "Metal-Induced Infidelity During DNA Synthesis," Proc. Natl. Acad. Sci. 73, 2331-2335.

Sivjakov, K. I. and H. A. Braun 1959. "The Treatment of Acute Selenium, Cadmium, and Tungsten Intoxication in Rats with Calcium Disodium Ethylenediaminetetraactate," Toxicol. Appl.

Pharmacol. 1, 602-608. 
Skeleton, H. G., et al., 1993. "Zirconium Granuloma Resulting from an Aluminum Zirconium Complex-A Previously Unrecognized Agent in the Development of Hypersensitivity Granulomas," J. Am. Acad. Dermatol. 28, 874-876.

Skog, E. 1963. "Skin Affections Caused by Hard Metal Dust," Ind. Med. Surg. 32, 266-268.

Smythe, H. F. Jr., et al., 1969. "Range-Finding Toxicity Data: List VII," Am. Ind. Hyg. Assoc. J. 30, 470-471.

Sora, S., et al., 1986. "Dismoic and Diploid Meiotic Products Induced in Saccharomyces cerevisiae by the Salts of 27 Elements," Mutagenesis 1, 21-28.

Spiethoff, A., et al., 1992. "The Combined and Separate Action of Neutron Radiation and Zirconium Dioxide on the Liver of Rats," Health Phys. 63, 111-118.

Sprince, N. L., et al., 1988. "Cobalt Exposure and Lung Disease in Tungsten Carbide Production," Am. Rev. Respir. Dis., 128, 1220-1226.

Stettler, L. E., D. H. Groth, and S. F. Platek 1983. “Automated Characterization of Particles Extracted from Human Lungs: Three Cases of Tungsten Carbide Exposure," Scanning Electron Microscopy Part I 439-448.

Stradling, G. N., J. W. Stather, and M. R. Bailey 1984. "Lung Clearance Studies on Aerosols Arising During the Production of Uranium-Zirconium Alloys," Intern. J. Rad. Bio. 45, 547.

Tajima, Y., Z. Nagasawa, and J. Tadano 1993. "A Factor Found in Aged Tungstate Solution Enhanced the Antibacterial Effect of Beta-Lactams on Methicillin-Resistant Staphaylococcus aureus," Microbiol. Immunol. 37, 695-703.

Teekel, R. A. and A. B. Watts 1959b. "Tungsten Supplementation of Breeder Hens," Poultry Sci. 38, 791-794.

Teekel, R. A. and A. B. Watts 1959a. "Molybdenum Supplementation of Chick Diets," Poultry Sci. $38,1127-1132$.

Terada, L. S., et al., 1992. "Tungsten Treatment Prevents Tumor Necrosis Factor-Induced Injury of Brain Endothelial-Cells," Inflammation 16, 13-19.

Then, G., et al., 1983. "Hafnium Binding to Rat Serum Transferrin," Hyperfine Interactions 15/16, 893-896.

Then, G. M., et al., 1986. "In Vivo and In Vitro Sutides of Hafnium-Binding to Rat Serum Transferrin," J. Inorg. Biochem. 27, 255-270.

Thompson, K. H. and J. R. Turnlund 1995 "Compartmental Model of Molybdenum Metabolism in Adult Men Fed A Low Molybdenum Diet," FASEB J. 9(4), A1000. 
Tlig, S. 1988. "Fish Debris as Chemical Scavengers of Zirconium and Lanthnum in Ocean Environments- $\mathrm{Zr}$ and Hf Fractionation in Marine Phosphates," Chemical Geol. 69, 59-71.

Turnlund, J. R., et al., 1995. "Molybdenum Absorption, Excretion, and Retention Studied with Stable Isotopes in Young Men During Depletion and Repletion ${ }^{1-4}, "$ Am. J. Clin. Nutr. 61, 1102-1109.

Turnlund, J. R., W. R. Keyes, and G. L. Peiffer 1992. "A Stable Isotope Study of Molybdenum Absorption and Urinary Excretion in Young Men," FASEB J. 6(5), A1946.

Turnlund, J. R., W. R. Keyes, and G. L. Peiffer 1993. "Absorption, Retention, and Excretion of A Stable Isotope of Molybdenum in Young Men During Molybdenum Depletion and Repletion," FASEB J. 7(3), A279.

Twarog, F. J. and D. Y. M. Leung 1982. "Anaphylaxis to a Component of Isoetharine (Sodium Bisulfite," JAMA 248, 2030-2031.

Underwood, E. J. 1971. "Molybdenum," in Trace Elements in Human and Animal Nutrition, 3rd ed., Academic Press, New York, 116-140.

Vengerskaya, K. Y. and S. S. Salikhodzhaev 1962. "Some Problems Relating to the Effects of Tungsten Powder on Humans," Gig. Tr. Prof. Zabol., 6, 27-29.

Venitt, S. and L. S. Levy 1974. "Mutagenicity of Chromates in Bacteria and Its Relevance to Chromate Carcinogenesis," Nature 250, 493-495.

Venugopal B. and T. P. Luckey 1975. "Toxicology of Non-Radioactive Heavy Metals and Their Salts," in Environmental Quality and Safety, Supplement Vol. I, eds. T. D. Luckey, B. Venugopal, and D. Hutcheson, Georg Thieme Publishers, Ttuttgart, 4-73.

Venugopal, B. and T. D. Luckey 1978. Metal Toxicity in Mammals, Vol. 2, Plenum Press, New York, 257-259.

Venugopal, B. and T. D. Luckey 1978. Metal Toxicity in Mammals, Vol. 2, Plenum Press, New York, 198-204.

Vernot, E. H., et al., 1977. "Acute Toxicity and Skin Corrosion Data for Some Organic and Inorganic Compounds and Aqueous Solutions,” Toxicol. Appl. Pharmac. 42, 417-423.

Vinogradov, A. P., E.E. Vainshtein, and L. I. Pavlenko. 1958. "Tungsten and molybdenum in igneous rocks (as related to the geochemistry of tungsten)." Geochemistry 5:497-509.

Voronov, V. P. 1983. "Hygienic Assessment of Tungsten as An Air Pollutant," Gig. Sanit. 48(7) 71-72.

Voroshilin, S. I., et al., 1978. "Cytogenic Action of Inorganic Compounds of Tungsten, Zinc, Cadmium, and Cobalt on Human and Animal Somatic Cells," Cytol. Genetics 12(3), 46-48. 
Wase, A. W. 1956. "Absorption and Distribution of Radio-Tungstate in Bone and Soft Tissues," Archives Biochem. Biophys. 61, 272-277.

Watts, R. et al. 1964. "Enzyme Defect in a Case of Xanthinuria," Nature 201, 395-396.

Wei, H. J., X. M. Dou, and S. P. Yang 1985. "Effects of Molybdenum and Tungsten on Mammary Carcinogenesis in SD Rats," JNCI 74, 469-473.

Wester, P.O. 1973. "Trace Elements in Serum and Urine from Hypertensive Patients Before and During Treatment with Chlorthalidone," Acta Med. Scand. 194, 505-512.

Wester, P.O. 1974. "Trace Element Balances in Relation to Variations in Calcium Intake," Atherosclerosis 20, 207-215.

White, A., P. Handler, and E. L. Smith 1964. Principles of Biochemistry, 3rd Edition, McGraw-Hill Book Company, New York.

White, H. and H. Simon 1992. "The Role of Tungstate and or Molybdate in the Formation of Aldehyde Oxidoructase in Clostridium-Thermoaceticum and Other Acetogens-Immunological Distances of Such Enzymes," Arch. Microbiol. 158(2), 81-84.

White, H., G. et al., 1989. "Carbolic Acid Reductase: A New Tungsten Enzyme Catalyzes the Reduction of Non-Activated Carbolic Acids to Aldehydes," European Biochem. 184, 89-96.

Wide, J.; B. R. G. Danielsson, ; and L. Dencker. "Distribution of Tungstate in Pregnant Mice and Effects on Embryonic Cells in Vitro." Department of Zoology and Toxicology, Uppsala University, Sweden. December 1984.

Wide, Mariann. "Effect of Short-Term Exposure to Five Industrial Metals on the Embryonic and Fetal Development of the Mouse." Department of Zoology, Uppsala University, Sweden. 1984.

Wide, M. 1984. "Effect of Short-Term Exposure to Five Industrial Metals on the Embryonic and Fetal Development of the Mouse," Environ. Res., 33, 47-53.

Wide, M., B. R. G. Danielsson, and L. Dencker 1986. "Distribution of Tungstate in Pregnant Mice and Effects on Embryonic Cells In Vitro," Environ. Res., 40, 487-498.

Widholm, J. M., J. P. Ranch, and K. Wakasa 1983. "Inhibition of Cultured-Cell Growth by Tungstate and Molybdate," Plant Cell Rep. 2, 15-18.

Wieneke, J. 1994. "Nitrate (NO3-)-N-13 Flux Studies and Response to Tungstate Treatments in WildType Barley and in an NR-Deficient Mutant," J. Plant Nutrit. 17, 127-146.

Wilson, D. O. and J. F. Cline. 1966. "Removal of plutonium-239, tungsten-185, and lead-210 from soils." Nature 209:941-942. 
Wittenauer, J. P. and T. G. Nieh. Development of Fine-Grained, Ductile Tungsten Alloys for Armor/Anti-Armor Application. January 1991.

Wolfe, E. A. and T. M. S. Chang 1987. "Orally Ingested Microencapsulated Urease and an Adsorbent, Zirconium-Phosphate, to Remove Urea in Kidney Failure," Internat. J. Artificial Organs 10, 269-274.

Woodward, R. L.; J. M. Yellup; and M. E. de Morton. Development of a Sintered Tungsten Alloy Penetrator. 1983. Australia

Working, P. K. 1977. "Male Reproductive Toxicology: Comparison of the Human to Animal Models," Environ. Health Perspect., 77, 37-44. Sipes, I. G. and A. J. Gandolfi 1991. "Biotransformation of Toxicants," in Casarett and Doull's Toxicology. The Basic Science of Poisons, 4th ed., eds. M. O. Amdur, J. Doull, C. D. Klaassen, Pergamon Press, New York, 100

Yang, M. T., et al., 1986. "Effects of Molybdenum and Tungsten Supplementation on Female Rats Fed AIN-76A or Purina Lab Chow," J. Nutr. 116, R36.

Zeiger, E., et al., Mortelmans 1988. "Salmonella Mutagenicity Tests: IV. Results from the Testing of 300 Chemicals," Environ. Molec. Mutagene. 11 (Suppl. 12) 1-158.

Zellner, G. and J. Winter 1987. "Growth Promoting Effect of Tungsten on Methanogens and Incorporation of Tungsten-185 into Cells," FEMS Microbiology Lett. 40, 81-87.

Zhou, Y. L. and W. Beck 1994. "Metal-Complexes with Biologically Important Ligands. 71. Carbonyl Cyclopentadienyl Iron, Rughenium and Tungsten Complexes of 1-Thioglucose," J. Organometallic Chem. 479, 217-220. 
APPENDIX B

INDUSTRY AND INTERNATIONAL CONTACTS 



\section{APPENDIX B. INDUSTRY AND INTERNATIONAL CONTACTS}

In the course of this initial investigation many contacts were initiated with private industry, U.S. military and civilian personnel, and international researchers in an attempt to located and identify data bases, research completed and underway, and refereed articles related to the health and environmental effects of tungsten.

\section{B.1 International Contacts}

Relevant articles were found from Australia, Japan, Sweden, the United Kingdom, and China. These articles are included in the Bibliography (Appendix A). Repeated efforts were made to contact experts on international research. The following contacts may be useful in future investigations:

- Fred Adler, Chief, International Technology Transfer (ITT) Branch, U.S. Army Research Laboratory, AMSRL-TT-TI, 2800 Powder Mill Road, Adelphi, MD 20783-1197, Phone: (301) 394-1400, Fax: (301) 394-4795, E-mail: fadler@arl.mil

- Ciril Baskovic, State Secretary for Science and Technology, Ministry of Science and Technology of Solvenia, Phone: +386 611323 124, Fax: +386 611324140

- Olg Bodrov, Social Ecological Union of Russia, Molodezhnaya st. 22-87, 188537, Sosnovy Bor, Russia, Phone: +7 (81269) 49481

- Prof. Rado Bohinc, Ph.D., Ministry of Science and Technology of Slovenia, Solvenska 50, 61000L jubljana, Slovenia, Phone: +386 611311 107, Fax: +386 611324140

- De Xian Cao, Cancer Institute and Hospital, Chinese Academy of Medical Sciences, E-mail: CAODX@bepc2.ihep.ac.cn

- Zhi Fang Chai, Division of Nuclear Techniques Application, E-mail: CHAIZF@bepc2.ihep.ac.cn

- Vladimir V. Chuikov, director of Sosnovy Bor's Department of VNIPIET - Design Institute, Email: vk@sbl.spb.su

- Dr. Bob Dodding, Army Research Laboratory (ARL) specialist in international tungsten research, Phone: (617)923-5340

- Du Fei Fang, Department of Nuclear Science, Fudan University, E-mail:

DFANG@bepc2.ihep.ac.cn

- Dr. Rado Genorio, State Secretary for International Cooperation, Ministry of Science and Technology of Solvenia, Phone: +386 611314 044, Fax: +38661 1324140

- Dr. Mikhail F. Jakushev, director of nuclear waste storage in Sosnovy Vor - "Lenspetskombinat Radon," P.O. Box 5, 188537 Sosnovy Vor, Leningrad reg., Russia, Phone and Fax: +7 (81269) 62996

- Prof. Yury V. Kuznetsov, V. G. Khlopin Radium Institute, Roentgen str. 1, 197022 St.-Petersburg, Russia, Fax: +7 (812) 2478095

- Bo Feng Li, Institute of Heavy Ion Physics, Department of Physics, Peking University, E-mail: BOFENG@bepc2.ihep.ac.cn

- Dr. Olli Niemi, Managing Director, Tampere Technology Center Hermia, Kanslerinkatu 14, FIN33720 TAMPERA, FINLAND, Phone: +358 31316 5550, Fax: +358 313165552

- Chun Lin Wang, China Center of Advanced Science and Technology, E-mail: CLW@bepc2.ihep.ac.cn

- Ding Sheng Wang, Institute of Physics, Academia Sinica, E-mail: DSWANG@bepc2.ihep.ac.cn 


\section{B.2 Industry Contacts}

Following a review of progress in June 1995 with Dr. Robert Oswald, U.S. Army Corps of Engineers, the staff followed recommendations and contacted General Electric Corporation to inquire about possible studies and data related to tungsten use and worker exposure. As the attached exhibit illustrates (see attached letter), no information on tungsten worker exposure or other environmental data on tungsten are available from this source. In addition, contacts with Olin Corporation are ongoing and may produce some information about DU test range cleanup. However, this information has not been released and therefore is not available to include in this report.

The persons contacted at General Electric Corporation and Olin Corporation include:

General Electric Corporation (216) 266-2121:

Jay Pomeroy

A.M. Zielinski

Brian Mayes

Teeve Barbella

Olin Corporation (813) 578-8100:

Edward Alber

Dennis Crabtree

Tom Wisley 
August 15, 1995

Charles R. Kerley, Ph.D.

Environmental Analysis and Assessment

Oak Ridge National Laboratory

P.O. Box 2008

Oak Ridge, TN 37831-6205

Dear Dr. Kerley,

I'm responding to your letter to Jay Pomeroy requesting information concerning any health or animal studies databases, etc. that we might have concerning tungsten.

We have not conducted any animal or health studies for tungsten used at GE Lighting facilities. While we have done air monitoring to determine exposures relevant to the various recommended exposure limits, we have not done any epidemiological work, nor have we compiled any reviews of the basic research other than those published by NIOSH.

Please feel free to contact me (216-266-3349) if you would like to discuss this in further detail.

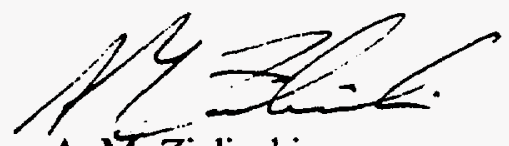

A. M. Zielinski

Lighting Environment, Health and Safety Department 

APPENDIX C

BIOKINETIC MODEL FOR TUNGSTEN

R. W. Leggett 



\section{APPENDIX C. BIOKINETIC MODEL FOR TUNGSTEN}

\section{C.1 INTRODUCTION}

Time-dependent tissue concentrations of a material taken into the human body generally are predicted using a combination of three types of biokinetic models: a respiratory tract model that describes the deposition of inhaled material and subsequent transfer to the gastrointestinal (GI) tract, blood, or the environment; a GI tract model that describes the movement of ingested or endogenously secreted material through the stomach and intestines and transfer to excreta or blood; and a systemic model that describes the time-dependent distribution and excretion of absorbed material. This appendix describes the respiratory, GI, and systemic biokinetic models for tungsten used in this report.

\section{C.2 MODEL OF THE RESPIRATORY TRACT}

Recently the Intemational Commission on Radiological Protection (ICRP) introduced a new respiratory tract model that involves considerably greater detail and physiological realism than previous models of the respiratory system (ICRP 1994). The model structure is shown in Fig. C.1. The model divides the

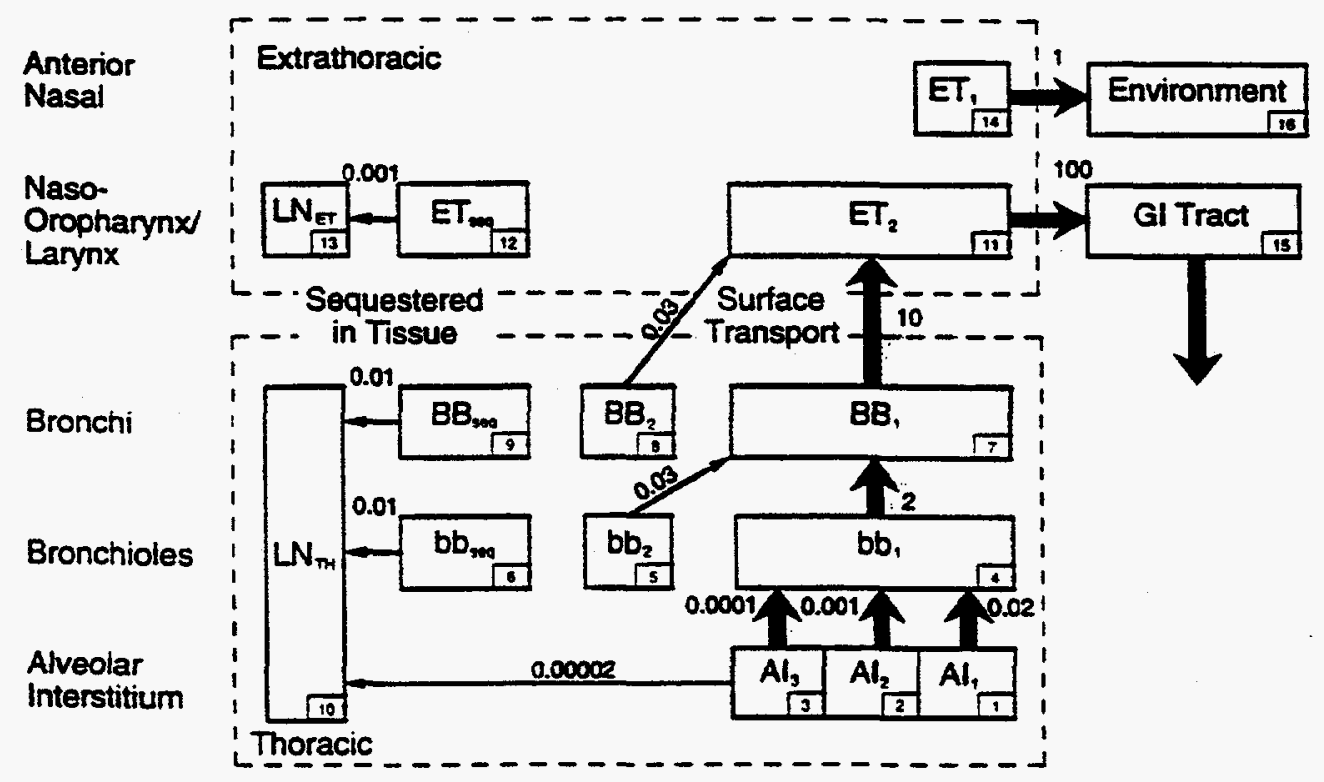

Fig. C.1. Diagram of the respiratory tract model (ICRP 1994).

respiratory system into extrathoracic (ET) and thoracic tissues. The airways of the ET region are further divided into two categories: the anterior nasal passages in which deposits are removed by extrinsic means such as nose blowing, and the posterior nasal passages including the nasopharynx, oropharynx, and the larynx from which deposits are swallowed. The airways of the thorax include the bronchi (BB compartments), bronchioles (bb compartments), and alveolar region (AI compartments). Material deposited in the thoracic airways may be cleared into blood by absorption, to the GI tract by mechanical processes (i.e., transported upward and swallowed), and to the regional lymph nodes via lymphatic channels. 
The number of compartments in each region was chosen to allow duplication of the different kinetic phases observed in experiments on humans and laboratory animals. In Fig. C.1, particle transport rate constants shown beside the arrows are reference values in units of $\mathrm{d}^{-1}$. Compartment numbers shown in the lower right-hand comer of each compartment box are used to define clearance pathways. For example, the particle transport rate from $b_{1}$ to $B B_{1}$ is denoted $m_{4,7}$; transport along this path is assumed to occur at the rate $2 \mathrm{~d}^{-1}$.

For a given inhaled compound, the mechanical clearances of particles indicated in Fig. C.1 are in competition with dissolution rates and absorption to blood, which depend on the element and the chemical and physical form in which it is inhaled. The ICRP plans to develop compound-specific dissolution rates but at present provides only three default solubility types: Type $F$ (fast dissolution and a high level of absorption to blood), Type $M$ (an intermediate rate of dissolution and an intermediate level of absorption to blood), and Type $S$ (slow dissolution and a low level of absorption to blood). The user selects Type F, M, or S based on available experimental data on compounds of the element of interest that are expected to be encountered in practice.

Experimental data on the fate of inhaled tungsten are limited. Best available quantitative information on the deposition and subsequent kinetics of tungsten in the respiratory tract comes from a study on dogs exposed to ${ }^{181} \mathrm{WO}_{3}$ by inhalation (Aamodt 1975). The aerosol had an activity median aerodynamic diameter (AMAD) of $0.7 \mu \mathrm{m}$ with geometric standard deviation of 1.5. Retention and excretion of ${ }^{181} \mathrm{~W}$ were measured over $165 \mathrm{~d}$. An estimated $60 \%$ of inhaled activity was deposited in the respiratory tract and perhaps half of this $60 \%$ was deposited in the lower portion of the tracheobronchial region or the pulmonary region. External measurements over the lungs indicated that roughly $70 \%$ of the deposited material was removed from the lungs with a half-time of $4 \mathrm{~h}, 20-25 \%$ with a half-time of $20 \mathrm{~h}$, and perhaps $5 \%$ with a half-time of $6.3 \mathrm{~d}$. A small percentage may have been retained in the lungs for a period of months. Of the amount leaving the lungs, an estimated 33\% was absorbed directly to blood and the remainder was probably transferred up the tracheobronchial tree and subsequently swallowed. Results of an earlier ingestion experiment by the author indicated that about one-fourth of the swallowed activity may be absorbed from the GI tract to blood.

Grande et al. (1990) studied the time course and distribution of tungsten-laden macrophages in the hilar lymph nodes of the dog lung after experimental instillation of calcium tungstate into the left apical bronchus. They sprayed a tungsten powder $\left(\mathrm{CaWO}_{4}\right)$ into the airway of a single lobe of the dog lung to study the kinetics of particle translocation from the bronchoalveolar lining to hilar lymph nodes and the sorting in lung lymph nodes of inhaled microcrystals. The transport of tungsten particles to the regional lymph node took at least $24 \mathrm{~h}$ and reached its peak at day 7 . Virtually all tungsten in lymph nodes was located in macrophages.

De Sousa Pereira et al. (1992) studied pleural clearance of Ca tungstate particles in the dog by scanning electron microscope and elemental microanalysis. Their results indicate that mesothelial cells are involved in the clearance of particles present in the pleural space. The histological study of lung parenchyma showed many macrophages loaded with tungsten particles.

A large amount of qualitative information but little quantitative data is available on workers exposed by inhalation to various compounds of tungsten. There is evidence that some portion of tungsten inhaled as hard metal dust remains in the lung tissue for an extended time (Sjögren et al. 1980, Cugell et al. 1990, Edel et al. 1990). On the other hand, results of in vitro studies together with measurements 
of tungsten in hair, toenails, and urine of hard metal workers indicate that a substantial portion of tungsten inhaled in the form of hard metal dust may be soluble and highly mobile (Edel et al. 1990).

Peão et al. (1993) used intratracheal instillation of a calcium tungstate powder in mice to study the inflammatory response of the lung to tungsten particles. Tungsten particles were found inside alveolar macrophages throughout the 21-d study.

The above data on inhaled tungsten are not uniformly consistent with any one of the three default solubility types provided in the ICRP's respiratory tract model. Experimental data on dogs (Aamodt 1975, Grande et al. 1990, de Sousa Pereira et al. 1992) seem broadly consistent with Type M. Other data indicate the possibility of long-term retention of a portion of inhaled tungsten in the lungs (Edel et al. 1990, Peão et al. 1993), suggesting that forms of inhaled tungsten may be best represented by Type $S$. In this report, inhaled tungsten is assigned to Type $M$. This was judged to be the more cautious assumption because of the much higher level of absorption to blood associated with Type $M$ than with Type $S$.

\section{C.3 MODEL OF THE GASTROINTESTINAL TRACT}

The model of the GI tract used in this report was developed by Eve (1966) and has been used by the ICRP for several years (ICRP 1979, 1995). The model, shown in Fig. C.2, divides the GI tract into four segments: stomach (S), small intestine (SI), upper large intestine (ULI), and lower large intestine (LLI), and depicts first-order transfer of material from one segment to the next. Material is assumed to transfer from S to SI at the rate $24 \mathrm{~d}^{-1}$, from SI to ULI at $6 \mathrm{~d}^{-1}$, from ULI to LLI at $1.85 \mathrm{~d}^{-1}$, and from LLI to feces at $1 \mathrm{~d}^{-1}$.

Absorption of ingested material to blood is assumed to occur only in the small intestine. Absorption to blood is described in terms of a fraction, $f_{1}$, meaning that the fraction $f_{1}$ of ingested material is absorbed from SI to blood and the fraction 1- $f_{1}$ moves from SI to ULI and eventually is excreted in feces. The ICRP's current $f_{1}$ value for tungsten (ICRP 1981) is 0.3 for all compounds of tungsten other than tungstic acid, for which an $f_{1}$ value of 0.01 is applied (ICRP 1981). As discussed below, the more cautious $f_{1}$ value of 0.5 has been selected for use in this report.

Johnson and coworkers (Johnson and Rajagopalan 1974; Johnson et al. 1974a, 1974b) concluded from studies on rats that the systems for GI absorption and transport of the essential element molybdenum are sufficiently non-specific to accept tungsten instead. Cardin and Mason (1976) found that the maximum rate of transport of $\mathrm{MoO}_{4}{ }^{2-}$ and $\mathrm{WO}_{4}{ }^{2-}$ through the small intestine of the rat, studied in vitro using the everted sac technique, occurs in the lower ileum. Their results indicate that $\mathrm{MoO}_{4}{ }^{2-}, \mathrm{WO}_{4}{ }^{2-}$, and $\mathrm{SO}_{4}{ }^{2-}$ are readily transported across the lower ileum in the rat by a common transport system subject to competitive inhibition. Because the normal dietary level of $\mathrm{SO}_{4}{ }^{2-}$ is orders of magnitude higher than that of $\mathrm{MoO}_{4}{ }^{2-}$ or $\mathrm{WO}_{4}{ }^{2-}$, the extent of absorption of $\mathrm{MoO}_{4}{ }^{2-}$ or $\mathrm{WO}_{4}{ }^{2-}$ may depend on the luminal $\mathrm{SO}_{4}^{2-}$ level. As with molybdenum ingested as molybdate, however, a substantial portion of tungsten ingested as tungstate may be expected to be absorbed to blood under most conditions (Cardin and Mason 1976).

In controlled balance studies lasting five days each, Wester (1974) determined total intake as well as urinary and fecal excretion of tungsten and other nonessential elements in four healthy adult human subjects who were kept on a constant diet. Two studies were performed on each subject, with dietary 


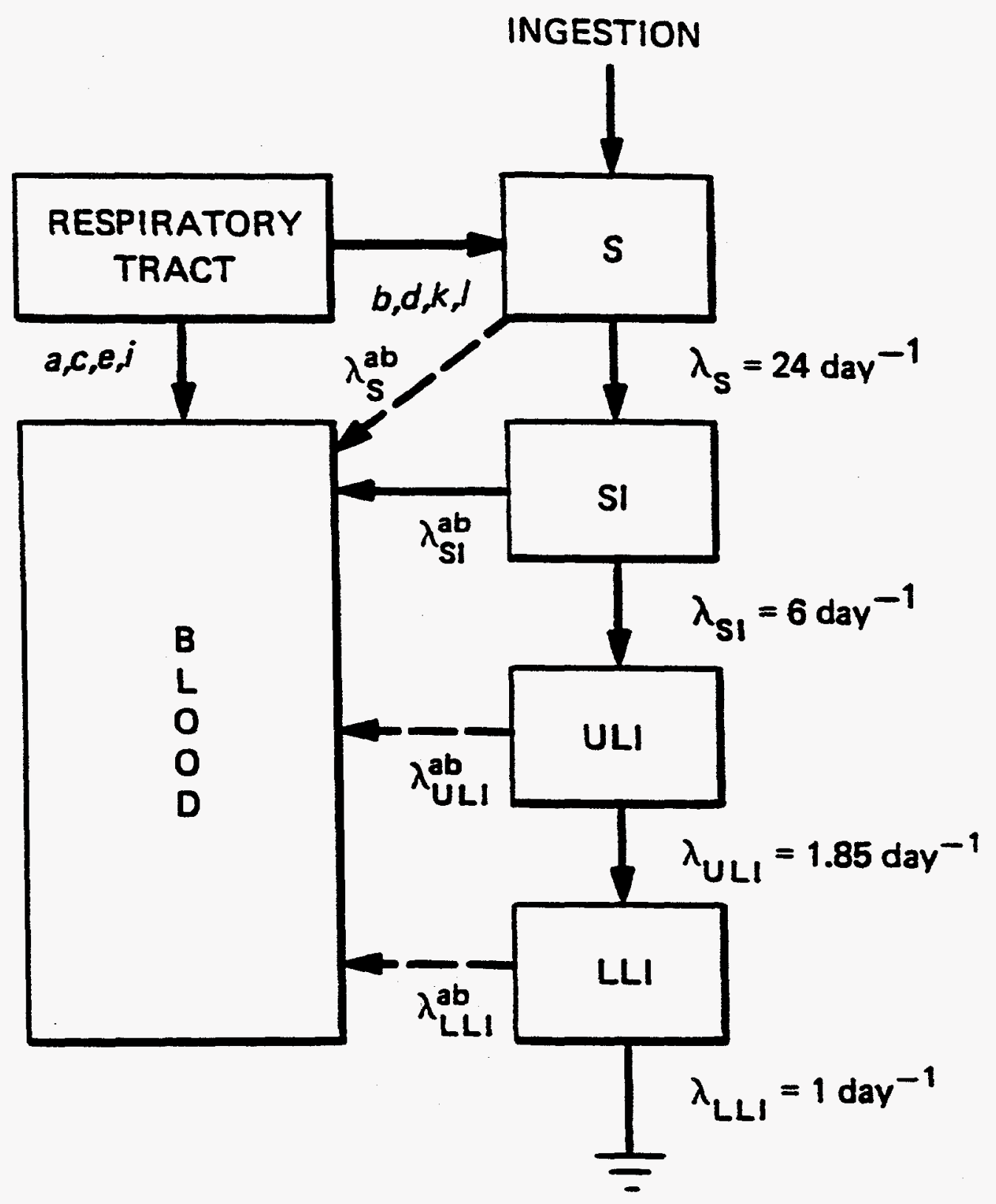

Fig. C.2. Diagram of the gastrointestinal tract model (ICRP 1979).

calcium varied in the two studies. The results of these studies allow a crude estimate of the GI uptake of tungsten, based on the assumptions that the subjects were in tungsten balance, that diet is the primary mode of intake of tungsten, and that daily urinary tungsten approximates daily absorbed 
tungsten. The results of these studies indicate that, as an average, about half of ingested tungsten was absorbed to blood.

Dairy cows absorbed approximately $25 \%$ of tungsten after oral intake of ${ }^{181} \mathrm{~W}$-labeled tungstate (Mullen et al. 1976). A similar level of absorption was determined in a beagle dog after intragastric administration of a weakly acidic aqueous suspension of tungstic oxide (Aamodt 1975). In experiments on rats, GI uptake of radiotungsten administered as tungstate appeared to be in the range $40-70 \%$ (Ballou 1960, Fleshman et al. 1966, Kaye 1968), whereas uptake of radiotungsten administered as tungstic acid was only about $1 \%$ (Ballou 1960). Experiments in which peccaries (pigs) ingested debris from a nuclear explosion indicated GI uptake of 7-20\% (Chertok and Lake 1971a, 1971b, 1971c).

Bell and Sneed (1970) studied the effects of ration and route of administration on the metabolism of ${ }^{185} \mathrm{~W}$ in swine and sheep. Growing swine absorbed at least $75 \%$ of orally administered ${ }^{185} \mathrm{~W}$. Mature sheep fed the same ration absorbed at least $44 \%$ but absorbed more than $65 \%$ of administered ${ }^{185} \mathrm{~W}$ when dosed directly by tube into the abomasum. Gastrointestinal uptake of tungsten was reduced substantially in sheep by diet high in roughage, perhaps due to adsorption of tracer levels of tungsten to feed particles high in cellulose.

In four goats receiving $\mathrm{Na}_{2}{ }^{181} \mathrm{WO}_{4}$ by stomach tube, average absorption of ${ }^{181} \mathrm{~W}$ was only about $5 \%$ of the administered amount (Ekman et al. 1977). The goats' diet was not described in detail but apparently was high in roughage.

The high variability in reported $f_{1}$ values for tungsten is not surprising in view of the apparent dependency of tungsten absorption on the age of the subject and the nature of the diet and its potential dependency on species. Because several of the reported values for laboratory animals fall in the range $40-75 \%$, and because the limited human data suggest a central value around $50 \%$, the $f_{1}$ value of 0.3 used by the ICRP for most compounds of tungsten does not seem adequately cautious for application to human populations. In this report, an $f_{1}$ value of 0.5 is applied to all compounds of tungsten. The case of ingestion of tungstic acid is not considered.

\section{C.4 MODEL OF SYSTEMIC TUNGSTEN}

\section{C.4.1 The Need for a New Systemic Biokinetic Model for Tungsten}

The biokinetic model for tungsten recommended in ICRP Publication 30 (1981) appears to be the most commonly used systemic model for this element. That model (Fig. C.3) was intended to provide reasonable estimates of the integrated activity of radioisotopes of tungsten in different organs, based on biokinetic data for laboratory animals. The model has the limitation that it does not depict biologically realistic paths of movement of tungsten but assumes direct removal of tungsten from tissues to excreta. Such a biologically unrealistic model structure hampers the analysis and unification of the available biokinetic data on tungsten and physiologically related elements. For this reason, a new systemic biokinetic model for tungsten with a more realistic structure was developed for use in this report. 


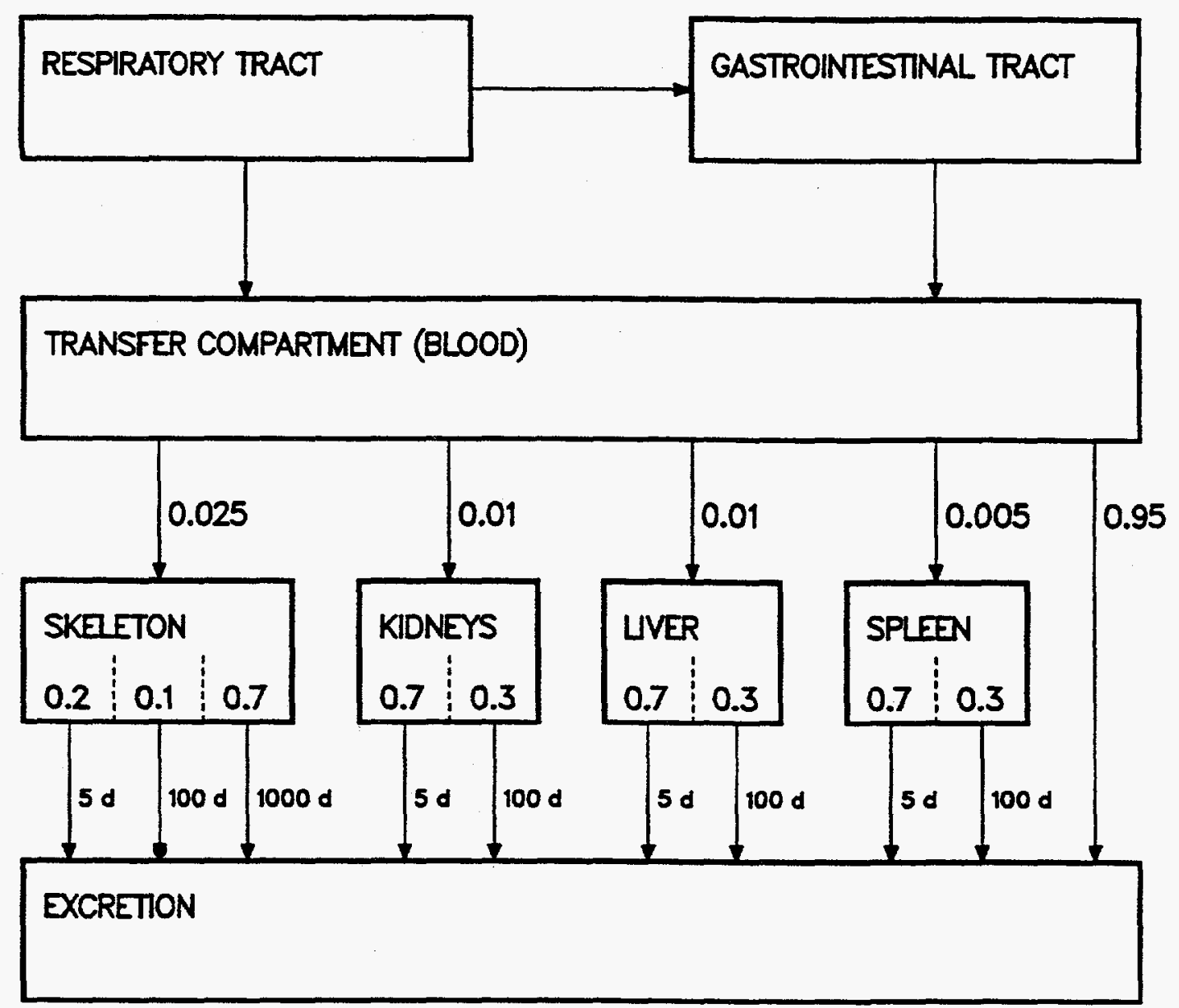

Fig. C.3. Diagram of the biokinetic model for systemic tungsten (ICRP 1981).

\section{C.4.2 Data Used To Develop the Systemic Model for Tungsten}

Information on the fate of absorbed tungsten in humans is limited and consists largely of reported measurements of the concentration of tungsten in tissues, fluids, and excreta of chronically exposed human subjects. These data are too sparse and inconsistent and the patterns of exposure to tungsten are too poorly known to provide much insight into the biokinetics of absorbed tungsten.

The present biokinetic model for systemic tungsten in humans is based on experimental data on the biokinetics of radiotungsten in laboratory animals and information on the kinetics of molybdenum or other physiological analogues of tungsten in humans and laboratory animals. These data are unified within a biologically realistic model structure that incorporates information on the physiological processes that appear to control retention and translocation of heavy metals in the skeleton.

The best available information on the time-dependent distribution and excretion of systemic tungsten in mammalian species comes from studies of: dogs receiving radiotungsten by inhalation or injection (Aamodt 1973, 1975); immature swine exposed to radionuclides produced by a nuclear explosion (Chertok and Lake 1971a, 1971b, 1971c); rodents administered radiotungsten by different routes (Scott 1952, Wase 1956, Ballou 1960, Fleshman et al. 1966, Kaye 1968, Ando et al. 1989); and various farm animals (sheep, pigs, cows, goats) receiving radiotungsten by injection or ingestion (Bell and Sneed 
1970, Mullen et al. 1976, Ekman et al. 1977). The most detailed data are for rats, but it must be considered that rats are often poor laboratory models for man with regard to quantitative biokinetics of absorbed or intravenously injected substances. The most easily interpreted data for larger animals are for dogs (Aamodt 1973, 1975), goats (Ekman et al. 1977), pigs (Bell and Sneed 1970; Chertok and Lake 1971a, 1971b, 1971c), and sheep (Bell and Sneed 1970). Little use was made of data on cows (Mullen et al. 1976) because of difficulties in unfolding biokinetic information from the data and because cows show substantial qualitative differences from more "human-like" animals with regard to the biokinetics of molybdenum, a close physiological analogue of tungsten (Bell et al. 1964, Bell and Sneed 1966). Where species differences in the metabolism of tungsten are indicated, data on dogs, pigs, goats, and sheep were given higher weight in the selection of model parameter values than data on rodents or cows.

Indirect information on the biokinetics of tungsten can also be gained from human and animal data on chemical and physiological analogues of tungsten. For example, there are some similarities in the biological behavior of tungsten and the essential elements molybdenum and phosphorus, probably due to similar tendencies of these elements to form oxyanion compounds in physiological systems. It has been found, for example, that tungstate or molybdate can substitute for phosphate in bone (Fleshman et al. 1966).

Overall, molybdenum appears to be the closest physiological analogue of tungsten. Tungsten often is used as an experimental analogue of molybdenum because it is the element most chemically similar to molybdenum, is the best known biological antagonist of molybdenum, and is the only agent capable of producing experimental molybdenum deficiency in animals by preventing incorporation of molybdenum into certain enzymes (Cardin and Mason 1976). On the other hand, there appear to be some important kinetic differences between tungsten and molybdenum. For example, the liver appears to be an important repository for molybdenum (ICRP 1993) but accumulates a comparatively small fraction of absorbed tungsten (Chertok and Lake 1971a, 1971b, 1971c; Aamodt 1975, Ekman et al. 1977). Another limitation in using molybdenum as a biokinetic surrogate for tungsten is that, despite the considerable attention that has been given to molybdenum metabolism, it has been difficult to determine "typical" biokinetics of molybdenum due to species differences in molybdenum metabolism (Bell et al. 1964, Rosoff and Spencer 1973) and a high variability in molybdenum retention in humans (Turnlund et al. 1995). With regard to development of a biokinetic model for tungsten, data on molybdenum may be most useful as an indicator of the potentially high variability of tungsten metabolism and as a guide to selection of animal models for tungsten metabolism in man.

\section{C.4.3 An Overview of the Systemic Model}

A schematic diagram of the model structure is given in Fig. C.4. The structure is essentially the same as that applied to uranium in ICRP Publication 69 (1995). Although the chemistry and the biological interactions of tungsten differ markedly from those of uranium, the biokinetics of tungsten is similar to that of uranium in the following respects: the main repositories in the body are the same for the two elements; both tungsten and uranium are excreted primarily in urine; most tungsten or uranium reaching blood is excreted in the first $24 \mathrm{~h}$; and at times remote from exposure, most tungsten or uranium in the body is found in the skeleton. Some important differences in the biokinetics of tungsten and uranium are that: tungsten is absorbed to a greater extent than uranium from the GI tract; absorbed tungsten is initially excreted at a faster rate than uranium; and tungsten has lower skeletal uptake than uranium. 


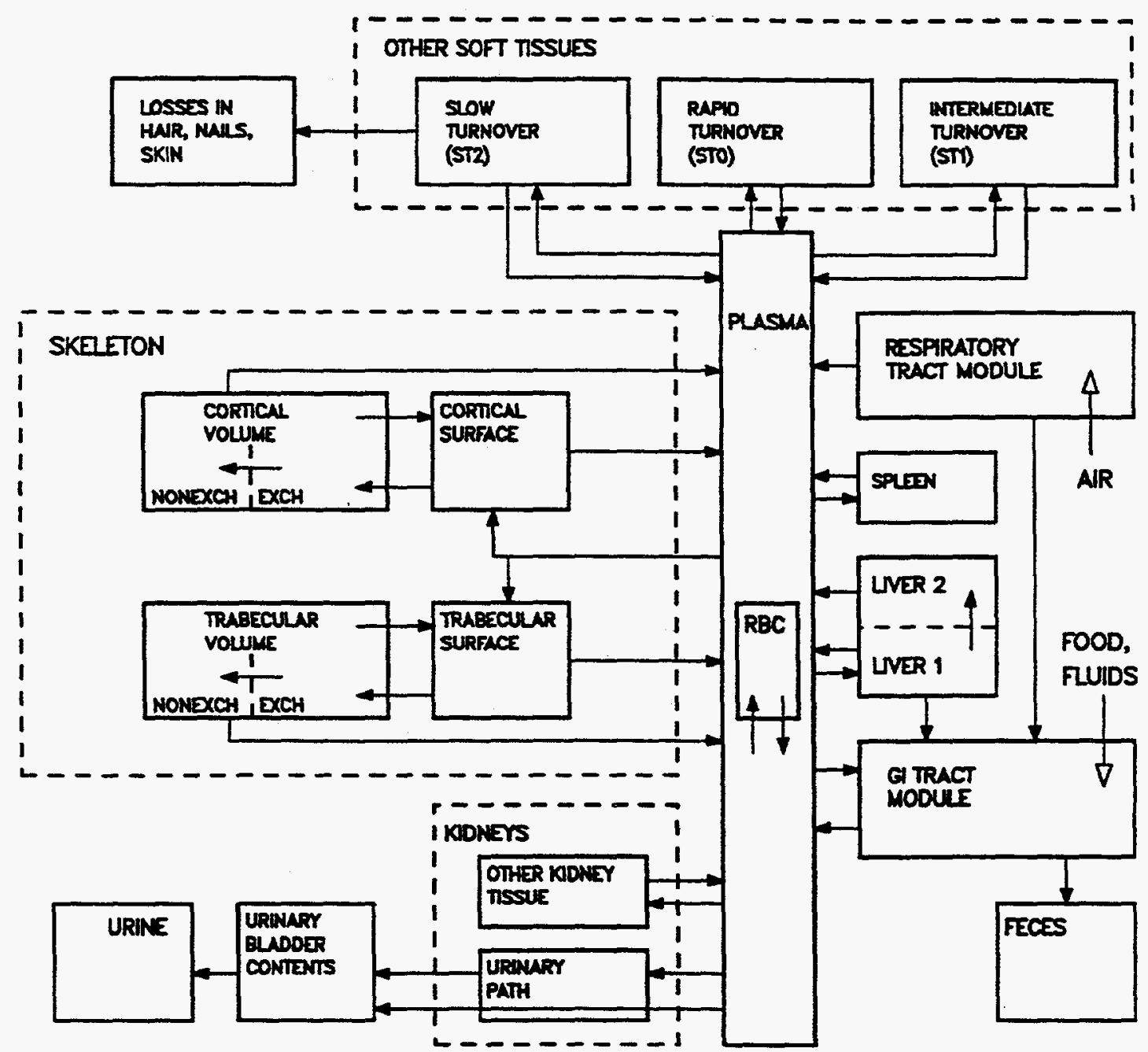

Fig. C.4. Diagram of the biokinetic model for systemic tungsten used in this report.

As in the ICRP's recently adopted model for uranium (ICRP 1995), transport of tungsten between compartments is assumed to follow first-order kinetics. Parameter values are expressed as transfer rates $\left(\mathrm{d}^{-1}\right)$ between compartments because software packages for implementing compartmental models usually require transfer rates as input. The term transfer rate, as used here, indicates fractional transfer per unit time from one compartment to another. The "total transfer rate" from a compartment refers to the sum of transfer rates from that compartment to all destinations. Most of the derived transfer rates in this model are secondary values calculated from selected "removal half-times" and "deposition fractions." The "removal half-time" from a compartment refers here to the biological half-time that one would observe, theoretically, if outflow from that compartment continued while feeds from all other compartments were stopped. The removal half-time as used here generally is shorter than the apparent half-time (i.e., the externally viewed half-time of disappearance) because the latter reflects any feedback of material to a compartment. The "deposition fractions" in this model are used to describe the division of tungsten "leaving the circulation," meaning tungsten that leaves plasma but does not enter the rapidly exchanging extravascular fluids (represented in this model by compartment ST0). Deposition fractions are used mainly to translate experimental data into model transfer rates. These 
fractions are approximately the portions of intravenously injected tungsten that can be found in the compartments at $1 \mathrm{~d}$ after injection.

As discussed later, the total transfer rate from plasma is set at $16.64 \mathrm{~d}^{-1}$, corresponding to a removal half-time of $1 \mathrm{~h}$, and the rapidly exchanging extracellular fluid compartment ST0 is assigned 30\% of instantaneous outflow from plasma. Therefore, the rate at which tungsten leaves the circulation, as defined here, is $0.70 \times 16.64 \mathrm{~d}^{-1}=11.648 \mathrm{~d}^{-1}$. The transfer rate corresponding to a given deposition fraction is calculated relative to the rate of $11.648 \mathrm{~d}^{-1}$ rather than the total transfer rate from plasma of $16.64 \mathrm{~d}^{-1}$. For example, a deposition fraction of 0.04 for the compartment called Liver 1 (Fig. C.4) would mean that this compartment receives $4 \%$ of tungsten leaving the circulation, and the transfer rate from plasma to Liver 1 would be $0.04 \times 11.648 \mathrm{~d}^{-1}=0.46592 \mathrm{~d}^{-1}$. Although the tungsten content of red blood cells (RBC) is actually part of circulating tungsten, it is convenient to assign a deposition fraction to $R B C$ and hence to include the relatively minor uptake of tungsten by $R B C$ as part of tungsten "leaving the circulation."

Transfer rates corresponding to the paths of movement indicated in Fig. C. 4 are summarized in Table 1 . These transfer rates apply to a reference adult. The derivation of these transfer rates is described in the following paragraphs. For expository purposes, the transfer rates are divided into groups describing: (1) circulating tungsten; (2) uptake and retention in the kidneys and urinary excretion; (3) fecal excretion; (4) uptake and retention in the liver; (4) uptake and retention in the spleen; (5) uptake and retention in remaining soft tissues; and (6) uptake and retention in the skeleton.

\section{C.4.4 Transfer Rates Describing Circulating Tungsten}

Data on laboratory animals administered radiotungsten indicate an initially rapid clearance of tungsten from blood but retention of a few tenths of a percent of the administered amount in blood for a period of days (Ballou 1960; Durbin 1957, 1960; Chertok and Lake 1971a, 1971b, 1971c; Aamodt 1973; Mullen et al. 1976; Ekman et al. 1977; Mason et al. 1989; Ando et al. 1989). The high rate of clearance from blood soon after injection may be attributed to a high rate of filtration by the kidneys, together with rapid diffusion from plasma into extravascular fluids. The slower clearance at later times may be due in part to return of tungsten from extravascular spaces to blood and in part to binding to nondiffusible constituents of blood such as RBC and plasma proteins.

After intravenous injection of ${ }^{181} \mathrm{~W}$ as sodium tungstate into beagles, about $70 \%$ of the injected ${ }^{181} \mathrm{~W}$ was removed from blood with a half-time of $35 \mathrm{~min}, 25 \%$ with a half-time of $70 \mathrm{~min}$, and most of the remainder with a half-time of $5 \mathrm{~h}$ (Aamodt 1973). In goats injected intravenously with $\mathrm{Na}_{2}{ }^{181} \mathrm{WO}_{4}$, an estimated $84 \%$ of activity was removed from blood with a half-time of $2 \mathrm{~h}, 15.35 \%$ was removed with a half-time of $9 \mathrm{~h}$, and $0.65 \%$ was removed with a half-time of $63 \mathrm{~h}$ (Ekman et al. 1977). Experiments with cows receiving radiotungsten as tungstate by oral or intravenous administration yielded variable results concerning the residence time of tungsten in blood but generally indicate initially rapid clearance of tungsten from blood and a small component of retention associated with a half-time of several days (Mullen et al. 1976). After intravenous injection of ${ }^{185} \mathrm{~W}$ as tungstate into a sheep, about $25 \%$ of injected activity remained in blood after $0.5 \mathrm{~h}$ and about $10 \%$ remained after $2 \mathrm{~h}$ (Mason et al. 1989). Data on rats (Durbin 1957, 1960; Ballou 1960; Ando et al. 1989) indicate that blood contained substantially less than $1 \%$ of the administered dose of $\mathrm{W}$ at times $\geq 1 \mathrm{~d}$ after exposure. Tissue analysis of young Yorkshire pigs exposed in metabolic cages to radioactive fallout at various distances from a denotation (Chertok and Lake 1971a, 1971b, 1971c) suggest that blood may have contained 10\% or more of total-body radiotungsten at $3 \mathrm{~d}$ after exposure. 
Experimental results concerning the distribution of tungsten between plasma and $R B C$ are variable, perhaps reflecting some species differences in the affinity of tungsten for $\mathrm{RBC}$. In beagles receiving

${ }^{181} \mathrm{~W}$ as sodium tungstate by intravenous injection, the ratio of the concentration of ${ }^{181} \mathrm{~W}$ in plasma to that in RBC averaged about 3 during the first $24 \mathrm{~h}$, and there was no clear trend toward accumulation of activity in RBC during that period (Aamodt 1973). In goats receiving $\mathrm{Na}_{2}{ }^{181} \mathrm{WO}_{4}$ by intravenous administration, steady-state conditions between plasma and RBC were reached at about $6 \mathrm{~h}$ after injection, at which time the RBC contained $10 \%$ of ${ }^{181} \mathrm{~W}$ in blood (Ekman et al. 1977). During multiple oral intakes of ${ }^{181} \mathrm{~W}$ by lactating cows, the concentration of ${ }^{181} \mathrm{~W}$ was higher in whole blood than in serum, and ${ }^{181} \mathrm{~W}$ appeared to have been incorporated into non-serum components of blood shortly after absorption (Mullen et al. 1976). In cows receiving multiple oral administrations of ${ }^{181} \mathrm{~W}$, the concentration of ${ }^{181} \mathrm{~W}$ was about the same in blood as in serum and reached a near steady-state condition during the second day after the beginning of intake. In cows receiving intravenous injections, the concentration of ${ }^{187} \mathrm{~W}$ was about the same in whole blood as in serum and declined with a half-time of about $5 \mathrm{~h}$.

Higher RBC-to-plasma activity ratios for radiotungsten have been determined in rodents than in larger animals. During the period $0.5-72 \mathrm{~h}$ after intragastric administration of ${ }^{187} \mathrm{~W}$ as tungstate to rats, Kaye (1968) observed a gradual accumulation of ${ }^{187} \mathrm{~W}$ in $\mathrm{RBC}$. The concentration of ${ }^{187} \mathrm{~W}$ in $\mathrm{RBC}$ was estimated to be about 9 times higher than that of plasma at $3 \mathrm{~d}$ after administration. In mice, a RBC-to-plasma ratio of about 14 was determined at 3-4 d after intraperitoneal injection of ${ }^{185} \mathrm{~W}$ as tungstate (Wase 1956).

In this model, three compartments are used to describe the kinetics of circulating tungsten: (1) blood plasma, (2) RBC, and (3) a rapid-turnover soft-tissue compartment representing extracellular fluids. The rapid-turnover soft-tissue compartment, called STO, is used to depict an early build-up of tungsten in extravascular spaces followed by relatively rapid feedback to blood.

The total transfer rate from plasma to all destinations is set at $16.64 \mathrm{~d}^{-1}$, corresponding to a removal half-time of $1 \mathrm{~h}$. This is intended as an effective rate over the first few hours after injection and is based mainly on data on dogs (Aamodt 1973) and goats (Ekman et al. 1977). This effective rate probably understates the actual rate of removal from plasma immediately after introduction of tungsten to blood (Aamodt 1973).

Data on uptake and removal of tungsten by soft tissues during the first day after administration are available only for rats (Scott 1952, Ando et al. 1989). Transfer rates for ST0 derived earlier for uranium (Leggett 1994, ICRP 1995) are reasonably consistent with these data and are used in the model for tungsten. Specifically, the rapid-turnover soft-tissue compartment ST0 is assumed to receive $30 \%$ of tungsten leaving plasma and to return tungsten to plasma with a removal half-time of $2 \mathrm{~h}$. The resulting transfer rate from plasma to STO is $0.3 \times 16.64 \mathrm{~d}^{-1}$ or $4.992 \mathrm{~d}^{-1}$, and the transfer rate from ST0 to plasma is $0.693 /(2 \mathrm{~h})=8.32 \mathrm{~d}^{-1}$. The transfer rate from plasma to all destinations other than ST0 (i.e., the rate at which tungsten "leaves the circulation") is $0.7 \times 16.64 \mathrm{~d}^{-1}=11.648$.

Transfer rates for RBC have been set mainly for broad consistency with data on dogs (Aamodt 1973) and goats (Ekman et al. 1977). The RBC compartment is assigned a deposition fraction of 0.005 and a removal half-time of $2 \mathrm{~d}$. The corresponding transfer rate from plasma to $R B C$ is $0.005 \times 11.648 \mathrm{~d}^{-1}=$ $0.05824 \mathrm{~d}^{-1}$. The transfer rate from $R B C$ to plasma is $\ln (2) / 2=0.34657 \mathrm{~d}^{-1}$. 


\section{C.4.5 Transfer Rates Describing Renal Retention and Urinary Excretion}

A substantial portion of tungsten leaving plasma is filtered by the kidneys and ultimately excreted in urine. The rate of urinary excretion may depend on the concentrations of other Group VI oxyanions in the glomerular filtrate (e.g., sulphate and molybdate), because these ions apparently compete for binding sites on a common transport system responsible for uptake in the renal tubular epithelium and reabsorption to blood (Ryan et al. 1987, Mason et al. 1989). In view of the close physiological similarities of tungstate and molybdate, it seems likely that a portion of filtered tungstate is reabsorbed to blood. However, some remains bound for at least a few hours or days in the renal tubules. At $3 \mathrm{~h}$ after intravenous injection of radioisotopes of tungsten and molybdenum into rats, renal retention was considerably greater for tungsten than for molybdenum (Ando et al. 1989).

Experimental data on laboratory animals indicate that a major portion of injected or absorbed tungsten is rapidly excreted in urine. In experiments with rats, urinary excretion accounted for about $75 \%$ of injected or absorbed radiotungsten during the first $3 \mathrm{~h}$ after administration and more than $85 \%$ after 24 h (Durbin 1957, 1960; Fleshman et al. 1966; Kaye 1968; Ando et al. 1989). Goats injected intravenously with ${ }^{181} \mathrm{~W}$-labeled sodium tungstate excreted about $78 \%$ of the administered ${ }^{181} \mathrm{~W}$ in urine during the first day and about $88 \%$ after $8 \mathrm{~d}$ (Ekman et al. 1977). Beagle dogs injected with ${ }^{181} \mathrm{~W}$-labeled sodium tungstate excreted about $91 \%$ of administered ${ }^{181} \mathrm{~W}$ in urine during the first day and excreted a substantial portion of the remaining $9 \%$ in urine over the next few days (Aamodt 1973). Lactating cows injected with ${ }^{187} \mathrm{~W}$ as sodium tungstate excreted about $68 \%$ of administered ${ }^{187} \mathrm{~W}$ in urine during the first $92 \mathrm{~h}$ after administration (Mullen et al. 1976).

A sheep injected intravenously with ${ }^{185} \mathrm{~W}$ as tungstate excreted about $67 \%$ of the dosage in urine in the first $6 \mathrm{~h}$ following administration, more than $90 \%$ in the first $24 \mathrm{~h}$, and virtually $100 \%$ in the first $2 \mathrm{~d}$ (Mason et al. 1989). Considerably lower rates of urinary excretion of ${ }^{185} \mathrm{~W}$ were observed in sheep injected with ${ }^{185} \mathrm{~W}$-trithiotungstate or ${ }^{185} \mathrm{~W}$-tetrathiotungstate (Mason et al. 1989). In animals injected with ${ }^{185} \mathrm{~W}$-trithiotungstate, urinary excretion accounted for roughly half of injected ${ }^{185} \mathrm{~W}$ in the first $2 \mathrm{~d}$ after administration and $70-85 \%$ in the first $5 \mathrm{~d}$ after administration. Animals injected with

${ }^{185} \mathrm{~W}$-tetrathiotungstate excreted less than $25 \%$ of injected ${ }^{185} \mathrm{~W}$ in the first $2 \mathrm{~d}$ and less than $50 \%$ in the first $5 \mathrm{~d}$ after administration.

Data on the retention of tungsten in the kidneys of laboratory animals at times $\geq 3 \mathrm{~h}$ after administration are summarized in Fig. C.5 (Durbin 1960, Ballou 1960, Fleshman et al. 1966, Kaye 1968, Bell and Sneed 1970, Chertok and Lake 1971b, Aamodt 1975, Ekman et al. 1977, Ando et al. 1989). The data indicate that a few percent of filtered tungsten is deposited in the kidneys and that most of the deposited material is removed within several hours or a few days. It appears that a small portion of the deposited tungsten, representing at most a few tenths of a percent of absorbed or injected tungsten, is tenaciously retained in the kidneys of larger animals. Renal retention is considerably lower in rats than in larger animals.

In this model the kidneys are viewed as consisting of two compartments, called "urinary path" and "other kidney tissue." The urinary path compartment is used to depict rapid build-up and decline of filtered tungsten in the kidneys, presumably corresponding to attachment to and loss from the brushborder membranes of epithelial cells of the renal tubules. The other kidney tissue compartment is used to depict long-term retention of tungsten in the kidneys. A portion of tungsten filtered at the glomerulus is assigned to the urinary path compartment and the rest is assigned to bladder urine. Tungsten leaving the urinary path compartment is assigned to bladder urine. The other kidney tissue 


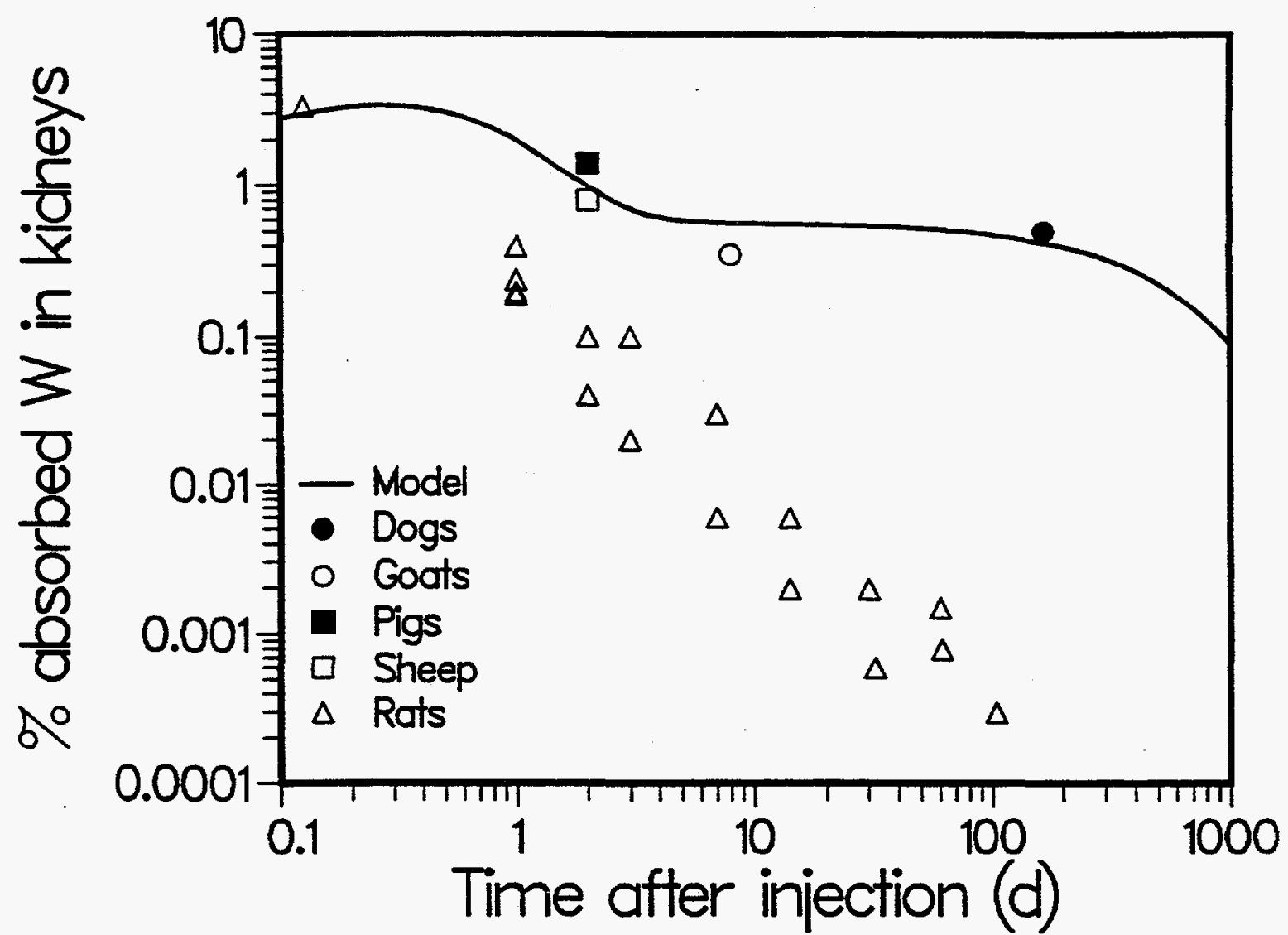

Fig. C.5. Data on retention of tungsten in kidneys of laboratory animals (Durbin 1960, Ballou 1960, Fleshman et al. 1966, Kaye 1968, Bell and Sneed 1970, Chertok and Lake 1971b, Aamodt 1875, Ekman et al. 1977, Ando et al. 1989) and predictions of the present model for the case of intravenous injection of tungsten into a typical adult human.

compartment is assumed to exchange tungsten with the nutritive blood supply, i.e., with the plasma compartment.

It is assumed that $75 \%$ of tungsten leaving the circulation passes directly into the urinary bladder contents and that $5 \%$ deposits in the kidneys. Data on renal uptake and retention of tungsten in larger animals (Fig. C.5) are reproduced reasonably well by assuming that, of the $5 \%$ depositing in the kidneys, $4.5 \%$ deposits in the urinary path compartment and is removed to urine with a half-time of $0.5 \mathrm{~d}$, and the remaining $0.5 \%$ deposits in other kidney tissue and is removed to plasma with a half-time of $1 \mathrm{y}$. Therefore, the transfer rate from plasma to bladder urine is $0.75 \times 11.648 \mathrm{~d}^{-1}=$ $8.7360 \mathrm{~d}^{-1}$, from plasma to the urinary path compartment is $0.045 \times 11.648 \mathrm{~d}^{-1}=0.52416 \mathrm{~d}^{-1}$, from plasma to the other kidney tissue compartment is $0.005 \times 11.648 \mathrm{~d}^{-1}=0.05824 \mathrm{~d}^{-1}$, from the urinary path compartment to urinary bladder contents is $\ln (2) / 0.5=1.3863 \mathrm{~d}^{-1}$, and from the other kidney tissue compartment to plasma is $\ln (2) / 365=0.00190 \mathrm{~d}^{-1}$. 


\section{C.4.6 Transfer Rates Describing Fecal Excretion}

As is the case for molybdenum, endogenous fecal excretion appears to be a relatively minor excretion pathway for injected or absorbed tungsten. For example, the following ratios of urinary to fecal excretion of radiotungsten have been determined in experimental studies on laboratory animals: 13, during the first $2 \mathrm{~d}$ after intraperitoneal injection of ${ }^{187} \mathrm{~W}$ as sodium tungstate into rats (Fleshman et al. $1966) ; 15$, in the first $8 \mathrm{~d}$ after intravenous injection of ${ }^{181} \mathrm{~W}$-labeled sodium tungstate into goats (Ekman et al. 1977); 38, in the first $10 \mathrm{~d}$ after intravenous injection of ${ }^{181} \mathrm{~W}$-labeled sodium tungstate into beagle dogs (Aamodt 1973); and 68, in the first $92 \mathrm{~h}$ after injection of ${ }^{187} \mathrm{~W}$ as sodium tungstate into cows (Mullen et al. 1976). Experimental data for sheep also suggest that fecal excretion is a relatively minor excretion pathway for injected or absorbed tungsten (Bell and Sneed 1970, Mason et al. 1989), although precise urinary-to-fecal excretion ratios cannot be calculated from the reported data.

The pathways by which systemic tungsten is transferred to the intestines have not been determined. Limited data on goats suggest that biliary secretion may not have been the main pathway of endogenous fecal excretion of tungsten in these animals (Ekman et al. 1977).

In this model, a small amount of fecal excretion of tungsten is depicted. For simplicity, it is assumed that there is direct transfer of tungsten from the circulation to the content of the large intestine. Thus, the model depicts only net endogenous fecal excretion of tungsten, because there is likely to be some transfer of systemic tungsten to the small intestine and subsequent reabsorption to blood. Of tungsten leaving the circulation, $5 \%$ is assumed to transfer directly to the contents of the upper large intestine (ULI). Therefore, the transfer rate from plasma to the contents of the ULI is $0.05 \times 11.648 \mathrm{~d}^{-1}=$ $0.5824 \mathrm{~d}^{-1}$.

\section{C.4.7 Transfer Rates Describing Hepatic Retention}

Some uptake and retention of tungsten by the liver may be expected because of the close chemical and physiological resemblance of this element to molybdenum, which is involved in the production of some enzymes in the liver. In particular, molybdenum is a constituent of xanthine oxidase, an enzyme involved in the production of uric acid. In experimental studies on humans as well as laboratory animals, a substantial portion of the total-body content of administered isotopes of molybdenum were found in the liver during the first several days or weeks after intake.

Experimental data on uptake and retention of tungsten in the liver of laboratory animals are summarized in Fig. C.6 (Durbin 1960, Ballou 1960, Fleshman et al. 1966, Kaye 1968, Aamodt 1975, Ekman et al. 1977, Ando et al. 1989). It appears that the concentration of tungsten is higher in the liver than in most other soft tissues but that the liver is a much less important repository for tungsten than for molybdenum.

In this model the liver is viewed as consisting of two compartments, called Liver 1 and Liver 2. Liver 1 is used to describe the short-term kinetics of tungsten in the liver and Liver 2 is used to describe long-term kinetics. Tungsten is assumed to move from plasma into Liver 1. Outflow from Liver 1 is divided between plasma and Liver 2 . Outflow from Liver 2 is assigned to plasma. This qualitative model of the liver has been applied by the ICRP to several nonessential elements and is based on the observation that such elements may enter hepatocytes by the same mechanisms used for essential elements but may later be transferred to, and tenaciously retained in, reticuloendothelial cells of the liver. 


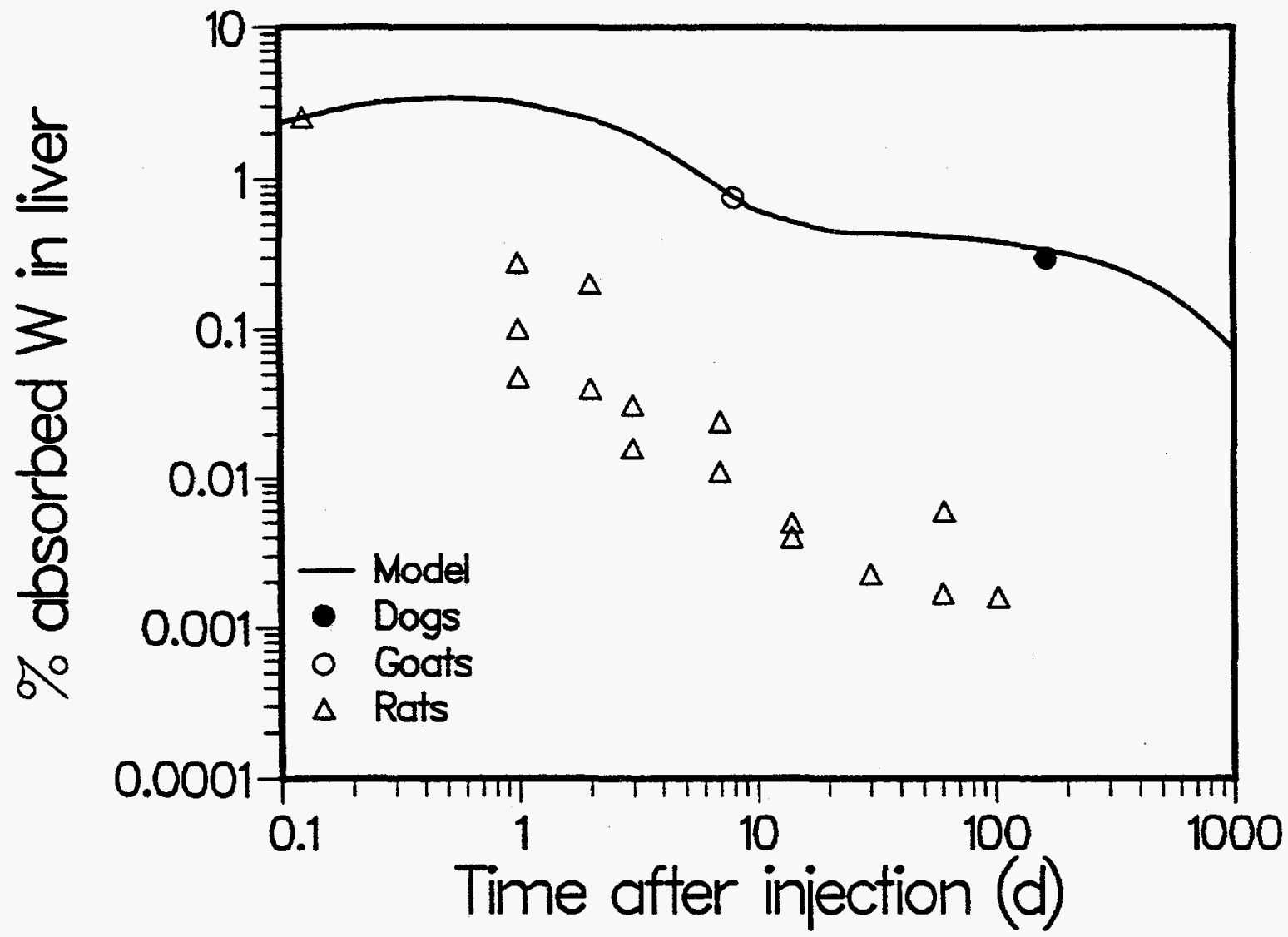

Fig. C.6. Data on retention of tungsten in livers of laboratory animals (Durbin 1960, Ballou 1960, Fleshman et al. 1966, Kaye 1968, Ekman et al. 1977, Ando et al. 1989) and predictions of the present model for the case of intravenous injection of tungsten into a typical adult human.

Experimental data on large laboratory animals exposed to tungsten (Fig. C.6) are reproduced reasonably well by assuming that: $4 \%$ of tungsten leaving the circulation deposits in Liver 1; tungsten is removed from Liver 1 with a half-time of $2 \mathrm{~d}$, with $90 \%$ of outflow retuming to plasma and $10 \%$ depositing in Liver 2; and the removal half-time from Liver 2 is 1 y. The resulting transfer rate from plasma to Liver 1 is $0.04 \times 11.648 \mathrm{~d}^{-1}=0.46592 \mathrm{~d}^{-1}$, from Liver 1 to plasma is $0.9 \times \ln (2) /(2 \mathrm{~d})=$ $0.31192 \mathrm{~d}^{-1}$, from Liver 1 to Liver 2 is $0.1 \times \ln (2) /(2 \mathrm{~d})=0.034657 \mathrm{~d}^{-1}$, and from Liver 2 to plasma is $\ln (2) /(365 d)=0.00190 d^{-1}$.

\section{C.4.8 Transfer Rates Describing Retention in the Spleen}

Experimental data on uptake and retention of tungsten by the spleen are highly variable, perhaps reflecting species differences in the functions of the spleen as well as differences in the chemical and physical properties of administered tungsten. In most studies, however, the concentration of tungsten in spleen at times remote from exposure has been higher than the average for all soft tissues (Ballou 1960; Fleshman 1966; Kaye 1968; Chertok and Lake 1971a, 1971b, 1971c; Kollmer et al. 1972; Aamodt 1975; Mullen et al. 1976; Ekman et al. 1977). Therefore, the spleen is included as a separate compartment in this model. 
Transfer rates for the spleen are based mainly on comparative data for tungsten retention in the spleen and liver of larger laboratory animals. In pigs (Chertok and Lake 1971a, 1971b, 1971c) and goats (Ekman et al. 1977), the concentration of tungsten in spleen was slightly less than that in liver during the first few days after exposure to radiotungstate. In dogs (Aamodt 1975), the concentration of tungsten in spleen was similar to that in liver at 5 mo after inhalation of radiotungstate (Aamodt 1975). In this model it is assumed that the spleen receives $0.05 \%$ of tungsten leaving the circulation and that tungsten is removed from the spleen to blood with a half-time of $1 \mathrm{y}$. Therefore, the transfer rate from plasma to spleen is $0.0005 \times 11.648 \mathrm{~d}^{-1}=0.005824 \mathrm{~d}^{-1}$, and the transfer rate from spleen to plasma is $\ln (2) / 365 \mathrm{~d}=0.00190 \mathrm{~d}^{-1}$. With these assumptions, the predicted concentration of tungsten in the spleen of a reference adult human averages about 0.5 times that in the liver during the first week and slightly exceeds that in the liver after a few weeks.

\section{C.4.9 Transfer Rates Describing Retention in Remaining Soft Tissues}

Limited experimental data on laboratory animals (Ballou 1960; Fleshman 1966; Kaye 1968; Chertok and Lake 1971a, 1971b, 1971c; Kollmer et al. 1972; Aamodt 1975; Mullen et al. 1976; Ekman et al. 1977; Ando et al. 1989) indicate that the pattern of uptake and loss of tungsten by the massive soft tissues (defined here to mean soft tissues other than liver, kidneys, and spleen) is qualitatively similar to that for other metals, including uranium. That is, the massive soft tissues appear to accumulate a substantial portion of systemically deposited tungsten soon after introduction of tungsten to blood but lose most of the initial burden within a period of hours. Thereafter, there is a slower loss of most of the remaining tungsten over a period of days or weeks, and retention of a small fraction of material in some tissue components for a period of months or years. For example, animal data indicate relatively tenacious retention of tungsten in skin and some accumulation in hair (Kaye 1968, Mullen et al. 1976). Also, binding of tungsten and molybdenum to collagen has been observed (Bibr et al. 1987). Limited data on occupationally exposed humans indicate some accumulation of tungsten in nails and hair (Nicolaou et al. 1987).

The soft-tissue compartment STO is used in this model to depict the rapid accumulation and early loss of tungsten in massive soft tissues. Two other soft-tissue compartments, ST1 and ST2, are used to describe intermediate and slow turnover, respectively, in the massive soft tissues.

Transfer rates for ST0 were given earlier. Compartment ST1 is assigned the percentage of tungsten left over after other deposition fractions in the model have been assigned, amounting to $2.25 \%$ of tungsten leaving the circulation. The removal half-time from ST1 to plasma is assumed to be $10 \mathrm{~d}$.

Compartment ST2 is assigned $0.2 \%$ of tungsten leaving the circulation and is assumed to lose this in excreta (in the form of hair, nails, and desquamated skin) with a half-time of 1 year. Therefore, the transfer rate from plasma to ST1 is $0.0225 \times 11.648 \mathrm{~d}^{-1}=0.26208 \mathrm{~d}^{-1}$; from ST1 to plasma is $\ln (2) / 10$ $\mathrm{d}=0.0693 \mathrm{~d}^{-1}$; from plasma to ST2 is $0.002 \times 11.648 \mathrm{~d}^{-1}=0.023296 \mathrm{~d}^{-1}$; and from ST2 to plasma is $\ln (2) / 365 d=0.00190 d^{-1}$.

\section{C.4.10 Transfer Rates Describing Uptake, Translocation, and Retention in the Skeleton}

Results of experimental studies on laboratory animals indicate that a few percent of absorbed tungsten deposits in bone, at least a few tenths of a percent of the absorbed amount is retained for an extended period, and accumulation of tungsten is greater in growing than in mature bone (Fleshman et al. 1966, Kaye 1968, Aamodt 1975, Mullen et al. 1976, Ando et al. 1989). Similarities in the behavior of tungstate, molybdate, and phosphate in biological systems have been observed, and it seems likely that 
uptake and retention of tungsten by bone are due to substitution of tungstate for phosphate (Fleshman et al. 1966).

Phosphate and calcium are major components of bone mineral and exhibit broadly similar kinetic behavior in bone, despite their diametrically opposite chemical properties (Parfitt and Kleerekoper 1980). Rapid uptake of phosphate or calcium occurs on all bone surfaces. In both cases there is considerable variability in the initial concentration between different bones and between different surfaces, and both radiophosphorus and radiocalcium tend to accumulate in hot spots in forming bone. The fraction of the total mineral which is exchangeable may be about the same for phosphate as for calcium. Although phosphate ions can leave bone without calcium ions by heterionic exchange for citrate or carbonate, such loss appears to occur on a small scale. It seems reasonable to assume that the process of bone remodeling removes phosphate and calcium from bone at the same rate.

Based on these considerations, a generic bone model structure originally developed for application to calcium-like elements (Leggett 1992, 1993; ICRP 1993) is applied here to tungsten. Although the deposition fraction for bone is based on tungsten-specific information, the kinetics of tungsten after deposition in bone is assumed to be the same as that for calcium.

The model structure is an updated version of the alkaline earth model for adult man described in ICRP Publication 20 (1973). In this model, bone is divided into four primary parts: cortical surface, cortical volume, trabecular surface, and trabecular volume. The rapidly exchangeable material in bone is assumed to reside on bone surfaces, meaning endosteal and periosteal surfaces of cortical bone, surfaces of haversian and Volkmann canals, surfaces of resorption cavities, and surfaces of trabecular bone. Bone surfaces do not include the surfaces of lacunae or canaliculi and should not be confused with the surfaces of the sub-microscopic bone crystals. The more slowly exchangeable material in bone is assumed to reside in bone volume. Long-term loss from bone is associated primarily with bone resorption. In contrast to the ICRP's earlier model for alkaline earth elements, redeposition of material removed from bone compartments is treated explicitly, and material is assumed to be removed from all bone compartments by first-order processes.

In the generic model for calcium-like elements, cortical and trabecular bone volume are each viewed as consisting of two pools, referred to as the "exchangeable" and "nonexchangeable" pools (EXCH and NONEXCH in Fig. C.4). The exchangeable bone volume pool is used to account for most of the loss of elements from bone volume that occurs during the first few months after injection. Elements moving from bone surfaces to bone volume are assumed to enter the exchangeable pool and to leave this pool with an element-specific half-time (a few weeks or months). Part of the material leaving exchangeable bone volume is assumed to return to rapidly exchanging bone surfaces and part is assigned to the nonexchangeable bone volume, from which it is assumed to be removed to plasma only by bone resorption. The rate of removal from nonexchangeable bone volume is equated with the rate of bone turnover and is independent of the element considered.

Data on retention of tungsten in bone of laboratory animals are summarized in Fig. C.7 (Durbin 1960, Ballou 1960, Fleshman et al. 1966, Kaye 1968, Aamodt 1975, Ekman et al. 1977, Ando et al. 1989). The deposition data for rats and the retention data for larger animals, including semi-quantitative data on cows (Mullen et al. 1976) not shown in Fig. C.7, are reproduced reasonably well by using a deposition fraction of 0.08 for skeleton and assuming that the skeletal kinetics of tungsten is otherwise the same as that of calcium (Leggett 1992, ICRP 1993). 


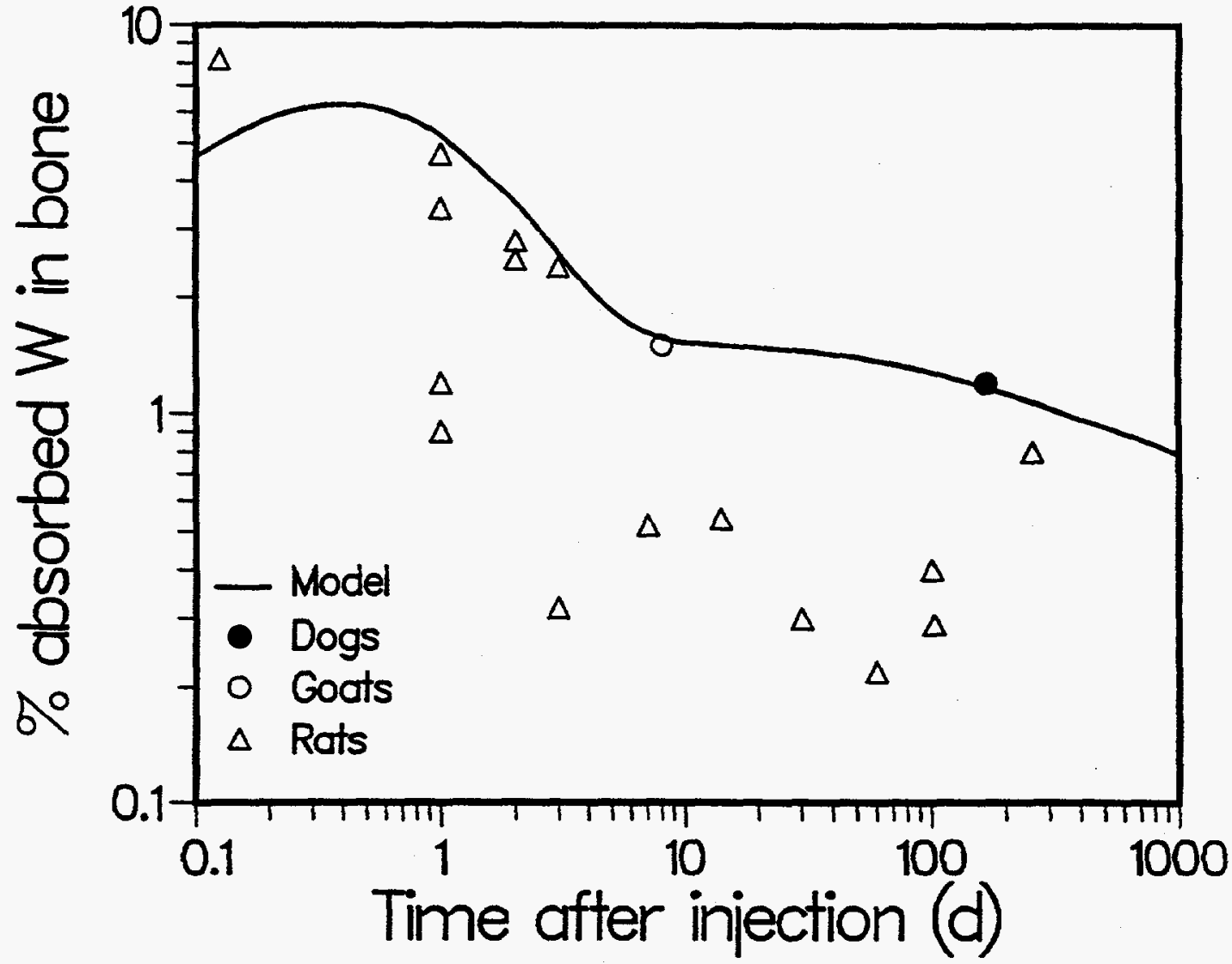

Fig. C.7. Data on retention of tungsten in bone of laboratory animals (Durbin 1960, Ballou 1960, Fleshman et al. 1966, Kaye 1968, Aamodt 1975, Ekman et al. 1977, Ando et al. 1989) and predictions of the present model for the case of intravenous injection of tungsten into a typical adult human.

Based on the model for calcium, the ratio of the deposition fractions for trabecular and cortical surfaces is assumed to be 1.25 for mature adults. This is consistent with limited data on the initial division of tungsten between trabecular and cortical bone (Ekman et al. 1977). The resulting transfer rate from plasma to trabecular bone surfaces is $1.25 / 2.25 \times 0.08 \times 11.648 \mathrm{~d}^{-1}=0.51769 \mathrm{~d}^{-1}$ and from plasma to cortical surfaces is $1.0 / 2.25 \times 0.08 \times 11.648 \mathrm{~d}^{-1}=0.41415 \mathrm{~d}^{-1}$.

Based on the calcium model, the removal half-time of tungsten from bone surfaces to all destinations (plasma and bone volume) is assumed to be $1 \mathrm{~d}$. Of tungsten leaving either cortical or trabecular bone surfaces, $5 / 6$ is assumed to return to plasma and $1 / 6$ is assumed to move to the corresponding exchangeable bone volume compartment. The transfer rate from cortical or trabecular surfaces to plasma is $(5 / 6) \times \ln (2) /(1 \mathrm{~d})=0.57762 \mathrm{~d}^{-1}$, and the transfer rate from cortical or trabecular bone surfaces to exchangeable bone volume is $(1 / 6) \times \ln (2) /(1 \mathrm{~d})=0.11552 \mathrm{~d}^{-1}$. The removal half-time assigned to each exchangeable bone volume compartment is $100 \mathrm{~d}$, with $60 \%$ of outflow assigned to the corresponding nonexchangeable bone volume compartment and $40 \%$ to the corresponding bone surface compartment. Therefore, the transfer rate from trabecular or cortical exchangeable bone volume to the corresponding bone surface compartment is $0.4 \times \ln (2) / 100 \mathrm{~d}=0.002773 \mathrm{~d}^{-1}$, and the transfer rate from the trabecular or cortical exchangeable bone volume to the corresponding nonexchangeable bone volume compartment is $0.6 \times \ln (2) / 100 \mathrm{~d}=0.004159 \mathrm{~d}^{-1}$. Removal from nonexchangeable bone to plasma is assumed to occur at the rate of bone turnover, which varies with age and is different for 
cortical and trabecular bone. For the average adult, the bone turnover rate is assumed to be $0.03 \mathrm{y}^{-1}$ for cortical bone and $0.18 \mathrm{y}^{-1}$ for trabecular bone (ICRP 1989, 1993). Thus, transfer rates from nonexchangeable bone volume to plasma are $8.21 \times 10^{-5} \mathrm{~d}^{-1}$ for cortical bone and $4.93 \times 10^{-4} \mathrm{~d}^{-1}$ for trabecular bone.

\section{C.5 RECOMMENDATIONS FOR FURTHER RESEARCH}

Three types of biokinetic models are used to predict the fate of inhaled or ingested tungsten in humans: a respiratory tract model that describes the deposition of inhaled tungsten and subsequent transfer to blood, the GI tract, or the environment; a GI tract model that describes the movement of ingested or endogenously secreted tungsten through the segments of the GI tract and transfer to blood or excreta; and a systemic model that describes the time-dependent distribution and excretion of absorbed tungsten. Due to the paucity of direct quantitative information on the biokinetics of tungsten in humans, parameter values for these models are based on two sources of indirect information: data on laboratory animals exposed to tungsten, and data on humans or laboratory animals exposed to physiological analogues of tungsten. The biokinetics of internally deposited tungsten has been studied in several different types of animals, including rats, mice, dogs, goats, pigs, sheep, cows, and mice. Because tungsten bears a close physiological resemblance to molybdenum and appears to mimic the behavior of phosphorus in bone to some extent, biokinetic data on these two extensively studied essential elements also provide some insight into the potential biokinetics of the nonessential element tungsten.

Additional experimental data are needed on several aspects of the biokinetics of tungsten, but there is a particular need for improved information on the biological fate of inhaled tungsten. Retention and absorption of inhaled tungsten have been investigated in detail only in one study, which involved inhalation of a single physico-chemical form of tungsten by dogs. It is not evident that the results of that study are representative of the behavior of forms of airborne tungsten that may be encountered by humans in environmental, occupational, or military settings. Experience with more frequently studied elements indicate that enormous differences in respiratory-tract kinetics, including fractional absorption of inhaled material to blood, can occur for different physical or chemical forms of the same element. There is a need to derive a wider range of experimental data on the biological fate of inhaled tungsten, using various physical and chemical forms of tungsten and additional large animal species. While inhalation experiments involving human subjects may not be feasible, it may be possible to gather better quantitative information from occupationally exposed persons than has been gathered to this point. For example, some information on the level of absorption of inhaled tungsten might be gained from bioassay measurements, particularly measurements of urinary excretion of tungsten, in workers acutely exposed to elevated levels of tungsten in air.

Data on GI uptake of tungsten in different types of animals suggest that, as an average, perhaps a onefourth to one-half of tungsten ingested in relatively soluble form is absorbed to blood. These data also indicate that fractional absorption can vary substantially from one situation to another, depending on such factors as the form of tungsten ingested, the nature of the diet, and the age of subject. Observations of GI uptake of tungsten in human consist of a single balance study in which measurements of urinary excretion of this element was compared with its level of intake in food. The results of this study suggest that roughly one-half of ingested tungsten is absorbed to blood. When conducted under closely controlled conditions over an extended period, such balance studies provide a useful, safe, and acceptable means of studying absorption of elements in humans. However, the 
previous balance study involved a large number of elements, and attention to tungsten was too limited and the study period was too short to provide much confidence that derived absorption values for tungsten are typical. It would be useful to conduct a more detailed balance study on human subjects that is designed specifically for investigation of GI uptake of tungsten.

Although based mainly on information on laboratory animals, the derived systemic biokinetic model for tungsten is expected to provide reasonable first estimates of the time-dependent distribution of absorbed tungsten in humans, particularly in the skeleton. This is because: (1) the data used to derive systemic transfer rates were mainly for animals that have usually proven to be reasonably "humanlike" with regard to biokinetics of absorbed metals (e.g., dogs and pigs); (2) data on the behavior of physiological analogues of tungsten in humans were available to serve as a broad indicator of tungsten biokinetics in humans; and (3) the model was developed within a biologically realistic framework that allowed data on tungstate retention in animal bone to be supplemented or replaced by quantitative human data on bone physiology.

Unfortunately, some of the larger uncertainties in predictions of the systemic biokinetic model are associated with soft tissues such as brain, testes, uterus, and fetus that are indicated by animal data as potential sites of toxic action of tungsten. Due to the paucity, inconsistency, or doubtful applicability to humans of biokinetic data for such organs, all soft tissues other than liver, kidneys, and spleen were assigned in the present biokinetic model to a single pool in which tungsten was assumed to be uniformly distributed. Clearly, more definitive experimental data on tungsten uptake and retention by laboratory animals are needed for soft tissues that appear to be most susceptible to toxic effects of this element. In addition, information on the steady-state distribution of tungsten in humans might be obtained from autopsy measurements of tungsten in human tissues. A limited amount of data on tungsten in human tissues or fluids is already available, but the data are too scattered to be of use in constructing a reasonable steady-state distribution. Moreover, the reliability of older data on traceelement concentrations in human tissues is questionable due to insensitivity of measurement techniques and, in many cases, lack of appreciation by researchers of the high potential for trace-element contamination of tissue samples.

Given the need for further experimental data on the biokinetics of tungsten in laboratory animals, the question arises as to the most useful animal model for man. The rat apparently is a poor model of tungsten biokinetics in larger animals and hence cannot be considered a reliable model of tungsten biokinetics in humans. On the other hand, the development of risk estimates for humans exposed to tungsten requires not only knowledge of the biokinetics of tungsten in humans but also knowledge of its biokinetics in the laboratory animals in which toxic effects of tungsten have been observed. This is because the tissue concentrations of tungsten often have not been measured in toxicity studies but must be estimated from information on the administered dosage. Because most of the available data on toxic effects of tungsten are for rats, it would be useful to have more detailed information on the timedependent distribution and retention of tungsten in rats. In addition, to improve the model of tungsten biokinetics in humans, it would also be useful to have more detailed information on the timedependent distribution and retention of tungsten in larger and more human-like animals.

In addition to improvement of biokinetic data for large animals, it would be useful to further investigate toxic effects of tungsten in animals other than rats. Clearly there are advantages in using the rat as a laboratory model for man, such as its relatively low cost, availability, and ease of handling. In addition, the toxicological database for tungsten as well as most other xenobiotics in rats provides a much better benchmark for interpretation of new results than is available for other types of laboratory 
animals. Thus, in a sense, the use of the rat as a laboratory model is self-perpetuating. On the other hand, there are many qualitative differences between rats and humans that limit our confidence in pharmacological and toxicological data on these animals. With regard to factors affecting the distribution, retention, and possibly the toxic effects of xenobiotics, rats generally have lower protein binding, higher biliary secretion, higher urinary concentrations, and lower urinary excretion of contaminants than do humans. Rats are obligatory nose breathers, which results in passage of the entire inhaled dose through the upper respiratory tract, while humans may breathe through the nose or mouth. Rats have numerous flora in the stomach and proximal small intestine, while humans have little or none. The skeletons of rats have somewhat different vasculature, modes of ossification, and patterns of development from those of humans and, unlike the human skeleton, the rat skeleton continues to grow throughout adulthood. Because of these and many other qualitative differences between rats and man, data for rats often are given less weight in development of biokinetic models for man than data on larger mammals such as monkeys, dogs, or pigs that show fewer and generally less striking anatomical and physiological differences from humans.

With regard to the biokinetics of tungsten, the initial distribution of absorbed tungsten may be similar in rats and larger animals, but rats appear to excrete tungsten at a much higher rate than do larger animals. In the development of a biokinetic model for humans, data for all species were considered in selection of parameter values that determine the initial distribution of tungsten, but only data for larger animals were used to derive parameter values controlling the rate of loss of tungsten from the body.

Species differences in the biokinetics of a substance do not necessarily imply corresponding species differences in toxicity per unit tissue concentration but do raise a cautionary flag in that regard.

Differences in biokinetics of a substance could reflect qualitative differences in the behavior of that substance, such as differences in membrane transport or protein binding. Such qualitative differences could well result in differences in toxicity per unit tissue concentration. On the other hand, observed species differences in biokinetics of a substance may often reflect only quantitative differences in the rates of qualitatively similar phenomena, which seems less likely to be associated with differences in susceptibility to toxic effects of a substance.

Given the pros and cons of the rat as a toxicological model for tungsten in man, it may be prudent to use a two-pronged approached to further experiments on toxicity of tungsten, i.e., build on the available database for rats with further experiments on rats but at the same time perform more limited parallel experiments on larger animals. If the comparative data suggest that the rat is an inappropriate toxicological model for larger animals, then a better model should be found.

\section{C.6 REFERENCES}

Aamodt, R. L. Retention and Excretion of injected ${ }^{181} \mathrm{~W}$ labeled sodium tungstate by beagles. Health Physics 24:519-524; 1973.

Aamodt, R. L. Inhalation of ${ }^{181} \mathrm{~W}$ labeled tungstic oxide by six beagle dogs. Health Physics $28: 733-$ $742 ; 1975$.

Ando, A.; Ando, I; Hiraki, T; Hisada, K. Relation between the location of elements in the periodic table and various organ-uptake rates. Nucl. Med. Biol. 16:57-80; 1989. 
Ballou, J. E. Metabolism of $\mathrm{W}^{185}$ in the rat. AEC Research and Development Report HW-64112; 1960

Bell, M. C.; Sneed, N. N. Metabolism of tungsten by sheep and swine. Trace Elements Metabolism in Animals 70-72; 1970.

Bell, M. C.; Sneed, N. N.; Hall, R. F. Effect of ration and route of administration on metabolism of ${ }^{99}$ Mo by sheep and swine. Proc. 7th Snt. Cong. Nutr. 5:765-770; 1966.

Bell, M. C.; Diggs, B. G.; Lowrey, R. S.; Wright, P. L. Comparison of $\mathrm{Mo}^{99}$ metabolism in swine and cattle as affected by stable molybdenum. J. Nutrition $84: 367-372 ; 1964$.

Bibr, B.; Deyl, Z.; Lener, J.; Kucera, J.; Simkova, M. The mechanism of action of molybdenum and tungsten upon collagen structures in vivo. Physiologia Bohemoslovaca 36:417-424; 1987.

Cardin, C. J.; Mason, J. Molybdate and tungstate transfer by rat ileum competitive inhibition by sulphate. Biochimica et Biophysica Acta 455:937-946; 1976.

Chertok, R. J.; Lake, S. Availability in the peccary pig of radionuclides in nuclear debris from the plowshare excavation buggy. Health Physics 20:313-316; 1971a.

Chertok, R. J.; Lake, S. Biological availability of radionuclides produced by the plowshare event schooner - I. Body distribution in domestic pigs exposed in the field. Health Physics 20:317$324 ; 1971 b$.

Chertok, R. J.; Lake, S. Biological availability of radionuclides produced by the plowshare event schooner - II Retention and excretion rates in peccaries after a single oral dose of debris. Health Physics 20:325-330; 1971c.

Cugell, D. W.; Morgan, W. K. C.; Perkins, D. G.; Rubin, A. The respiratory effects of cobalt. Arch. Intern. Med. 150:177-183; 1980.

De Sousa Pereira, A.; Grande, N. R.; Carvalho, E.; Ribeiro, A. Evidence of drainage of tungsten particles introduced in the pleural space through the visceral pleura into the lung parenchyma. Acta. Anat. 145:416-419; 1992.

Durbin, P. W. Metabolic characteristics within a chemical family. Health Physics 2:225-238; 1960.

Durbin, P.W.; Scott, K. G.; Hamilton, J. G. The distribution of radioisotopes of some heavy metals in the rat. Univ. Calif. Pub. Pharmacol 3; 1957.

Ekman, L.; Figueiras, H. D.; Jones, B. E. V.; Myamoto, S. Metabolism of ${ }^{181}$ W-labeled sodium tungstate in goats. FOA Report C-40070-A3; November 1977.

Edel, J.; Sabbioni, E.; Pietra, R.; Rossi, A.; Torre, M.; Rizzato, G.; Fraioli, P. Trace metal lung disease: in vitro interaction of hard metals with human lung and plasma components. The Science of the Total Environment 95:107-117; 1990. 
Eve, I. S. A review of the physiology of the gastrointestinal tract in relation to radiation doses from radioactive materials. Health Phys. 12:131-161; 1966.

Fleshman, D; Krotz, S.; Silva, A. The metabolism of elements of high atomic number. UCRL 14739:69-86; 1966.

Grande, N. R.; Moreira de Sá, C.; Ánguas, A. P.; Carvalho, E.; Soares, M. Time course and distribution of tungsten-laden macrophages in the hilar lymph nodes of the dog lung after experimental instillation of calcium tungstate into the left apical bronchus. Lymphology 23:171$182 ; 1990$.

International Commission on Radiological Protection. Alkaline earth metabolism in adult man. ICRP Publication 20. Oxford: Pergamon Press; 1973.

International Commission on Radiological Protection. Human respiratory tract model for radiological protection. ICRP Publication 66. Oxford: Pergamon Press; 1994.

International Commission on Radiological Protection. Limits for intakes of radionuclides by workers. Publication 30, Part 1. Oxford: Pergamon Press; 1979.

International Commission on Radiological Protection. Limits for intakes of radionuclides by workers. Publication 30, Part 3. Oxford: Pergamon Press; 1981.

International Commission on Radiological Protection. Age-dependent doses to members of the public from intake of radionuclides. Part 2: ICRP Publication 67. Oxford: Pergamon Press; 1993.

International Commission on Radiological Protection. Age-dependent doses to members of the public from intake of radionuclides. Part 3: ICRP Publication 69. Oxford: Pergamon Press; 1995.

Johnson, J. L.; Cohen, H. J.; Rajagopalan, K. V. Molecular basis of the biological function of molybdenum. Molybdenum-free sulfite oxidase from livers of tungsten-treated rats. J. Biol. Chem. 249:5046-5055; 1974a.

Johnson, J. L.; Waud, W. R.; Cohen, H. J.; Rajagopalan, K. V. Molecular basis of the biological function of molybdenum. Molybdenum-free xanthine oxidase from livers of tungsten-treated rats. J. Biol. Chem. 249:5056-5061; 1974b.

Johnson, J. L.; Rajagopalan, K. V. Molecular basis of the biological function of molybdenum. Effect of tungsten on xanthine oxidase and sulfite oxidase in the rat. J. Biol. Chem. 249:859-866; 1974.

Kaye, S. V. Distribution and retention of orally administered radiotungsten in the rat. Health Physics 15:399-417; 1968 .

Kollmer, W. E.; Schramel, P.; Samsahl, K. Simultaneous determination of nine elements in some tissues of the rat using neutron activation analysis. Phys. Med. Biol. 17:555-562, 1972. 
Leggett, R. W. A generic age-specific biokinetic model for calcium-like elements, Radiation Protection Dosimetry 41:183-198; 1992.

Leggett, R. W. An age-specific kinetic model of lead metabolism in humans. Environ. Health Perspect. 101:598-616; 1993.

Leggett, R. W. Basis for the ICRP's age-specific biokinetic model for uranium. Health Physics 67:589$610 ; 1994$.

Mason, J.; Mulryan, G.; Lamand, M.; LaFarge, C. Behavior of [185W] thiotungstates injected into sheep and the influence of copper: their fate and the effect of the compounds upon plasma copper. Journal of Inorganic Biochemistry 35:115-126; 1989.

Mullen, A. L.; Bretthauer, E. W.; Stanley, R. E. Absorption, distribution and milk secretion of radionuclides by the dairy cow - V. Radiotungsten. Health Physics 31:417-424; 1976.

Nicolaou, G.; Pietra, R.; Sabbioni, E.; Mosconi, G.; Cassina, G.; Seghizzi, P. Multielement determination of metals in biological specimens of hard metal workers: A study carried out by neutron activation analysis. J. Trace Elem. Electrolytes Health Dis. 1:73-77; 1987.

Parfitt, A. M.; Kleerekoper, M. The divalent ion homeostatic system-physiology and metabolism of calcium, phosphorus, magnesium, and bone. In: Clinical disorders of fluid and electrolyte metabolism, ed. by M. H. Maxwell and C. R. Kleeman. New York: McGraw-Hill; 1980; 269398.

Peão, M. N. D.; Águas, A. P.; de Sá, C. M.; Grande, N. R. Inflammatory response of the lung to tungsten particles: an experimental study in mice submitted to intratracheal instillation of a calcium tungstate powder. Lung 171:187-201; 1993.

Rosoff, B.; Spencer, H. The distribution and excretion of molybdenum-99 in mice. Health Physics 25:173-175; 1973.

Ryan, J.; McKillen, M.; Mason, J. Sulphate/molybdate interactions: in vivo and in vitro studies on the group VI oxyanion transport system in ovine renal tubule epithelial cells. Ann. Rech. Vet. 18:47-55; 1987.

Scott, K. G. The metabolic properties of various metals. USAEC, UCRL 1694: 7-11; 1952.

Sjögren, I.; Hillerdal, G.; Andersson, A.; Zetterström. Hard metal lung disease: importance of cobalt in coolants. Thorax 35:653-659; 1980.

Tumlund, J. R.; Keyes, W. R.; Peiffer, G. L.; Chiang, G. Molybdenum absorption, excretion, and retention studied with stable isotopes in young men during depletion and repletion ${ }^{1-4}$. Am. J. Clin. Nutr. 61:1102-1109; 1995.

Wase, A. W. Absorption and distribution of radio-tungstate in bone and soft tissues. Archives of Biochemistry and Biophysics 61:272-277; 1956. 
Wester, P.O. Trace element balances in relation to variations in calcium intake. Atherosclerosis 20:207-215; 1974. 


\begin{abstract}
APPENDIX D
COMPARISON OF LIMITS ON INTAKE OF URANIUM BASED ON RADIOLOGICAL AND CHEMICAL GUIDANCE
\end{abstract}

R. W. Leggett and K. F. Eckerman 



\section{APPENDIX D. COMPARISON OF LIMITS ON INTAKE OF URANIUM BASED ON RADIOLOGICAL AND CHEMICAL GUIDANCE}

\section{D.1 INTRODUCTION}

There are two hazards associated with intake of uranium: chemical interference with normal renal function; and radiogenic injury to lungs, bone, and other tissues. The relative significance of the chemical and radiological hazards depends on the mixture of uranium isotopes and the chemical form of uranium taken into the body. Chemical toxicity generally has been considered the overriding hazard for fairly soluble uranium compounds with nearly naturally occurring isotopic mixtures, based on studies with experimental animals (Wrenn et al. 1985). The radiogenic risk increases with the level of ${ }^{235} \mathrm{U}$ enrichment due mainly to an associated increase in the percentage of ${ }^{234} \mathrm{U}$, which has a much higher specific activity than ${ }^{235} \mathrm{U}$ and ${ }^{238} \mathrm{U}$. Also, for the case of inhalation of insoluble uranium compounds, the radiation dose to the lungs could become the prevailing consideration even for natural or depleted uranium due to an increased residence time in the lungs and a decreased level of absorption to blood compared with soluble compounds.

The purpose of this appendix is to compare radiological and chemical limits on intake of uranium for different modes of intake, solubility levels, and isotopic mixtures of uranium (including depleted uranium). The limits on intake considered here are for occupational exposures, but conclusions concerning the relative importance of radiological and chemical limits should also apply to non-occupational exposures. This is because limits on non-occupational exposure to uranium generally are derived by applying an additional safety factor to derived limits on occupational exposure, and it seems reasonable to apply the same safety factor whether considering radiological or chemical hazards of uranium.

The derived limits on intake of uranium are based on the following:

1. the ICRP's current guidance on occupational exposures to radiation (ICRP 1991);

2. longstanding guidance on occupational exposure to uranium as a chemical hazard to the kidneys (ICRP 1959), with modifications for consideration of chronic exposures based on current information;

3. the ICRP's new respiratory tract model (ICRP 1994);

4. the ICRP's gastrointestinal tract model (ICRP 1979), with an updated GI uptake factor for soluble uranium (ICRP 1995);

5. a relatively detailed version of the ICRP's new systemic biokinetic model for uranium (Leggett 1994);

6. estimates of typical mass ratios of ${ }^{234} U$ and ${ }^{235} U$ in depleted, natural, and enriched uranium.

\section{D.2 PRIMARY GUIDANCE FOR PROTECTION AGAINST CHEMICAL AND RADIOLOGICAL TOXICITY OF URANIUM}

\section{D.2.1 Primary Guidance for Uranium as a Radiological Hazard}

To place all ionizing radiations on a common scale with regard to their potential health detriment, the ICRP uses quantities called the equivalent dose and the effective dose. The equivalent dose is the 
absorbed dose averaged over an organ or tissue and multiplied by a radiation weighting factor that reflects the relative biological effectiveness of the type and energy of radiation causing the dose. The effective dose takes into account that the relationship between equivalent dose and the probability of radiogenic effects depends on the organ or tissue irradiated. The effective dose is a weighted sum of equivalent doses to radiosensitive tissues, with the tissue weighting factor representing the relative contribution of that tissue to the total detriment for the case of uniform irradiation of the whole body.

The ICRP's current primary guidance on occupational exposures to radiation (ICRP 1991) provides for some exceptional cases but essentially limits the effective dose (50-y integral) to $0.02 \mathrm{~Sv}$ from intakes over a one-year period. This radiological guidance level will be applied throughout this analysis.

\section{D.2.2 Primary Guidance for Uranium as a Chemical Hazard}

Exposure guidelines for uranium as a chemical hazard generally have been based on a permissible concentration in kidney, which appears to be the organ most susceptible to chemical effects of this element (Voegtlin and Hodge 1953, Spoor and Hursh 1973, Stopps and Todd 1982). Toxic effects on the kidneys are assumed to occur only when the renal uranium concentration exceeds some threshold level.

Since the early 1950 s a concentration of $3 \mu \mathrm{g} \mathrm{U}$ per g kidney has served as a primary guidance level for avoidance of chemical toxicity in workers exposed to uranium (Voegtlin and Hodge 1953, Spoor and Hursh 1973, Stopps and Todd 1982). According to Spoor and Hursh (1973), this level represents a committee's judgment based primarily on results of animal experiments conducted in the 1940's. There have been several cases in which adult humans experienced renal concentrations estimated to exceed $3 \mu \mathrm{g} \mathrm{g}^{-1}$ for periods of up to a few days, without showing long-term adverse health effects. Thus, there is some evidence that the longstanding guidance level of $3 \mu \mathrm{g} \mathrm{U} \mathrm{g}^{-1}$ kidney may be suitable for consideration of acute exposures. On the other hand, there are no case studies on human subjects who have chronically experienced renal uranium concentrations exceeding $3 \mu \mathrm{g} \mathrm{g}^{-1}$, or even one-tenth of that level, and there is no basis for assuming that the threshold level for renal toxicity is the same for chronic exposures as for acute exposures (Leggett 1989, Foulkes 1990). Moreover, results of animal studies suggest that mild renal injury may occasionally occur in chronically exposed animals at renal uranium concentrations of a few tenths of a microgram per gram kidney (Leggett 1989, Foulkes 1990).

Recently, several reviewers and analysts have proposed that the longstanding chemical guidance level for uranium be reduced, particularly for consideration of chronic exposures (Morrow et al. 1982, Wrenn et al. 1985, Morrow 1984, Sula et al. 1989, Leggett 1989, SuLu and Zhao 1990, Foulkes 1990). The recently proposed guidance values are in the range $0.25-1.0 \mu \mathrm{g} \mathrm{U} \mathrm{g}^{-1}$ kidney.

In the present analysis, we apply a value of $0.3 \mu \mathrm{g} \mathrm{U} \mathrm{g} \mathrm{g}^{-1}$ kidney as the primary guidance level for prevention of chemical toxicity from chronic intake of uranium by a worker. For consideration of acute intake, we apply the older guidance level of $3 \mu \mathrm{g} \mathrm{U} \mathrm{g}^{-1}$ kidney. In effect, the renal uranium concentration would be allowed to exceed $0.3 \mu \mathrm{g} \mathrm{g}^{-1}$ by up to an order of magnitude for short periods, but intake would be limited so that the estimated steady-state concentration would not exceed $0.3 \mu \mathrm{g} \mathrm{g}^{-1}$. 


\section{D.2.3 A Common Unit for Comparison of Chemical and Radiological Limits}

For the purpose of reducing radiological and chemical guidance to common terms, guidelines for intake of uranium are expressed here in terms of an Annual Limit on Intake or ALI (with the exception of acute intakes, as described later). For brevity, intake limits based on primary guidance for uranium as a radiological hazard are called radiological ALIs, and those based on primary guidance for uranium as a chemical toxin are called chemical ALIs. The ALI for a given uranium isotope or isotopic mixture is defined as the minimum of the radiological and chemical ALIs. For purposes of comparison with chemical ALIs, radiological ALIs are converted from activity (Bq) to mass (g).

The radiological ALI for a uranium isotope or mixture of uranium isotopes is defined as the amount of activity which, if taken in during a one-year period, would yield the limiting effective dose of $0.02 \mathrm{~Sv}$. For all practical purposes, the radiological ALI is independent of the pattern of intake during the year. For example, the ALI could be taken in during a one-day period or at a constant rate over $365 \mathrm{~d}$.

Thus, there is no need to distinguish between a radiological limit on chronic intake and a radiological limit on acute intake.

The situation is more complicated with regard to chemical guidance, in that a given mass of uranium that does not violate the limit of $0.3 \mu \mathrm{g} \mathrm{U} \mathrm{g}^{-1}$ kidney if inhaled or ingested over a one-year period might yield a transient renal uranium concentration exceeding $3 \mu \mathrm{g} \mathrm{g}^{-1}$ if inhaled or ingested acutely. Thus, a "Limit on Acute Intake (LAI)" as well as an ALI should be set to avoid chemical effects of uranium on the kidneys.

According to the biokinetic model for uranium applied in this paper (Leggett 1994, ICRP 1995), the renal uranium concentration in a constantly exposed adult subject will increase continually with time until a "steady-state" or "asymptotic" concentration is virtually attained. The chemical ALI for a uranium isotope or mixture of uranium isotopes is the annual intake of uranium corresponding to a steady-state concentration of $0.3 \mu \mathrm{g} \mathrm{g}^{-1}$, assuming a constant intake rate. The chemical limit on acute inhalation or ingestion is the mass of acutely inhaled or ingested uranium that corresponds to an estimated peak renal uranium concentration of $3 \mu \mathrm{g} \mathrm{g}^{-1}$. Thus, for comparison of chemical and radiological limits on acute intake, we will use the term "Limit on Acute Intake" because the term "Annual Limit on Intake" would apply only to the radiological limit.

\section{D.3 UPDATED BIOKINETIC MODELS}

\section{D.3.1 Respiratory Tract Model}

The ICRP's new respiratory tract model (ICRP 1994), described in Appendix C, is applied here. All three default absorption types (F, M, and $S$, indicating fast, moderate, and slow absorption to blood, respectively) are considered. Inhaled particles are assumed in this analysis to have an activity median aerodynamic diameter (AMAD) of $5 \mu \mathrm{m}$.

\section{D.3.2 Gastrointestinal Tract Model}

The ICRP's gastrointestinal tract, described in Appendix $C$, is applied here. An $f_{1}$ value of 0.02 is applied to soluble forms of uranium (ICRP 1995) and a value of 0.002 is applied to relatively insoluble forms (ICRP 1979). For consideration of inhaled material moving from the lungs to the GI 
tract, an $f_{1}$ value of 0.02 is applied to absorption Types $F$ and $M$ and a value of 0.002 is applied to Type S.

\section{D.3.3 Biokinetic Model for Systemic Uranium}

The ICRP has recently adopted a new, physiologically descriptive, age-specific biokinetic model for uranium (Leggett 1994, ICRP 1995). Two versions of the model are available (Leggett 1994), differing mainly in the level of detail regarding kinetics of uranium in the blood and the kidneys. The two versions yield virtually identical estimates of effective dose and, for chronic intake scenarios, similar predictions of organ contents for times $\geq 1 \mathrm{~d}$ after the beginning of exposure. The more detailed version of the model is applied here because it is expected to yield better estimates of the peak renal uranium concentration after acute intake of uranium.

The structure of the relatively detailed model is shown in Fig. D.1, and transfer rates for the reference adult are given in Table D.1.

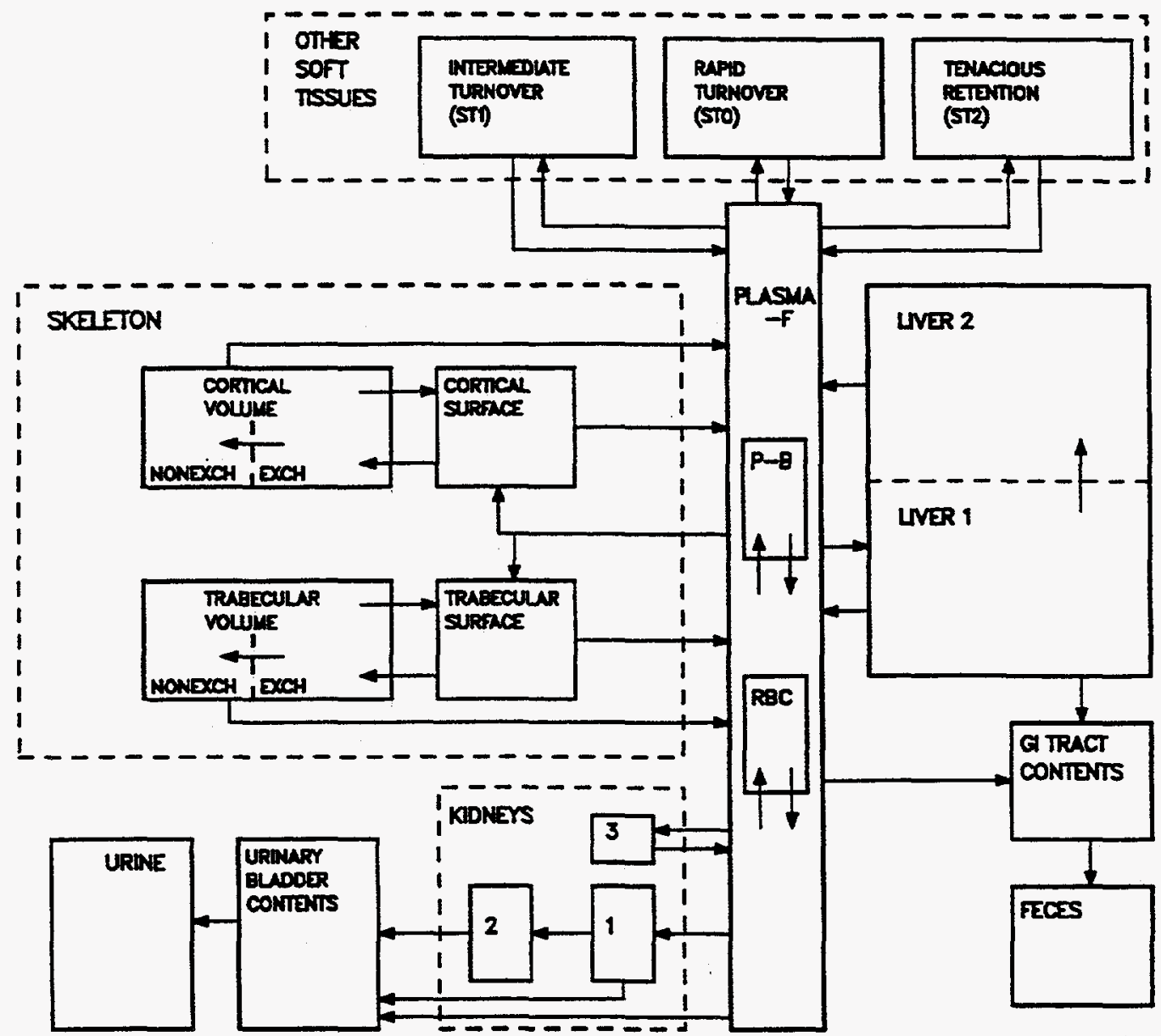

Fig. D.1. Diagram of the biokinetic model for systemic uranium (Leggett 1994). 
Table D.1. Transfer rates $\left(\mathrm{d}^{-1}\right)$ for the systemic biokinetic model for tungsten

\begin{tabular}{ll}
\hline Plasma to STO & $4.9920 \mathrm{E}+00$ \\
Plasma to RBC & $5.8240 \mathrm{E}-02$ \\
Plasma to urinary bladder & $8.7364 \mathrm{E}+00$ \\
Plasma to kidneys 1 & $5.2416 \mathrm{E}-01$ \\
Plasma to kidneys 2 & $5.8240 \mathrm{E}-02$ \\
Plasma to ULI cont & $5.8240 \mathrm{E}-01$ \\
Plasma to spleen & $5.8240 \mathrm{E}-03$ \\
Plasma to liver 1 & $4.6592 \mathrm{E}-01$ \\
Plasma to ST1 & $2.6208 \mathrm{E}-01$ \\
Plasma to ST2 & $2.3296 \mathrm{E}-02$ \\
Plasma to T bone surf & $5.1769 \mathrm{E}-01$ \\
Plasma to C bone surf & $4.1415 \mathrm{E}-01$ \\
STO to plasma & $8.3200 \mathrm{E}+00$ \\
RBC to plasma & $3.4657 \mathrm{E}-01$ \\
Kidneys 1 to UB cont & $1.3863 \mathrm{E}+00$ \\
Kidneys 2 to plasma & $1.9000 \mathrm{E}-03$ \\
Liver 1 to plasma & $3.1192 \mathrm{E}-01$ \\
Liver 1 to liver 2 & $3.4657 \mathrm{E}-02$ \\
ST1 to plasma & $6.9315 \mathrm{E}-02$ \\
ST2 to excreta & $1.9000 \mathrm{E}-03$ \\
Spleen to plasma & $1.9000 \mathrm{E}-03$ \\
T bone surf to plasma & $5.7800 \mathrm{E}-01$ \\
T bone surf to exch T bone vol & $1.1600 \mathrm{E}-01$ \\
C bone surf to plasma & $5.7800 \mathrm{E}-01$ \\
C bone surf to exch C bone vol & $1.1600 \mathrm{E}-01$ \\
Liver 2 to plasma & $1.9000 \mathrm{E}-03$ \\
Nonexch T bone vol to plasma & $4.9300 \mathrm{E}-04$ \\
Nonexch C bone vol to plasma & $8.2100 \mathrm{E}-05$ \\
Exch T bone vol to $T$ bone surf & $2.7730 \mathrm{E}-03$ \\
Exch T bone vol to nonexch T bone vol & $4.1590 \mathrm{E}-03$ \\
Exch C bone vol to C bone surf & $2.7730 \mathrm{E}-03$ \\
Exch C bone vol to nonexch C bone vol & $4.1590 \mathrm{E}-03$ \\
\hline
\end{tabular}




\section{D.4 TYPICAL MASS RATIOS OF ${ }^{234} \mathrm{U}$ AND ${ }^{235} \mathrm{U}$ IN DEPLETED, NATURAL, AND ENRICHED URANIUM}

In this analysis, natural uranium is assumed to contain $0.0057 \%{ }^{234} \mathrm{U}$ and $0.72 \%{ }^{235} \mathrm{U}$ by mass. Isotopic ratios used here for depleted and enriched uranium are intended to be representative of end products of the gaseous diffusion technique and may not be applicable to other separation techniques. For example, the gaseous diffusion technique leaves substantial quantities of ${ }^{234} U$ in ${ }^{235} U$-enriched uranium, while the laser isotopic separation technique proposed for future enrichment facilities is expected to separate only ${ }^{235} \mathrm{U}$, leaving ${ }^{234} \mathrm{U}$ with the depleted uranium (Rich et al. 1988).

Depleted uranium is assumed to contain $0.25 \%{ }^{235} \mathrm{U}$ and $0.0005 \%{ }^{234} \mathrm{U}$ by mass (Rich et al. 1988). The ratio of ${ }^{234} \mathrm{U}$ to ${ }^{235} \mathrm{U}$ in uranium enriched by the gaseous diffusion method can vary by $50 \%$ or more for a given level of ${ }^{235} U$. In this paper, the ${ }^{234} U$ content is based on the equation $\%{ }^{234} U=0.0015+$ $0.0058 \mathrm{E}+0.000054 \mathrm{E}^{2}$, where $\mathrm{E}=\%^{235} U$ by mass. This equation agrees within a few percent with a commonly applied model of the total specific activity in enriched uranium (Rich et al. 1988) and reproduces the isotopic ratios of natural uranium given above.

\section{D.5 RESULTS: COMPARISON OF RADIOLOGICAL AND CHEMICAL LIMITS ON INTAKE OF URANIUM}

Results of the analysis are summarized in Figs. D.2. through D.5. Comparisons of radiological and chemical limits on intake for the cases of chronic and acute inhalation of uranium are made in Figs. D.2 and D.3, respectively. Comparisons for the cases of chronic and acute ingestion are made in Figs. D.4 and D.5, respectively.

In these figures, the labels "Rad" and "Chem" refer to limits on intake based on radiological and chemical guidance, respectively. In Figs. D.2 and D.3, the labels "Rad: $X$ " and "Chem: $X$ " $(X=F, M$, or S) refer to radiological and chemical limits, respectively, for inhalation of uranium of absorption type $X$. For a given exposure mode (inhalation or ingestion), form of uranium (Type $F, M$, or $S$ for inhalation and soluble or insoluble for ingestion), and isotopic mixture (based on per cent ${ }^{235} \mathrm{U}$ by weight), the limit on intake is determined by the lower of the points on the curves labeled "Rad" and "Chem." For example, the limit on chronic inhalation of depleted uranium of Type F is determined by chemical guidance, because the solid curve labeled "Chem: F" in Fig. D.2 is below the solid curve labeled "Rad: F" at the point 0.25 on the $x$-axis.

For the case of chronic inhalation (Fig. D.2), chemical guidance is limiting for depleted or natural uranium of Type $F$ and $M$, while radiological guidance prevails for Type $F$ and $M$ material at enrichment levels exceeding about $42 \%$ and $3.5 \%$, respectively. For Type $S$ material, radiological guidance is limiting at all isotopic ratios due to the high radiation dose delivered to the lungs by tenaciously retained material.

In the case of acute inhalation (Fig. D.3), chemical guidance is limiting for all isotopic mixtures of the rapidly absorbed Type $\mathrm{F}$ material. For the more slowly absorbed Type $\mathrm{M}$ material, chemical guidance is limiting at enrichment levels below about $7 \%$ but is less restrictive than radiological guidance at higher enrichment levels. As in the case of chronic inhalation, radiological guidance is limiting at all isotopic ratios of the slowly absorbed Type $S$ material. 


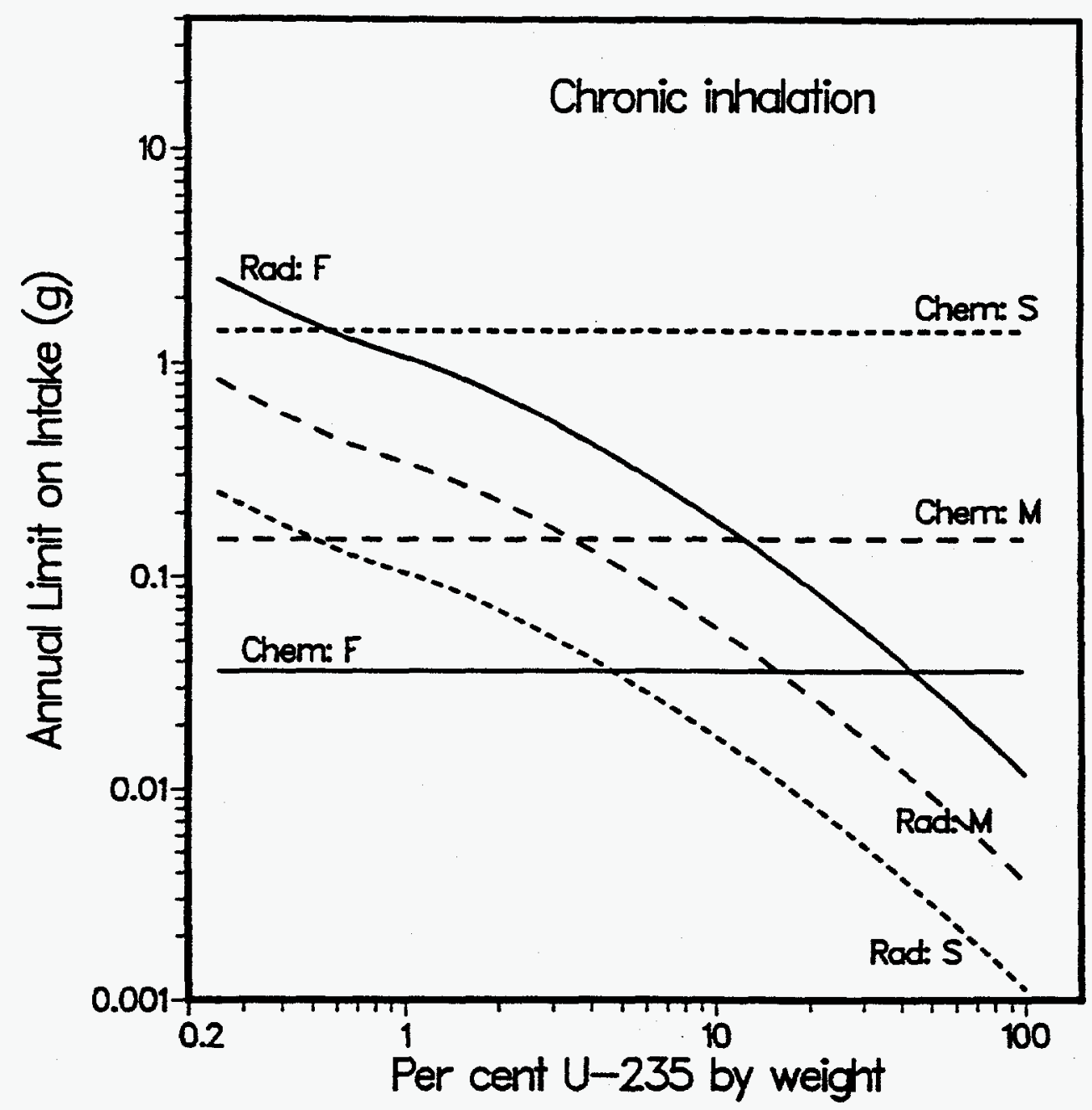

Fig. D.2. Comparison of limits on intake of uranium by chronic inhalation based on radiological (Rad) and chemical (Chem) guidance for different isotopic mixtures and absorption types ( $F$ is fast, $M$ is moderate, and $S$ is slow absorption). Calculations are based on a particle size of $5 \mu \mathrm{m}$ (AMAD).

For either chronic or acute ingestion (Figs. D.4 and D.5, respectively), chemical guidance is limiting for both soluble or insoluble forms of uranium except at relatively high levels of ${ }^{235} \mathrm{U}$ enrichment.

Recall that the analysis for inhaled materials was based on a particle size (AMAD) of $5 \mu \mathrm{m}$. Chemical as well as radiological limits vary to some extent with particle size, as do the specific levels of enrichment at which radiological guidance becomes the limiting factor. However, the general conclusions drawn above concerning the relative importance of chemical and radiological limits are actually fairly independent of particle size, at least within the range of particle sizes commonly observed in occupational or environmental settings. For example, for either $1-\mu \mathrm{m}$ or $5-\mu \mathrm{m}$ particles (AMAD), chemical guidance is limiting for all isotopic mixtures of Type F material, and radiological guidance is limiting for all isotopic ratios of Type $\mathrm{S}$ material. For Type $\mathrm{M}$ material, chemical guidance is limiting at enrichment levels below about $3 \%$ for $1-\mu \mathrm{m}$ particles and at enrichment levels below about $7 \%$ for $5-\mu \mathrm{m}$ particles, while radiological guidance is limiting at higher enrichment levels. 


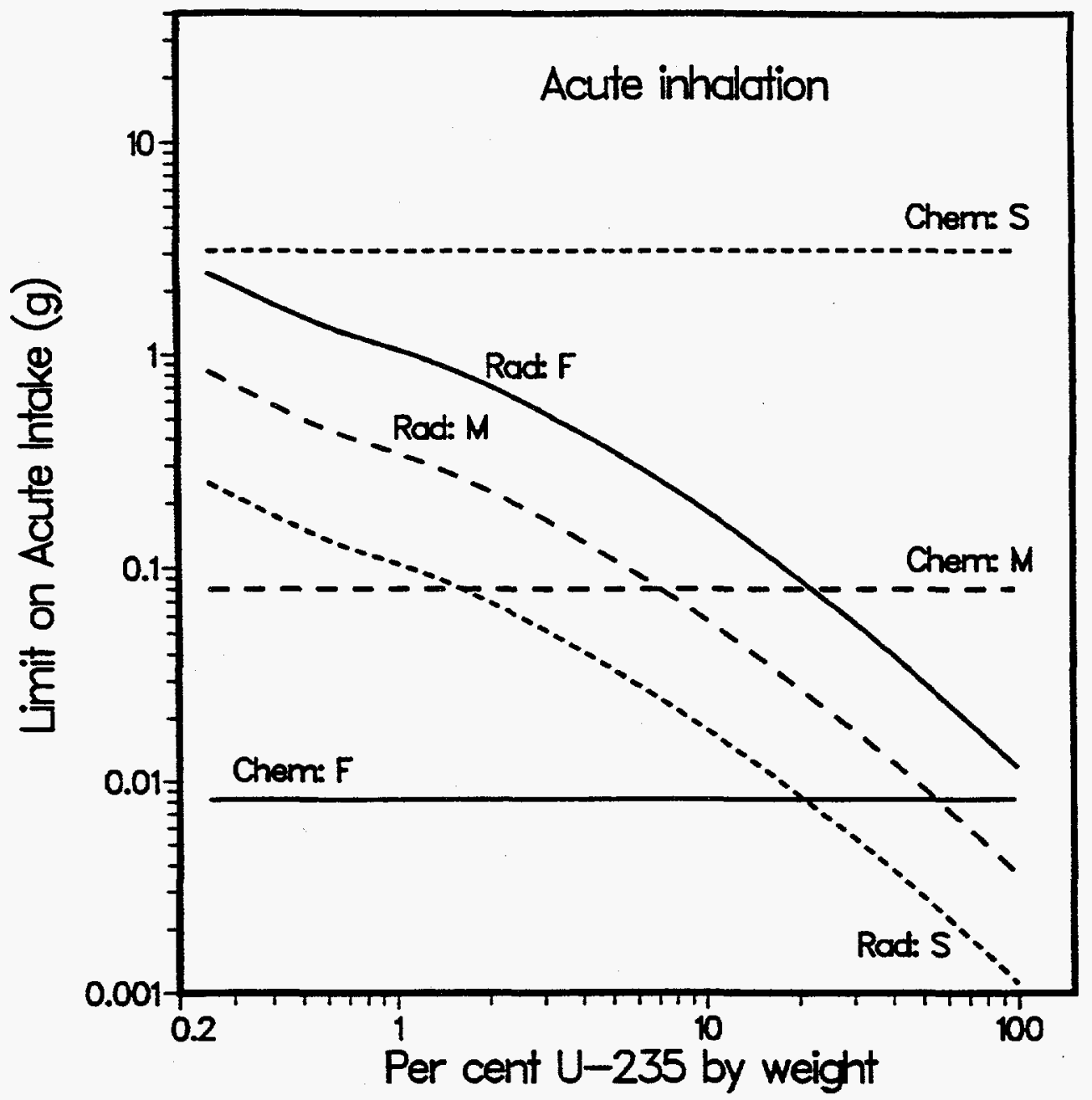

Fig. D.3. Comparison of limits on acute inhalation of uranium based on radiological (Rad) and chemical (Chem) guidance for different isotopic mixtures and absorption types ( $F$ is fast, $M$ is moderate, and $S$ is slow absorption). Calculations are based on a particle size of $5 \mu \mathrm{m}$ (AMAD). 


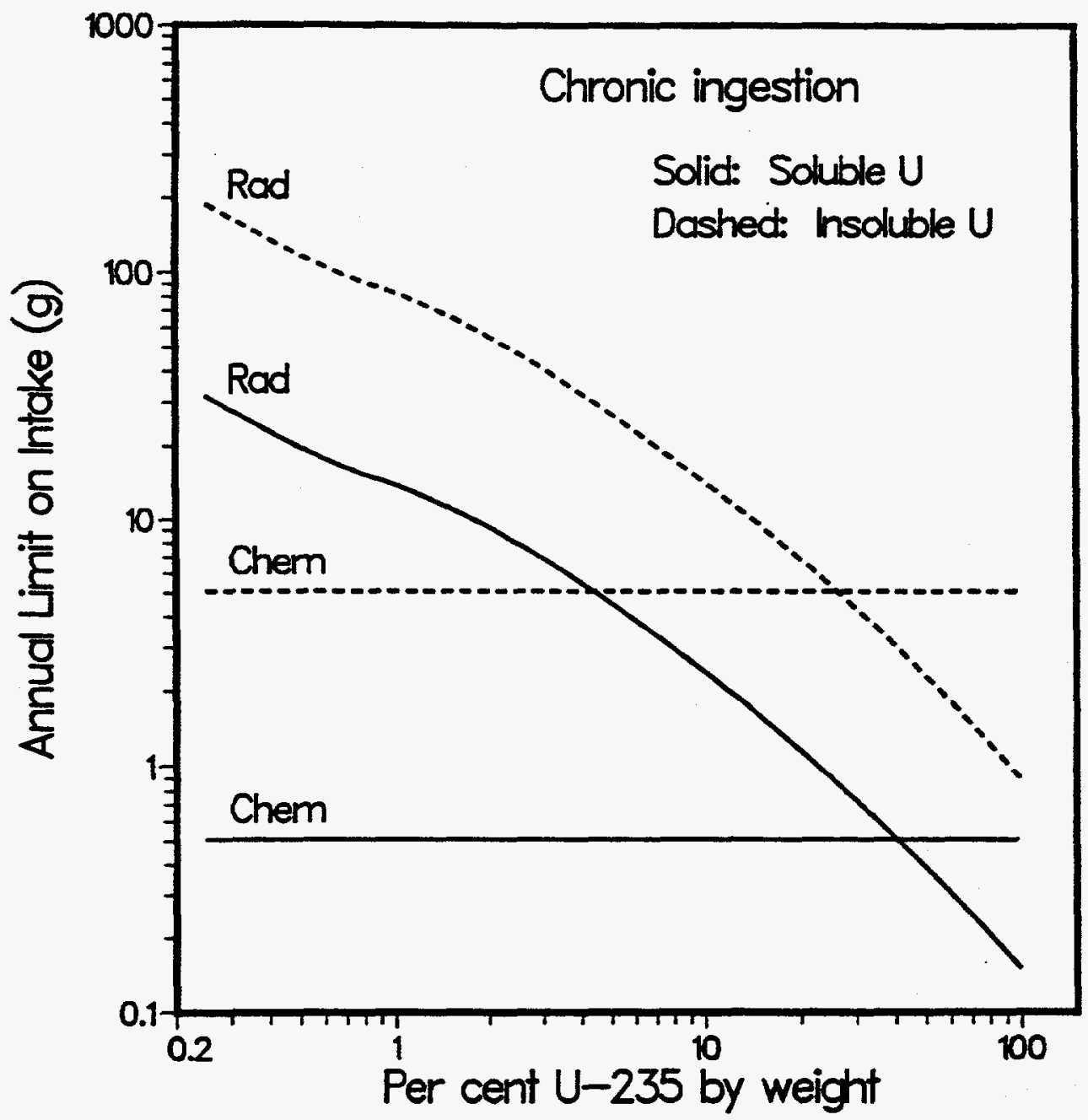

Fig. D.4. Comparison of limits on intake of uranium by chronic ingestion based on radiological (Rad) and chemical (Chem) guidance for different isotopic mixtures and solubility levels. 


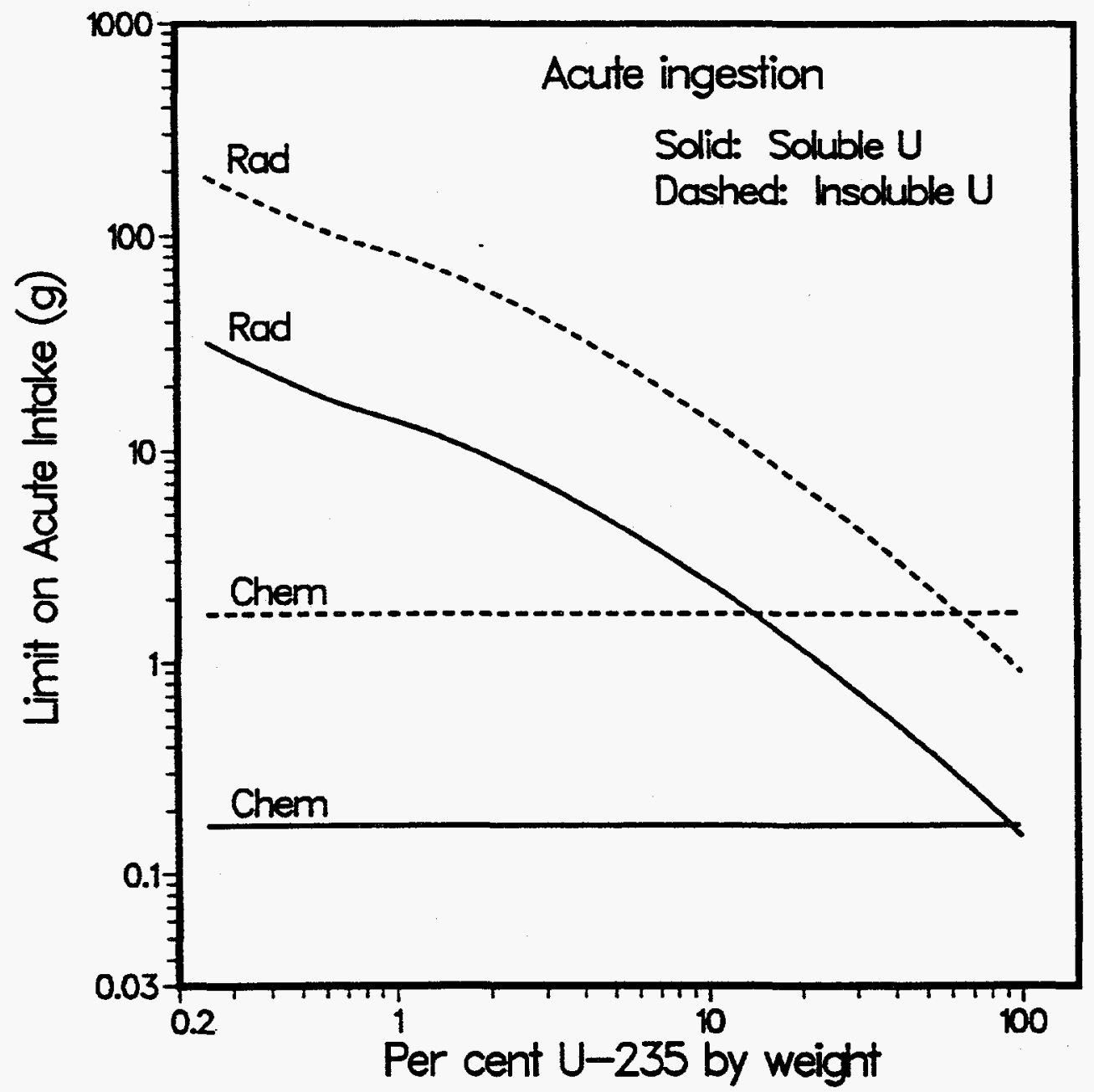

Fig. D.5. Comparison of limits on acute ingestion of uranium based on radiological (Rad) and chemical (Chem) guidance for different isotopic mixtures and solubility levels. 


\section{D.6 REFERENCES}

Foulkes, E. C. The concept of critical levels of toxic heavy metals in target tissues. Crit. Rev. Toxicol. 20:327-339; 1990.

International Commission on Radiological Protection. Report of Committee 2 on permissible dose for internal radiation. Oxford: Pergamon Press; ICRP Publication 2; 1959.

International Commission on Radiological Protection. Limits for intakes of radionuclides by workers. Oxford: Pergamon Press; Publication 30, Part 1; 1979.

International Commission on Radiological Protection. Human respiratory tract model for radiological protection. Pergamon Press, Oxford. 1994.

International Commission on Radiological Protection. Age-dependent doses to members of the public from intake of radionuclides, Pergamon Press, Oxford. Part 3: Publication 69; 1995.

International Commission on Radiological Protection. 1990 recommendations of the ICRP. Oxford: Pergamon Press. Publication 60; 1991.

Leggett, R. W. Basis for the ICRP's age-specific biokinetic model for uranium. Health Physics 67:589$610 ; 1994$.

Leggett, R. W. The behavior and chemical toxicity of uranium in the kidney: a reassessment. Health Phys. 57, 1989, 365-383.

Morrow, P. E. Biokinetics and toxicology of uranium. In: Moore, R. H., ed. Biokinetics and analysis of uranium in man. United States Uranium Registry, USUR-05, HEHF-47, 1984:E1-E27. Available from National Technical Information Service, 5285 Port Royal Road, Springfield, VA, 22151.

Morrow, P. E.; Gelein, R. M.; Beiter, H. D.; Scott, J. B.; Picano, J. J.; Yuile, C. L. Inhalation and intravenous studies of $\mathrm{UF}_{6} / \mathrm{UO}_{2} \mathrm{~F}_{2}$ in dogs. Health Phys. 43:859-873; 1982.

Rich, B. L.; Hinnefeld, S. L.; Lagerquist, C. L.; Mansfield, W. G.; Munson, L. H.; Wagner, E. R. Manual of good practices at uranium facilities - Draft. EGG-2530. Idaho Falls, Idaho: Idaho National Engineering Laboratory; 1988.

Spoor, N. L.; Hursh, J. B. Protection criteria. In: Hodge, H. C.; Stannard, J. N.; Hursh, J. B.; eds. Uranium, plutonium, transplutonic elements: handbook of experimental pharmacology, Vol. 36, Chap. 5. New York: Springer-Verlag; 1973:241-270.

Stopps, G. J.; Todd, M. The chemical toxicity of uranium with special reference to effects on the kidney and the use of urine for biological monitoring. Atomic Energy Control Board of Canada, 1982. Box 1046, Ottawa, K1P5S9; INFO-0074. 
Sula, M. J.; Carbaugh, E. H.; Bihl, D. E. Technical basis for internal dosimetry at Hanford. PNL-6866, UC-41. Richland, Washington: Pacific Northwest Laboratory; 1989.

SuLu, Zhao Fu-Yao. Nephrotoxic limit and annual limit on intake for natural U. Health Phys. 58:619$623 ; 1990$.

Voegtlin, C. and Hodge, H. C., eds. Pharmacology and toxicology of uranium compounds, National Nuclear Energy Series, Division VI - Volume I, Parts III and IV. New York: McGraw-Hill; 1953.

Wrenn, M. E.; Durbin, P. W.; Howard, B.; Lipsztein, J.; Rundo, J.; Still, E. T.; Willis, D. L. Metabolism of Ingested $U$ and Ra, Health Phys. 48:601-633; 1985. 
APPENDIX E

COMPARISON OF THE BIOKINETICS OF

TUNGSTEN AND URANIUM IN ADULT HUMANS

R. W. Leggett 



\section{APPENDIX E. COMPARISON OF THE BIOKINETICS OF TUNGSTEN AND URANIUM IN ADULT HUMANS}

Comparisons were made of the biokinetics of tungsten and uranium in adult humans, based on the biokinetic models described in this report. These models include the current respiratory and gastrointestinal tract models of the International Commission on Radiological Protection (Appendix C), a new systemic biokinetic model for tungsten (Appendix $\mathrm{C}$ ), and a recently developed systemic biokinetic model for uranium (Appendix $\mathrm{D}$ ). The following exposure cases were considered:

1. injection of tungsten and uranium into blood, corresponding roughly to the case of rapid absorption of these elements from a wound;

2. inhalation of relatively soluble, moderately soluble, or relatively insoluble forms of tungsten and uranium, assuming a particle size (activity median aerodynamic diameter or AMAD) of $5 \mu \mathrm{m}$;

3. ingestion of relatively soluble forms of tungsten and uranium.

Acute rather than chronic intakes were considered in all cases to facilitate comparison of the timedependent behaviors of these two elements in the body. The comparisons focused mainly on the timedependent retention of these elements in skeleton and kidneys. For the injection case, comparisons were also made for liver and for the collective remaining soft tissues.

It should be kept in mind that the systemic biokinetic model for uranium was based on a relatively large amount of experimental, occupational, and environmental data on humans as well as data on other species, while the model for tungsten was based entirely on animal data. Therefore, more confidence can be placed in the predictions for uranium than in those for tungsten.

Comparisons for the injection case (Figs. E.1 through E.4) indicate broadly similar behavior of tungsten and uranium atoms that reach the systemic circulation. The rate of urinary excretion is high for both elements, with the result that a relatively small portion of injected or absorbed material remains after one day.

Uranium in the form of the uranyl ion is expected to replace calcium at some locations in bone crystal, while tungsten in the form of tungstate is expected to replace phosphate. Similarities in the fate of calcium and phosphate in bone may result in broad similarities in the fates of uranium and tungsten in bone. Greater uptake of uranium than tungsten by bone (Fig. E.1) may result from greater competition for tungsten by the kidneys; that is, urinary clearance from plasma is greater for tungsten than for uranium, leaving less tungsten than uranium available for deposition in bone. The pattern of removal of tungsten from bone may differ slightly from that of uranium in the first few weeks after uptake, but long-term removal of both elements by bone is expected to be determined by bone turnover rates. Thus, the bone retention curves for tungsten and uranium should be nearly parallel after a few weeks or months (Fig. E.1).

Results of animal studies indicate an initially high concentration of uranium in the kidneys due to attachment of filtered uranium to the walls of the renal tubules. Animal studies also show an elevated 


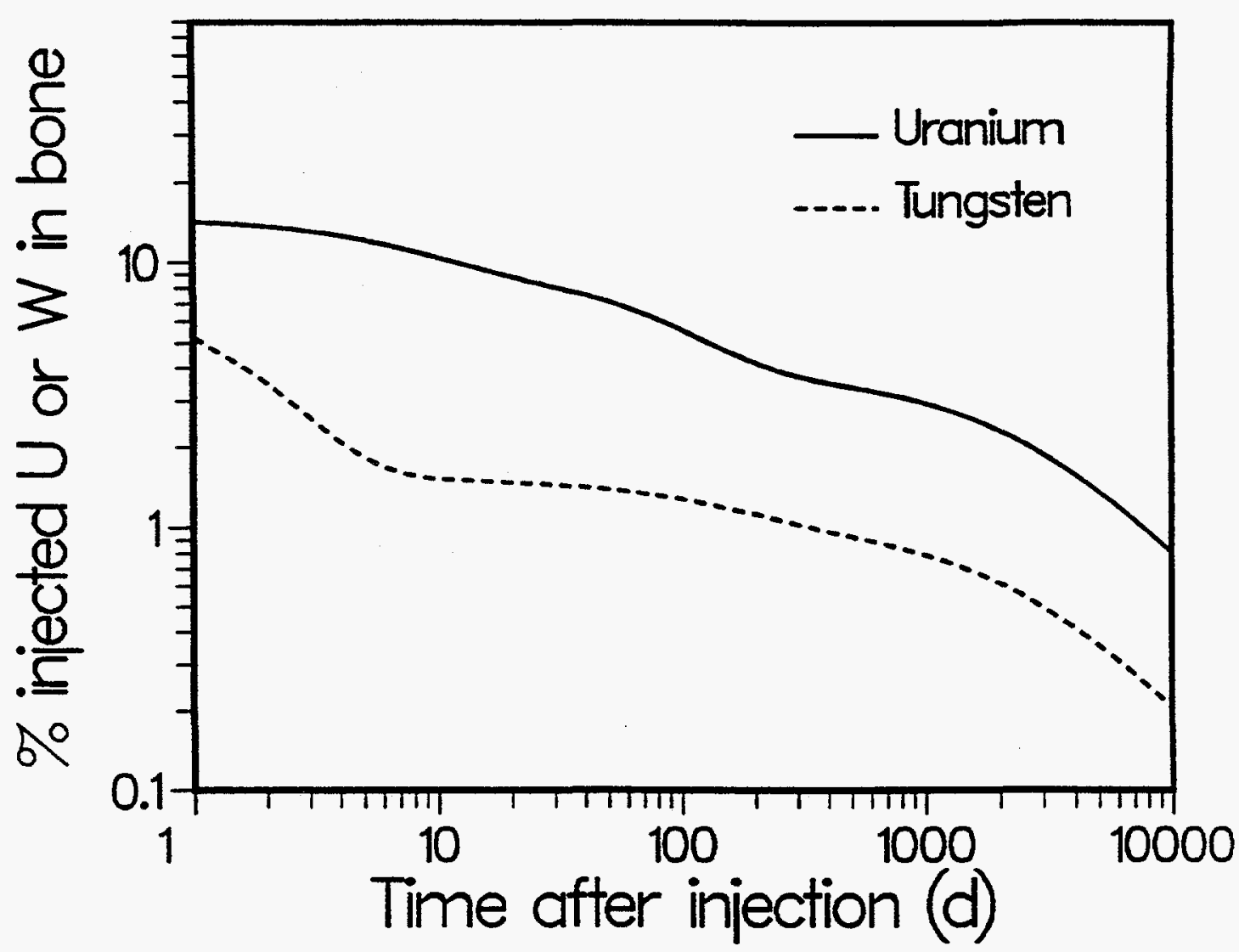

Fig. E.1. Comparison of content of tungsten and uranium in the skeleton of an adult human as a function of time after injection (e.g., puncture wound, with rapid absorption to blood), as predicted by the biokinetic models used in this report.

concentration of tungsten in the kidneys at early times but lower than that observed for uranium (Fig. E.2). Within a few days after uptake to blood, a major portion of uranium deposited in the renal tubules is removed to the tubular lumen and is subsequently removed in urine. The smaller amount of tungsten deposited in the kidneys may be lost more slowly, resulting in temporarily greater retention of tungsten than uranium in the kidneys by a few weeks after injection (Fig. E.2).

The models predict that the concentration in kidneys, liver, and remaining tissues declines faster for tungsten than for uranium at times remote from injection (Figs. E.2 through E.4). Predictions concerning long-term retention of tungsten in soft tissues are highly uncertain, however, because the underlying data are sparse and physiological considerations are of little help in this case. By contrast, there is reasonably strong evidence that a small portion of absorbed uranium is tenaciously retained in soft tissues.

Predictions of the percent of intake of tungsten and uranium in bone and kidneys as a function of time after inhalation of these elements are shown in Figs. E.5 through E.10. Predictions for Type F material are shown in Figs. E.5 and E.6, for Type $M$ material in Figs. E.7 and E.8, and for Type $S$ material in Figs. E.9 and E.10. The retention curves for bone and kidneys in Figs. E.5 through E.10 differ in magnitude from those for the injection case (Figs. E.1 and E.2) because of the decreased fractions of 


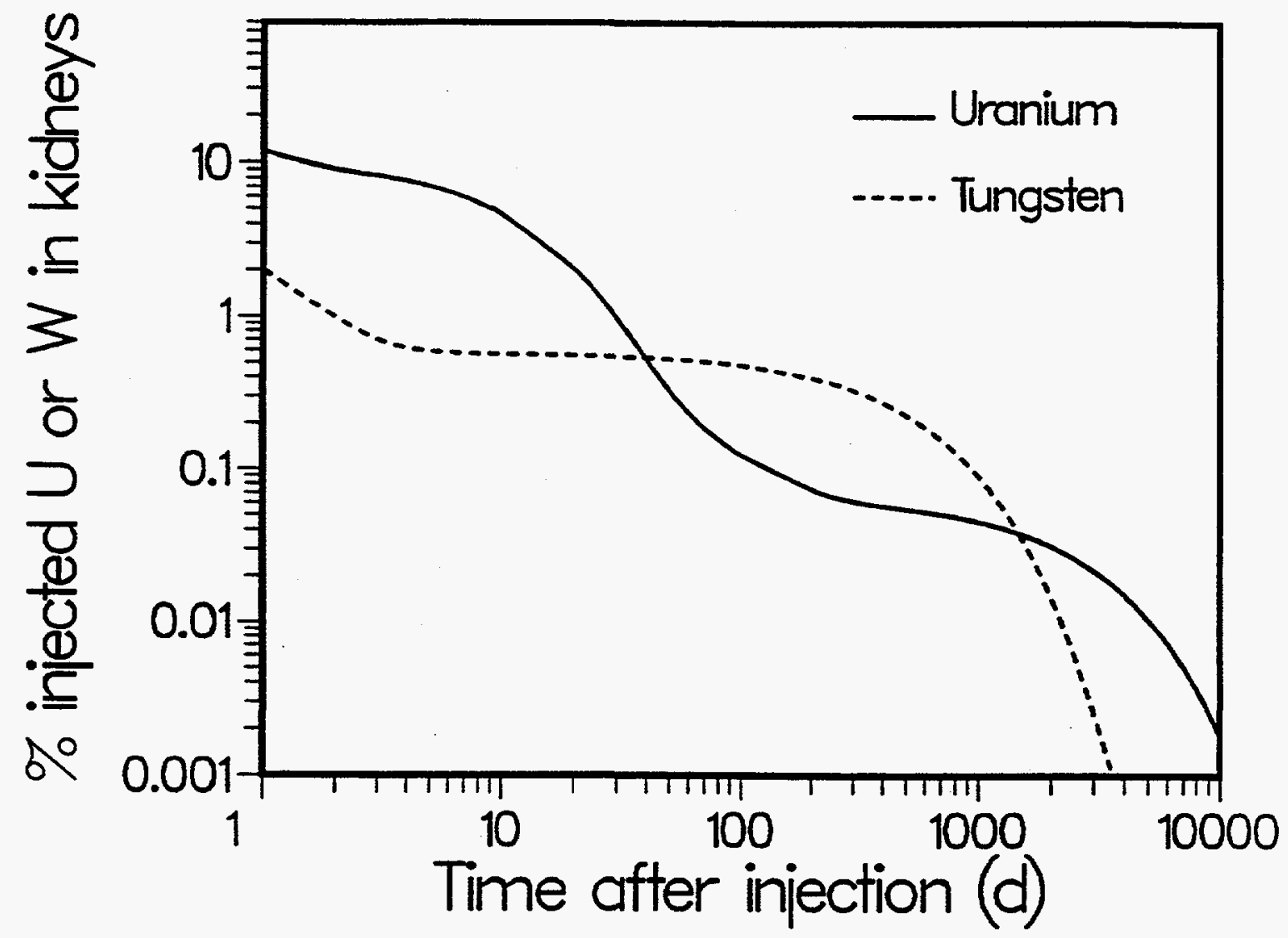

Fig. E.2. Comparison of content of tungsten and uranium in kidneys of an adult human as a function of time after injection (e.g., puncture wound, with rapid absorption to blood), as predicted by the biokinetic models used in this report.

intake reaching blood in the case of inhalation. In addition, the retention curves for the cases of inhalation of Type $M$ and $S$ material differ in shape to some extent from the curves for the injection case due to a delay in absorption of material from the lungs to blood.

For a particle size of $5 \mu \mathrm{m}$ (AMAD), the respiratory tract model predicts that the fraction of inhaled material that is eventually absorbed to blood is approximately $0.28+\left(0.2 \times f_{1}\right)$ for Type $F, 0.06+$ $\left(0.42 \times f_{1}\right)$ for Type $M$, and $0.007+\left(0.48 \times f_{1}\right)$ for Type $S$, where $f_{1}$ is the absorption fraction for material moving from the lungs to the GI tract. An $f_{1}$ value of 0.5 was used here for tungsten of Type $F$ or $M$, and a value of 0.02 was used for uranium of Type $F$ or $M$. The $f_{1}$ value for Type $S$ was assumed to be $10 \%$ of the value for Type $F$ or $M$, i.e., 0.05 for tungsten and 0.002 for uranium. Based on these $f_{1}$ values, the fractions of inhaled tungsten and uranium that eventually reach blood are, respectively, 0.38 and 0.28 for Type F, 0.27 and 0.07 for Type M, and 0.03 and 0.008 for Type S. With regard to relative amounts of tungsten and uranium that would be accumulated in systemic tissues, the net result is that the greater urinary excretion rate for tungsten would be offset to some extent by a greater fractional absorption of inhaled tungsten to blood, particularly for Type $M$ and Type S materials. Also, for Type $\mathrm{M}$ and $\mathrm{S}$ materials, a substantial portion of tungsten that reaches blood would enter via absorption from the GI tract and would enter the systemic circulation earlier than uranium, which would be absorbed mainly from the lungs. 


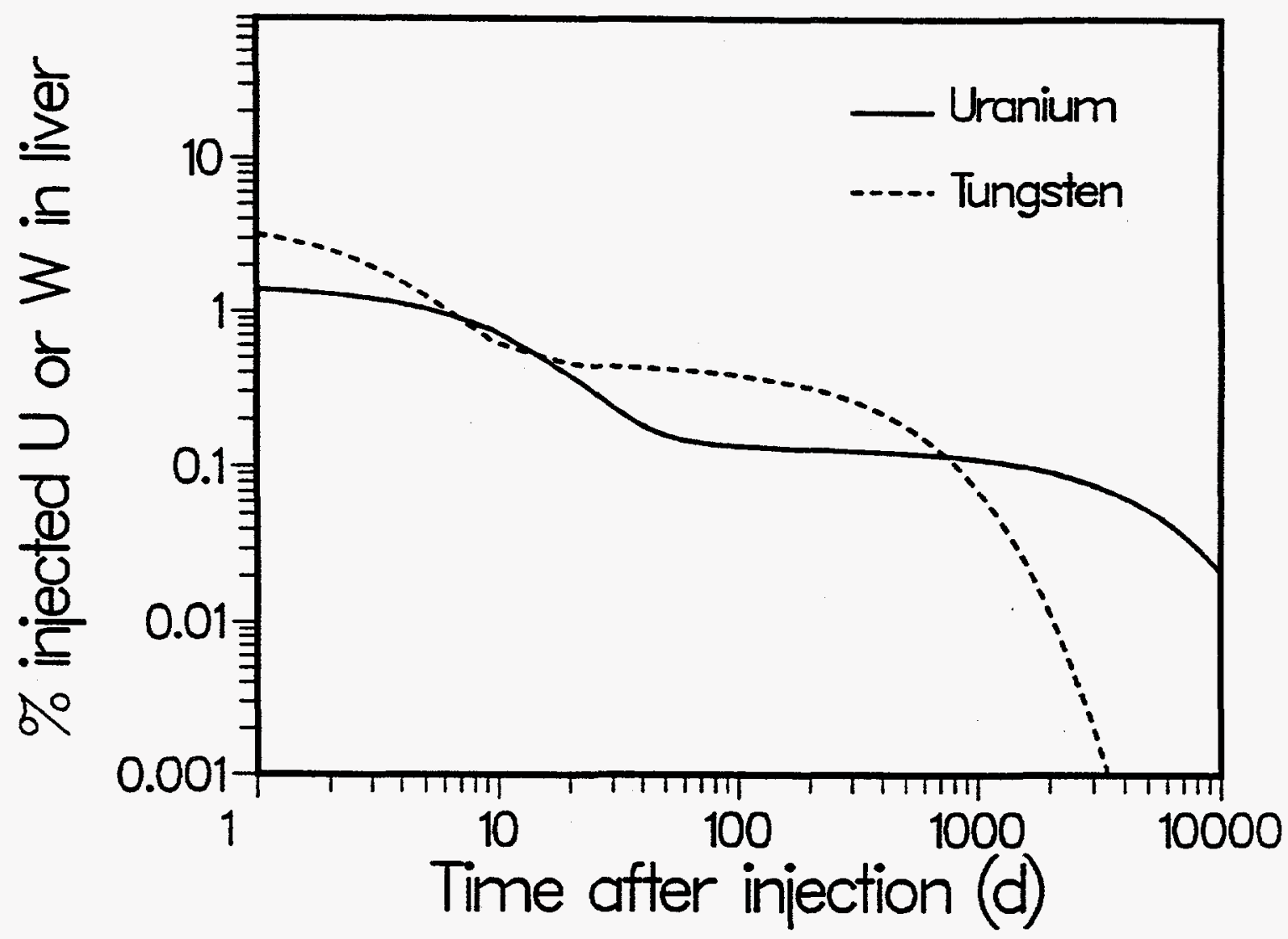

Fig. E.3. Comparison of content of tungsten and uranium in the liver of an adult human as a function of time after injection (e.g., puncture wound, with rapid absorption to blood), as predicted by the biokinetic models used in this report.

In the case of ingestion of soluble tungsten and uranium (Figs. E.11 and E.12), the amount (percent of intake) of tungsten retained in systemic tissues is predicted to be much greater than that of uranium at early times due to the 25 -fold higher GI uptake fraction assumed for tungsten than uranium (0.5 versus 0.02 ). Because absorption from the GI tract is assumed to occur in the first few hours after ingestion, the shapes of the retention curves for bone and kidneys (Figs. E.11 and E.12, respectively) are the same as those predicted for the injection case (Figs. E.1 and E.2, respectively). 


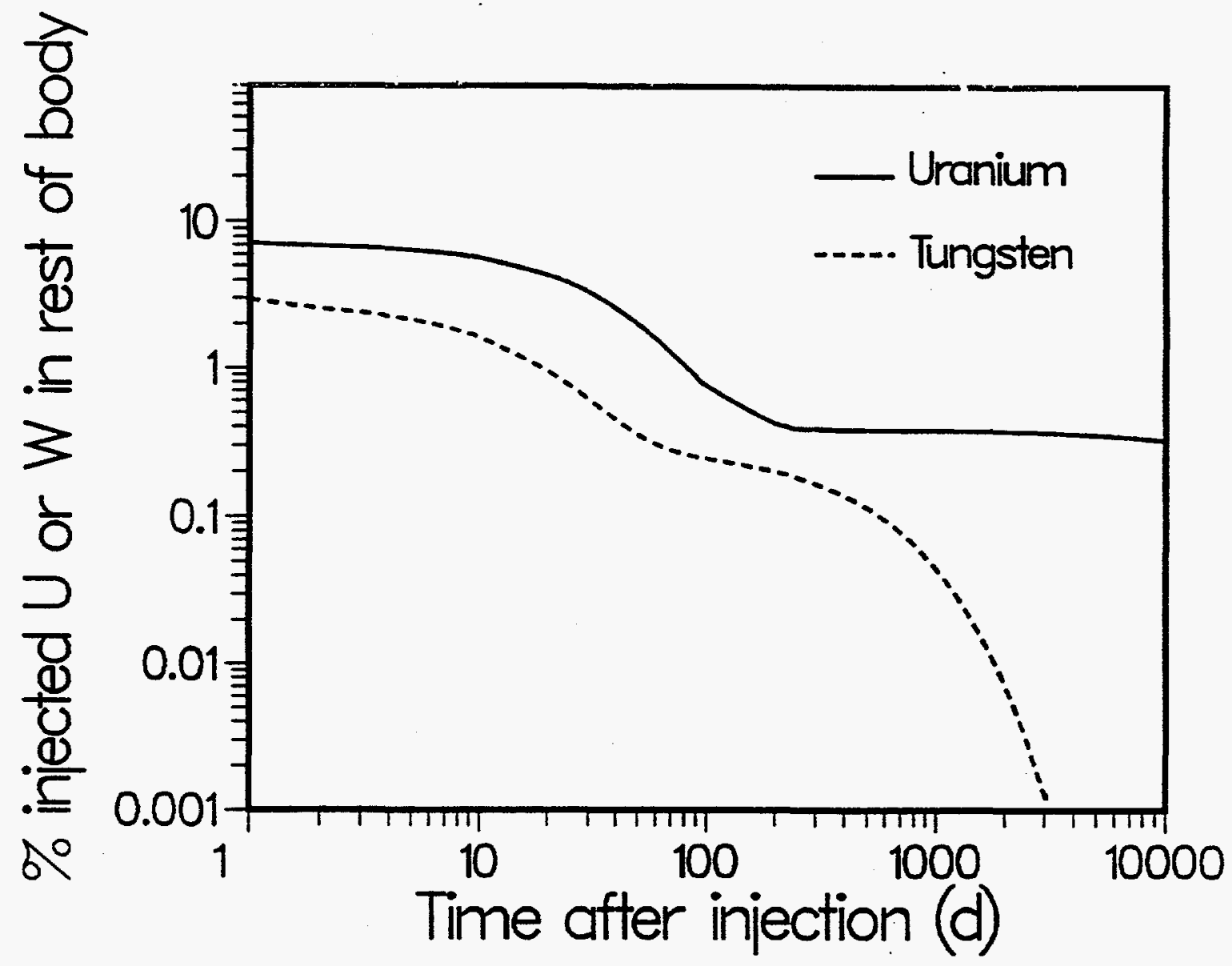

Fig. E.4. Comparison of content of tungsten and uranium in other soft tissues of an adult human as a function of time after injection (e.g., puncture wound, with rapid absorption to blood), as predicted by the biokinetic models used in this report. 


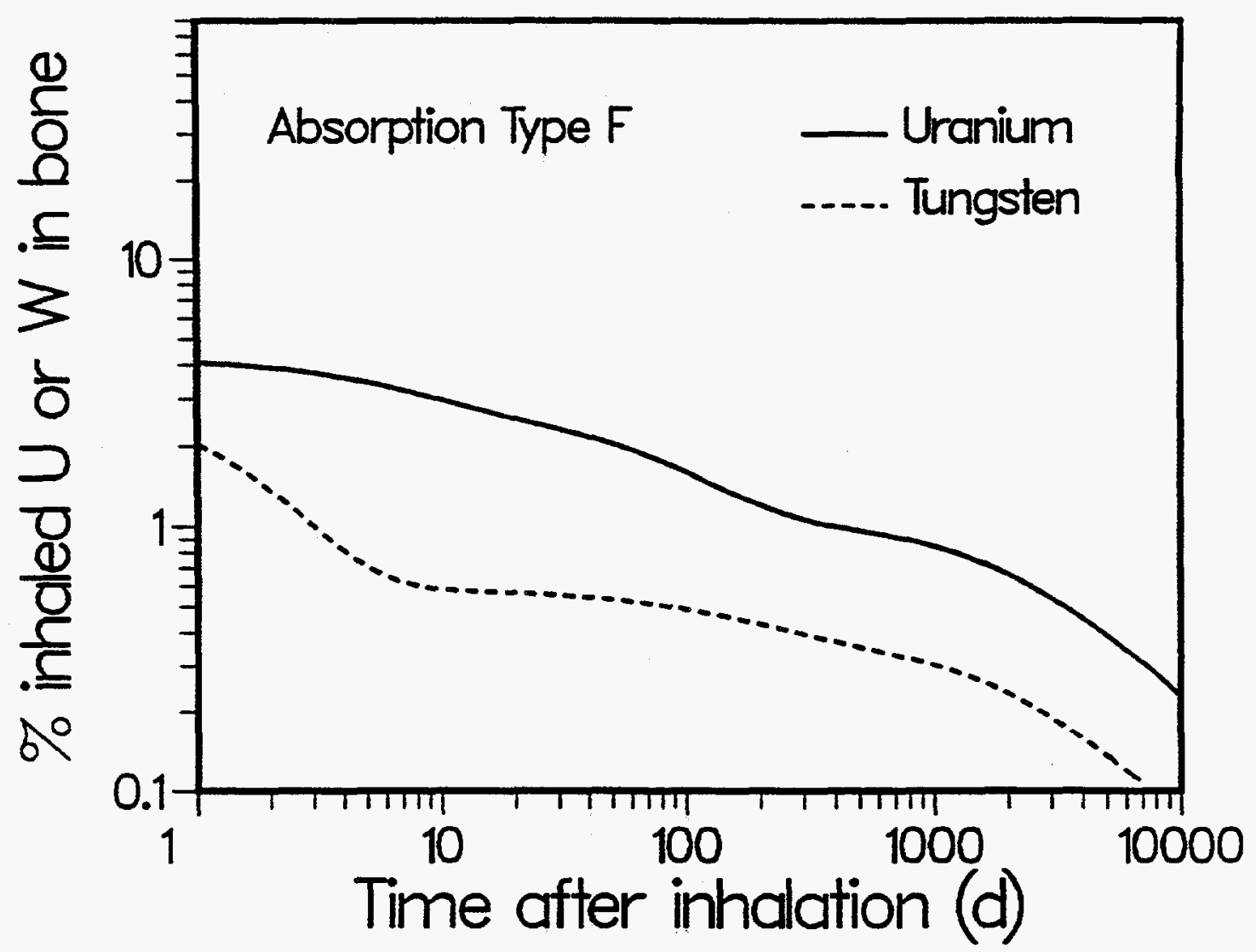

Fig. E.5. Comparison of content of tungsten and uranium in the skeleton of an adult human as a function of time after inhalation of type $F$ material (fast absorption to blood), as predicted by the biokinetic models used in this report. A particle size of $5 \mu \mathrm{m}$ (activity median aerodynamic diameter) is assumed. 


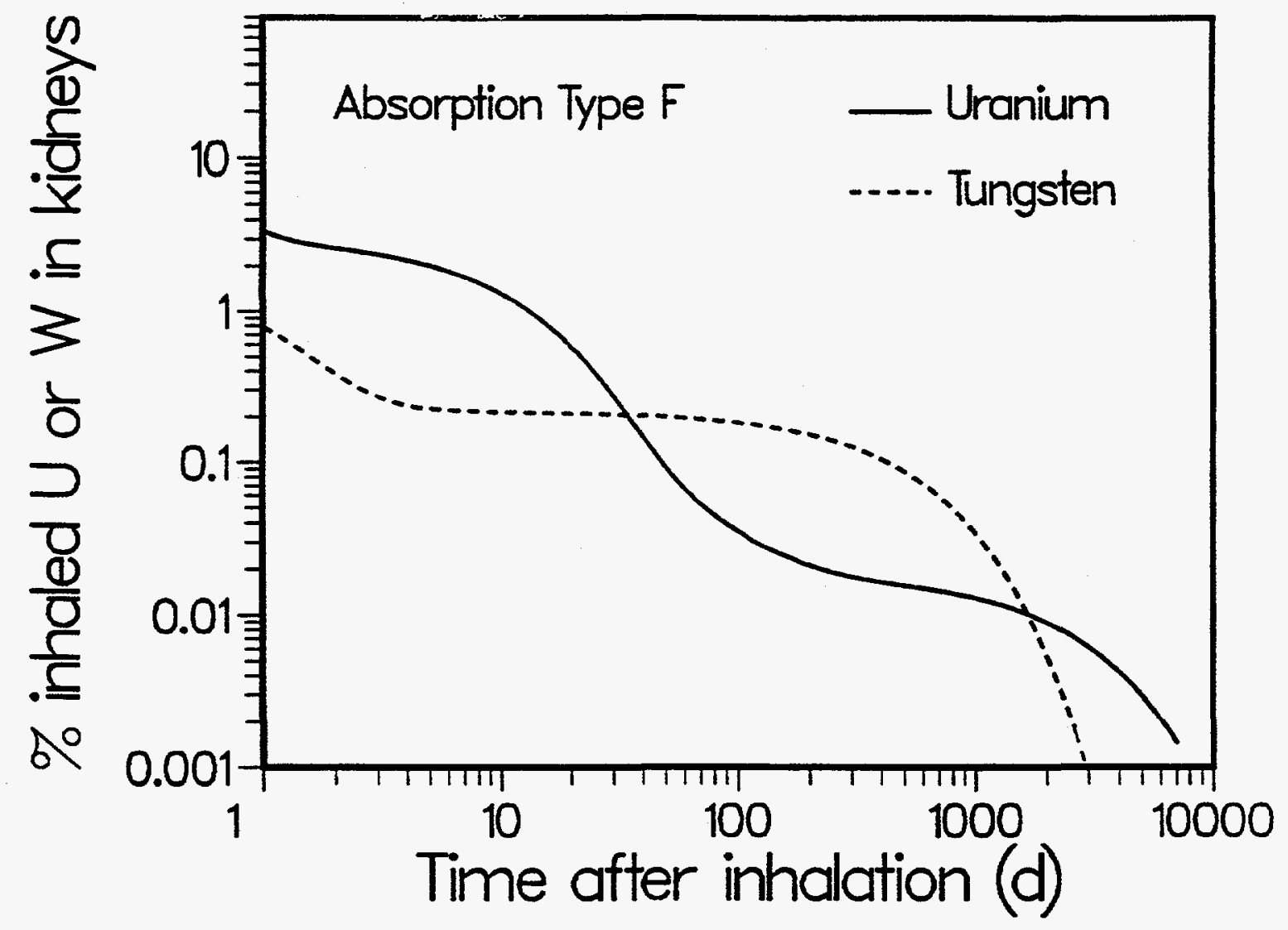

Fig. E.6. Comparison of content of tungsten and uranium in the kidneys of an adult human as a function of time after inhalation of type $\mathbf{F}$ material (fast absorption to blood), as predicted by the biokinetic models used in this report. A particle size of $5 \mu \mathrm{m}$ (activity median aerodynamic diameter) is assumed. 


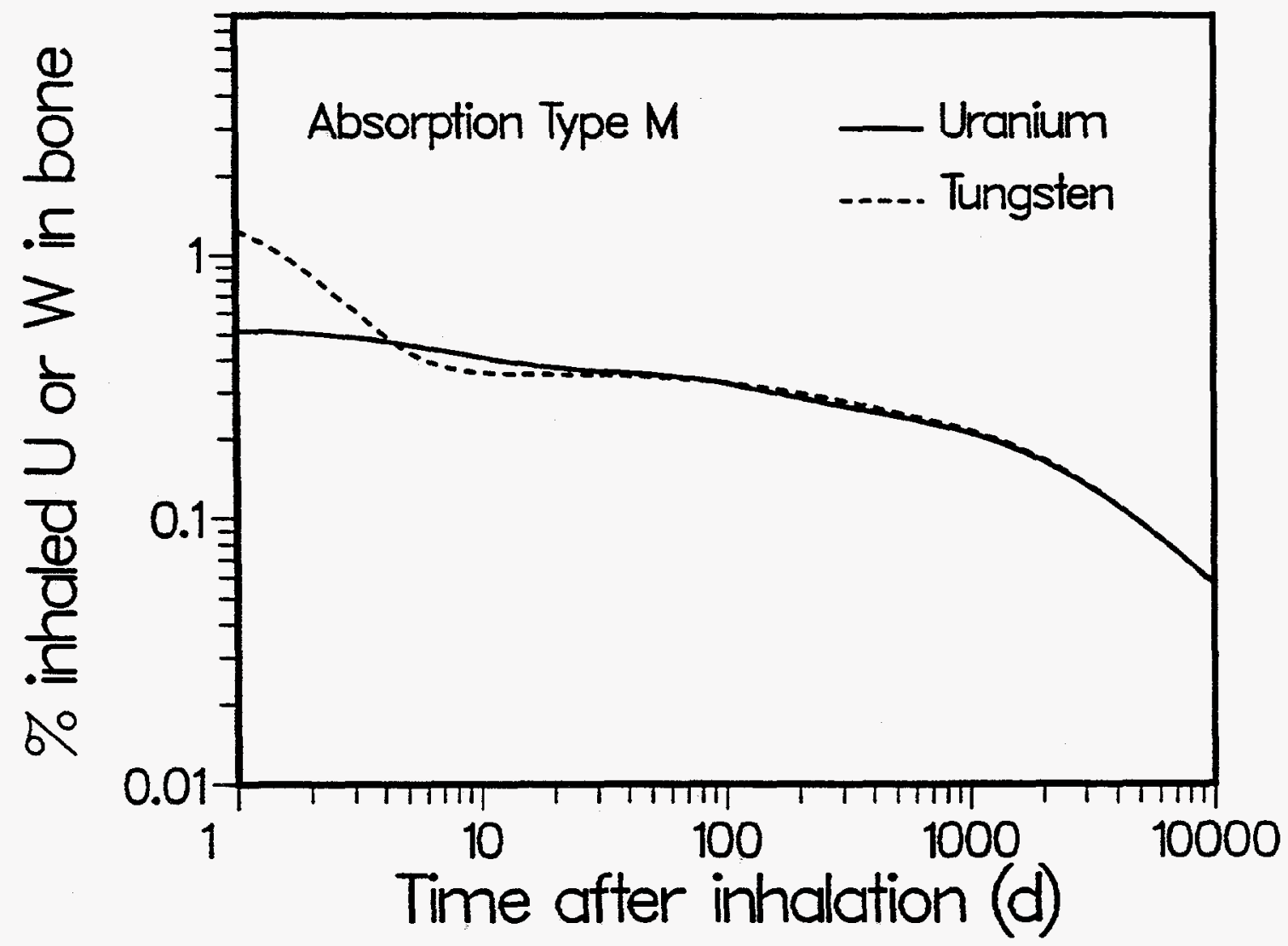

Fig. E.7. Comparison of content of tungsten and uranium in the skeleton of an adult human as a function of time after inhalation of type $M$ material (moderate absorption to blood), as predicted by the biokinetic models used in this report. A particle size of $5 \mu \mathrm{m}$ (activity median aerodynamic diameter) is assumed. 


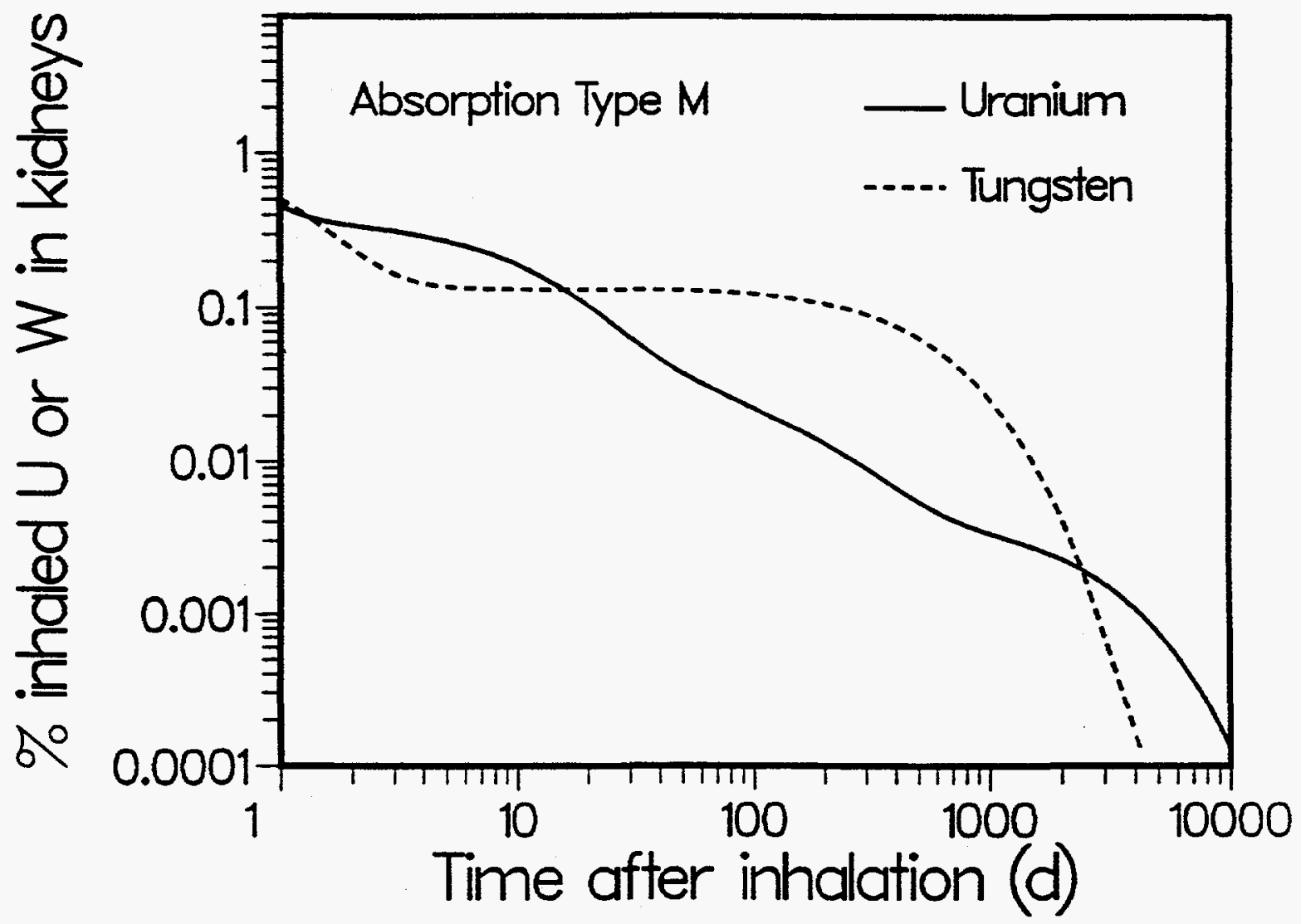

Fig. E.8. Comparison of content of tungsten and uranium in the kidneys of an adult human as a function of time after inhalation of type $M$ material (moderate absorption to blood), as predicted by the biokinetic models used in this report. A particle size of $5 \mu \mathrm{m}$ (activity median aerodynamic diameter) is assumed. 


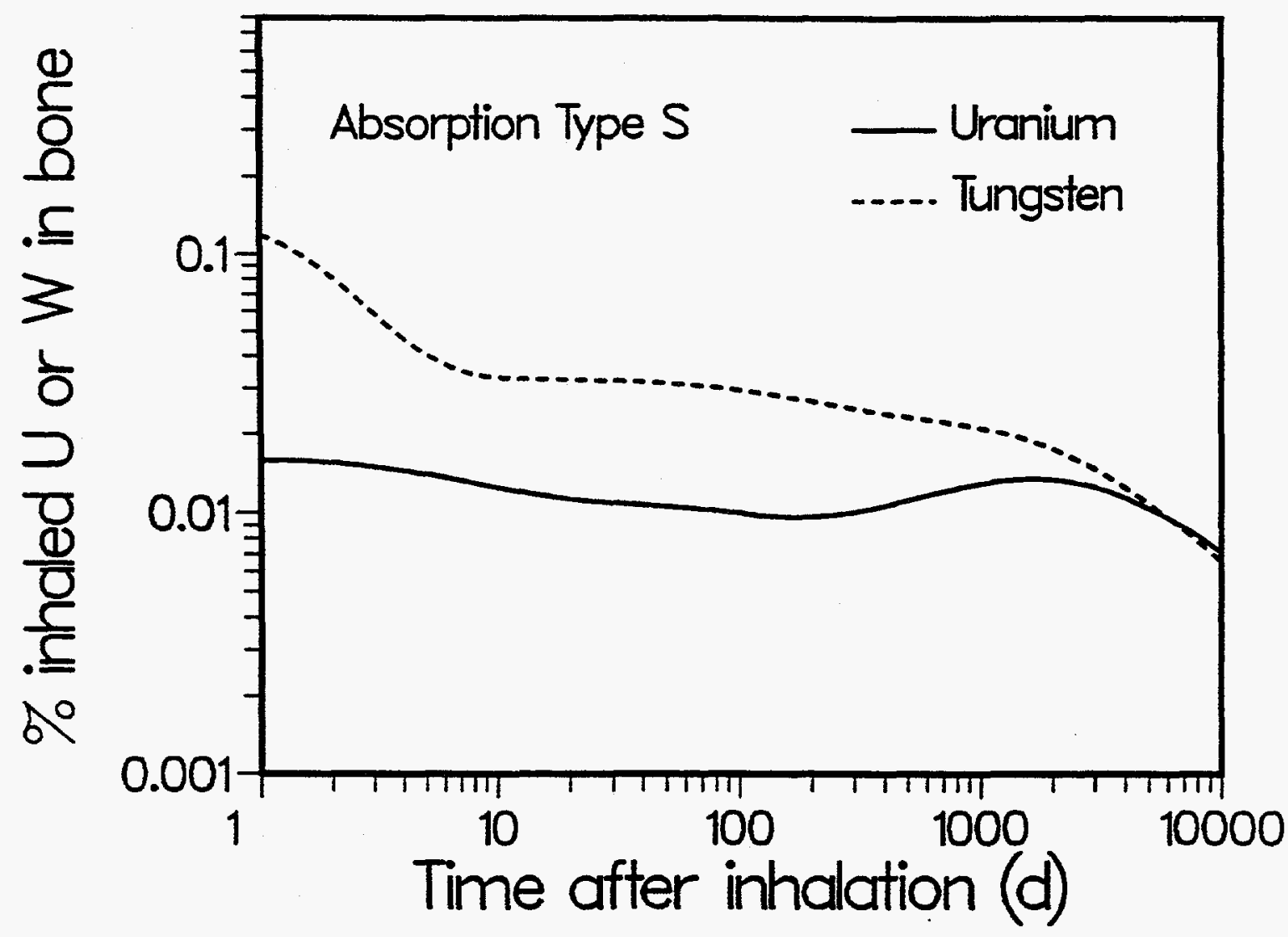

Fig. E.9. Comparison of content of tungsten and uranium in the skeleton of an adult human as a function of time after inhalation of type $S$ material (slow absorption to blood), as predicted by the biokinetic models used in this report. A particle size of $5 \mu \mathrm{m}$ (activity median aerodynamic diameter) is assumed. 


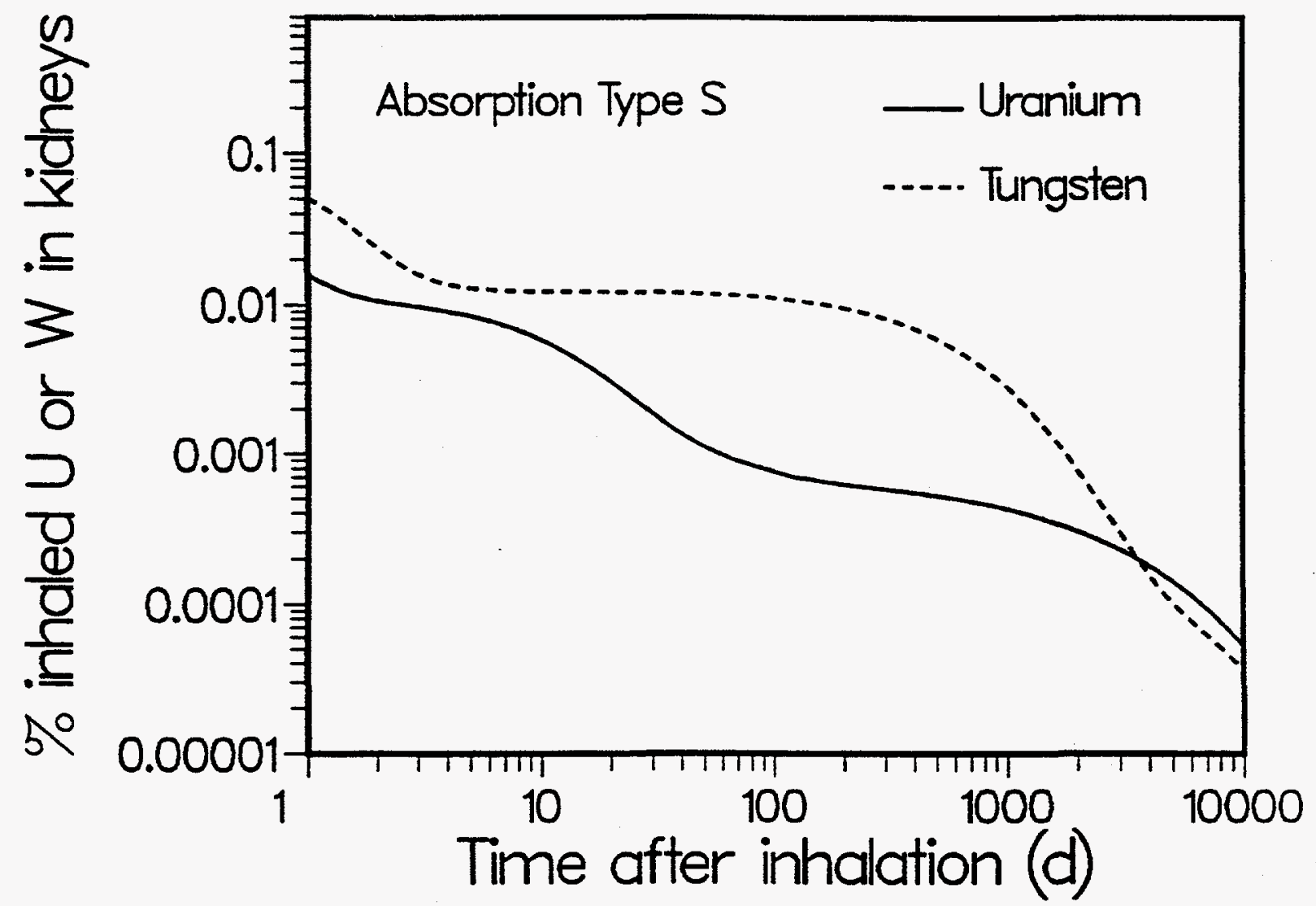

Fig. E.10. Comparison of content of tungsten and uranium in the kidneys of an adult human as a function of time after inhalation of type $S$ material (slow absorption to blood), as predicted by the biokinetic models used in this report. A particle size of $5 \mu \mathrm{m}$ (activity median aerodynamic diameter) is assumed. 


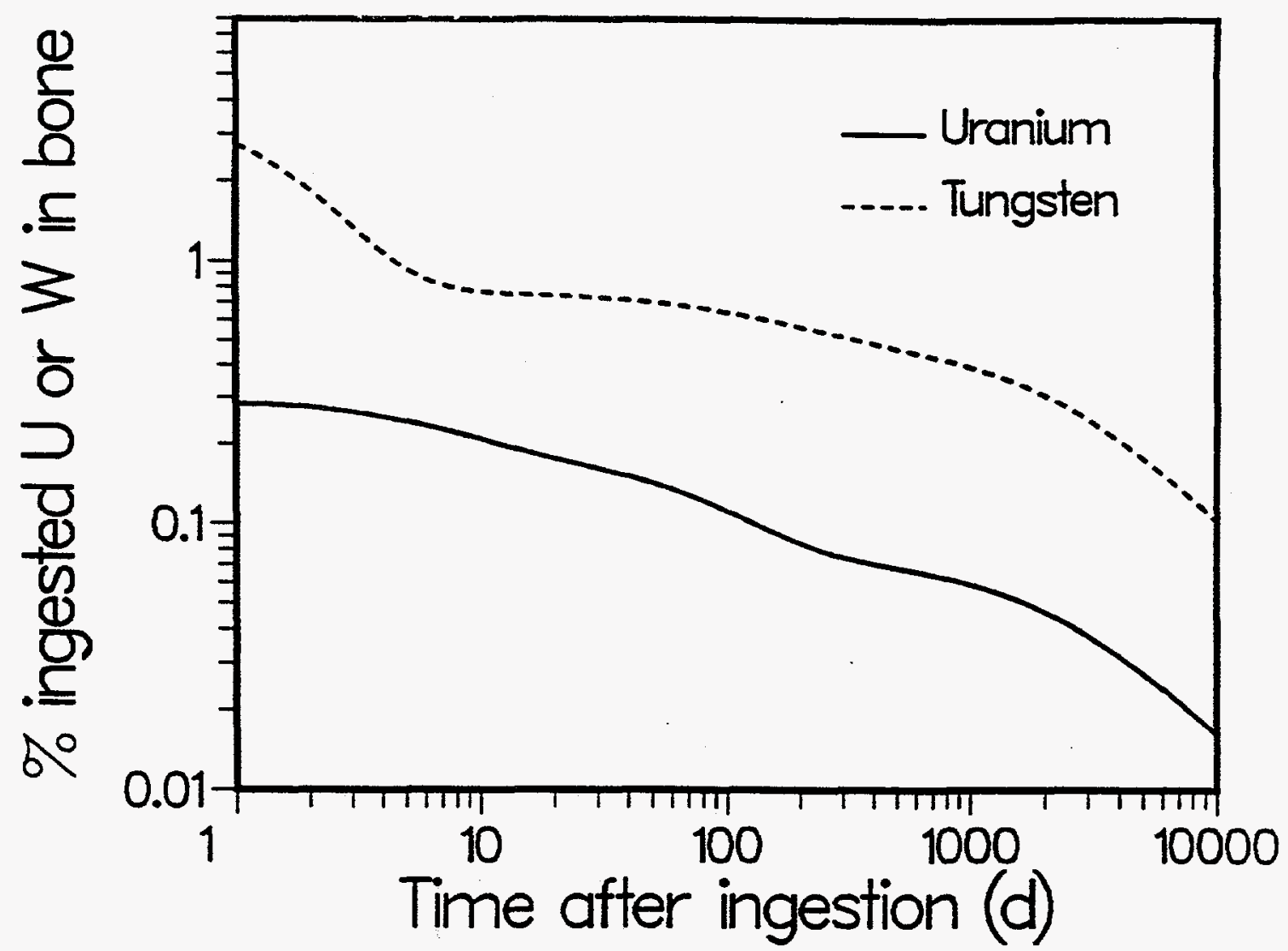

Fig. E.11. Comparison of content $f$ tungsten and uranium in the skeleton of an adult human as a function of time after ingestion of soluble material, as predicted by the biokinetic models used in this report. 


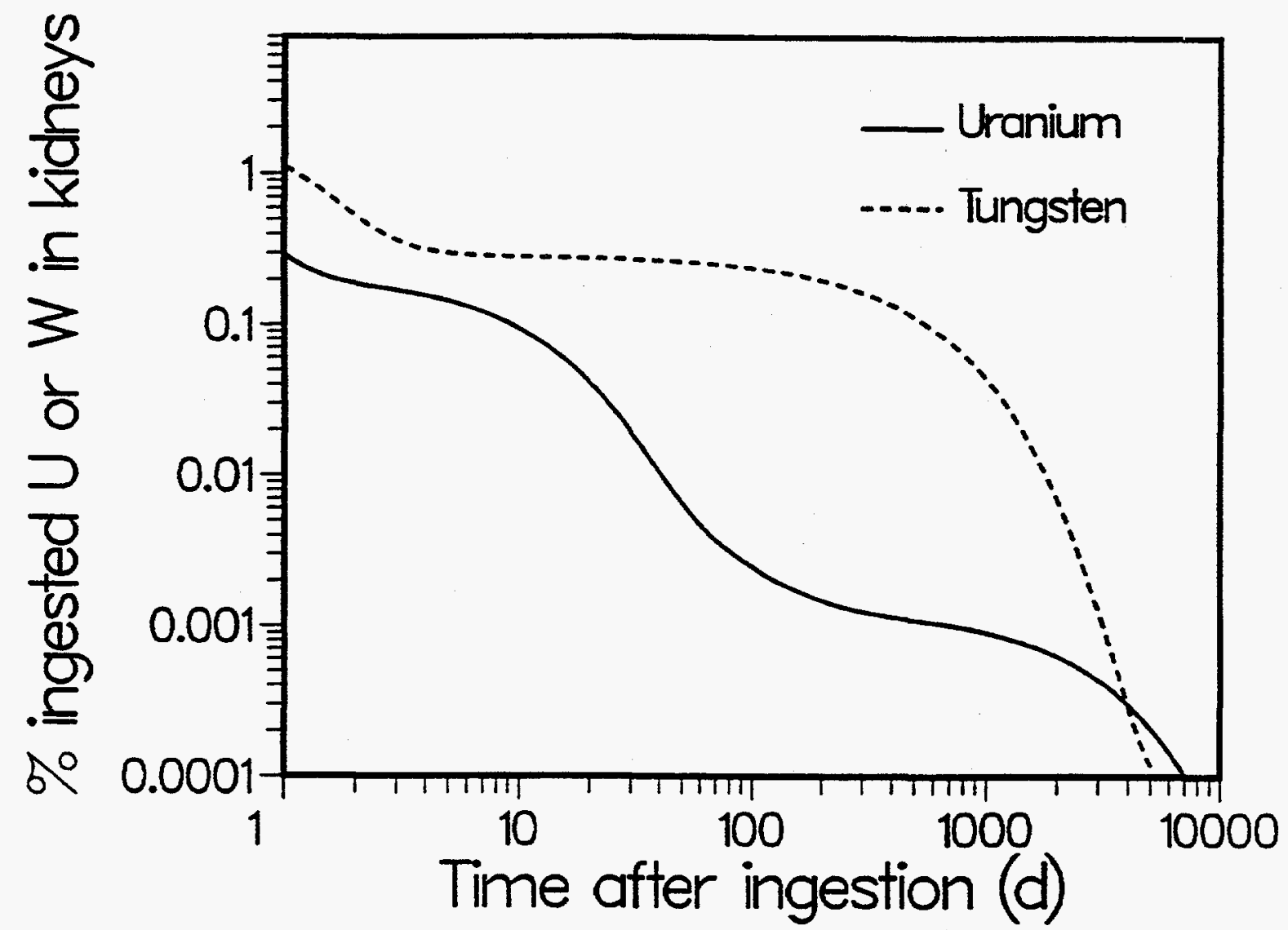

Fig. E.12. Comparison of content of tungsten and uranium in the kidneys of an adult human as a function of time after injection of soluble material, as predicted by the biokinetic models used in this report. 

APPENDIX F

NONCANCER HEALTH EFFECTS

N. B. Munro and E. K. Bowman 



\section{APPENDIX F. NONCANCER HEALTH EFFECTS}

\section{F.1 ACUTE TOXICITY}

\section{F.1.1 Workplace Standards}

Available information on the regulations and guidelines for workplace exposures in the U.S.A. to tungsten is listed in Table F.1. The permitted levels reflect the low acute toxicity of tungsten and the low uptake of insoluble forms, as well as an assumption of low chronic toxicity. The $5 \mathrm{mg} / \mathrm{m}^{3}$ guideline for insoluble forms is, in fact, equivalent to that for nuisance dust.

Table F.1. Regulations and guidelines for workplace exposures to soluble and insoluble forms of tungsten

\begin{tabular}{|c|c|c|c|}
\hline Agency & Description & $\begin{array}{l}\text { Tungsten, soluble } \\
\text { forms, } \mathrm{mg} / \mathrm{m}^{3}\end{array}$ & $\begin{array}{l}\text { Tungsten, insoluble } \\
\text { forms, } \mathrm{mg} / \mathrm{m}^{3}\end{array}$ \\
\hline OSHA & $\begin{array}{l}\text { TLV (contaminants in } \\
\text { construction, shipyards) }\end{array}$ & 1 & 5 \\
\hline ACGIH & $\begin{array}{l}\text { TLV-8 hr TWA } \\
\text { TLV-STEL }\end{array}$ & $\begin{array}{l}1 \\
3\end{array}$ & $\begin{array}{l}5 \\
10\end{array}$ \\
\hline NIOSH & $\begin{array}{l}\text { Recommended exposure limit; } \\
\text { 15-min exposure limit }\end{array}$ & $\begin{array}{l}\text { NA } \\
\text { NA }\end{array}$ & $\begin{array}{l}5 \\
10\end{array}$ \\
\hline NIOSH & IDLH concentration ${ }^{d}$ & None established & None established \\
\hline
\end{tabular}

${ }^{a}$ OSHA 1995.

${ }^{b}$ ACGIH 1994.

'ACGIH 1986.

IDLH = immediately dangerous to life or health (Ludwig, Cairelli, and Whalen 1994).

\section{F.1.2 Description of Acute Effects}

Table F.2 contains a summary of acute toxicity data from animal testing for four tungsten compounds. These data illustrate the relatively low acute toxicity of the more soluble tungsten compounds in animals. It is easy to see why tungsten has been viewed as relatively inert and assumptions might be made regarding low chronic toxicity. However, for purposes of this analysis, no assumptions were made and the literature was reviewed critically to see if it supports such a view. It should be born in mind that the approach to toxicology has changed considerably over the past 25 or 30 years from an emphasis on overt acute toxicity to more concern for more subtle chronic effects. Of chronic toxicity endpoints, an appreciation has been developing in the past several years that carcinogenicity may not be the most sensitive endpoint, but rather reproductive and developmental toxicity endpoints and neurotoxicity may be significantly more sensitive for at least some classes of toxicants. 
Table F.2. Acute toxicity data for several tungsten compounds

\begin{tabular}{lclll}
\hline Species & $\begin{array}{c}\text { Tungsten oxide, } \\
\text { oral } \mathrm{LD}_{50}, \mathrm{mg} / \mathrm{kg}\end{array}$ & $\begin{array}{l}\text { Sodium tungstate } \\
\text { oral } \mathrm{LD}_{50}, \mathrm{mg} / \mathrm{kg}\end{array}$ & $\begin{array}{c}\text { Ammonium-p-tungstate, } \\
\text { oral } \mathrm{LD}_{50}, \mathrm{mg} / \mathrm{kg}\end{array}$ & $\begin{array}{c}\text { Sodium } \\
\text { phosphotungstate, } \\
\text { oral } \mathrm{LD}_{50}, \mathrm{mg} / \mathrm{kg}\end{array}$ \\
\hline Mouse & $\mathrm{NA}^{c}$ & $240 \pm 13.5^{b}$ & $\mathrm{NA}$ & $700.0 \pm 79^{b}$ \\
Rat & $840^{b, c}$ & $1190 \pm 129.5^{b}$ & $11,300^{c}$ & $1600.0 \pm 201^{b}$ \\
Rabbit & $\mathrm{NA}$ & $875^{b}$ & $\mathrm{NA}$ & $\mathrm{NA}$ \\
Guinea pig & $\mathrm{NA}$ & $1152^{b}$ & $\mathrm{NA}$ & $\mathrm{NA}$ \\
\hline
\end{tabular}

${ }^{a}$ Abbreviations: $\mathrm{LD}_{50}=$ dose of substance causing death in $50 \%$ of population; $\mathrm{NA}=$ not available.

'Nadeenko 1966.

RTECS (April 1994): AIHA J. 30:470 (1969).

${ }^{d}$ Increase in dose up to $2500 \mathrm{mg} / \mathrm{kg}$ did not increase the percentage of deaths; RTECs (April 1994)

lists oral $\mathrm{LD}_{50}$ as $1059 \mathrm{mg} / \mathrm{kg}$, based on data reported in Nadeenko (1966).

\section{F.2. CHRONIC TOXICITY}

The goal of this section is to characterize the potential noncarcinogenic impacts on people as a result of exposure to tungsten. There will not be any simulations in this Appendix, only an attempt to define cause and effect. The most that could be expected is to say that a body burden of $\mathrm{x} \mu \mathrm{g} / \mathrm{kg}$ of tungsten has no effect and that above this threshold, the dose response function is described by some function $f(x)$. Realistically, we find that the data set available in the literature is very sparse. Thus we will probably not be able to give final answers for almost any biological endpoint that we are investigating. Our investigations include both cancer and non-cancer endpoints. The cancer endpoint is discussed in Appendix G. The non-cancer endpoints dealt with in some detail are neurotoxicity, reproductive toxicity, developmental toxicity, effects on glucose metabolism and storage, and inhibition of the enzymes xanthine oxidase and sulfite oxidase.

Tungsten compounds and tungsten mixture alloys are strongly implicated by animal experiments and human experience with pulmonary disease, disability, and in the case of hard metal disease, death. The evidence has been reviewed in NIOSH 1977 and Danisi 1991. Recent animal experiments show that tungsten particles and insoluble calcium tungstate powder are stimuli for a strong inflammatory response in the lung (Grande et al 1990; Aguas, Grande and Carvalho 1991; Pereira and Grande 1992; Peao, Aguas, and Grande 1992; Peao et al 1993). However, in view of the implication of the role of cobalt in most of the human experience, the pulmonary disease endpoint is not considered further in this review. Yet it should be kept in mind that recent in vitro experiments with macrophages point to enhanced toxicity from the combination of cobalt and tungsten carbide in hard metal, not just from cobalt or tungsten (Lison and Lauwerys 1990, 1991, 1992, 1993, 1994). Thus, eventually the combinations of tungsten with alloy candidates will need to be considered with respect to combined toxicity.

Table F. 3 contains a partial listing of various chronic effects observed in animal experimentation. This review focuses on those endpoints of more concern for possible human health effects from long-term, low-dose exposure, primarily from ingestion exposure. Three sensitive endpoints of most concern have been identified. Neurotoxicity is identified from the animal literature by disturbances of conditioned 
Table F.3. Partial list of chronic toxicity data for tungsten

\begin{tabular}{|c|c|c|c|c|c|c|}
\hline $\begin{array}{l}\text { Species/ } \\
\text { compound }\end{array}$ & Route & Dose & $\begin{array}{l}\text { Exposure } \\
\text { duration }\end{array}$ & $\begin{array}{l}\text { Tissue } \\
\text { level }\end{array}$ & Endpoints observed & Reference \\
\hline $\begin{array}{l}\text { Rat/Na } \\
\text { tungstate }\end{array}$ & $\mathrm{IP}^{a}$ & $0.5 \mu \mathrm{g}$ & $100 \mathrm{~d}$ & $\mathrm{NA}^{a}$ & $\begin{array}{l}\text { Brain, liver } \\
\text { glutaminase; brain } \\
\text { glutamic acid }\end{array}$ & $\begin{array}{l}\text { Capilna et } \\
\text { al } 1963\end{array}$ \\
\hline $\begin{array}{l}\text { Rats } / \mathrm{Na} \\
\text { tungstate }\end{array}$ & Per oral & $\begin{array}{l}100,50,25 \\
10 \mathrm{mg} / \mathrm{kg}\end{array}$ & $\begin{array}{l}\text { Subacute } \\
(? d)\end{array}$ & $\begin{array}{l}\text { Not } \\
\text { reported }\end{array}$ & Growth, blood $\mathrm{ChE}^{a}$ & $\begin{array}{l}\text { Nadeenko } \\
1966\end{array}$ \\
\hline $\begin{array}{l}\text { Rabbits } / \mathrm{Na} \\
\text { tungstate }\end{array}$ & Per oral & $\begin{array}{l}100,50,25 \\
10 \mathrm{mg} / \mathrm{kg}\end{array}$ & $\begin{array}{l}\text { Subacute } \\
(? d)\end{array}$ & $\begin{array}{l}\text { Not } \\
\text { reported }\end{array}$ & $\begin{array}{l}\text { Growth, } \mathrm{SH}^{a} \text { groups } \\
\text { in whole blood and } \\
\text { serum, glycogen- } \\
\text { forming system of } \\
\text { liver etc. }\end{array}$ & $\begin{array}{l}\text { Nadeenko } \\
1966\end{array}$ \\
\hline $\begin{array}{l}\text { Rats/Na } \\
\text { tungstate }\end{array}$ & Per oral & $\begin{array}{l}0.5,0.05 \\
0.005 \mathrm{mg} / \mathrm{kg}\end{array}$ & 7 month & $\begin{array}{l}\text { Measured } \\
\text { but not } \\
\text { reported }\end{array}$ & $\begin{array}{l}\text { Blood ChE, } \\
\text { conditioned reflexes; } \\
\text { SH groups of brain, } \\
\text { liver }\end{array}$ & $\begin{array}{l}\text { Nadeenko } \\
1966\end{array}$ \\
\hline $\begin{array}{l}\text { Rabbits/Na } \\
\text { tungstate }\end{array}$ & Per oral & $\begin{array}{l}5,0.5,0.05 \\
0.005 \mathrm{mg} / \mathrm{kg}\end{array}$ & 8 month & $\begin{array}{l}\text { Measured } \\
\text { but not } \\
\text { reported }\end{array}$ & $\begin{array}{l}\text { Blood alkaline } \\
\text { phosphatase; SH } \\
\text { groups, glycogen- } \\
\text { forming capacity of } \\
\text { liver; response to } \\
\text { galactose loading; } \\
\text { UGI microscopic } \\
\text { damage }\end{array}$ & $\begin{array}{l}\text { Nadeenko } \\
1966\end{array}$ \\
\hline
\end{tabular}

${ }^{a}$ Abbreviations: $\mathrm{IP}=$ intraperitoneal; $\mathrm{NA}=$ not available; $\mathrm{ChE}=$ cholinesterase; $\mathrm{SH}=$ sulfhydryl groups; UGI = upper gastrointestinal tract.

learning, reflex activity indices, brain histopathology, and decreases in blood cholinesterase. These effects have been obtained with chronic oral exposure to extremely low doses of sodium tungstate and with subchronic inhalation exposure to moderately low air concentrations (approximately $0.5 \mathrm{mg} / \mathrm{m} 3$ ). It is considered that this airborne concentration would be difficult to achieve in most environmental situations. Reproductive toxicity is indicated by observations of embryo death and also a sperm motility study. The latter used the same inhalation concentrations as above.

Effects on certain enzymes were also evaluated. We have obtained a perspective on xanthine and sulfite oxidase inhibition and glucose metabolism from a combination of literature data on tungsten and molybdenum for small mammals and other information on humans. At this time, very abnormal and unrealistic conditions appear to be required, in terms of trace element and/or sulfite concentrations in foods or water, before adverse effects due to sulfite oxidase inhibition could be induced.

Searches were performed, but no studies were located that dealt with effects on immune system function, especially from chronic low-dose exposure. It is not clear whether adequate observations have been made with modern techniques to rule out immunotoxicity, as negative data are seldom reported in the literature. 


\section{F.2.1 Neurotoxicity}

\section{F.2.1.1 Human Evidence}

Kaplun and Mezentseva (1959) evaluated health effects of exposure to dusts from the production of hard metal. Medical examination of a group of 247 workers exposed to mixed dusts containing tungsten and cobalt yielded 117 individuals with some damage to the upper respiratory tract; they complained of loss of appetite, nausea, and coughing. Of these 117 workers, a significant fraction (undefined) also had hypotension, unspecified blood changes, or an impaired sense of smell.

Vengerskaya and Salikhodzhaev (1962) also evaluated workers exposed to aerosols containing tungsten, cobalt and their compounds in a hard-metal plant. Eighty-eight out of 178 workers ( 52 men, 126 women) studied complained of dyspnea, coughing, pounding of the heart, headache, dizziness, nausea, loss of appetite, and impaired sense of smell. Tungsten was measured in blood and urine. Mean tungsten concentrations ranged from 0.8 to $1.1 \mathrm{mg} \%$ in 45 workers and $0.6-1.1 \mathrm{mg} / \mathrm{liter}$ in urine of 40 workers; it was not measurable in the blood of 11 subjects or in the urine of 7 subjects.

Several of the reported symptoms suggestive of neurotoxicity are common to both of these studies, including loss of appetite, nausea, and impaired sense of smell. Additionally, some workers studied by Vengerskaya and Salikhodzhaev (1962) reported dizziness and headache which also may be related to neurotoxicity.

Jordan et al (1990) evaluated a small group of 12 hard-metal workers having pulmonary problems and reporting memory deficits. They tested these individuals with the Wechsler Memory Scale-Revised and found statistically significant deficits in verbal memory $(P<0.001)$ and attention $(P<0.01)$, but no effect on visual-spatial memory or delayed memory. These workers had been exposed to hard metal consisting mainly of tungsten carbide and cobalt; the exposure had been both to dust and to a mist form with an unidentified organic solvent as vehicle.

\section{F.2.1.2 Animal Evidence}

Karantassis (1924) observed shortness of breath, loss of appetite, colic (gastrointestinal pain?), trembling, uncoordinated movements, and sudden jumps in guinea pigs exposed by gastric intubation or injection to acutely lethal doses of tungstate. The latter three signs are suggestive of neurotoxicity. Nadeenko (1966) also reported reactions suggestive of neurotoxicity in 300 albino mice and 40 white rats given acutely toxic doses of tungsten compounds. The animals showed a brief period of excitation, then a period of motor inhibition in which they sat with arched backs and displayed reduced motor tone. Some mice developed hind leg paralysis. These experiments were designed to determine the $\mathrm{LD}_{50}$ doses of sodium tungstate, sodium phosphotungstate, and tungstic anhydride $\left(\mathrm{WO}_{3}\right)$. The results of these and subsequently discussed studies giving evidence of neurotoxicity are presented in Table F.4.

In subacute experiments in albino rats and rabbits, Nadeenko (1966) exposed the animals orally to sodium tungstate at doses of $100,50,25$, and $10 \mathrm{mg} / \mathrm{kg}$ body weight (frequency of dosing was unspecified, but probably daily), presumably in drinking water for an unspecified period of time (probably not more than $30 \mathrm{~d}$ ). Blood cholinesterase (ChE) was monitored in the rats only; it was decreased at all doses tested but quantitative effects were not reported. These doses were sufficient to retard growth in all rat groups. 
Table F.4. Neurotoxicity of tungsten and its compounds in animals

\begin{tabular}{|c|c|c|c|c|c|}
\hline $\begin{array}{l}\text { Species/ } \\
\text { compound }\end{array}$ & Route & Dose & $\begin{array}{l}\text { Exposure } \\
\text { duration }\end{array}$ & Endpoints observed & References \\
\hline $\begin{array}{l}\text { Guinea } \\
\text { pig/sodium } \\
\text { tungstate }\end{array}$ & Gavage & $\begin{array}{l}0.78 \mathrm{~g} / \mathrm{kg} \text { body } \\
\text { wt ( } 2 \mathrm{Ss}) ; \\
1.3 \mathrm{~g} / \mathrm{kg}(1 \mathrm{~S})\end{array}$ & Acute (1 d) & $\begin{array}{l}\text { Loss of appetite, } \\
\text { trembling, } \\
\text { uncoordinated } \\
\text { movements, sudden } \\
\text { jumps, death }\end{array}$ & Karantassis 1924 \\
\hline $\begin{array}{l}\text { Guinea } \\
\text { pig/sodium } \\
\text { tungstate }\end{array}$ & Injection & $\begin{array}{l}0.75-0.84 \mathrm{~g} / \mathrm{kg} \\
\text { body wt }(3 \mathrm{Ss}) \\
2.5-2.7 \mathrm{~g} / \mathrm{kg} \\
\text { body wt }(2 \mathrm{Ss})\end{array}$ & Acute $(1 \mathrm{~d})$ & $\begin{array}{l}\text { Loss of appetite, } \\
\text { trembling, } \\
\text { uncoordinated } \\
\text { movements, sudden } \\
\text { jumps, death }\end{array}$ & Karantassis 1924 \\
\hline $\begin{array}{l}\text { Rat/sodium } \\
\text { tungstate }\end{array}$ & Oral & $\begin{array}{l}10,25,50 \\
100 \mathrm{mg} / \mathrm{kg}\end{array}$ & $\begin{array}{l}\text { Subacute (not } \\
\text { specified in d) }\end{array}$ & $\begin{array}{l}\text { Blood } \mathrm{ChE}^{a} \text { decreased } \\
\text { at all doses }\end{array}$ & Nadeenko 1966 \\
\hline $\begin{array}{l}\text { Rat/sodium } \\
\text { tungstate }\end{array}$ & Oral & $\begin{array}{l}0.005,0.05 \\
0.5 \mathrm{mg} / \mathrm{kg}\end{array}$ & $7-8$ months & $\begin{array}{l}\text { Blood ChE decreased at } \\
0.5 \mathrm{mg} / \mathrm{kg} \text { dose after } \\
4 \mathrm{month} \text {; decreased at } \\
0.05 \mathrm{mg} / \mathrm{kg} \text { in } \\
\text { 8th month }\end{array}$ & Nadeenko 1966 \\
\hline $\begin{array}{l}\text { Rat/sodium } \\
\text { tungstate }\end{array}$ & Oral & $\begin{array}{l}0.005,0.05 \\
0.5 \mathrm{mg} / \mathrm{kg}\end{array}$ & 7 months & $\begin{array}{l}\text { Conditioned learning } \\
\text { decreased at } 0.5 \mathrm{mg} / \mathrm{kg} \\
\text { and at } 0.05 \mathrm{mg} / \mathrm{kg} \\
\text { (dose-dependent } \\
\text { effects); no effects at } \\
0.005 \mathrm{mg} / \mathrm{kg}\end{array}$ & Nadeenko 1966 \\
\hline $\begin{array}{l}\text { Rat, rabbit } \\
\text { (?)/sodium } \\
\text { tungstate }\end{array}$ & Oral & $\begin{array}{l}0.005,0.05 \\
0.5 \mathrm{mg} / \mathrm{kg}\end{array}$ & 7-8 months & $\begin{array}{l}\text { Histopathologically } \\
\text { observed cerebral } \\
\text { cortex lesions at } \\
\text { sacrifice at } 5 \text {, } \\
0.5 \mathrm{mg} / \mathrm{kg} \text {, none at } \\
0.005 \mathrm{mg} / \mathrm{kg}\end{array}$ & Nadeenko 1966 \\
\hline $\begin{array}{l}\text { Rat/sodium } \\
\text { tungstate }\end{array}$ & Oral & $\begin{array}{l}0.005,0.05 \\
0.5 \mathrm{mg} / \mathrm{kg}\end{array}$ & 7 months & $\begin{array}{l}\text { Decreased } \mathrm{SH}^{a} \text { group } \\
\text { content of brain tissue } \\
\text { at } 0.5 \mathrm{mg} / \mathrm{kg}\end{array}$ & Nadeenko 1966 \\
\hline $\begin{array}{l}\text { Rat/sodium } \\
\text { tungstate } \\
\text { (assumed) }\end{array}$ & Oral & $0.005 \mathrm{mg} / \mathrm{kg}$ & 8 months & Reflex activity & $\begin{array}{l}\text { Nadeenko and } \\
\text { Lenchenko } 1977 \text {; } \\
\text { Rat/sodium } \\
\text { tungstate } \\
\text { Inhalation } 10,20 \text {, } \\
100,600 \mathrm{mg} / \mathrm{m}^{3} \\
30 \mathrm{~d} \text { Decreased } \\
\text { ChE at all doses, } \\
\text { behavior effects at } \\
100,600 \mathrm{mg} / \mathrm{m}^{3} \\
\text { Idiatullina } 1981\end{array}$ \\
\hline
\end{tabular}


Table F.4 (continued)

\begin{tabular}{|c|c|c|c|c|c|}
\hline $\begin{array}{l}\text { Rat/sodium } \\
\text { tungstate }\end{array}$ & Inhalation & $\begin{array}{l}0.1,0.5 \\
1.0 \mathrm{mg} / \mathrm{m}^{3}\end{array}$ & 4 months & $\begin{array}{l}\text { Decreased ChE at } 0.5 \text {, } \\
1.0 \mathrm{mg} / \mathrm{m}^{3} ; \\
\text { histopathologically } \\
\text { observed moderate } \\
\text { diffuse sclerosis in } \\
\text { brain at sacrifice }\end{array}$ & Idiatullina 1981 \\
\hline $\begin{array}{l}\text { Rat/sodium } \\
\text { tungstate }\end{array}$ & IP & $\begin{array}{l}0.5 \mu \mathrm{g} / \mathrm{d} ; \\
\text { estimated } 2 \times \\
10^{-6} \mathrm{mg} / \mathrm{kg} / \mathrm{d}\end{array}$ & $100 \mathrm{~d}$ & $\begin{array}{l}\text { Increased brain } \\
\text { glutaminase activity; no } \\
\text { effect on brain glutamic } \\
\text { acid levels }\end{array}$ & Capilna et al 1963 \\
\hline $\begin{array}{l}\text { Cat/tungstic } \\
\text { acid gel }\end{array}$ & $\begin{array}{l}\text { Direct } \\
\text { applicatio } \\
\mathrm{n} \text { to brain } \\
\text { surface }\end{array}$ & $0.02 \mathrm{~mL}$ & $\begin{array}{l}\text { One } \\
\text { application }\end{array}$ & $\begin{array}{l}\text { Focal EEG }{ }^{a} \text { seizure } \\
\text { activity }\end{array}$ & $\begin{array}{l}\text { Kusske, Wyler and } \\
\text { Ward } 1974\end{array}$ \\
\hline $\begin{array}{l}\text { Rat, cat, } \\
\text { monkey/ } \\
\text { tungstic } \\
\text { acid gel }\end{array}$ & $\begin{array}{l}\text { Direct } \\
\text { applicatio } \\
\mathrm{n} \text { to brain } \\
\text { surface }\end{array}$ & $0.01 \mathrm{~mL}$ & $\begin{array}{l}\text { One } \\
\text { application }\end{array}$ & $\begin{array}{l}\text { Focal EEG seizure } \\
\text { activity }\end{array}$ & $\begin{array}{l}\text { Black, Abraham } \\
\text { and Ward } 1967\end{array}$ \\
\hline
\end{tabular}

${ }^{a}$ Abbreviations: $\mathrm{ChE}=$ cholinesterase; $\mathrm{SH}=$ sulfhydryl groups; $\mathrm{EEG}=$ electroencephalogram.

Nadeenko (1966) then pursued chronic oral exposures of rats and rabbits to sodium tungstate and again reported results in rats indicative of neurotoxicity. Rats were given $0.5,0.05$, and $0.005 \mathrm{mg} / \mathrm{kg}$ body weight for 7 months (frequency of dosing unspecified, but assumed probably to be daily); while no control group is mentioned in the text, a figure showing effects on blood $\mathrm{ChE}$ includes data for a control group. Neither the rat strain nor number of rats per group is reported; in the rabbit experiments, 7 animals per group were exposed for 8 months to doses of $5,0.5,0.05$, and 0.005 $\mathrm{mg} / \mathrm{kg}$ at an unspecified frequency. The vehicle is not explicitly identified but the context suggests that all doses were given via drinking water.

Blood ChE levels were slightly depressed (to $80-85 \%$ of control level) at the higher dose levels after prolonged periods of exposure ( 4 months at $5 \mathrm{mg} / \mathrm{kg} ; 8$ months at $0.5 \mathrm{mg} / \mathrm{kg}$ ). Neither text nor figure specify whether ChE was monitored in rats or rabbits or both; the figure is ambiguous, showing data for an 8-month exposure period and high dose group of $5 \mathrm{mg} / \mathrm{kg}$, which were only supposed to have been present in the rabbit experiment. However, the figure legend indicates that results were obtained during reflex extinction, a procedure carried out according to the text only in the rat experiments.

Further and more convincing evidence of neurotoxic effects of low-dose tungstate exposure was accrued by Nadeenko (1966) in the course of studying CNS function by means of conditioned reflex learning in rats. Dose-dependent effects on several aspects of conditioning resulted and are presented graphically; no statistically significant effects were observed at the $0.005 \mathrm{mg} / \mathrm{kg}$ dose level.

Histopathological examination of tissue from animals treated with 5 and $0.5 \mathrm{mg} / \mathrm{kg}$ for $8 \mathrm{months}$ revealed morphological alterations in the cerebral cortex, while no effects were seen at $0.005 \mathrm{mg} / \mathrm{kg}$. No details regarding the lesion are presented, nor is it clear whether brain lesions were observed in both rabbits and rats, although the wording in the text is suggestive of both species showing effects. 
At sacrifice, the SH group content of rat brain and liver tissue was determined. Brain SH group content was significantly reduced only in the highest dose group $(0.5 \mathrm{mg} / \mathrm{kg})$; in liver, SH group content was lower in both the 0.5 and $0.05 \mathrm{mg} / \mathrm{kg}$ dose groups.

The tungsten content of several tissues from the chronically exposed animals was determined at the time of sacrifice, but, unfortunately, no quantitative presentation is made of the distribution data. Tungsten was also detected in the blood and organs of the control animals, but again, no quantitative data are presented. The $0.005 \mathrm{mg} / \mathrm{kg}$ dose resulted in an increased content only in the blood and intestine, while $0.05 \mathrm{mg} / \mathrm{kg}$ caused a statistically significant increase as well in the other organs and tissues evaluated (liver, femur, and kidneys). The greatest increases were seen in the highest dose groups, as expected. In the absence of quantitative data, it is not possible to link the effects indicative of neurotoxicity with the biokinetic model presented above.

A later study by Nadeenko and Lenchenko (1977) reports the results of chronically exposing rats perorally to low levels of tungsten and three other elements, molybdenum, copper, and arsenic separately and in various combinations. Many endpoints were observed including blood ChE activity and some type of undefined reflex activity. It appears that an 8-month treatment of rats with tungsten alone at $0.005 \mathrm{mg} / \mathrm{kg}$ had a significantly deleterious effect on reflex activity, changing one index of reflex activity by $35 \%$; molybdenum $(0.025 \mathrm{mg} / \mathrm{kg})$ alone changed it by $19 \%$, while tungsten and molybdenum together at these concentrations appear to have offset each other, changing the index by only 3\%. Blood $\mathrm{ChE}$ showed short-term changes at the beginning and the end of exposure in animals given a combination of tungsten and copper $(0.005 \mathrm{mg} / \mathrm{kg}$ each $)$. A statistically significant effect of tungsten alone on blood $\mathrm{ChE}$ was not observed. However, blood $\mathrm{ChE}$ was less variable and closer to control values in animals exposed to combinations of these elements than those exposed to the substances separately.

A later inhalation study on male rats (Idiatullina 1981) involving an acute/subacute phase and also subchronic (4 month or 17 week) exposures to sodium tungstate as fine powder also showed $\mathrm{ChE}$ depression both at high air levels (acute/subacute exposure to $10,20,100$, and $600 \mathrm{mg} / \mathrm{m}^{3}$ ) and at low levels $\left(0.5\right.$ and $1.0 \mathrm{mg} / \mathrm{m}^{3}$ for 4 months). In the subchronic study, blood ChE was depressed to $75 \%$ and $78 \%$ of control level at $1.0 \mathrm{mg} / \mathrm{m}^{3}$ and $0.5 \mathrm{mg} / \mathrm{m}^{3}$, respectively. Only at $0.1 \mathrm{mg} / \mathrm{m}^{3}$ was there no effect seen. No data are reported on the extent of depression in the acute experiment but rather on the duration of exposure needed to produce a statistically significant depression; these values ranged from $18 \mathrm{~h}$ for $600 \mathrm{mg} / \mathrm{m}^{3}$ to $720 \mathrm{~h}$ for $10 \mathrm{mg} / \mathrm{m}^{3}$. Behavioral effects (muzzle scratching and increased activity, interpreted by the author as anxiety manifestations) appeared at 100 and $600 \mathrm{mg} / \mathrm{m}^{3}$. Histological examination of tissue from chronically-exposed rats ( $2 / 15$ per group) revealed moderate diffuse sclerosis in the brains (as well as heart and lungs) of the animals exposed to $1.0 \mathrm{mg} / \mathrm{m}^{3}$, with less evident changes at $0.5 \mathrm{mg} / \mathrm{m}^{3}$ and no changes observed at $0.1 \mathrm{mg} / \mathrm{m}^{3}$.

Another line of investigation suggestive of possible neurotoxic effects was pursued by Capilna et al (1963) who exposed rats to daily $0.5 \mu \mathrm{g}$ intraperitoneal (IP) injections of ammonium molybdate or sodium tungstate for 100 days. The strain, age, weight, and gender of the rats are not specified. Thus a dose in $\mathrm{mg} / \mathrm{kg}$ cannot be calculated for comparison with other studies such as those of Nadeenko (1966); if the animals were adults weighing around $250 \mathrm{~g}$, the dose would be estimated at $2 \times 10^{-6}$ $\mathrm{mg} / \mathrm{kg} / \mathrm{d}$, an extremely low dose. Even with 100 days of exposure, the total dose would have been only $0.0002 \mathrm{mg} / \mathrm{kg}$. Brain and liver glutaminase activity and brain glutamic acid levels were assayed in unexposed control animals and treated animals at 40,60, 80, and 100 days "following poisoning" (it is not clear whether this means after the onset of treatment or after the cessation of 100 days of 
exposure). Effects on enzyme activity levels showed progressively increasing effects with increasing duration of time, suggesting that the treatment groups were sacrificed at times following the onset of treatment.

Tungsten treatment activated brain glutaminase activity in contrast to molybdenum which inhibited it (Capilna et al 1963). Brain glutamic acid levels remained unchanged by tungstate, suggesting a concurrent activation of glutamic acid oxidation. Brain glutamic acid concentration was significantly depressed by molybdate exposure in conjunction with the inhibition of glutaminase activity. Liver glutaminase activity was decreased both by tungstate and molybdate treatment; this may have been effected by the metals binding either SH groups or carboxylic acid groups on the enzyme molecules. The elevation of brain glutaminase activity would be expected to result in increased ammonia levels although these were not measured in this study. No functional or behavioral observations were reported to indicate whether there were observable correlates of the metal exposures.

A further possible indicator of neurotoxic effects of tungsten in at least one form is the use of direct application of pure tungstic acid gel to the cortex of experimental animals to induce focal epileptic or seizure activity. This use is reviewed by Kusske, Wyler, and Ward (1974), while details of the methods are presented by Black, Abraham and Ward (1967). The latter group points out that only one of three forms of tungstic acid is biologically active, the colloidal form. The monohydrate is insoluble in water and biologically inert; the hydrous form is slightly soluble in water but also biologically inert. Phosphotungstic acid, a protein precipitating agent, is also not effective in inducing seizure activity, according to these authors. The seizure activity induced by $0.01 \mathrm{~mL}$ tungstic acid gel is usually temporary and is accompanied by histologically verifiable spherical necrotic lesions of about $2.8 \mathrm{~mm}$ diameter (Black, Abraham and Ward 1967). Whether this effect of tungsten has any relevance to environmental exposures is open to question in view of the specificity of the form and method of application required to produce the epileptogenic effect.

\section{F.2.1.3 Relationship to Biokinetic Model}

Distribution data from rodent studies (e.g., Kaye 1968) permit the estimation of rat brain tungsten levels from the doses and exposure times employed by Nadeenko (1966) and Nadeenko and Lenchenko (1977). Assuming an oral intake of $1 \mathrm{mg} \mathrm{W} / \mathrm{rat} / \mathrm{d}$, the brain tissue levels are estimated to be $0.00005 \mathrm{mg} \mathrm{W} / \mathrm{g}$ tissue after $30 \mathrm{~d}, 0.00010 \mathrm{mg} \mathrm{W} / \mathrm{g}$ after $4 \mathrm{mo}, 0.00014 \mathrm{mg} \mathrm{W} / \mathrm{g}$ after $7 \mathrm{mo}$, and $0.00014 \mathrm{mg} \mathrm{W} / \mathrm{g}$ after $8 \mathrm{mo}$. The uncertainty in each estimate is at least a factor of two, and probably greater for the exposure durations of more than 30. Table F.5 shows the corresponding estimates of rat brain tissue content for the dose levels and exposure times employed by Nadeenko (1966).

Estimation of tissue levels from the inhalation study of Idiatullina (1981) is more difficult as only a study in dogs is available as a basis for estimating the rate of absorption of tungsten from lungs to blood (Aamodt 1975), but a unit intake of tungsten is estimated to result in about half the brain level achieved by oral exposure (inhalation of $1 \mathrm{mg} \mathrm{W} / \mathrm{d} / \mathrm{rat}$ might yield $0.00002-0.00003 \mathrm{mg}$ W/g brain tissue at $30 \mathrm{~d}$ exposure). The uncertainty in this estimate is probably more than a factor of two.

Estimates are shown in Table F.6 of the tissue levels of tungsten in the human soft tissue remainder (those soft tissues not explicitly modeled in the biokinetic model developed in Appendix C) for several durations of ingestion exposure. The brain and gonads are included in the remainder tissues. The level of uncertainty is considered to be high, at least a factor of ten for this average tissue level estimate. It would be even higher for individual tissues, particularly the brain, as the blood-brain barrier may affect 
Table F.5. Levels of tungsten in rat tissues resulting from unit ingestion exposure

\begin{tabular}{cccc}
\hline $\begin{array}{c}\text { Exposure duration, } \\
\text { month }\end{array}$ & Units W/g brain & $\begin{array}{c}\text { Mg W/g brain tissue } \\
\text { at } 0.5 \mathrm{mg} / \mathrm{g} / \mathrm{d}\end{array}$ & $\begin{array}{c}\mathrm{Mg} \mathrm{W} / \mathrm{g} \text { brain tissue } \\
\text { at } 0.005 \mathrm{mg} / \mathrm{kg} / \mathrm{d}\end{array}$ \\
\hline 1 & 0.00005 & 0.000025 & 0.00000025 \\
4 & 0.00010 & 0.00005 & 0.0000005 \\
7 & 0.00014 & 0.00007 & 0.0000007 \\
8 & 0.00014 & 0.00007 & 0.0000007 \\
\hline
\end{tabular}

${ }^{\sigma}$ Estimation of $\mathrm{W}$ accumulation supplied by $\mathrm{R}$. W. Leggett, based on distribution data from rat studies. Uncertainty at least a factor of two.

Table F.6. Levels of tungsten in human soft tissues resulting from unit ingestion exposure

\begin{tabular}{ccc}
\hline $\begin{array}{c}\text { Exposure duration, } \\
\text { month }\end{array}$ & $\begin{array}{c}\text { Units tungsten in total } \\
\text { remainder tissue }\end{array}$ & Units tungsten $/ \mathrm{g}^{b}$ \\
\hline 1 & 0.475 & 0.00000475 \\
4 & 0.75 & 0.0000075 \\
7 & 0.93 & 0.0000093 \\
8 & 0.99 & 0.0000099 \\
\hline
\end{tabular}

${ }^{a}$ Calculation supplied by R. W. Leggett, based on biokinetic model presented in Appendix C.

${ }^{b}$ Assumes mass of "remainder" soft tissue $=50 \mathrm{~kg}$ for $70 \mathrm{~kg}$ person; GI uptake factor $=0.5$ as in biokinetic model (see Appendix C). Uncertainty at least a factor of 10 , probably more for brain tissue due to possible effects on uptake by blood-brain barrier.

the uptake by the brain considerably. No autopsy data are available for humans against which to validate these estimates; they would be of great value.

\section{F.2.1.4 Evaluation of Significance and Identification of Data Gaps}

Two studies of workers exposed to hard metal dust report signs and symptoms suggestive of CNS effects (Kaplun and Mezentseva 1959; Vengerskaya and Salikhodzhaev 1962). In both studies, workers were found who complained of loss of appetite, loss of the sense of smell, and nausea. In the second study, dizziness and headache were also reported. Unfortunately, interpretation of these studies is difficult because the hard metal dusts contained not only tungsten but also cobalt and possibly small amounts of other metals.

A small recent study of hard metal workers who complained of memory problems and also had pulmonary complaints confirmed specific verbal memory and attention deficits upon testing (Jordan et al 1990). Like those above, this study is also confounded by the presence of cobalt and possibly other metals in the dusts to which the workers were exposed. An important additional confounder is the use of an organic solvent (unspecified) in the mists to which they were also exposed. Many organic solvents are known or suspected neurotoxicants. Thus the role of tungsten cannot be clearly defined and further study would be needed to elucidate the causal agent(s) for these effects. For those 
workers with compromised pulmonary function, chronically low brain oxygen levels cannot be excluded as causing or contributing to impaired brain function. No measures of arterial $\mathrm{pO}_{2}$ were presented in any of these human studies.

Animal studies, particularly those of Nadeenko (1966), Nadeenko and Lenchenko (1977), and Idiatullina (1981) taken together, are somewhat more convincing in terms of suggesting that tungsten, particularly as sodium tungstate, may have a neurotoxic effect, even at low doses in long-term exposure by an environmentally relevant route (oral dosing). Nadeenko (1966) observed a decrease in blood ChE activity at all doses $(10,25,50$, and $100 \mathrm{mg} / \mathrm{kg}$, presumably per day) in rats in a subacute study of unspecified duration. No quantitative $\mathrm{ChE}$ results of the subchronic study were presented. He also reported a slight $\mathrm{ChE}$ decrease (to $80-85 \%$ of control level) at the higher doses of sodium tungstate $(0.05,0.5 \mathrm{mg} / \mathrm{kg}$ ) administered in a chronic study ( $7-8$ months). The rats given the doses resulting in lowered blood $\mathrm{ChE}$ also showed dose-dependent decrements in several aspects of conditioned learning. The lowest dose, $0.005 \mathrm{mg} / \mathrm{kg}$, had no effect on blood $\mathrm{ChE}$ or on learning over the period of study. Furthermore, the higher two dose groups showed histologically-observed cerebral cortex lesions at necropsy as well as lower brain tissue content of SH groups at the $0.5 \mathrm{mg} / \mathrm{kg}$ dose level. While the text is not clear, it appears that chronically-exposed rabbits in the higher dose groups also may have had cerebral cortex lesions at sacrifice.

In the later Nadeenko and Lenchenko (1977) study, $0.005 \mathrm{mg} / \mathrm{kg}$ tungsten perorally for 8 months had an effect on reflex activity (type of reflexes not specified) and a possible, though not statistically significant, effect on blood ChE activity.

The effects of oral tungstate exposure on $\mathrm{ChE}$ depression and brain microstructure are corroborated by the effects produced by inhalation exposure of rats to high (acute/subacute study) and low levels (subchronic study) (Idiatullina 1981). Depression of blood ChE to 75-78\% of control levels was observed in the 4-month study. Diffuse sclerosis was seen on histopathological evaluation of the brains of these animals. Effects on behavior were observed at the highest tungsten air levels; these were interpreted by the author as indicating anxiety; to this reviewer, they suggest an aversive response to possible irritation of nasal passages.

If long-term, low-dose exposure to tungsten compounds does indeed affect the cholinergic pathways of the brain as suggested by the Nadeenko and Idiatullina studies, effects on mood and mental function might be expected as shown by studies of workers exposed to anticholinesterase insecticides and also workers and volunteers exposed to organophosphate (OP) nerve agents. For example, Savage et al (1988) evaluated 100 workers who had at least one documented exposure to OP insecticides resulting in signs and symptoms of acute systemic toxicity. These episodes had occurred on average nine years prior to evaluation by Savage and colleagues. The group evidenced mild but statistically significant deficits in various measures of mental function on standardized tests, showed adverse effects on mood and behavior (increased irritability, confusion, and tendency to withdrawal) as evaluated by relatives, and themselves perceived difficulty with memory, thinking ability, and use of language.

Organophosphate insecticides tend to be stored in body fat and released slowly over long periods of time, thus mimicking somewhat a long-term, low dose environmental exposure.

Metcalf and Holmes (1969) evaluated workers exposed to OPs including insecticides and nerve agents. They found memory, concentration, and sleep disturbances as well as minor motor coordination deficits and subtle EEG changes in the "highly exposed" as compared to the "minimally exposed" worker groups. 
Levin, Rodnitzky, and Mick (1976) compared two subgroups of workers (24 Ss total) having potential organophosphate insecticide exposure but no signs or symptoms of systemic toxicity with a control group for indications of anxiety and depression. They found that the group of commercial insecticide applicators showed elevated levels of anxiety (tension, restlessness, anxiety, and sleep disturbance) and plasma ChE at $70 \%$ of the control group level. Exposed farmers neither had depressed plasma ChE nor elevated scores for anxiety or depression. RBC-ChE was not depressed in either group.

Acute experimental exposure of volunteers to agent VX, a potent OP nerve agent, in doses sufficient to lower blood and RBC-ChE, has been shown to have transient effects on mental function and mood as documented by Bowers, Goodman, and Sim (1964) and Sidell and Groff (1974). The former group tested VX [identified only by them as EA-1701, but this code is equivalent to VX (Krackow 1956)] in 93 volunteers exposed percutaneously to the agent. In both studies, effects on mental function were evident with blood $\mathrm{ChE}$ depressed to 10 to $40 \%$ of control or baseline activity levels. Two subjects in the Bowers, Goodman and Sim (1964) study evidenced CNS effects with ChE depression only at 81 to $100 \%$ of baseline, and a few more at the intermediate 41 to $80 \%$ level.

Acute accidental exposure to the OP nerve agent GB has also resulted in both transient and prolonged psychological changes [as reviewed by Munro, Ambrose, and Watson (1994)]. These include depression, insomnia, and excessive dreaming. Occupationally exposed workers have shown similar signs and symptoms after low-level G-agent exposures as well as nervousness, drowsiness, mood changes, weakness and easy fatigability, with effects persisting more than 3 days in some cases (Brown 1948; Craig and Cornblath 1953).

From personal observation, Sidell (1992) considers mild psychological disturbances to occur not uncommonly in persons with few or no physical signs of nerve agent exposure. He describes the effects as beginning hours to a day after exposure and persisting variably from days to many weeks. The changes characteristically include forgetfulness and slowed thought processes, and may also include irritability or impairment of judgement, comprehension, or communication ability.

Overall, these reports suggest that changes in mood and mental function associated with acute anticholinesterase exposure [and presumably concomitant excess acetylcholine (ACh) accumulation in the CNS] are more likely to be observed after acute exposure sufficient to lower blood ChE to the $10-40 \%$ of baseline range, but also are seen in a few individuals with depression only to the 81-100\% of baseline range, or that range corresponding to the depression induced by tungsten exposure in animal studies.

Thus, if chronic tungsten exposure does have an anticholinesterase effect, causing an accumulation of ACh in the CNS, effects of the type observed by Jordan et al (1990) on attention and memory would be consistent with the responses seen in humans exposed to OP anticholinesterase compounds.

The issue as to whether long-term, low-dose exposure of humans to tungsten causes any type of neurotoxicity is of considerable importance to resolve. It is of major significance in view of the potential consequences for interpersonal relationships, academic potential, and employment from even fairly subtle effects on mood, memory, attention, and cognitive functions.

Neurotoxicity is considered to be potentially of much more significance than the possibility for minor nephrotoxic effects of uranium on kidney function from long-term, very low dose exposure in view of the very large functional reserve ( $>1$ kidney) in persons of normal kidney function. Neurotoxicity has 
the potential to affect all exposed individuals, while nephrotoxic effects would be of significance only to the small minority of individuals lacking a kidney or with significantly compromised kidney function.

Furthermore, neurotoxicity including effects on learning, memory, and attention in adult individuals raises the question of possible developmental neurotoxicity if individuals are exposed in utero and/or during postnatal stages of brain development. Children with compromised ability to attend appropriately and having short-term memory deficits commonly associated with Attention Deficit Disorder are widely recognized to have significant difficulties in their school, home, and social environments. These difficulties typically persist and have adverse consequences for later academic achievement and performance in the workplace as well as interpersonal relationships. No studies of developmental neurotoxicity in animals or humans have been located. Recommendations are discussed in the section below on developmental toxicity.

\section{F.2.1.5 Tentative Dose-Response Relationship}

Studies on rats by Nadeenko (1966) revealed a dose-dependent effect of long-term, low-dose sodium tungstate exposure on several aspects of conditioned learning, with a possible threshold, as no effect was seen at the $0.005 \mathrm{mg} / \mathrm{kg}$ body weight dose level. Blood ChE activity was lowered, not with a dose-response effect in terms of degree of inhibition, but with respect to the time required to reach the maximum effect. Again, an apparent threshold was demonstrated, as no effect was observed at the $0.005 \mathrm{mg} / \mathrm{kg}$ dose, at least during the 7 or $8 \mathrm{month}$ period of exposure and observation. Furthermore, no brain lesions were seen in the lowest dose group at sacrifice.

However, in a later study, Nadeenko and Lenchenko (1977) reported significantly adverse effects on certain undefined indices of reflex activity in rats exposed orally to $0.005 \mathrm{mg} / \mathrm{kg}$ tungsten for 8 months. Thus, if there is a true threshold for neurotoxic effects, it may be at a yet lower dose. Idiatullina (1981) demonstrated a dose-dependent relationship between duration of inhalation exposure to sodium tungstate and the attainment of a statistically significant depression of blood $\mathrm{ChE}$ in an acute/subacute study; the degree of $\mathrm{ChE}$ lowering was not reported. In a subchronic portion of the study, Idiatullina did not observe a dose response effect on ChE activity, but rather a step function type of response. In this phase of the study, $0.1 \mathrm{mg} / \mathrm{m}^{3}$ for 4 months gave no effect, while 0.5 and $1.0 \mathrm{mg} / \mathrm{m}^{3}$ both resulted in about the same degree of activity inhibition ( 78 and $75 \%$ of control activity, respectively).

\section{F.2.1.6 Research Recommendations}

It would be of considerable interest to determine whether long-term exposure of workers to tungsten or tungsten carbide compounds without concomitant cobalt or organic solvent exposure results in lowering of blood or RBC-ChE. Tests of memory and mental function together with assessment of mood changes would also be most useful. Such human populations may not be available. Prior to initiating human studies, or as an alternative, it would seem prudent to determine whether the animal results on blood $\mathrm{ChE}$ activity, learning, behavior, and brain histopathology can be replicated and to determine the corresponding tungsten tissue distribution quantitatively.

An initial subacute study in rats is recommended at sodium tungstate dose levels corresponding to those used in the Nadeenko (1966) subacute rat study, or $100,50,25$, and $10 \mathrm{mg} / \mathrm{kg}$ in drinking water. Effects on blood and brain $\mathrm{ChE}$, behavior, learning, and tissue $\mathrm{W}$ distribution should be monitored. If 
effects on ChE activity and/or the other endpoints are observed, then a low-dose chronic study similar to that reported by Nadeenko (1966) would be warranted. Dose levels of $1,0.5,0.05$, and $0.005 \mathrm{mg} / \mathrm{kg}$ would be desirable. Tissue and blood levels of tungsten would be essential to obtain at the time of sacrifice.

If significant effects are observed in a rat study, especially on learning endpoints, consideration of studying neurotoxicity endpoints in a larger, nonrodent, species would be warranted.

\section{F.2.2 Reproductive Toxicity}

\section{F.2.2.1 Description of Effects}

Nadeenko et al (1977) reported pre- and postimplantation embryotoxic effects on rats resulting from chronic $(8 \mathrm{mo}$ ) oral tungsten exposure at $0.005 \mathrm{mg} / \mathrm{kg}$ as well as molybdenum at $0.025 \mathrm{mg} / \mathrm{kg}$ (see Table F.7). The form of tungsten is not specified, but is assume to have been sodium tungstate as in Nadeendo (1966); it is further assumed that the compound was given in drinking water. Nadeenko and Lenchenko (1977) exposed rats orally for 8 months to tungsten, molybdenum, arsenic, and copper in various combinations at three dose levels and separately as follows: tungsten $(0.005 \mathrm{mg} / \mathrm{kg})$, molybdenum $(0.025 \mathrm{mg} / \mathrm{kg})$, arsenic $(0.0025 \mathrm{mg} / \mathrm{kg})$, and copper $(0.005 \mathrm{mg} / \mathrm{kg})$. They found that tungsten significantly increased the preimplantation embryonic death rate while arsenic significantly increased postimplantation embryolethality. A combination of all four metals at the same concentrations listed above increased preimplantation embryolethality to an essentially identical extent as tungsten alone. This information has important implications for future investigations of tungsten in conjunction with possible alloying elements. These dates suggest that not only is there no synergism, but there is also no evidence of additive effects.

Nadeenko et al (1978) presented in a more detailed way some of the same data as in Nadeenko and Lenchenko (1977) on increased preimplantation embryonic lethality after administering $0.005 \mathrm{mg} / \mathrm{kg}$ tungsten perorally to adult female rats for 7 months including through pregnancy. The authors speculated that the effect was attributable to direct embryotoxic effects, as tungsten was found at increased concentration in the fetuses (but not the placentas) at doses causing the observed effects.

In contrast, Chiang et al (1981 Abstract) found no embryotoxic effects in products of conception of female Wistar rats made sulfite oxidase-deficient by exposure to a high tungsten/low molybdenum regimen for 4 weeks followed by sulfite in drinking water for 3 weeks prior to mating and throughout the 3 weeks of pregnancy. No effects were seen on litter size, number of resorptions, or mean pup weight. The differences from Nadeenko's observations may be attributable to strain differences, the substantial difference in duration of tungsten exposure, or other differences in experimental design.

Wide (1984) injected some groups of mice on the 3rd and others on the 8th day of pregnancy with sodium tungstate, $0.1 \mathrm{~mL}$ of $25 \mathrm{mM}$ in saline via tail vein (equivalent to $18.4 \mathrm{mg} / \mathrm{kg}$ as W), and found that injection on day 8 significantly increased the number of fetal resorptions. No effects were seen on skeletal ossification or any other aspects of fetal development as evaluated at sacrifice on day 17 of pregnancy. Tungsten on day 3 did not interfere with implantation or subsequent development. The author noted that it was not possible to determine whether the increased fetal lethality was a result of direct fetal effects or whether tungsten disrupted the maternal environment responsible for supporting the pregnancy. 
Table F.7. Reproductive toxicity of tungsten

\begin{tabular}{|c|c|c|c|c|c|c|}
\hline $\begin{array}{l}\text { Species/ } \\
\text { compound }\end{array}$ & Route & Dose & $\begin{array}{l}\text { Exposure } \\
\text { duration }\end{array}$ & Tissue level & $\begin{array}{l}\text { Endpoints } \\
\text { observed }\end{array}$ & References \\
\hline $\begin{array}{l}\text { Mouse/ } \\
\text { sodium } \\
\text { tungstate }\end{array}$ & $\mathrm{IV}^{a}$ & $\begin{array}{l}0.1 \mathrm{~mL} \text { of } \\
25 \mathrm{mM} \text { sol'n } \\
(18.4 \mathrm{mg} \mathrm{W} / \mathrm{kg})\end{array}$ & $\begin{array}{l}1 \text { injec @ d 3, } \\
1 \text { injec @ d } 8\end{array}$ & $\mathrm{NA}^{a}$ & $\begin{array}{l}\text { Increased fetal } \\
\text { resorption }\end{array}$ & Wide 1984 \\
\hline $\begin{array}{l}\text { Mouse/ } \\
\text { sodium } \\
\text { tungstate }\end{array}$ & IV & $\begin{array}{l}\text { A. } 120 \mu \mathrm{g} \\
{ }^{185} \mathrm{~W} / \mathrm{kg} \\
\text { B. } 120 \mu \mathrm{g} \\
{ }^{185} \mathrm{~W} / \mathrm{kg}+20 \mathrm{mg} \\
\text { cold } W / \mathrm{kg}\end{array}$ & $\begin{array}{l}1 \text { injec @ d 12, } \\
1 \text { injec @ d 17 }\end{array}$ & $\begin{array}{l}\text { Det'n in dam, } \\
\text { fetuses; } 1 \mathrm{~h}, \\
4 \mathrm{~h} \text { post- } \\
\text { injection }\end{array}$ & $\begin{array}{l}\text { Transfer to } \\
\text { placenta and } \\
\text { fetus }\end{array}$ & Wide et al 1986 \\
\hline $\begin{array}{l}\text { Rat/sodium } \\
\text { tungstate }\end{array}$ & Oral & $0.005 \mathrm{mg} / \mathrm{kg}$ & $\begin{array}{l}\text { Chronic, } \\
8 \text { months }\end{array}$ & $\begin{array}{l}1.97 \pm \\
0.2 \mu \mathrm{g} \% \text { in } \\
\text { fetus }\end{array}$ & $\begin{array}{l}\text { Embryotoxicity; } \\
\text { transfer to fetus }\end{array}$ & $\begin{array}{l}\text { Nadeenko and } \\
\text { Lenchenko } 1977\end{array}$ \\
\hline $\begin{array}{l}\text { Rat/sodium } \\
\text { tungstate }\end{array}$ & Oral & $0.005 \mathrm{mg} / \mathrm{kg}$ & $\begin{array}{l}\text { Chronic, } \\
7 \text { months }\end{array}$ & NA & $\begin{array}{l}\text { Preimplantation } \\
\text { embryotoxicity }\end{array}$ & Nadeenko et al 1978 \\
\hline $\begin{array}{l}\text { Rat/sodium } \\
\text { tungstate }\end{array}$ & Inhalation & $\begin{array}{l}1.0 \mathrm{mg} / \mathrm{m}^{3} \\
0.5 \mathrm{mg} / \mathrm{m}^{3}\end{array}$ & $\begin{array}{l}\text { Subchronic; } \\
4 \text { months }\end{array}$ & NA & $\begin{array}{l}\text { Decreased } \\
\text { sperm motility }\end{array}$ & Idiatullina 1981 \\
\hline $\begin{array}{l}\text { Mousel } \\
\text { sodium } \\
\text { tungstate }\end{array}$ & Oral & NA & Chronic & NA & $\begin{array}{l}\text { Decreased blood } \\
\text { progesterone in } \\
\text { pregnant female }\end{array}$ & Wide et al 1986 \\
\hline $\begin{array}{l}\text { Laying } \\
\text { hen/ } \\
\text { tungsten }\end{array}$ & Oral & $\begin{array}{l}1 \mathrm{~g} \text { tungsten } / \mathrm{kg} \\
\text { feed }\end{array}$ & $\begin{array}{l}\text { Subchronic; } \\
5 \text { months }\end{array}$ & NA & $\begin{array}{l}\text { Decreased egg } \\
\text { production; } \\
\text { decreased egg } \\
\text { (E) weight, yolk } \\
\text { (Y) weight, Y:E } \\
\text { weight ratio }\end{array}$ & $\begin{array}{l}\text { Nell et al } 1981 \text { Chick } \\
\text { embryo limb bud cell } \\
\text { culture/sodium } \\
\text { tungstate In vitro } 0 \text {, } \\
75,150,200 \text {, } \\
250 \mu \mathrm{M} 5 \text { d Same as } \\
\text { medium Decreased } \\
\text { cartiledge formation in } \\
\text { limb bud cells Wide } \\
\text { et al } 1986\end{array}$ \\
\hline $\begin{array}{l}\text { Rat ovary/ } \\
\text { sodium } \\
\text { tungstate }\end{array}$ & In vitro & $0-10 \mathrm{mM}$ & $20 \mathrm{~min}$ & NA & $\begin{array}{l}\text { Biphasic effect } \\
\text { on adenyl } \\
\text { cyclase function }\end{array}$ & Hwang and Ryan 1981 \\
\hline $\begin{array}{l}\text { Hen } \\
\text { oviduct } \\
\text { progesteron } \\
\text { e receptors } \\
\text { sodium } \\
\text { tungstate }\end{array}$ & In vitro & $0-20 \mathrm{mM}$ & NA & NA & $\begin{array}{l}\text { Blocking of } \\
\text { steroid binding } \\
\text { by receptor }\end{array}$ & $\begin{array}{l}\text { Murakami, } \\
\text { Quattrociocchi et al } \\
1982\end{array}$ \\
\hline $\begin{array}{l}\text { Chick } \\
\text { oviduct } \\
\text { progesteron } \\
\text { e receptors/ } \\
\text { sodium } \\
\text { tungstate }\end{array}$ & In vitro & $0-10 \mathrm{mM}$ & $\mathrm{NA}$ & NA & $\begin{array}{l}\text { Blocking of } \\
\text { receptor } \\
\text { activation; } \\
\text { blocking of } \\
\text { activated } \\
\text { receptor binding } \\
\text { to DNA }\end{array}$ & $\begin{array}{l}\text { Murakami, Szocik } \\
\text { et al } 1982\end{array}$ \\
\hline
\end{tabular}

${ }^{a}$ Abbreviations: IV = intravenous; $\mathrm{NA}=$ not available. 
In a later study, Wide, Danielsson, and Denker (1986) also observed fetal uptake of ${ }^{185} \mathrm{~W}$ after acute injection of pregnant mice, substantiating the evidence from Nadeenko's laboratory that tungsten crosses the placenta. Fetal uptake was low on Day 8 of gestation, increasing substantially by Day 12 . Autoradiographic data showed selective uptake by fetal nervous system tissue $4 \mathrm{~h}$ after injection on Day 12 of gestation.

Wide, Danielsson and Dencker (1986) also observed that tungstate inhibited cartilage formation in chick limb bud mesenchymal cultures in a dose-related manner. The inhibition was observed at concentrations (150-200 $\mu \mathrm{M})$ generally equivalent to those in vivo at $1 \mathrm{~h}$ after dosing with IV sodium tungstate, $20 \mathrm{mg} / \mathrm{kg}$, on day 12 of gestation $(100 \mu \mathrm{M})$. These lines of evidence suggest that tungsten could indeed have direct fetotoxic effects. However, the maternal pituitary and ovaries as well as other organs both accumulated and retained tungsten; thus, effects on the maternal pituitary-ovarian-placental endocrine functions necessary for support of pregnancy are also a possibility.

This is especially plausible in view of earlier work showing biphasic effects of tungstate on a key enzyme, adenyl cyclase, that is involved in mediating pituitary gonadotrophic hormone control of ovarian function (Hwang and Ryan 1981). Sodium tungstate was found to stimulate rat ovarian adenyl cyclase in vitro maximally at concentrations between 0.5 and $1 \mathrm{mM}$; higher concentrations were inhibitory. It also activated adenyl cyclase in several other rat tissues. Wide's group cited unpublished observations from their laboratory that may relate to this potential for tungsten's disruption of ovarian function; they observed a significant decrease in circulating progesterone levels in pregnant mice that had been exposed chronically to tungsten via drinking water (Wide, Danielsson and Dencker 1986). Furthermore, radiotungsten was observed by autoradiography to accumulate in ovarian follicles but not corpora lutea of pregnant mice injected on Day 8 of gestation with ${ }^{185} \mathrm{~W}$ (Wide, Danielsson, and Denker 1986). It was also seen to accumulate in placental structures and the pituitary gland.

It is not known to what extent avian and rat ovarian function are comparable hormonally, but Nell et al (1981) found that chronic oral exposure of laying hens to tungsten in the diet reduced egg production by $50 \%$. Hatchability of those eggs produced was not affected, although mean egg weight, fresh yolk weight, and the ratio of yolk to egg weight were significantly lessened.

Others have found that sodium tungstate blocks the activation of progesterone receptors and also the binding of activated progesterone receptors to DNA in hen and chick oviduct preparations (Murakami, Quattrociocchi, Szocik, et al 1982; Murakami, Szocik et al 1982). Thus, tungsten also appears to have the potential to interfere with the action of progesterone on its target tissue where it is needed to establish conditions necessary for maintenance of pregnancy.

Tungsten may have deleterious effects as well on male reproductive potential. Idiatullina (1981) exposed rats subchronically (17 weeks) via inhalation to sodium tungstate powder and observed a modest decrease in sperm motility at exposure levels of 1.0 and $0.5 \mathrm{mg} / \mathrm{m}^{3}$ but not at $0.1 \mathrm{mg} / \mathrm{m}^{3}$. Five out of 15 rats per group were evaluated. No dose response relationship was observed; the motility was $88 \%$ and $90 \%$ of control at 1.0 and $0.5 \mathrm{mg} / \mathrm{m}^{3}$ respectively. No effects were observed on the "acidic and osmotic resistance" of the spermatozoa.

Tungsten also has the potential to cause testicular atrophy and loss of spermatogenesis indirectly by inhibiting sulfite oxidase activity. This was shown in 21-week exposures of Sprague-Dawley rats to sodium tungstate in drinking water concomitant with feeding a molybdenum-deficient diet (Gunnison et al 1987). The testicular damage was attributed to endogenous sulfite; it was seen in $17 \%$ of animals 
given $400 \mathrm{ppm}$ and $90 \%$ of animals given $800 \mathrm{ppm}$ tungstate. Neither testes weight nor degenerative changes showed any reversal during a 4-week recovery period. According to Gunnison et al (1987), 98-99\% of sulfite oxidase activity must be absent before endogenous sulfite levels increase to detectable levels in rat blood. This is achievable only with extremely abnormal levels of Mo and $\mathrm{W}$ in the diet and thus is not relevant to the exposure scenarios of interest in this evaluation.

\section{F.2.2.2 Relation to Biokinetic Model}

As noted above, the distribution study of Wide, Danielsson, and Denker (1986) included a high-dose group given, in addition to $120 \mu \mathrm{g}{ }^{185} \mathrm{~W} / \mathrm{kg}$, a nearly identical IV dose $(20 \mathrm{mg} \mathrm{W} / \mathrm{kg})$ of unlabelled sodium tungstate as that $(18.4 \mathrm{mg} \mathrm{W} / \mathrm{kg}$ ) shown by Wide (1984) to cause increased fetal resorptions when administered on Day 8 of gestation. The high-dose group was exposed, however, on Day 12 of gestation, so the results may not be entirely equivalent (the fetal concentration would be expected to be higher on Day 12 than on Day 8 if the increasing trend seen from Day 12 to Day 17 can be extrapolated backwards). At one hour after administration on Day 12 , the concentration of ${ }^{185} \mathrm{~W}$ in the fetus was essentially the same $(104 \%)$ as in the low-dose experiment, $107 \pm 5.93 \mathrm{ng} / \mathrm{g}$ tissue. Four hours after injection, the fetal ${ }^{185} \mathrm{~W}$ concentration had fallen to $88 \%$ of $41.4 \mathrm{ng} / \mathrm{g}$ tissue or $36.4 \mathrm{ng} / \mathrm{g}$.

Placental tungsten concentration in mice $1 \mathrm{~h}$ after Day 12 injection of $120 \mu \mathrm{g}{ }^{185} \mathrm{~W} / \mathrm{kg}$ as sodium tungstate was $130 \pm 5.43 \mathrm{ng} \mathrm{W/g}$ tissue; this fell to $35.2 \pm 3.72 \mathrm{ng} \mathrm{W/g}$ at 4-h post-injection and to $0.86 \pm 0.09 \mathrm{ng}$ W/g at $48 \mathrm{~h}$ post injection (Wide, Danielsson, and Denker 1986). In the high-dose group (given $20 \mathrm{mg} \mathrm{W} / \mathrm{kg}$ unlabelled W in addition to $120 \mu \mathrm{g}{ }^{185} \mathrm{~W} / \mathrm{kg}$ ), the placental concentration at $1 \mathrm{~h}$ post injection was essentially the same as the low dose group (97\%); at 4 h post-injection, it was $69 \%$ or $24.3 \mathrm{ng} \mathrm{W/g}$ tissue. Uterine and ovarian tungsten concentrations were not reported.

Autoradiography of whole pregnant mice injected on Day 8 of gestation showed ${ }^{185} \mathrm{~W}$ concentration to be high in the ovarian follicles at $48 \mathrm{~h}$ post-injection, but not in corpora lutea (Wide, Danielsson, and Denker 1986).

Human levels in soft tissue including the placenta corresponding to doses including the $0.005 \mathrm{mg} / \mathrm{kg}$ used in the Nadeenko studies are listed in Table F.6.

\section{F.2.2.3 Evaluation and Identification of Data Gaps}

Overall, it is of high importance to determine whether tungsten exposure has the potential to disrupt reproductive function either in the male or female or both. If real, effects of tungsten on reproduction would be of significantly more importance than the nephrotoxic effects of uranium in view of the small minority of individuals with compromised renal function for whom the effects would be a concern.

Tungsten has been observed to have in vivo embryotoxic effects in mice exposed acutely (Wide 1984) and rats given chronic peroral exposure (Nadeenko and Lenchenko 1977, Nadeenko et al 1977, 1978). Wide, Danielsson and Dencker (1986) also induced embryotoxic effects in vitro, showing that tungsten inhibited chondrogenesis in chick embryo limb bud cells at concentrations equivalent to those causing increased fetal resorption in vivo.

Tungstate has the potential to interfere with the pituitary-gonadal hormonal control system via effects on ovarian adenyl cyclase. It may also interfere with progesterone support of the uterine environment for pregnancy maintenance via blocking of progesterone receptors. Long-term exposure of mice to 
tungsten in drinking water was shown to lower progesterone levels in pregnancy, although quantitative data were not reported.

Thus, rodent studies and in vitro experiments on enzyme systems and steroid hormone receptors suggest strongly that tungsten has the potential to interfere with reproduction both by exerting direct embryo- or fetotoxic effects and possibly also by disrupting maternal hormonal systems crucial to the normal maintenance of pregnancy. Whether these effects extend to humans is not known.

Furthermore, one animal study suggests potential effects on male fertility. A study by Idiatullina (1981) reported decreased sperm motility in rats (to about $90 \%$ of control value) in response to subchronic inhalation exposure. The decreased motility was accompanied by "weakly expressed hyperemic vessels" observed histologically in the testes which was more pronounced in the $1.0 \mathrm{mg} / \mathrm{m}^{3}$ exposure group than in the $0.5 \mathrm{mg} / \mathrm{m}^{3}$ group. A report by Gunnison et al (1987), however, indicated that tungsten at levels too low to reduce sulfite oxidase activity had no effect on testicular weight or histology. The differences in results between the Gunnison et al (1987) study and that of Idiatullina (1981) may be related in part to strain differences, different routes of exposure and other treatment differences, and possible differences in histochemical techniques used to prepare the testes for microscopic examination.

Thus, tungsten may have potentially deleterious effects on human male reproductive function. If this is true at environmentally relevant exposure levels and routes, the implications are of concern for the general population in view of changes seen in recent years in male fertility. Working (1988) indicated that there is little excess in sperm numbers necessary for fertility in humans compared to animals, especially the rat. Carlsen et al (1992) presented evidence of a 50\% decrease in sperm counts over the past 50 years in humans based on an evaluation of papers from around the world. Sharpe and Skakkebaek (1993) attribute falling sperm counts in human males over this time period to environmental estrogens and/or other environmental contaminants. The downward shift in the distribution of sperm counts puts a greater percentage of the male population into the ranges of relative and absolute infertility. Any further influence such as tungsten that decreased sperm quality or quantity would thus have the potential to influence fertility significantly, particularly in subfertile males.

\section{F.2.2.4 Tentative Dose-Response Relationship}

The in vivo studies of Nadeenko and colleagues (Nadeenko and Lenchenko 1977; Nadeenko et al $1977,1978)$ tested only one dose of tungsten $(0.005 \mathrm{mg} / \mathrm{kg})$ so no information is available from these reports regarding dose-response relationships. Likewise, the in vivo studies of Wide (1984) and Wide, Danielsson, and Denker (1986) used only one dose. However, the in vitro portion of the latter study demonstrated dose-related inhibition of chick limb bud cartilage by tungstate.

Idiatullina's (1981) observation of tungstate inhibition of sperm motility implies a possible threshold and the response appears to behave as a step function rather than showing dose-related increments. No effect was seen at $0.1 \mathrm{mg} / \mathrm{m}^{3}$; the responses at 0.5 and $1.0 \mathrm{mg} / \mathrm{m}^{3}$ were 10 and $12 \%$ inhibition or essentially equivalent.

The indirect effects of tungsten on testicular atrophy and spermatogenesis inhibition appear to have been dose related. These effects were observed in rats made sulfite oxidase-deficient (Gunnison et al 1987 ) by tungstate in drinking water coupled with a low molybdenum diet. The testicular damage was 
seen in $17 \%$ of animals drinking water at 400 ppm tungstate and $90 \%$ of rats at 800 ppm tungstate and was attributed to the buildup of endogenous sulfite levels.

\section{F.2.2.5 Research Recommendations}

It is essential to verify the results of Nadeenko and colleagues and those of Wide and colleagues on embryotoxicity and to determine more specifically whether the effects of tungsten are exerted directly on the embryo or on the maternal environment. Furthermore, the observations of Idiatullina (1981) suggest effects on the male reproductive system.

In order to verify the possible effects of tungsten on reproductive endpoints and at the same time distinguish reproductive effects from possible genetic effects (dominant lethal mutations) it is recommended that a dominant lethal experiment be performed in male mice, a total reproductive capacity test be performed in females, and if any effects are observed with short-term exposures at the maximum tolerated dose (MTD), a dose-response experiment may be indicated. A long-term low-dose experiment on total reproduction in females should also be considered if responses are seen in the initial tests, with measurement of circulating progesterone performed at sacrifice as well as tungsten determinations in selected tissues and blood if possible. Histopathology on selected tissues may be warranted. These experiments can be designed to distinguish embryotoxic or genotoxic effects from effects on the maternal environment that interfere with normal support of pregnancy.

If significant effects are observed in mouse experiments, consideration would be warranted of studying reproductive effects in a larger (nonrodent) species.

\section{F.2.3 Teratogenicity}

\section{F.2.3.1 Description of Effects}

Nadeenko and Lenchenko (1977) observed that ossification of several bones, especially the pubis, was delayed or inhibited in fetuses of rats exposed perorally for 8 months to tungsten $(0.005 \mathrm{mg} / \mathrm{kg})$; molybdenum at $0.025 \mathrm{mg} / \mathrm{kg}$ had a similar but not quite as pronounced an effect on ossification. The results of these and other studies bearing on the possible developmental toxicity of tungsten are summarized in Table F.8.

Nadeenko et al (1978) likewise reported that peroral exposure of rats for 7 months to tungsten (form not specified, but probably sodium tungstate) at a dose of $0.005 \mathrm{mg}$ W/kg resulted in decreased skeletal ossification in fetuses as well as increased fetal resorption. Detailed microscopic investigation showed an expansion of the brain ventricles, calyx of the kidney, and the bladder in a few cases (quantitative data not given); no such changes were seen in the controls, so the authors concluded that these effects were likely due to the tungsten exposure.

Chiang et al (1981 Abstract) failed to observe any evidence of external, soft tissue, or skeletal abnormalities in pups from Wistar rats made sulfite oxidase-deficient by a high tungsten/low molybdenum regimen for 4 weeks followed by 3 weeks of sulfite administration at 3 dose levels prior to mating and during gestation. Doses of tungsten and molybdenum were not specified but were sufficient to reduce hepatic sulfite oxidase to about $1-2 \%$ that of normal adult rats, based on their previous work (Gunnison et al 1987). 
Table F.8. Developmental toxicity of tungsten and molybdenum

\begin{tabular}{|c|c|c|c|c|c|}
\hline $\begin{array}{l}\text { Species/ } \\
\text { compound }\end{array}$ & Route & Dose & $\begin{array}{l}\text { Exposure } \\
\text { duration }\end{array}$ & Endpoints observed & References \\
\hline Rat/tungsten & Oral & $0.005 \mathrm{mg} / \mathrm{kg}$ & $\begin{array}{l}\text { Chronic, } \\
8 \text { months }\end{array}$ & Increased embryotoxicity & $\begin{array}{l}\text { Nadeenko and } \\
\text { Lenchenko } \\
1977\end{array}$ \\
\hline Rat/molybdenum & Oral & 0.025 & $\begin{array}{l}\text { Chronic, } \\
8 \text { months }\end{array}$ & Increased embryotoxicity & $\begin{array}{l}\text { Nadeenko and } \\
\text { Lenchenko } \\
1977\end{array}$ \\
\hline Rat/tungsten & Oral & $0.005 \mathrm{mg} / \mathrm{kg}$ & $\begin{array}{l}\text { Chronic, } \\
8 \text { months }\end{array}$ & $\begin{array}{l}\text { Increased } \\
\text { embryotoxicity; } \\
\text { decreased skeletal } \\
\text { ossification }\end{array}$ & $\begin{array}{l}\text { Nadeenko et al } \\
1977\end{array}$ \\
\hline $\begin{array}{l}\text { Rat/sodium } \\
\text { tungstate }\end{array}$ & Oral & $0.005 \mathrm{mg} / \mathrm{kg}$ & $\begin{array}{l}\text { Chronic, } \\
7 \text { months }\end{array}$ & $\begin{array}{l}\text { Increased } \\
\text { preimplantation } \\
\text { embryotoxicity; } \\
\text { decreased skeletal } \\
\text { ossification }\end{array}$ & $\begin{array}{l}\text { Nadeenko et al } \\
1978 \text {; same } \\
\text { data as N\&L } \\
1977 \text { (more } \\
\text { detailed) }\end{array}$ \\
\hline $\begin{array}{l}\text { Rat/sodium } \\
\text { molybdate }\end{array}$ & Oral & $0.025 \mathrm{mg} / \mathrm{kg}$ & $\begin{array}{l}\text { Chronic, } \\
7 \text { months }\end{array}$ & $\begin{array}{l}\text { Increased } \\
\text { embryotoxicity; } \\
\text { decreased skeletal } \\
\text { ossification }\end{array}$ & $\begin{array}{l}\text { Nadeenko et al } \\
1978 \text {; same } \\
\text { data as N\&L } \\
1977 \text { (more } \\
\text { detailed) }\end{array}$ \\
\hline $\begin{array}{l}\text { Mouse/sodium } \\
\text { tungstate }\end{array}$ & $I^{a}$ & $\begin{array}{l}0.1 \mathrm{~mL} \text { of } 25 \mathrm{mM} \\
\text { sol'n }(18.4 \mathrm{mg} \\
\mathrm{W} / \mathrm{kg} \text { maternal } \\
\text { body wt) }\end{array}$ & $\begin{array}{l}1 \text { injec @d 3, } \\
\text { or } \\
1 \text { injec @d } 8\end{array}$ & $\begin{array}{l}\text { Increased fetal } \\
\text { resorption; no } \\
\text { developmental effects }\end{array}$ & Wide 1984 \\
\hline $\begin{array}{l}\text { Mouse/sodium } \\
\text { molybdate }\end{array}$ & IV & $\begin{array}{l}0.1 \mathrm{~mL} \text { of } 100 \mathrm{mM} \\
\text { sol'n ( } 38.4 \mathrm{mg} \\
\text { Mo/kg maternal } \\
\text { body wt) }\end{array}$ & $\begin{array}{l}1 \text { injec @ d } 3 \text {, } \\
\text { or } \\
1 \text { injec @ d } 8\end{array}$ & $\begin{array}{l}\text { Decreased skeletal } \\
\text { ossification; decreased } \\
\text { fetal weight gain; no } \\
\text { effect on resorption }\end{array}$ & Wide 1984 \\
\hline
\end{tabular}

${ }^{a}$ Abbreviation: IV $=$ intravenous.

Wide (1984) failed to observe any increase in developmental abnormalities in fetuses of mice treated acutely with large IV doses of sodium tungstate (dose equivalent to $18.4 \mathrm{mg} \mathrm{W} / \mathrm{kg}$ ) on Day 3 or Day 8 of gestation. The percentage of fetuses with immature skeletons was increased, but not to a level of statistical significance. Molybdenum did significantly inhibit skeletal ossification in this study. An increased rate of resorption was associated with tungstate treatment as described in Sect. F.2.2.

Wide, Danielsson, and Denker (1986) treated chick mesenchymal limb bud cells in vitro with sodium tungstate and observed an inhibition of chondrogenesis formation by these cells at concentrations in the range corresponding to those in mouse fetuses in which resorption was increased. Cohen, Johnson, and Rajagopalan (1974) comment without presenting data that when female rats are given dietary tungsten treatment starting immediately after breeding (apparently sufficient to produce molybdenum deficiency), the offspring are "normal by all criteria." Their procedure was to feed a diet containing 
$0.7 \mathrm{~g}$ sodium tungstate per $\mathrm{kg}$ feed ( $400 \mathrm{ppm}$ tungsten). Whether or how rigorously the pups were examined for internal developmental abnormalities is not known.

\section{F.2.3.2 Relationship to Biokinetic Model}

The same information relating to the work of Nadeenko and Lenchenko (1977), Nadeenko et al (1978), and Wide, Danielsson, and Denker (1986) as discussed above with respect to reproductive effects holds true here.

\section{F.2.3.3 Evaluation and Identification of Data Gaps}

The work of Nadeenko and colleagues is very suggestive of developmental toxicity associated with chronic low-dose exposure to tungsten. The observation by Wide, Danielsson, and Denker (1986) of tungsten accumulating in the fetus and preferentially in neural tissue, later in skeletal tissue, at certain times in fetal development is also strongly suggestive of the possibility of tungsten effects on the embryo and fetus. The failure of Wide (1984) to observe a significant decrease in skeletal ossification or chondrogenesis may be due to species differences or to major differences in the route and quantity of tungstate administered from the experiments reported by Nadeenko et al $(1977,1978)$ and Nadeenko and Lenchenko (1977).

The in vitro work of Wide, Danielsson, and Denker (1986) is consistent both with a fetotoxic effect and with the possibility that tungsten may induce the type of developmental abnormality (delayed ossification) described by Nadeenko et al $(1977,1978)$ and Nadeenko and Lenchenko (1977).

Nadeenko et al (1978) also observed teratogenic effects on rat brain, kidney, and bladder that were likely due to their extremely low dose chronic tungstate exposure regimen.

Further work would be highly desirable to replicate the results of Nadeenko and colleagues; in the absence of more data, it can only be said that the existing literature is suggestive of tungsten having the potential to induce developmental abnormalities in mammals. The observation of neurotoxicity in adult animals, brain abnormalities in rat fetuses in chronic experiments (Nadeenko et al 1978), and preferential tungsten accumulation in fetal neural tissue (Wide, Danielsson, and Denker(1986) also raises the issue of developmental neurotoxicity which has not been evaluated experimentally in any previous work.

It is essential to resolve the question of developmental toxicity with regard to long-term, low-dose oral tungsten exposure before embarking on any activity with the potential to expose humans in the general population to tungsten. Developmental toxicity associated with tungsten, if confirmed, would be of much greater significance than the nephrotoxicity known to be associated with low-dose uranium exposure, in part because the latter is of concern only to the minority of individuals lacking a kidney or with severely compromised kidney function.

\section{F.2.3.4 Tentative Dose-Response Relationship}

The studies of Nadeenko and Lenchenko (1977) and Nadeenko et al (1978) give no information on possible dose-response relationships with regard to the developmental toxicity they observed in rat embryos as they used only one dose of tungsten $(0.005 \mathrm{mg} / \mathrm{kg}$ body weight. Wide, Danielsson, and Denker (1986) found a dose-response relationship in the tungstate-induced inhibition of chick limb bud chondrogenesis in vitro. No threshold was observed at the concentrations tested. 


\section{F.2.3.5 Research Recommendations}

To verify the reported developmental toxicity effects, it is recommended that an assessment of fetal development (examination for teratogenic effects) be made after exposure of female mice to tungsten prior to and during gestation. This could be performed in conjunction with the reproductive toxicity studies recommended above.

To investigate the possibility of developmental neurotoxicity, it is recommended that a preliminary study in rats be performed in which dams are exposed to relatively high doses of sodium tungstate for a short time ( 3 to 4 weeks) prior to mating, with exposure continuing during gestation and nursing. The pups should then be reared without further tungsten exposure to young adulthood (sexual maturity) and tested for normal behavior/ activity/reflexes and learning capabilities (acquisition and extinction of tasks). If effects are observed, then a study involving chronic or subchronic low-dose exposure of the dams should be pursued. In both cases, blood and brain $\mathrm{ChE}$ levels and tungsten tissue distribution as well as histopathological examination of brain tissue at sacrifice should be included in the study. If significant effects are seen in mice and rats, experiments in a larger species should be seriously considered.

\section{F.2.4 Growth and Nutrition}

\section{F.2.4.1 Molybdenum Displacement (sulfite oxidase and xanthine oxidase)}

Three molybdenum-containing enzyme systems have been described in animals; xanthine oxidase (XO) (also referred to as xanthine dehydrogenase), aldehyde oxidase (AO) (also referred to as aldehyde dehydrogenase), and sulfite oxidase (SO) (Johnson and Rajagopalan 1974). Molybdenum centers of $\mathrm{XO}$ and related enzymes are the sites where the oxidative hydroxylation of substrates is accomplished (Johnson, Waud, et al 1974). Hence, molybdenum is an integral prosthetic group for both SO and XO (Johnson Cohen and Rajagopalan 1974 Johnson, Waud, et al 1974). However, results from Johnson, Waud et al (1974) established the fact that XO apoenzyme present in livers of tungsten-treated rats is functionally competent in activities not dependent on the presence of molybdenum.

Aldehyde oxidase (AO) is a molybdoprotein found in the hepatic cytosol, mitochondria, and microsomes. AO oxidizes aldehydes to carboxylic acids with $\mathrm{NAD}^{+}$as the cofactor (Sipes and Gandolfi 1991). There are two types of AO; one oxidizes formaldehyde and the other oxidizes free aldehydes and has broad substrate specificity (Sipes and Gandolfi 1991, Coughlan 1980). The latter is involved in most xenobiotic aldehyde oxidation. Genetic deficiencies of AO cause the accumulation of acetaldehyde after ethanol ingestion. As the concentration of the aldehyde increases, it is more likely to react with nucleophiles such as endogenous amines (Sipes and Gandolfi 1991). Presumably AO responds in the same manner as $\mathrm{SO}$ and $\mathrm{XO}$ to $\mathrm{W}$ exposures; however, no information regarding $\mathrm{AO}$ response to tungsten was found. Hence, $A O$ is not discussed further in this review.

Sulfite oxidase or sulfite: cytochrome $\mathrm{C}$ oxidoreductase, a mitochondrial molybdohemoprotein (Johnson and Rajagopalan 1974) located in the intermembrane compartment (Cohen, Johnson, and Rajagopalan 1974), catalyzes the terminal step in the oxidative metabolism of the sulfur moiety of sulfur amino acids (Cohen, Johnson, and Rajagopalan 1974). Congenital SO deficiency indicates that SO is essential in vivo (Mudd, Irreverre, and Laster 1967, Shih et al 1977). Liver SO has a long halflife of 4.7 days with first order kinetics when rats are treated with $100 \mathrm{ppm}$ tungsten (Cohen et al 1973). Under the same conditions, the half-lives of kidney and ileum SO are 4.2 and 1.3 days, 
respectively (Johnson and Rajagopalan 1974). Human liver SO concentration is 5-10\% that of rat liver SO concentration (Johnson and Rajagopalan 1976a).

Xanthine oxidase is a cytoplasmic molybdoprotein (Johnson and Rajagopalan 1974) involved in the terminal metabolism of free purine bases (xanthine, hypoxanthine, etc) to form uric acid (Cohen, Johnson, and Rajagopalan 1974). Protein is necessary for XO synthesis and can influence the concentration of XO available to break down free purine bases (Rowe and Wyngarden 1966, Higgins 1956). Xanthine oxidase in the liver has a half-life of 2.9 days with first order kinetics when rats are treated with $100 \mathrm{ppm}$ tungsten (Johnson and Rajagopalan 1974). Under the same conditions, the halflives of $\mathrm{XO}$ in the kidney and ileum are 1.4 and 1.3 days, respectively (Johnson and Rajagopalan 1974).

Animals can be made deficient in $\mathrm{SO}$ and $\mathrm{XO}$ by a high tungsten/low molybdenum regimen maintained over a period of days. Administration of tungsten to rats maintained on a low molybdenum diet $(20-30 \mu \mathrm{g} \mathrm{Mo} / \mathrm{kg}$ diet) resulted in dose and time-dependent losses of SO and XO activities and hepatic molybdenum (Cohen et al 1973). Changes in dietary molybdenum intakes are reflected in changes in the levels of molybdenum in the tissues (Underwood 1971), i.e., there appears to be little, if any, molybdenum storage in body tissues.

Rats fed high tungsten/low molybdenum diets continue to produce the inactive forms of SO and XO. In XO, the apoenzyme was devoid of either molybdenum or tungsten (Johnson et al 1974). In SO, as many as $35 \%$ of the enzyme molecules were seen to contain tungsten (Johnson, Cohen, and Rajagopalan 1974). Molybdenum-free SO is inactive, whether it exists as the apomolecule or has tungsten incorporated (Johnson, Cohen, and Rajagopalan 1974). The return of enzyme activity results from the incorporation of molybdenum into pre-existing inactive molecules (Johnson and Rajagopalan 1974). It would appear that the systems for the absorption and transport of molybdenum, presumably as molybdate, are sufficiently nonspecific to accept tungsten (as tungstate) in place of molybdenum. However, the fact that $1 \mathrm{ppm}$ molybdenum could almost completely overcome the effect of $100 \mathrm{ppm}$ tungsten indicates that the processes involved in molybdenum uptake or utilization, or both, have a greater affinity for molybdenum than for tungsten. This is further substantiated by the inability of 100 ppm of tungsten to affect either hepatic enzyme activity when administered for up to 5 weeks to rats fed normal rat chow which has a molybdenum content of $1000 \mu \mathrm{g} / \mathrm{kg}$ (Johnson and Rajagopalan 1974).

\section{Possible dose-response relationship}

In rats and from the few case reports of human SO deficiency,a linear relationship appears to hold between the amount of SO activity and the normal function of animals and humans after reaching a threshold. This threshold has not been determined for humans. Rats experience a change in organic sulfur urine out put at SO activity levels of $7 \%$ of control rats, but rats function normally with SO activity levels at $1 \%$ of control rats (Gunnison et al 1980 pp. 209-20). However, rat SO values were obtained using molybdenum-deficient diets with tungsten in drinking water. After reaching a steady state SO activity level of $1 \%$, rats contained approximately $0.008 \mathrm{mg}$ of tungsten in their total liver and may be between $0.002-0.04 \mathrm{mg}$ of tungsten (with an uncertainty factor of 5) by using the biokinetic model developed for this project. The most applicable scenario for this type of effect in humans would be to those people who are SO deficient. Presumably people can function normally with SO concentrations at 32-50\% of normal (Shih et al 1977). It is assumed that these people would be susceptible to tungsten only when molybdenum concentrations in the diet are below normal since 
the affinity for SO apoenzyme is much greater for molybdenum than tungsten. If the mechanisms of tungsten-molybdenum interactions in rats hold true for humans, this route of tungsten toxicity in humans would occur in no more than $10 \%$ of the population and possibly could be combatted by supplementing the diet with additional molybdenum.

\section{Tungsten effects on sulfite oxidase and xanthine oxidase activities}

Animals. Rats maintained on low molybdenum diets $(20-30 \mu \mathrm{g} / \mathrm{kg}$ diet) and tungsten as sodium tungstate, supplemented water at 4,25 , or $40 \mathrm{ppm}$ for 3 weeks showed a decrease in hepatic SO and XO content from approximately 45 to $20 \%$ of the control values and molybdenum content from approximately 80 to $20 \%$ of the control values (Cohen et al 1973, Johnson and Rajagopalan 1974). By giving drinking water from $200 \mathrm{mg} / \mathrm{kg}$ tungsten as sodium tungstate for 28 days and then lowering tungsten concentration to $100-120 \mathrm{mg} / \mathrm{kg}$ tungsten, Gunnison et al (1981a) were able to maintain SO activity at $1 \%$ of the mean adult rat control levels. Maintenance of this low SO activity level occurred with an approximate W:Mo ratio of $3100: 1$. Rats injected daily intraperitoneally with $10 \mathrm{mg} / \mathrm{kg}$ tungsten for 3 weeks only showed progressive decrease in SO activity (Johnson and Rajagopalan 1974). Rats maintained on 25 ppm tungsten in drinking water did not differ from the control rats in any observable physical criteria. No significant difference in body weight, liver weight, liver protein content, or lung weight was found. Even at $100 \mathrm{ppm}$ tungsten the animals showed no toxic signs or symptoms (Johnson and Rajagopalan 1974). Rats exposed to $45 \mathrm{mg} \mathrm{W} / \mathrm{kg}$ diet as sodium tungstate $(0.02 \mathrm{mg} \mathrm{Mo} / \mathrm{kg}$ ) for 6 weeks had essentially no measurable XO activity (Higgins, Richert, and Westerfield 1956b). No deaths occurred during the 12 weeks that the rats were maintained on the dietary regimens of 45 or $94 \mathrm{mg} \mathrm{W} / \mathrm{kg}$ diet with 0.02 or $60 \mathrm{mg} \mathrm{Mo} / \mathrm{kg}$ diet (Higgins, Richert, and Westerfield 1956b). In another study, serum uric acid decreased and serum xanthine increased with increasing tungstate dose (up to $62 \mathrm{ppm} \mathrm{W}$ for 10 days) and thyroid XO was significantly decreased (Kawada et al 1982). Rats exposed to the $94 \mathrm{mg} / \mathrm{kg}$ tungsten diet excreted $0.42 \mathrm{mg}$ of xanthine per day as compared with $0.1 \mathrm{mg}$ for the control groups (Higgins, Richert, and Westerfield 1956b). Rats fed tungsten in a 1000 or 2000:1 ratio of W:Mo grew normally and oxidized xanthine to uric acid and allantoin as well as the controls in spite of the fact that all tissues were depleted of molybdenum and $\mathrm{XO}$; the tissue changes were reversed by additional dietary molybdenum (Higgins, Richert, and Westerfield $1956 \mathrm{~b}$ ). When rats were fed concentrations of tungsten at $400 \mathrm{mg} / \mathrm{kg}$ on low molybdenum diets ( $35 \mathrm{Mo} \mu \mathrm{g} / \mathrm{kg}$ ) for up to 21 weeks, toxic effects were seen in some rats (Gunnison et al 1987). Rats fed the same low molybdenum diet at $800 \mathrm{mg} / \mathrm{kg}$ tungsten for up to 21 weeks began to show toxic signs at 5 weeks. These symptoms include decreased growth rate, chromodacryorrhea (evidence suggesting heme in the urine), waxy coating on tail, small ulcerations and nodules on the tail, and occasional sores on other parts of the body. This toxicity was not specifically related to the tungsten but was attributed to the build up of endogenous sulfite as a result of the non-existent $S O$ concentration. Values of 400 and $800 \mathrm{mg}$ W/kg per day give an approximate W:Mo ratio of 11,400 and 22,900 to 1 , respectively. Gunnison et al (1988) stated that $400 \mathrm{mg} \mathrm{W} / \mathrm{kg}$ with their low molybdenum diet resulted in severe deficiency of SO without overt acute toxicity.

The effect of tungsten on $\mathrm{XO}$ in goats has also been observed. The ingestion of sodium tungstate by young goats at $28 \mathrm{~g} \mathrm{~W} / \mathrm{kg}$ feed for 3 or 5 months diminished the amount of XO in their livers to 29 and $20 \%$ of the controls, respectively (Owen and Proudfoot 1968). No other effects were noted.

In chickens, liver and other tissue molybdenum concentrations were $<10 \%$ of normal values, while XO activity in the small intestine, liver, kidney and pancreas were reduced to $<15 \%$ of normal by 4.5 and $9.4 \mathrm{mg} \% \mathrm{~W}$-diets as sodium tungstate for 5 weeks. Normal values were restored, by adding 2 and 
$6 \mathrm{mg} \% \mathrm{Mo}$ ( 20 and $60 \mathrm{mg} \mathrm{Mo} / \mathrm{kg}$ ), respectively, as molybdate to the diets containing tungstate; except that with the $9.4 \% \mathrm{~W}$ diet, growth was still depressed and mortality was $9 \%$ (Higgins, Richert, and Westerfield 1956b).

Sulfite oxidase is instrumental in counteracting the toxic systemic effects of bisulfite. Animals deficient in sulfite oxidase are much more sensitive to bisulfite toxicity than controls. Rats maintained on drinking water with $100 \mathrm{ppm}$ tungsten for 3 or 5 weeks and on a low molybdenum diet experienced a lower bisulfite $\mathrm{LD}_{50}(\mathrm{mg} / \mathrm{kg})$ at $49 \%$ and $32 \%$ of the control rats fed rat chow, respectively (Cohen et al 1973).

Humans. A child with SO activity of $<5 \%$ of normal childhood levels experienced normal development until 17 months. When SO deficiency was discovered at 4 yrs, the child had permanent mental deficits, dislocated lenses and hemiplegia (Shih et al 1977). In fact, patients with severe congenital SO deficiency suffer from mental retardation, lens dislocation and early death (Cabre et al 1990). Less severe cases can result in intolerance to some sulfite-containing foods (Cabre et al 1990). Data indicate that SO is essential in vivo (Mudd, Irreverre, and Laster 1967, Shih et al 1977). Persons with SO activity at $30-47 \%$ of control levels appeared normal (Shih et al 1977). Normal SO from human autopsy livers ranges from 10-25 U/g wet weight tissue (Johnson and Rajagopalan 1976a). It has been estimated that 6 out of every 100 people have some degree of sensitivity to sulfite ion (Markley, Meloan, and Lambert 1986) presumably due to low SO levels. About 30\% of these reactions occurred in non-asthmatics with no known allergies (Hecht and Willis 1983). Approximately $10 \%$ of asthmatic patients are at risk for developing sulfite reactions (Settipane 1986). In persons who are sensitive to sulfites, reactions occur within minutes of exposure to sulfites and range from difficulty in breathing to convulsions; in extreme cases, loss of consciousness, and in rare cases, death occur (Markley, Meloan, and Lambert 1986). In a case report of food sensitivity to sulfites, sensitivity was tested by dose. Oral exposure to $5 \mathrm{mg}$ of sulfite caused a decrease in forced expiratory volume in 1 second $\left(\mathrm{FEV}_{1}\right)$ while decreases in maximum midexpiratory flow rate (MMRF) occurred with exposure to $0.5,1$ and $5 \mathrm{mg}$ sulfite (Twarog and Leung 1982). No changes in $\mathrm{FEV}_{1}$ or MMFR were noted at $0.1 \mathrm{mg}$ sulfite (Twarog and Leung 1982).

Congenital deficiency of XO has been described in humans and does not cause serious health impairment (Watts et al 1964). Individuals with XO deficiency experience mild myopathy from the deposition of hypoxanthine and xanthine crystals in muscle, and formation of renal xanthine calculi (Coughlan 1980).

\section{Evaluation of significance and data gaps}

Tungsten decreases hepatic XO, SO, and molybdenum content in a time- and dose-dependent manner. Xanthine oxidase appears to be non-essential to the maintenance of life. Data based on chickens may not accurately reflect XO mechanisms in humans. (see Growth effects section in this Appendix) Rats and goats seem to function well with XO concentrations near $20 \%$ of normal levels in the liver. This may indicate that sufficient quantities of purine wastes are eliminated directly. Data indicate that, at least in rats, xanthine can be excreted directly (Kawada et al 1982 and Higgins, Richert, and Westerfield 1956a). Congenital deficiency of XO in humans reveals no serious health impairment for humans (Watts et al 1964). Hence, any effect tungsten would have on XO should not produce serious consequences from the depletion of this enzyme. 
The effects of tungsten on SO appear to be more serious than tungsten effects on XO. Data on rats presented by Cohen et al (1973) and Gunnison et al (1987) suggest that the concentration of SO is more important in the elimination of sulfur wastes created by the body. It can be theorized based on Gunnison et al (1987) that the toxicity produced by high concentrations of tungsten in the diet result from the increase in endogenous sulfite due to the elimination of SO. Data from human cases of SO deficiency indicate that SO is essential in vivo (Mudd, Irreverre, and Laster 1967, Shih et al 1977) and that minor decreases in SO can be tolerated if foods high is sulfite are avoided (Cabre et al 1990, Twarog and Leung 1982). However, no determination has been made concerning the concentration of sulfur or tungsten necessary to produce toxic effects when there is a SO deficiency. If tungsten concentrates in mitochondrial-rich tissues (i.e., liver) in humans, the effect of concentrating tungsten could render the few remaining SO enzymes non-functional in SO-deficient individuals. This depletion of SO, with exposure to a high sulfite diet, could produce breathing difficulty, loss of consciousness, or even death.

\section{Research recommendations}

In persons with low SO activity levels (i.e., persons with know food sulfur sensitivity) diets with known sulfur content could be used to measure sulfur metabolites in the blood and urine. This may aid in the determination of the relationship between the amount of sulfur and the amount of SO necessary to metabolize organic sulfur. Furthermore, these data could be used in conjunction with tungsten distribution data to determine the amount of tungsten necessary to produce SO deficiency in humans. It would be helpful to examine human liver biopsy samples for the presence of molybdenum, tungsten, and SO in order to determine the relationship between these compounds in humans. Storage and transport of liver biopsy tissues necessitate immediate freezing at $-50^{\circ} \mathrm{C}$ in order to preserve the SO for analysis (Johnson and Rajagopalan 1796a). However, access to liver biopsy tissue may be difficult to obtain. An alternative experiment could examine liver concentrations of molybdenum, tungsten, and SO after a subacute study in rats as suggested in the Neurotoxicity section of this Appendix. This information could then be used in conjunction with the available literature to relate SO tissue concentration to tungsten concentration. Although rats may not be the best animal for determination of tungsten effects on SO (Daniel 1986), animals that would be more suitable for SO analysis (pigs or dogs) in relation to tungsten effects on humans might be cost prohibitive.

\section{F.2.4.2 Growth Effects}

Weanling rats fed diets of 45 or $94 \mathrm{mg}$ W/ $\mathrm{kg}$ with low molybdenum for 12 weeks did not experience depressed growth rates or mortality (Higgins, Richert, and Westerfield 1956a). Rats fed tungsten as sodium tungstate in a 1000 or $2000: 1$ ratio of W:Mo grew normally in spite of the fact that all tissues were depleted of molybdenum and $\mathrm{XO}$; the tissue changes were reversed by additional dietary molybdenum (Higgins, Richert, and Westerfield 1956a). Male rats with drinking water exposure of 50, 100,200 , or $400 \mathrm{mg} / \mathrm{kg}$ tungsten as sodium tungstate with a low molybdenum diet for 21 weeks exhibited weight gain and survival similar to rats on the control diet (Gunnison et al 1987). However, when exposure occurred at $800 \mathrm{mg} / \mathrm{kg}$ tungsten with a W:Mo ratio of $22,900: 1$, rats experienced statistically significant decreases in the rate of weight gain beginning between week 4 and 8 and continuing throughout the treatment period (Gunnison et al 1987). Rats in the $800 \mathrm{mg} \mathrm{W} / \mathrm{kg}$ treatment group also experienced a $30 \%$ mortality (Gunnison et al 1987). When sodium tungstate was given to rats at a concentration of $5 \mathrm{mg} \mathrm{W} / \mathrm{kg}$ in drinking water for a lifetime, no effect on growth rate was noted. A slight increase in mortality was observed which was significant only in males (Schroeder and Mitchner 1975). 
In subacute experiments in albino rats and rabbits, Nadeenko (1966) exposed the animals orally to $100,40,25$ and $10 \mathrm{mg} / \mathrm{kg}$ sodium tungstate (frequency of dosing was unspecified, but probably daily) for an unspecified time period (probably $\leq 30$ days). These doses were sufficient to retard growth in all rat groups. Albino rats given lower doses of $0.5,0.05$ and $0.005 \mathrm{mg} \mathrm{W} / \mathrm{kg}$ for 7 months as sodium tungstate experienced no significant deviations in weights.

Idiatullina (1981) reported the results of inhalation exposure of rats to sodium tungstate as a highly dispersed powder. Acute/subacute exposure to high levels apparently had no statistically significant effect on growth, but subchronic (4 months) exposure to $1 \mathrm{mg} / \mathrm{m}^{3}$ depressed growth somewhat (to $91 \%$ of control value, $\mathrm{P}<0.05$ ) while 0.5 and $0.1 \mathrm{mg} / \mathrm{m}^{3}$ had no effect.

In chickens, sodium tungstate fed to dams at $500 \mathrm{ppm}$ tungsten) at normal molybdenum levels caused significant depression in weight gain in chicks for at least 20 days (Teekel and Watts 1959a, 1959b). Chicks fed low molybdenum feed $(0.02 \mathrm{mg} \mathrm{Mo} / \mathrm{kg})$ containing either 4.5 or $9.4 \mathrm{mg} \%$ tungsten as sodium tungstate developed molybdenum deficiency. Growth rates were depressed 8 and 19\% respectively at 5 weeks (Higgins, Richert, and Westerfield 1956b). Mortality at 3 weeks was 14 and $24 \%$, respectively and 24 and $28 \%$ at 5 weeks (Higgins, Richert, and Westerfield 1956a). Chicks fed $45 \mathrm{mg} \mathrm{W} / \mathrm{kg}$ plus an additional $2 \mathrm{mg} \mathrm{Mo} / \mathrm{kg}$ experienced $9 \%$ mortality at 5 weeks (Higgins, Richert, and Westerfield 1956a). Both diets produced decreased levels of $\mathrm{XO}$ and molybdenum in tissues. Normal enzyme and molybdenum concentrations were restored, by adding 2 and $6 \mathrm{mg} \% \mathrm{Mo}$ (20 and $60 \mathrm{mg} \mathrm{Mo} / \mathrm{kg}$ ), respectively, as molybdate to the diets containing tungstate. However, with the 9.4 $\mathrm{mg} \% \mathrm{~W}$-diet, growth was still depressed $4 \%$ and mortality was $9 \%$ (Higgins, Richert, and Westerfield 1956b).

\section{Evaluation of significance and identification of data gaps}

Data for rats are conflicting in that Higgins, Richert, and Westerfield (1956a) and Gunnison et al (1987) reported no growth effects with exposures of 45 to $400 \mathrm{mg} \mathrm{W} / \mathrm{kg}$ of diet or of water as sodium tungstate for exposure periods from 12 to 21 weeks in the diet while Nadeenko (1966) reported retarded growth for subacute exposure to concentrations as low as $10 \mathrm{mg} / \mathrm{kg}$ body weight of sodium tungstate in drinking water. If assumptions are correct with respect to dosing time for Nadeenko (1966) at $\leq 30$ days, perhaps differences in rat strains or molybdenum concentrations could be the reason for conflicting results. Chronic or subchronic exposures to low tungstate levels had no effect on growth except for a small decrease resulting from inhalation exposure (Idiatullina 1981). Nadeenko (1966) observed no significant changes in weight at sodium tungstate exposure levels of 0.005 to $0.5 \mathrm{mg} / \mathrm{kg}$ body weight for 7 months. Schroeder and Mitchner (1975) observed no growth rate effect for rats fed $5 \mathrm{mg} \mathrm{W} / \mathrm{kg}$ as sodium tungstate in drinking water. Results from Nadeenko (1966) and Schroeder and Mitchner (1975) show that low exposures of sodium tungstate had little effect on weight gain in rats. However, lifetime exposures caused a slight increase in mortality in male rats (Schroeder and Mitchner 1975).

Gunnison, Dulak, et al (1981) reported depressed weight gain in rats exposed to $800 \mathrm{mg} \mathrm{W} / \mathrm{kg}$ drinking water as sodium tungstate for 21 weeks $(\approx 5$ months). However, this dose of tungsten was indirectly and overtly toxic to the rats. The high W/low Mo exposure was thought to produce growth retardation in rats indirectly via an increased concentration of sulfites in the rats (Gunnison, Dulak, et al 1981). 
Because chickens are uricotelic, that is, animals which excrete uric acid as the final output of nitrogen metabolism, they appear to be more sensitive to disruptions of enzymatic concentrations of XO and presumably sulfite oxidase (SO) than rats and other mammals which are ureotelic and secrete urine. This sensitivity is evidenced when tungsten produces a molybdenum deficiency. Hence, data from birds may not accurately reflect the effects of tungsten which might be observed in humans.

Diet (W:Mo ratios) and exposure time may be critical factors in the determination of sublethal effects of sodium tungstate. Although low concentrations of sodium tungstate did not delay weight gain in rats, high dose subacute studies suggest that at higher concentrations, sodium tungstate ( $\geq 10 \mathrm{mg} / \mathrm{kg} / \mathrm{d}$ ) may cause retarded growth.

\section{Research recommendations}

Since no model exists for the extrapolation of growth effects data from animals to humans, examination of the effect of tungsten on the growth of animals is not recommended for this project.

\section{F.2.5 Blood Glucose Control, Carbohydrate Metabolism}

A suggestion of tungsten effects on carbohydrate metabolism in humans is found in a report by Vengerskaya and Salikhodzhaev (1962) on their study of 178 hard-metal workers. Of 37 workers in which it was evaluated, blood sugar levels were slightly elevated in 8 . The glucose tolerance curve was elevated in 8 of 14 workers tested; no data are presented, however, so it is not clear whether these were statistically significant differences from normal values. It is also not clear whether a group of matched controls was included in the study; no such group is mentioned. Thus, this report can hardly be considered definitive. The study was also confounded by the mixed exposure in the factory. The workers were exposed both to tungsten and cobalt, with tungsten air concentrations varying from 0.75 to $6.1 \mathrm{mg} / \mathrm{m}^{3}$, and cobalt from 0.6 to $3.2 \mathrm{mg} / \mathrm{m}^{3}$ (measurement methods unspecified).

Nadeenko (1966) observed that chronic oral administration of 5 and $0.5 \mathrm{mg} / \mathrm{kg}$ body weight sodium tungstate in drinking water to rabbits affected blood sugar levels by the 6 th to 7 th month of exposure. Blood sugar levels were 20 to $25 \%$ higher than control levels one hour after IV administration of galactose; at two hours after injection, uptake was essentially complete in control animals but blood levels were still $10 \mathrm{mg} \%$ in tungsten-treated rabbits. Nadeenko interpreted these results as indicating a decrease in the liver glycogen-forming capacity, although without measuring liver glycogen stores, other interpretations seem possible. Long-term administration of tungstate doses of 0.05 and 0.005 $\mathrm{mg} / \mathrm{kg}$ was ineffective in changing the response to galactose loading.

Lifetime exposure of male and female rats to $5 \mathrm{ppm}$ sodium tungstate in drinking water caused an increase in fasting serum glucose at $783 \mathrm{~d}(\mathrm{P}<0.001)$, although the effect was not consistent at all exposure times measured (Schroeder and Mitchner 1975). Male rats exposed to sodium tungstate at $0.52 \mathrm{mmol} / \mathrm{L}$ or $10.42 \mathrm{mmol} / \mathrm{L}$ for 2 months in drinking water were observed to have higher blood glucose levels $(\mathrm{P}<0.01)$ after glucose loading (mainly at 1 and $2 \mathrm{~h}$ post-loading) (Karaskova et al 1985). The liver glycogen content was significantly reduced in these rats $(P<0.01)$.

The possibility that tungsten may block liver glycogen formation is more plausible in light of its effects on glucocorticoid receptors (GR). It has been shown to bind to GR (as with progesterone receptors; see Sect. above on Reproductive Toxicity) (Murakami, Quattrociocchi, Healy, et al 1982). Like molybdate, it can block the activation of inactivated GR and also block the DNA binding of 
activated GR (Murakami, Healy, and Moudgil 1982, Moudgil et al 1983 abstr.). More recent work suggests that the tungstate effects as well as those of molybdate and vanadate on GR may be mediated by conformational stabilization of heat shock protein 90 (Hutchison et al 1992).

Tungstate also has effects on other enzymes involved with carbohydrate metabolism. Tungsten, like Mo, is known to be an effective phosphatase inhibitor (Murakami, Szocik, et al 1982). Furthermore, sodium tungstate at low concentrations stimulates the conversion of ATP to CAMP via stimulation of the adenylate cyclase system in a number of rat tissues (Hwang and Ryan 1981). Thus, low levels of W could enhance glycogenolysis or inhibit glycogen synthesis both by inhibiting phosphorylase phosphatase which catalyzes the disaggregation of active phosphorylase a, and also by stimulating the adenyl cyclase system that favors reaggregation of subunits to active phosphorylase a, the enzyme responsible for degrading glycogen in muscle and liver (White, Handler, and Smith 1964).

\section{F.2.5.1 Tentative Dose-Response Relationship}

No best guess as to the dose-response relationship in regards to tungsten exposure and blood glucose control or carbohydrate metabolism can be determined with the available literature. Human tungsten exposure data are confounded by concomitant cobalt exposure and animal tissue data cannot be directly related to normal uptake routes. Extrapolation of animal oral exposure to tungsten would yield results in excess of health effect levels that might produce neurotoxic effects in humans.

\section{F.2.5.2 Research Recommendations}

At present, the effects of tungsten on blood glucose control and carbohydrate metabolism do not appear significant and cannot be related to human exposure to tungsten alone. Hence, tungsten's effects on blood glucose control and carbohydrate metabolism do not warrant further examination under this project.

\section{F.2.6 Miscellaneous}

\section{F.2.6.1 Iron Absorption, Anemia}

Tungsten supplementation produces an effective Mo deficiency in rats, which interferes with the absorption of Fe, resulting in a lower blood hemoglobin levels ( $92 \mathrm{~g} / \mathrm{L}$ compared to normal of $93 \mathrm{~g} / \mathrm{L}$ ). A sodium tungsten supplementation of $0.39 \mathrm{~g} \mathrm{~W} / \mathrm{kg}$ feed in conjunction with feed deficient in iron reduced the ferroxidase activity of intestinal mucosal XO to $66 \%$ of its control value in Fe-deficient rats. An inhibition of apparent Fe absorption occurred with W supplementation, but after correcting for the increased gastric retention, true Fe absorption values increased $(P<0.05)$ (Shears, Neal, and Ledward 1989).

Decreased levels of hemoglobin would lead to a decrease in the oxygen content of the blood. Since studies by Shears, Neal, and Ledward (1989) were short term (weeks), it may be that the body can compensate for the decreased hemoglobin concentration produced by the sodium tungstate by increasing hemoglobin production over a longer term exposure. The intent of Shears, Neal, and Ledward (1989) was to determine the effect of W supplementation on Fe absorption. Hence, these results may only be useful in the design of further studies. 


\section{Tentative dose-response relationship}

It would not be prudent to determine a best guess as to the dose-response relationship in regard to iron absorption and anemia. Measurement of iron absorption and anemia occurred at only one exposure level. Oral exposure of $\mathrm{W}$ to rats which produced increased iron absorption and anemia was far above the exposure concentrations of $\mathrm{W}$ which produced neurological effects in rats. Because the effects of tungsten exposure in relation to iron absorption and anemia appears to be inconsequential in relation to the toxic effects of tungsten, this area of tungsten effects is not worthy of further examination under this project.

\section{F.2.6.2 Other Effects}

Tungstate stimulates various activities in tissues (Hwang and Ryan 1981, Goto et al 1992, and Fillat, Rodriguez-Gil, and Guinovart 1992), presumably because of its ability to act as an oxidant (Fillat, Rodriguez-Gil, and Guinovart 1992). Tungstate stimulates adenylate cyclase in ovarian homogenates of the rat; maximal stimulation ( $\approx 3$-fold) is achieved at a concentration of $1 \mathrm{mM}$. Stimulation by $1 \mathrm{mM}$ tungstate was completely reversible. Tungstate also activates adenylate cyclase in the brain, heart, lungs, kidneys, and liver of the rat. This suggests that tungstate (sodium) activation of adenylate cyclase is a general phenomenon (Hwang and Ryan 1981).

In rats, dietary $W$ either at 5 or $50 \mathrm{ppm}$ concentrations stimulated the conversion of $\mathbf{L}$-gulonolactone to L-ascorbic acid, $\mathrm{P}<0.02$ and $\mathrm{P}<0.001$, respectively. Tungsten increased the oxidative breakdown of

L-ascorbic acid by rat liver enzymes $(\mathrm{P}<0.05)$. Tungsten at 5 and $50 \mathrm{ppm}$ increased the biosynthesis of L-xylulose from sodium L-gulonate in rat kidney extracts, $P<0.001$ (Chatterjee et al 1973). Rats exposed to a low Mo diet and $100 \mathrm{mg} / \mathrm{kg} \mathrm{W}$ as potassium tungstate in water for 21 days showed significant decreases in total cytochrome P-450 and SO activities of 78 and $30 \%$, respectively, when compared to rats on the control regimen (Chengelis 1991).

No information was located regarding the immunotoxicity of tungsten or tungsten compounds. Several recent acute studies in dogs (Pereira and Grande 1992; Aguas, Grande and Carvalho 1991; Grande et al 1990) and rodents (Peao et al 1993; Peao, Aguas, and Grande 1992) demonstrate that tungsten microparticles or insoluble calcium tungstate powder (a water-insoluble compound) elicit strong inflammatory responses in the lung from macrophages and other cells involved in such responses. One study in mice used both silica and tungsten microparticles and characterized the response according to cell types; the macrophage responses were similar but more prolonged to the tungsten particles (Peao, Aguas, and Grande 1992). Whether extended systemic tungsten exposure would affect the inflammatory response in any way does not appear to have been investigated.

\section{Tentative dose-response relationship}

Although a dose-response relationship appeared to be linear with ovarian tissue exposure to sodium tungstate, techniques do not yet exist to extrapolate this site exposure to a realistic exposure via inhalation or ingestion. Due to data limitations, no best guess could be determined for tungsten's effect on cytochrome P-450 or L-ascorbic acid metabolism. Because the effects of tungsten given in vitro cannot, at this time, be related to tungsten exposure via inhalation or ingestion exposure in humans, study of these effects is not recommended at this time under the auspices of this project. 


\section{Research recommendations}

It appears that no systematic evaluation of potential immunotoxicity is likely to have been performed, particularly for chronic low-dose systemic tungsten exposure. This is an area for which at least preliminary studies, perhaps subacute high-dose experiments, should be considered.

\section{F.3 CONCLUSIONS}

The review of the literature on tungsten effects suggests that chronic low-dose exposure by environmentally relevant routes (primarily ingestion, but also inhalation) may result in adverse effects on three sensitive and important endpoints. These include neural function, female and possibly also male reproductive function, and teratogenicity or developmental toxicity. The animal data are sparse and in all cases rest in large part on studies reported 25 to 30 years ago by one Russian laboratory. Yet enough other work has been done to indicate strongly that further investigation is warranted to replicate or substantiate these findings. The data are not strong enough at present to warrant decisionmaking based on the information at hand.

The evidence for growth effects and for effects from inhibition of various enzymes or enzyme systems, SO and XO in particular, suggests that adverse health effects for humans are not apt to be of significant concern from low-dose long-term tungsten exposure. Extreme dietary molybdenum deficiency would have to be coupled with the tungsten exposure to have effects on XO and SO of any concern except for individuals congenitally deficient in SO for whom the diet would also have to be high in sulfite in order for adverse effects to be seen. These abnormal dietary conditions seem unlikely in the extreme.

\section{F.4 REFERENCES}

Aamodt, R. L. 1975. Retention and Excretion of Injected ${ }^{181} \mathrm{~W}$ Labeled Sodium Tungstate by Beagles. Health Physics 24:519-524.

Aguas, A. P., N. R. Grande, and E. Carvalho 1991. "Inflammatory Macrophages in the Dog Contain High Amounts of Intravesicular Ferritin and Are Associated with Pouches of Connective Tissue Fibers," Am. J. Anat. 190, 89-96.

Grande, N.R. et al. 1990. "Time Course and Distribution of Tungsten-Laden Macrophages in the Hilar Lymph Nodes of the Dog Lung after Experimental Instillation of Calcium Tungstate into the Left Apical Bronchus," Lymphology 23, 171-182.

Lison, D. and R. Lauwerys 1990. "In Vitro Cytotoxic Effects of Cobalt-Containing Dusts on Mouse Peritoneal and Rat Alveolar Macrophages," Environ. Res. 52, 187-198.

Lison, D. and R. Lauwerys 1991. "Biological Responses of Isolated Macrophages to Cobalt Metal and Tungsten Carbide-Cobalt Powders," Pharmacol. Toxicol. 69, 282-285.

Lison, D. and R. Lauwerys 1992. "Study of the Mechanism Responsible for the Elective Toxicity of Tungsten Carbide-Cobalt Powder Toward Macrophages," Toxicol. Lett. 60, 203-210. 
Lison, D. and R. Lauwerys 1993. "Evaluation of the Role of Reactive Oxygen Species in the Interactive Toxicity of Carbide-Cobalt Mixtures on Macrophages in Culture," Arch. Toxicol. 67, $347-351$.

Lison, D. and R. Lauwerys 1994. "Cobalt Bioavailability from Hard Metal Particles. Further Evidence that Cobalt Alone Is Not Responsible for the Toxicity of Hard Metal Particles," Arch. Toxicol. 68, 528-531.

Peao, M. N., A. P. Aguas, and N. R. Grande 1992. "Cellular Kinetics of Inflammation in the Pleural Space of Mice in Response to the Injection of Exogenous Particles," Exp. Lung Res. 18, 863-876.

Peao, M. N. et al. 1993. "Inflammatory Response of the Lung to Tungsten Particles: An Experimental Study in Mice Submitted to Intratracheal Instillation of a Calcium Tungstate Powder," Lung 171, 187-201.

Pereira, A. S. and N. R. Grande 1992. "Particle Clearance from the Canine Pleural Space into Thoracic Lymph Nodes: An Experimental Study," Lympphology 25, 120-128. 



\begin{abstract}
APPENDIX G
METHODS FOR ESTIMATING CANCER POTENCY OF TUNGSTEN IN HUMANS
\end{abstract}

T. D. Jones and C. E. Easterly 



\section{APPENDIX G. METHODS FOR ESTIMATING CANCER POTENCY OF TUNGSTEN IN HUMANS}

\section{G.1. INTRODUCTION}

Roughly $10,000,000$ chemical compounds are involved in commercial processes as ingredients, end products, or waste by-products and about 65,000 are in common usage. From this inventory, only about two dozen chemical compounds have been identified as known human carcinogens by epidemiological studies. A "carcinogen" (classified from epidemiological results) may be defined from positive exposure-response correlations. Thus, although statistical analyses have been used to model the isolated effects of the particular hazard of concern, the "human carcinogen" always carries possible contamination forces from an array of environmental and endogenous carcinogenic initiators and promoters. While the collection of all such factors appears to be subcarcinogenic (i.e., causes the same cancer rates as found in suitable control populations), it is possible that the cofactors may amplify or actually complete the carcinogenic action of the hazard of interest. Typically, occupational exposures are complex in numbers of compounds, concentrations, and times so that often technologies or processes are specified as carcinogenic without identification of the specific roles of the individual insults that comprise the total exposure burden.

A "carcinogen" (classified from laboratory results) is expected to cause cancer when amplifying factors internal to or arising from the biological test model are minimized. More than 500 compounds have tested as positive in animal carcinogenic models. The number of carcinogens identified, either human or animal, is unrealistically low because of the rigor, time, and expense mandated by testing of the null hypothesis, i.e., a compound/exposure is not carcinogenic until epidemiological and/or experimental evidence indicates otherwise at the $95 \%$ level of significance.

Within the real-life complex exposure scenarios for humans, it should be recognized clearly that subtoxic and sub-carcinogenic interactions with low, background doses of carcinogens are distinct realities based on the studies of Ames regarding the concentrations of natural carcinogens found in normal human foods (Ames 1990). Probably fewer than 1/1000 compounds of potential concern have been studied to date either by epidemiological methods or in animal tumor models. Literally thousands of compounds are of great concern to human health and the historical approach has been to test them one by one in the two-year chronic rodent bioassays as used formerly by the National Cancer Institute (NCI) studies and currently by the National Toxicology Program (NTP) experiments. Each compound tested in this fashion usually requires a span of five or more years through its initial testing, planning, two-year dosing period for mice and rats, plus analysis, reporting, and peer review phases. Typically, about 4 million dollars are required for each compound so tested and only about 10 new compounds can be considered per year. Even after all of this effort, a significant number of the compounds tested either produce dose-response results below the level of statistical significance for the number of animals used, or give ambiguous results in the different sexes and/or species (sometimes strains) of genetically uniform rats and/or mice as illustrated in Fig. G.1. As shown in Fig. G.1, the correlation between $\mathrm{TD}_{50}$ (the dose that causes tumors in 50\% of the test animals) in rats and mice (Gold et al. $1989)$ is reasonably good for 33 compounds $(r=0.81)$ if test results in both species are positive (Jones, 1996). Unfortunately, there were another 22 compounds reviewed by Jones (1996) that gave positive results in one species and negative results in the other (Gold et al. 1989). Thus, predicting carcinogenic potency is an imprecise process and it is likely that rodent-to-man comparisons are more uncertain than rodent-to-rodent comparisons. It may be discovered that mechanism-of-action based 


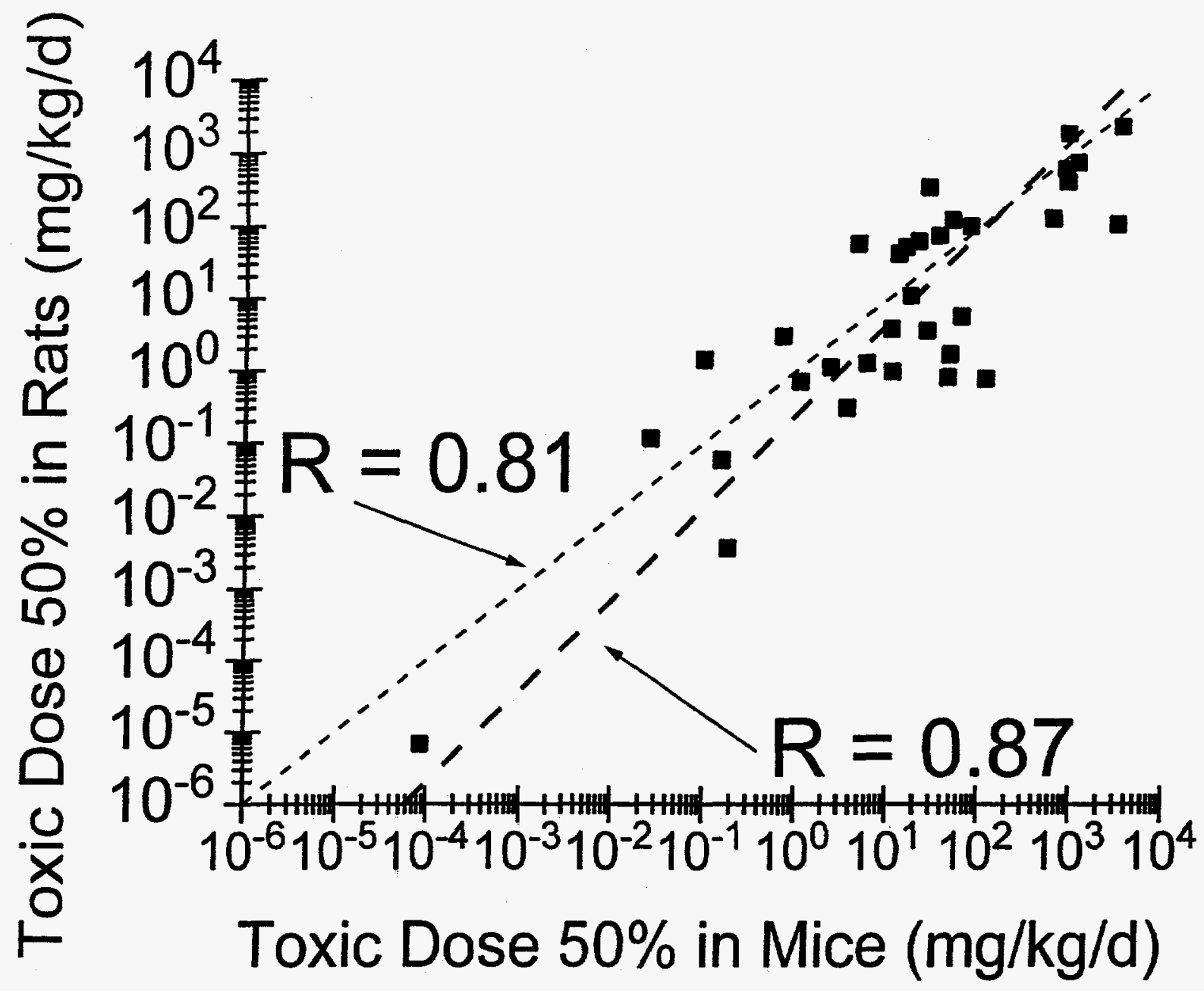

Fig. G.1. Comparison of the $\mathbf{T D}_{50}$ (i.e., the dose that reduces the number of tumor-free animals by $50 \%$ ) in mice vs the $\mathbf{T D}_{50}$ in rats for the 33 compounds in the list of 101 for which positive test results were obtained in both mice and rats. The correlation coefficient was 0.81 , but there were 22 other compounds that tested positive in one species and negative in the other.

models provide the only reliable basis for risk assessment, but attempts to date have not demonstrated much improvement over simple hazard ranking considerations (Hileman 1993). Traditionally, "equivocal" compounds are retested with modified experimental protocols and frequently the second period of testing does not reduce the uncertainty deriving from the original study.

Within the basic research community, practically all of the scientific enthusiasm and available fiscal resources are consumed in the chase of the molecular mechanisms of tumorigenesis. However, such focal efforts may continue to fail because the pathological endpoint known as "neoplasia," "cancer" or "tumor" results from an almost uncountable number of molecular, gene, chromosome, mitochondrial, membrane, cellular, organ, humoral, and chemical processes. Although many molecular mechanisms of toxicity are known, others continue to be discovered. Mechanisms that hold for one particular compound or class of compounds for a particular dosing protocol may have little, if any, relevance for other carcinogenic insults and/or other dosing protocols of the same substance. 
Although the cells of most cancers are monoclonal in origin, a rather large bloom from a single parent cell or an infusion of many such cells seems to be required to express a cancer in a test animal. Few experiments have ever successfully produced a cancer in test animals with a graft or transfusion insult bolus of fewer than 10,000 such cells per animal-even in an immunosuppressed host animal such as an irradiated, a thymic (nude) mouse. Certain protocols involving chemical or mechanical potentiators of carcinogenesis or periods of natural organogenesis (e.g., embryogenesis or puberty) or hyperplasia as a result of toxic injury may provide exceptions. In the worst case, cancer can be hypothesized to result from a single cell, as implied by the somatic mutation theory, and a few novel experimental designs have indeed produced cancers or leukemias from infusions of only one or two cells per host animal. Based on normal intermitotic cycle time for hematopoietic stem cells, it appears that clinical leukemia might be able to bloom from a single parent cell in one or two years-which is quite consistent with the shortest latency periods observed in the Japanese atomic bomb survivors.

What is actually needed is a classification or ranking system based on toxicity to biological molecules at meaningful dose levels. Thus, great enthusiasm has been devoted to analyzing various structureactivity relationships (SAR) in attempts to develop a predictive index for the carcinogenic potential of various chemicals. This is far more complex, but in a way analogous to the periodic chart based on orbital electrons. To date, the SAR-based models have not achieved sufficient predictive accuracy to be very useful across chemical classes and major inconsistencies may result for complex biological molecules.

With regard to reactivity of carcinogenic compounds and/or substances that promote or potentiate carcinogenic lesions that have been seeded by other insults, many techniques have been tested but to date no system based on first principles from chemistry or physics has alleviated the problems of chemical dosimetry for carcinogenic risk. A common-scale approach would use test results from a spectrum of bioassays ranging from bacteria to carcinogenesis testing in mammals in order to empirically map some aspects of the hypothetical potency/reactivity chart for molecular toxicity to humans. This approach is considered as the ultimate dose-response detector in terms of cost and accuracy.

The objectives of this study include establishing direct or common-scale comparisons between (a) the radiologically-based risks from exposure to depleted uranium with (b) the toxicologically based risks associated with exposures to tungsten and tungsten-related compounds. Useful comparisons should take into account the numerous aspects of: (1) exposure source terms, (2) population and personal exposure pathways, (3) solubility, absorption, penetration into the human body, (4) target-organ specific doses, (5) differential toxicity equivalent factors as measured in different but relevant biological test models, (6) hazard or risk of the source-specific spectrum of untoward health effects as a consequence of exposure, and (7) uncertainty aspects of both radiological and chemical insults.

Earlier appendices have focused on some of these aspects. This Appendix will concentrate on items (5) differential toxicity equivalent factors as measured in different but relevant biological test models and (7) uncertainty aspects of both radiological and chemical insults-restricted to the differential toxicity equivalent factors. 


\section{G.2 HISTORICAL CONCEPTS AND APPROACHES TO CHEMICAL VS RADIOLOGICAL RISK}

\section{G.2.1 Risk/Hazard Assessments}

Most risk assessments have avoided any common-scale approach to comparing of risk for chemical and radiological hazards. ${ }^{1}$ Meaningful, direct comparisons should include realistic (i.e., accurate) comparisons of both the expected frequency of disease and the severity of the disease endpoints, but comparisons based on both qualitative (severity-specific) and quantitative (rate-specific) models are usually not achievable from meager resources typically devoted to assessments of risk. Also, important disease-amplifying exposures and health endpoints associated with immunotoxicity, neurotoxicity, reproductive effects, and cardiovascular diseases such as atherosclerosis should be included. Unfortunately, time and resources rarely permit any health effects study to treat these concerns in reasonable depth, but those considerations should be addressed realistically before policy decisions can be made in confidence that a hazard from one option is comparable to, or smaller, than the hazard from an alternative option, especially when the policy decisions affect immense monetary budgets, human health, and environmental quality on a global scale.

In general, toxicity can be conceptually associated with chemical reactivity. ${ }^{2}$ For atoms, the chemical periodic chart may provide some limited perspective of chemical reactivity that may be linked by ratio and proportion to toxicity data for other atomic substances in the absence of relevant test data for the substance of interest. Atoms in the same vertical group such as Group IV-B or VI-B have the same number of electrons in the outer shell and may be more relevant than a substance from the same row within the transition metals where the same electronic configuration occurs, except for the number of

\footnotetext{
${ }^{1}$ One exception is the common-scale risk assessment approach for "Protection of Human Health from Mixtures of Radionuclides and Chemicals in Drinking Water" by Jones, Owen, and Trabalka (1991) who defined two such common-scale index points-one based on safety and the other based on risk. The safety-based index was linked to common population exposures resulting from food and water intake of compounds generally regarded as safe, i.e., the GRAS concept as promulgated by the Food and Drug Administration (FDA 1985). The risk-based common-scale bridge attempted to provide parallel treatments of similar precision between chemical and radiological carcinogens by eliminating the inflationary effects of models and assumptions, i.e., the goal was to eliminate subjective, variable margins for safety.

${ }^{2}$ Paracelsus (1493-1541) proposed the basic operational blueprint of toxicology-“all substances are poisons; there is none which is not a poison, the right dose differentiates a poison and a remedy" (Klaassen et al. 1986). Cancer is the end result of toxic injury and evidence is abundant that "carcinogen" can probably be substituted for "poison" in Paracelsus' synopsis provided that the classical impression of dose involving milligrams of test agent applied per kilogram of body weight treated can be extended to include variations in: (1) route of administration, (2) rate of application, (3) duration of treatment, and (4) tuning the dose, route, rate, and duration variables in combination. Consistent with Paracelsus' thesis, Ecobichon (1992) noted that
}

Toxicology may be defined as 'the qualitative and especially the quantitative study of the injurious effects of chemical and physical agents as observed in alterations of structure and response in living systems. . .'. . . The descriptive term 'qualitative' in this definition pertains to whether or not a particular agent is toxic and to the concept of relative toxicity of one agent in comparison with the known toxicity of other agents.

A basic tenet of toxicology is that the more frequently a specific adverse health effect can be measured in different animal species, the greater is the likelihood that, at some dosage, a similar effect might occur in the human. 
electrons in the outer shell. When toxicological test data for the element of interest are inadequate, then comparisons with other groups and rows of the Periodic Table may be used, but conclusions are weakened according to the relative positions shown in Fig. G.2. For example, molybdenum might be the best surrogate for tungsten but if inadequate bioassay data are available for molybdenum, then alternatively, chromium might be the most informative. Somewhat less ideal would be data from the same row and the greater the reach, the greater the uncertainty.

Transition metals

\begin{tabular}{ccccccccccc}
\hline Group III B & IV B & V B & VI B & VII B & \multicolumn{2}{c}{ <Group VIIl } & I B & II B \\
\hline Sc & Ti & V & Cr & Mn & Fe & Co & Ni & Cu & Zn \\
Y & Zr & Nb & Mo & Tc & Ru & Rh & Pd & Ag & Cd \\
La/15 & Hf & Ta & W & Re & Os & Ir & Pt & Au & Hg \\
\hline
\end{tabular}

Fig. G.2. Comparison of position in the periodic chart for transition metals that may be used as a reference for the toxicity of tungsten.

Relative assessments based on the periodic chart should be used only when bioassay data are not available for the substance of interest or a close homologue. It would seem that toxicity data for metallic compounds should be readily available, but in general, metals have been tested very rarely compared with organic compounds. Perhaps three reasons explain this paucity of data: (1) the generally-perceived chemical inertness of many inorganics, (2) lack of concern about any significant degree of biotoxicity, and (3) the difficulties associated with experimental design and conclusions deriving from testing an insoluble substance in biological models. 


\section{G.2.2 Common Scale for Mutagenic Hazards}

Committee 17 of the Environmental Mutagen Society (1975) proposed two similarly-conceived benchmark standards for environmental screening of mutagenic chemical insults. ${ }^{3}$ The first, the ratedoubling concentration, was to be based on the spontaneous mutation rate; the second, the remequivalent chemical (REC), was defined as that dose of a chemical which produced the same amount of genetic damage as 1 rem of chronic radiation exposure. They also made the point that doseresponse curves would be a far stronger basis for extrapolation to humans than single-dose tests. At this time a freeze on nuclear power production, and a de-emphasis on other nuclear industries, lead to lack of funding for the development of scientific background documents and impact statements that would have provided the basis for direct comparisons between radiobiological effects associated with nuclear applications and toxicological effects associated with chemical exposures. However, the operational logic behind Committee 17's efforts is valid today and can be applied to other disease endpoints such as cancer, immunotoxicity, neurological effects, and cardiovascular diseases that may

${ }^{3}$ Members of Committee 17 included: John Drake, Seymour Abrahamson, James Crow, Alexander Hollander, Seymour Lederberg, Marvin Legator, James Neel, Margery Shaw, Eldon Sutton, R.C. von Borstel, and Stanley Zimmering. Fredrick de Serres and Gary Flamm served as consultants to Committee 17. The quality of their effort was consistent with the imposing credentials of the contributors.

Abstractions of their own text as published in Science most effectively describes the goal of Committee 17:

Mutations can arise in both somatic and germ line cells. Somatic mutations, and its probably close correlate, carcinogenesis are already recognized as immediate toxicological problems. . . It will be important when extrapolating mutation rates from test systems to man to employ certain basic units. Two units are suggested for adoption at this time, one based upon the spontaneous mutation rate and one upon radiation equivalents. The suggested units are: 1) The rate doubling concentration (sic., doubling of the natural spontaneous rate) . . 2) The rem-equivalent-chemical (REC)... The REC is that dose or product of concentration multiplied by time which produces an amount of genetic damage equal to that produced by 1 rem of chronic irradiation. . Quantitative extrapolation to man should be based not simply upon single-dose tests, but instead upon dose-response curves.

In conclusion, Committee 17 noted that

Human populations are now exposed to a wide variety of compounds never before encountered in the history of man. Many of these compounds are clearly mutagenic to lower organisms, and there are sound biological reasons to conclude that at least some are also mutagenic to man. Since the vast majority of detectable mutations are deleterious, an artificially increased human mutation rate would be expected to be harmful in proportion to the increase.

A number of test systems are now being developed and perfected to the point where they can detect chemical mutagenesis reliably and sensitively. No system by itself meets all the requirements of an ideal system, but the available systems collectively provide important information about the mutagenic potential of many, perhaps most chemical compounds.

When data from the best available mutagenicity screening systems are combined with information about the distribution of compounds in the environment and their metabolic fates in the body, it becomes possible to make at least preliminary estimates of the health hazards posed by environmental mutagens. It is therefore already reasonable to begin to regulate the distribution of chemical compounds.... 
develop in human populations exposed over a long period of time to any substance-even one that is seemingly "inert."

\section{G.2.3 Progression of Carcinogenesis}

\section{G.2.3.1 Holistic Approach}

In 1980 Kouri, Henry and Kreisher of MicroBiological Associates produced a schematic diagram that integrated the various stages, forces, and cofactors of carcinogenesis into a holistic blueprint. That effort, reproduced in Fig. G.3 with permission, places various aspects of initiation, biochemical promotion, cellular promotion, cocarcinogenesis, route of administration, nutrition, etc. into the pathway defined by the etiological movement from a single genotypically and phenotypically normal cell into a phenotypically aberrant cell. If the phenotypically altered cell is contained within a tissue matrix that has high mitotic rates, then when the size of the aberrant clone becomes sufficiently large (e.g., some experiments suggest 10,000 cells both for liver and for the hematopoietic bone marrow), the cell-to-cell homeostatic control (i.e., includes both the contact inhibition and humoral chalones) mechanisms are inadequate to keep the neoplastic focus from acquiring autonomous growth characteristics. $^{5}$

From the Kouri model, one can conclude that initiation is a binary process and that initiation alone is likely to remain subcarcinogenic and subclinical throughout an entire lifespan in all but the most rapidly renewing cells of the body (possibly including spermatogonia, lymph glands, crypt cells of the gut, and hematopoietic stem cells of the marrow). Cell proliferation, (which the Kouri model links deterministically to carcinogenic outcome) is consistent with: (a) the general biological theory proposed by Trosko and Chang (1980) involving monoclonal development of mutagenic lesions into cancer, diabetes, and atherosclerosis and (b) the biologically-based mathematical models of relative potency, cytotoxicity, cell proliferation, and risk as developed by Jones et al. $(1979,1983,1984)$.

\footnotetext{
${ }^{4}$ Consistent with ideas of Paracelsus, the observations of Bergonnie and Tribondeau (1906) led to a classical description that a tissue is more radiosensitive the more undifferentiated its cells are morphologically and physiologically, the more active they are mitotically, and the longer they remain in an active state of proliferation (the more divisions they undergo between the youngest precursor cell and the mature functional cell). These generalizations can be applied to toxicology as readily as they have been used in radiobiology.

${ }^{5} \mathrm{~A}$ second advantage of the Kouri et al. integration is that bioassays can be placed within the big picture of cancer genesis without distortion resulting from models driven only by DNA-related events; i.e., non-DNA processes, especially those involving membranes and production of cytokines, are important to carcinogenic risks, especially for insults that are not directly genotoxic. The considerations of Committee 17 address carcinogenic initiation within the Kouri model, but the bridge between oncogenic initiation of pre-carcinogenic cell(s) and the clinical cancer endpoint involves realistic models of compensatory cell proliferation as a rate-limiting modulator.
} 


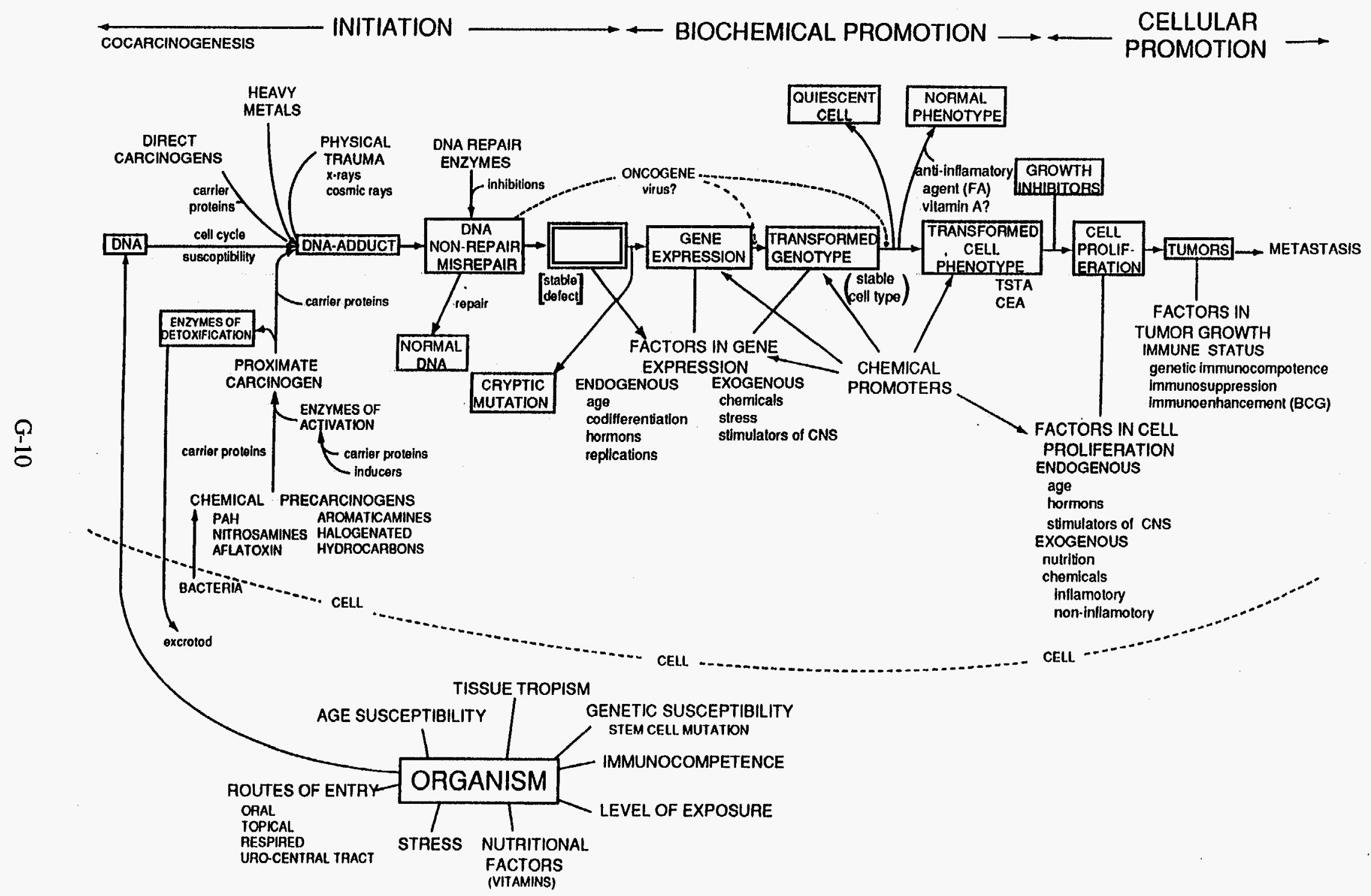

Fig. G.3. Stages in carcinogenesis as described by Kouri, Henry, and Kreisher (1980). 
Thus, the Kouri model provides a unified, realistic analytical structure with which to improve assessments of risk. ${ }^{6}$

Currently EPA has shown a tendency to use changes in biochemical rates, enzyme levels, or molecular binding as indicators of low-dose toxicity. As an alternative, one may adopt a perspective as described by Conolly (1994) regarding the EPA's reassessment of 2,3,7,8-TCDD, viz., "A toxic chemical is defined as '. . any agent that is capable of producing injury or death when ingested or absorbed' . . A toxic effect is also, by definition, an adverse health effect. . . induction of biochemical events is a universal characteristic of all xenobiotics and is not equivalent to toxicity." Available data on human leukocyte counts suggests that as much as $30 \%$ variation can occur from day to day without any known toxic exposures. In addition to the questions relating to the meaning of such spontaneous rate changes, the question arises as to what the appropriate normal baseline or control values are for individuals and populations for measurements associated with low concentrations of chemicals. In fact, most chemical exposures, even sub-carcinogenic doses of known carcinogens and chemical noncarcinogens may be associated with biochemical rate changes. If non-carcinogenic compounds can be associated with those same rate changes, then they should not be taken as either quantitative or qualitative measures of carcinogenic risk.

Figure G.4, based on traditional toxicological indicators of dose response endpoints such as death, cytotoxicity, No-obvious-adverse-effects levels (noaels) and lowest-obvious-adverse-effects levels (loaels), illustrates that biochemical rate changes or fluctuations in cell populations should not be taken as positive indications of carcinogenic risk at low doses. Thus, the methods proposed for assessments of comparative risks from depleted uranium- and tungsten-related compounds are based on biological

\footnotetext{
${ }^{6}$ It appears that all of these efforts-even those by Committee 17 and the Kouri team-preceded demand at the scientific market-place because no such concept received much interest for nearly a decade. Nevertheless, researchers continued to develop theories, experiments, and models and by the late 1980's the mechanistic importance of toxicity-induced compensatory cell proliferation became fundamental to recommendations promulgated by the American Conference of Governmental Industrial Hygienists, the National Council on Radiation Protection and Measurements, and the International Agency for Research on Cancer. That acceptance seemed opportunistic in the sense that most individuals associated with those activities had repeatedly rejected etiological models of carcinogenesis based on compensatory cell proliferation, preferring instead models based on somatic mutation theory whereby one molecule can, in principle, change one cell and that one cell may, in theory, mutate and, in and of its own forces, complete a frank cancer. The change may have been influenced in the background by views and reviews indicating that historical experimental studies had been either incorrectly designed or misinterpreted with regard to claims that compensatory proliferation and tumor promotion were mechanistically distinct. Most of the pre-1980 experiments interpreted as demonstrating that toxic hyperplasia and tumor promotion were distinct, disjoint processes can be explained by the observation that toxic hyperplasia was usually evaluated from a single dosing. Now, it is well known that biological tolerance can be developed for many compounds after an initial toxic response. One barrier associated with proliferation-based models was removed when experimental studies demonstrated that a simple abrasion wheel or even repeated tape stripping of hair follicles could be used to produce identical histological effects and an equivalent neoplastic yield as obtained from a chemical extract of croton oil (TPA). Croton oil had been viewed as having unique chemical and inorganic properties producing proliferative changes that were distinct from cell proliferation caused by mechanical insults and hyperplastic doses of so-called "non-carcinogenic" chemical compounds; many experiments have reported that belief. By the 1990s, research strategies used to study molecular toxicology at the Chemical Industry Institute of Toxicology (1989) were focused on cell proliferation and continue to help understand the complex molecular mechanisms that underlie toxic hyperplasia and the carcinogenic process.
} 


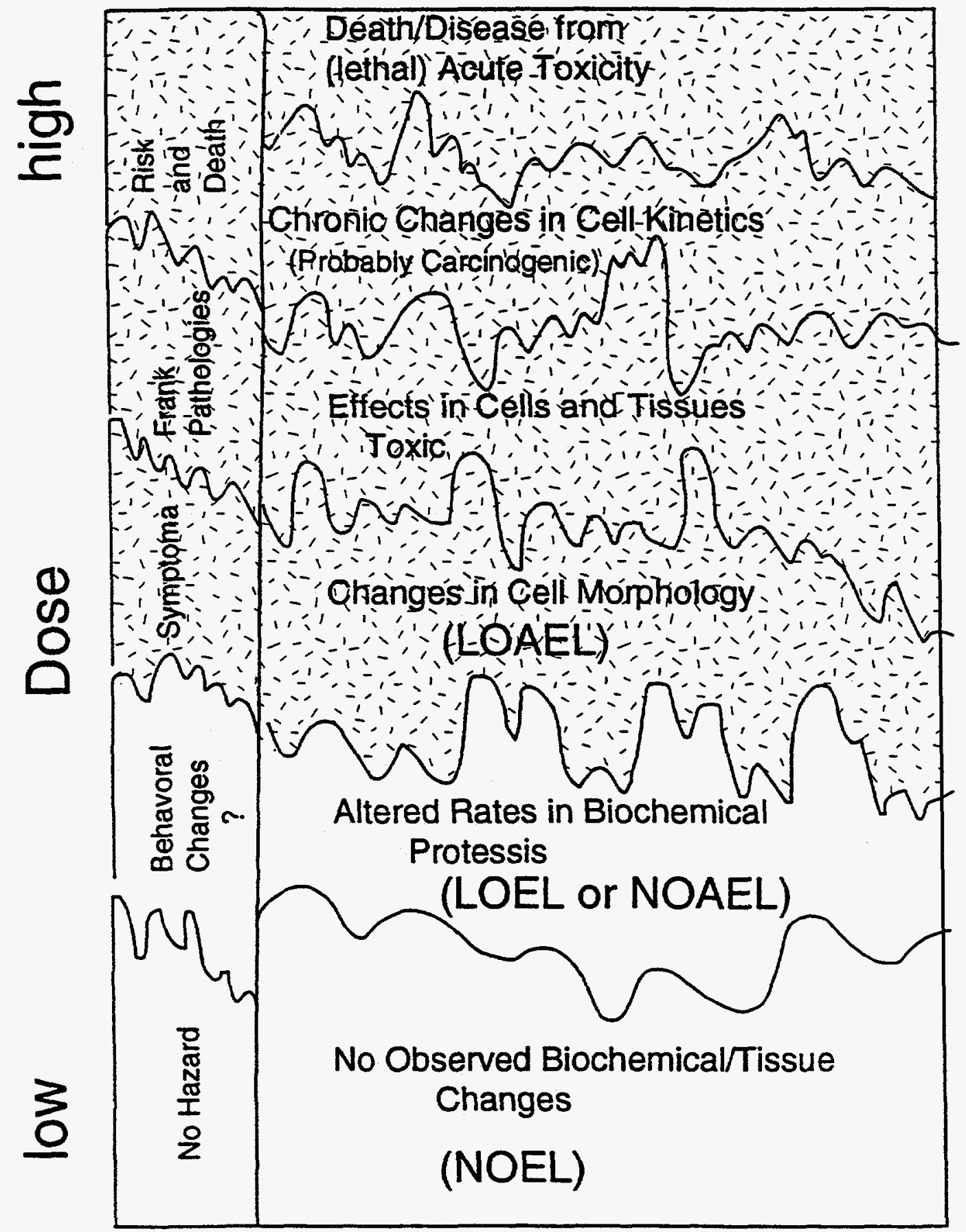

Fig. G.4. Conceptualized schematic to illustrate the subjective boundaries within the toxicological continuum. The comparisons between tungsten and depleted uranium will avoid regions associated with LOEL, NOAEL, and NOEL in contrast to the EPA's analysis of 2,3,7,8-TCDD. 
and/or clinical observations of toxicity, not upon observations of biochemical fluctuations or variability in cell counts against a normally changing background.

\section{G.2.3.2 Recent Conceptual Support for the Role of Proliferation in Carcinogenesis}

Recommendations by the ACGIH: By 1985, the American Conference of Governmental Industrial Hygienists had recommended that "There is increasing evidence that physical irritation may initiate, promote or accelerate physical impairment through interactions with other chemical or biological agents."

Comparative Carcinogenicity as Evaluated by the NCRP: In a review of "Comparative Carcinogenicity of Ionizing Radiation and Chemicals," the National Council on Radiation Protection and Measurements (NCRP 1989), noted that initiation of carcinogenic cells is irreversible and immediate after dosing either with chemicals or radiation and that most carcinogens are mutagens. Furthermore, DNA synthesis and subsequent mitosis stabilizes the lesions of initiation so that promotion can express these events as frank neoplasms at any later time. The NCRP review also remarks that "promoters appear to be tissue-specific but have in common the property that they induce proliferation in the target tissue."

Although NCRP Report 96 provides a fairly comprehensive summary of macroscopic carcinogenic processes associated both with radiation-induced and chemical carcinogenesis, the NCRP effort fails to provide a useful common-scale unit of either risk or dose so that direct comparison can be made. The NCRP report does provide some relative potency comparisons between chemical compounds but that exercise has been attempted by numerous investigators and dates to the well-known Iball Index (1939). In conclusion, the NCRP noted that: carcinogenic effects by certain chemicals are similar to ionizing radiation; cancers by both types of agents are individually indistinguishable from those by other causes; in many cases, risks can be estimated only by modeling that involves interpolation and/or extrapolation.

Although NCRP Report 96 recognized cell proliferation as being intrinsic to carcinogenesis, the committee that wrote NCRP Report 96 became hypersensitive to differences between carcinogenic agents (i.e., microscopic details) and stopped far short of a practical approach (i.e., concept) like doubling dose, GRAS exposures, or the REC as proposed by Committee 17 of the Environmental Mutagen Society (1975). Overall, the NCRP report was a major disappointment because their reasons for not providing a common scale of dose/risk could just as logically be attached to ionizing radiations of different quality as estimated from linear energy transfer or stopping power values. Yet because of historical acceptance of an RBE (Relative Biological Effectiveness) factor, radiation protection groups have grown comfortable when such relative potency comparisons are restricted to radiation biology as seen in NCRP Publication 104 (1990). Cellular processes involving sublethal injury, repair, and multistep killing are extremely distinct for radiations of different quality. Similarly, the frequencies of particular types of DNA base alteration, single strand breaks, double strand breaks, and other lesions including damage to membranes and organelles as produced by low-vs high-LET radiations are such that the NCRP would no longer be able to accept an RBE concept given the same reservations as described for rejecting a numerical bridge from radiobiology to chemical toxicology.

IARC on Cell Proliferation: In IARC Scientific Publication 116, the International Agency for Research on Cancer wrote (1992): 
Cell proliferation has been linked experimentally or conceptually to the carcinogenic process and is an important mechanistic aspect for both genotoxic and nongenotoxic carcinogens . . . Cell proliferation may act at each stage of the carcinogenic process, altering the size of the pool of cells at risk for the next event. The pool sizes are dependent on the kinetics of cell birth, cell death, and cell differentiation.

Thus, by the 1990 s the general scientific community came to realize the significance of Bergonnie and Tribondeau's observations of 1906, but individuals and groups still remain uncertain about the simple relative potency comparisons of John Iball and subsequent efforts to compare relative reactive/toxicological potentials of an array of compounds, especially those that did not limit comparisons to a particular chemical class such as PNAs. If the simple non-source-specific horsepower rating of an (a) animal/machine/chemical/gravitational capacity to perform work is taken as an analogy for the potency of various compounds, then clearly the underlying mechanisms do not enter into the logic of force (concentration) applied to achieve a measure of work (response).

From this perspective, it is not necessary to know the mechanisms involved in order to estimate carcinogenic risk associated with a chemical. Because of the correspondences between initiation, cytotoxicity, inflammation, cell proliferation, and neoplasia it seems that effects on compensatory cell proliferation may serve as a unifying index. If this approach results in error, it would result in erring on the side of safety for exposed human populations by considering not only initiators but also nongenotoxic chemicals affecting later stages of the carcinogenic progression.

The evidence, some of which is summarized above, is compelling that compensatory cell proliferation has been found to be essential for preserving initiation of precarcinogenic molecular lesions (by blocking repair of sublethal DNA lesions or cellular injury) and for causing a bloom from a single, mutated or transformed cell into a frank cancer.

\section{G.3 CARCINOGENIC HAZARDS FROM CHEMICALLY AND BIOLOGICALLY "INERT" COMPOUNDS}

Throughout the history of carcinogenic testing, the pervasive attitude has been that only very reactive compounds are logically carcinogenic-and conversely any inert or less reactive substance is of marginal, if any, concern. However, occupational histories have demonstrated clearly and repeatedly that even inert, non-reactive compounds can be associated with chronic irritation, fibrosis, impairments such as hyperplasia, metaplasia, asbestosis, silicosis, hard-metal disease, white-lung, black-lung, and carcinogenesis. Clearly, a toxic exposure, even to inert substances, produces a spectrum of toxic endpoints consistent with Figs. G.3 and G.4. Toxicology viewed as a continuum seems to dovetail closely with risk factors associated with occupational carcinogenesis. Furthermore, non-essential metals such as zirconium and tungsten may interact with essential trace substances to change the normal concentration of the essential trace metal available to organ tissue (Stokinger in Patty's Industrial Hygiene). As two examples of relevance, the NTP is beginning a two-year rodent study on molybdenum trioxide and vanadium pentoxide (Note: molybdenum is between chromium and tungsten in class VI-B of the periodic chart while vanadium is chromium's class V-B counterpart (Fig. G.2). Also, it should not be overlooked that the Gold (1984) carcinogenic potency data base contains NCI-NTP test results for sodium tungstate, vanadyl sulfate, and zirconium (IV) sulfate.) Although some results are "not statistically significant," there was adequate concern that caused the long-term 
rodent testing to be done and concern for human safety would mandate that chronic exposures to these compounds be considered as potentially hazardous unless convincing epidemiological data indicate otherwise.

Gunnison et al. (1981) studied the subchronic toxicology in normal rats and mice made sulphiteoxidase deficient by administration of tungstate. "The sulphite-oxidase-deficient rats were outwardly as healthy as controls and exhibited normal weight gain and maintenance over the 9-week test period. A 4/149 incidence of mammary adenocarcinoma was observed in sulphite-oxidase-deficient rats, all in rats of age less than 5 months, compared to 0/143 observed in age-matched rats with normal sulphiteoxidase. Although this result was not statistically significant, the rarity of spontaneous tumors of this type among rats of this age suggests that these carcinomas may, in fact, have been treatment related."

Table G.1 summarizes the behavior of 18 "inert" substances in various biological test models. Only a few years ago, all with the possible exception of asbestos would have been treated as noncarcinogenic. Only a few decades ago, asbestos was used freely as a non-carcinogen. In fact, only a dozen years ago, so-called solid-state carcinogenesis, except for asbestos-induced mesothelioma, was considered a laboratory curiosity and of no particular relevance to humans, except for implanted devices. The discussion here focuses on metallic compounds and inert substances, but similar effects can be cited for organic compounds, the best known of which is perhaps benzene. Only a few years ago, benzene was considered to be non-carcinogenic and was in fact used as a carrier to test other compounds of carcinogenic concern.

Table G.1. Summary of effects data from substances usually considered biologically inert

\begin{tabular}{lcccc}
\hline & Mutagenic to cells & $\begin{array}{c}\text { Tumorigenic to } \\
\text { animals }\end{array}$ & $\begin{array}{c}\text { Tumorigenic to } \\
\text { implant }\end{array}$ & $\begin{array}{c}\text { Carcinogenic to } \\
\text { man }\end{array}$ \\
\hline $\begin{array}{l}\text { Asbestos } \\
\text { Calcium }\end{array}$ & Yes & Yes & Yes & Yes \\
Coal dust & Yes & Yes & Yes \\
Copper & Yes & Yes & & \\
Copper sulfate & Yes & Yes & & \\
Fiberglass & & & Yes \\
Gold & Yes & & & \\
Graphite & & Yes & Yes & \\
Iron (and ore) & & & \\
Platnium & & Yes & \\
Quartz/silica & & Yes & \\
Sand & & Yes & \\
Silver & Fumes & Yes & & \\
Stainless steel & (Fes) & & Yes \\
Talc & & & \\
Tin & & & \\
Teflon & & & & \\
\hline
\end{tabular}


Some of the modern composites, whiskers, and fibers have been tested more thoroughly than metallic compounds having a long history of usage. For example, bioassay results for testing/screening of hafnium-, tungsten-, and zirconium-related compounds are summarized in Table G.2. Data in Table G.2 are taken from the Registry of Toxic Effects of Chemical Substances (RTECS). Based on policy driven by the null hypothesis, there would seem to be little cause for limiting human exposures to most of these compounds. However, the absence of positive test results for a compound does not mean that it is non-carcinogenic or even non-toxic. In fact, the simple observation that some compounds listed in Table G.2 have been found to cause some positive test results in mutagenic,reproductive, and tumorigenic tests mandates that the potential hazard should be quantified if large populations are exposed.

\section{G.4 CARCINOGENIC TESTING}

As described earlier, long delays, great expense, and much uncertainty are inherent in carcinogenesis testing - even that associated with the rigorous NTP two-year dosing schedules used for mice and rats. That degree of testing is inappropriate for hazard screening and may not do the job as well as simple hazard screening based on a large battery of much less costly bacterial and mammalian cell bioassays. For example, the International Programme on Chemical Safety (1990) evaluated various short-term tests as components of screening for carcinogenic potential and confirmed the value of a battery of tests for various aspects of genotoxicity such as gene mutation, chromosome aberration, unscheduled DNA synthesis, and micronucleus induction. The lack of predictive capability of these tests for nongenotoxic carcinogens acting presumably at later stages of the carcinogenic progression was also acknowledged. ${ }^{3}$ However, no consensus has since been reached regarding the specific tests that should make up a standard screening battery.

From the ICPS recommendations and other discussions given earlier, Fig. G.5 was drawn to demonstrate a hierarchial rank of different data bases considered to be relevant to tumorigenesis and

${ }^{7}$ The International Programme on Chemical Safety (1990) recognized a need

to investigate the value of short-term tests for detecting mutagenic and carcinogenic chemicals. . . conclusion was that the use of chromosome aberration assays in conjunction with the Salmonella mutation test may provide an efficient primary screen for possible new carcinogens. . the mouse bone marrow micronucleus test was confirmed as a robust, sensitive, and reproducible assay. . . overall performance of the rat liver assay for unscheduled DNA synthesis suggested that it could be complementary to the micronucleus test, although certain aspects of the sensitivity and selectivity . . widely advocated assays and tests using Drosophila were concluded to be inappropriate for hazard-assessment purposes. . . . . Chemical carcinogenesis is generally recognized as a multistage process that begins with initiation, usually considered to involve changes in DNA structure leading to mutations, followed by promotion of the lesion to a premalignant state and progression to overt cancer. Thus, the majority of current short-term tests, designed to detect the consequences of DNA interaction, will only respond to chemicals that may induce tumors by a predominantly genotoxic mechanism or induce the initial phase of the carcinogenic process. A feature of the literature on short-term tests for chemical carcinogens is the occasional reference to chemicals, shown to be associated with the induction of cancers in laboratory animals, that consistently fail to be detected in short-term in vitro or in vivo assays for genotoxicity. 
Table G.2. Summary of Bioassay Results for testing/screening of hafnium, tungsten, and zirconium-related compounds

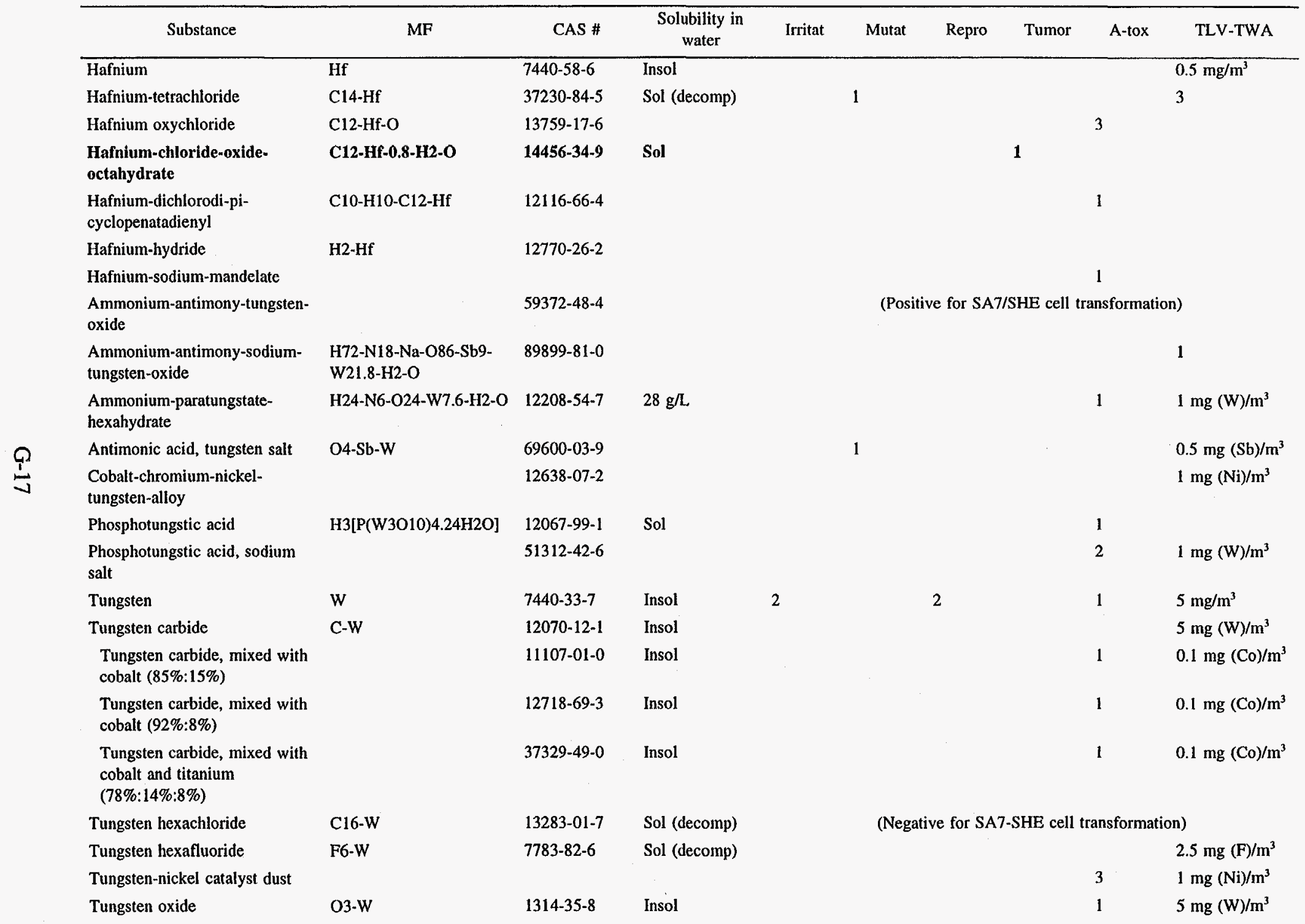


Table G.2 (continued)

\begin{tabular}{|c|c|c|c|c|c|c|c|c|c|}
\hline Substance & $\mathrm{MF}$ & CAS \# & $\begin{array}{l}\text { Solubility in } \\
\text { water }\end{array}$ & Irritat & Mutat & Repro & Tumor & A-tox & TLV-TWA \\
\hline $\begin{array}{l}\text { Tungsten pentacarbonyl } \\
\text { (piperidine) }\end{array}$ & C10-H11-N-O5-W & $31082-68-5$ & & & & & & 1 & \\
\hline $\begin{array}{l}\text { Tungsten, tris(acetonitrile) } \\
\text { tricarbonyl }\end{array}$ & $\mathrm{C} 9-\mathrm{H} 9-\mathrm{N} 3-\mathrm{O} 3-\mathrm{W}$ & $16800-47-8$ & & & & & & 1 & $5 \mathrm{mg}(\mathrm{W}) / \mathrm{m}^{3}$ \\
\hline Tungstic acid & $\mathrm{H} 2-\mathrm{O} 4-\mathrm{W}$ & $11105-11-6$ & Insol & 1 & & & & & $5 \mathrm{mg}(\mathrm{W}) / \mathrm{m}^{3}$ \\
\hline Tungstic acid, disodium salt & $\mathrm{O} 4-\mathrm{W} .2-\mathrm{Na}$ & $13472-45-2$ & & & 4 & 1 & & 10 & $5 \mathrm{mg}(\mathrm{W}) / \mathrm{m}^{3}$ \\
\hline $\begin{array}{l}\text { Tungstic acid, sodium salt, } \\
\text { dihydrate }\end{array}$ & $\mathrm{O} 4-\mathrm{W} .2-\mathrm{Na} .2-\mathrm{H} 2-\mathrm{O}$ & $10213-10-2$ & $410 \mathrm{~g} / \mathrm{L}$ & & 2 & 1 & & 2 & $5 \mathrm{mg}(\mathrm{W}) / \mathrm{m}^{3}$ \\
\hline Tungsten pentaoxide & W2-05 & & Insol & & & & & & \\
\hline Tungsten dioxide & $\mathrm{W}-\mathrm{O} 2$ & $12036-22-5$ & Insol & & & & & & \\
\hline Tungsten oxytetrachloride & W-O-C14 & & Sol (decomp) & & & & & & \\
\hline Tungsten disulfide & W-S2 & & Insol & & & & & & \\
\hline Barium zirconium (IV) oxide & $\mathrm{Ba}-\mathrm{O} 3-\mathrm{Zr}$ & $12009-21-1$ & & & & & & 2 & $5 \mathrm{mg}(\mathrm{Zr}) / \mathrm{m}^{3}$ \\
\hline $\begin{array}{l}\text { Glutamic acid, sodium } \\
\text { zirconium salt }\end{array}$ & & & & & & & & 1 & \\
\hline $\begin{array}{l}\text { Lactic acid, sodium zirconium } \\
\text { salt }(4: 4: 1)\end{array}$ & $\mathrm{C} 12-\mathrm{H} 2 \mathrm{O}-\mathrm{O} 12.4-\mathrm{Na}-\mathrm{Zr}$ & $10377-98-7$ & & & & & & 1 & $5 \mathrm{mg}(\mathrm{Zr}) / \mathrm{m}^{3}$ \\
\hline Lead-titanium-zirconium salt & & $12626-81-2$ & & & & & & 5 & \\
\hline Picramic acid, zirconium salt & $\mathrm{C} 24-\mathrm{H} 16-\mathrm{N} 12-\mathrm{O} 20-\mathrm{Zr}$ & $63868-82-6$ & & & & & & & $5 \mathrm{mg}(\mathrm{Zr}) / \mathrm{m}^{3}$ \\
\hline $\begin{array}{l}\text { Zirconate(2-), hexafluoro-, } \\
\text { dipotassium }\end{array}$ & F6-Zr.2-K & $16923-95-8$ & & & & & & 1 & $2.5 \mathrm{mg}(\mathrm{F}) / \mathrm{m}^{3}$ \\
\hline Zirconium & $\mathrm{Zr}$ & $7440-67-7$ & Insol & & & & & & $5 \mathrm{mg} / \mathrm{m}^{3}$ \\
\hline $\begin{array}{l}\text { Zirconium, bis(acetato- } \\
0, O^{\prime} \text { )oxo- }\end{array}$ & $\mathrm{C} 4-\mathrm{H} 6-\mathrm{O} 5-\mathrm{Zr}$ & 20645-04-9 & Insol & & & & & 2 & $5 \mathrm{mg}(\mathrm{Zr}) / \mathrm{m}^{3}$ \\
\hline Zirconium, bis(nitrato-O)oxo- & $\mathrm{N} 2-07-\mathrm{Zr}$ & $13826-66-9$ & & & & & & 2 & $5 \mathrm{mg}(\mathrm{Zr}) / \mathrm{m}^{3}$ \\
\hline Zirconium tetrachloride & $\mathrm{C} 14-\mathrm{Zr}$ & $10026-11-6$ & Sol (decomp) & & & & & 2 & $5 \mathrm{mg}(\mathrm{Zr}) / \mathrm{m}^{3}$ \\
\hline Zirconium-chloride-hydroxide & $\mathrm{C} 1-\mathrm{H}-\mathrm{O}-\mathrm{Zr}$ & $10119-31-0$ & & 1 & & & & & $5 \mathrm{mg}(\mathrm{Zr}) / \mathrm{m}^{3}$ \\
\hline $\begin{array}{l}\text { Ztrconium-chloride-oxide- } \\
\text { octaphdrate- (Zr-chloride) }\end{array}$ & C12-O-Zr.8-H2-O & $13520-92-8$ & Sol & & 3 & & $\mathbf{1}$ & & $5 \mathrm{mg}(\mathrm{Zr}) / \mathrm{m}^{3}$ \\
\hline $\begin{array}{l}\text { Zirconium, dichloro-di-pi- } \\
\text { cyclopentadienyl- (Zr- } \\
\text { dichloride) }\end{array}$ & $\mathrm{C} 10-\mathrm{H} 10-\mathrm{C} 12-\mathrm{Zr}$ & $1291-32-3$ & & & 2 & & & 1 & $5 \mathrm{mg}(\mathrm{Zr}) / \mathrm{m}^{3}$ \\
\hline Zirconium, dichlorooxo- & $\mathrm{C12}-\mathrm{O}-\mathrm{Zr}$ & $7699-43-6$ & & & & & 1 & 6 & $5 \mathrm{mg}(\mathrm{Zr}) / \mathrm{m}^{3}$ \\
\hline
\end{tabular}


Table G.2 (continued)

\begin{tabular}{|c|c|c|c|c|c|c|c|c|c|}
\hline Substance & MF & CAS \# & $\begin{array}{l}\text { Solubility in } \\
\text { water }\end{array}$ & Irritat & Mutat & Repro & Tumor & A-tox & TLV-TWA \\
\hline Zirconium tetrafloride & $\mathrm{F} 4-\mathrm{Zr}$ & $7783-64-4$ & Sol (decomp) & & & & & & $2.5 \mathrm{mg}(\mathrm{F}) / \mathrm{m}^{3}$ \\
\hline Zirconium gluconate & & $70983-41-4$ & & & & & & 1 & $5 \mathrm{mg}(\mathrm{Zr}) / \mathrm{m}^{3}$ \\
\hline Zirconium glutamate & & & & & & & & 1 & $5 \mathrm{mg}(\mathrm{Zr}) / \mathrm{m}^{3}$ \\
\hline Zirconium hydride & $\mathrm{H} 2-\mathrm{Zr}$ & $7704-99-6$ & NA & & & & & & $5 \mathrm{mg}(\mathrm{Zr}) / \mathrm{m}^{3}$ \\
\hline Zirconium (III) lactate (1:3) & C9-H9-O9-H4-O-Zr & $63919-14-2$ & & & & & & 3 & $5 \mathrm{mg}(\mathrm{Zr}) / \mathrm{m}^{3}$ \\
\hline Zirconium (IV) lactate (1:4) & $\mathrm{C} 3-\mathrm{H} 5-\mathrm{O} 3-\mathrm{Zr}$ & $60676-90-6$ & & & & & & 1 & $5 \mathrm{mg}(\mathrm{Zr}) / \mathrm{m}^{3}$ \\
\hline Zirconium nitrate & $\mathrm{N} 4-\mathrm{O} 12-\mathrm{Zr}$ & $13746-89-9$ & Very sol & & & & & 2 & $5 \mathrm{mg}(\mathrm{Zr}) / \mathrm{m}^{3}$ \\
\hline Zirconium oxide & $\mathrm{O} 2-\mathrm{Zr}$ & $1314-23-4$ & Insol & & & & & & \\
\hline Zirconium (IV) silicate (1:1) & $\mathrm{O} 4-\mathrm{Si}-\mathrm{Zr}$ & $14940-68-2$ & Insol & & & & & & $5 \mathrm{mg}(\mathrm{Zr}) / \mathrm{m}^{3}$ \\
\hline Zirconium-sodium-lactate & C9-H15-Na-O10-Zr & $63904-82-5$ & & & & & 1 & & $5 \mathrm{mg}(\mathrm{Zr}) / \mathrm{m}^{3}$ \\
\hline Zirconium (IV) sulfate (I:2) & O8-S2-Zr & $14644-61-2$ & Very sol & & 1 & 1 & & 3 & $5 \mathrm{mg}(\mathrm{Zr}) / \mathrm{m}^{3}$ \\
\hline $\begin{array}{l}\text { Zirconium, tetrakis }(2,4- \\
\left.\text { pentanedionato- } O, O^{\prime}\right)-\end{array}$ & $\mathrm{C} 20-\mathrm{H} 28-\mathrm{O} 8-\mathrm{Zr}$ & $17501-44-9$ & & & & & & 2 & $5 \mathrm{mg}(\mathrm{Zr}) / \mathrm{m}^{3}$ \\
\hline Zirconyl-citrate & $\mathrm{C} 6-\mathrm{H} 8-07 . \mathrm{x}-\mathrm{Zr}$ & $22830-18-8$ & & & & & & 1 & $5 \mathrm{mg}(\mathrm{Zr}) / \mathrm{m}^{3}$ \\
\hline Zirconyl-sodium-sulfate & $\mathrm{Na2}-\mathrm{O} 18-\mathrm{S} 4-\mathrm{Zr} 2$ & $24735-35-1$ & & & & & & 2 & $5 \mathrm{mg}(\mathrm{Zr}) / \mathrm{m}^{3}$ \\
\hline Zirconium carbide & $\mathrm{Zr}-\mathrm{C}$ & & Insol & & & & & & \\
\hline
\end{tabular}

Note: Bold indicates tumorigenic test data: insol means $\leq 0.01 \mathrm{~g} / \mathrm{cc}$ 


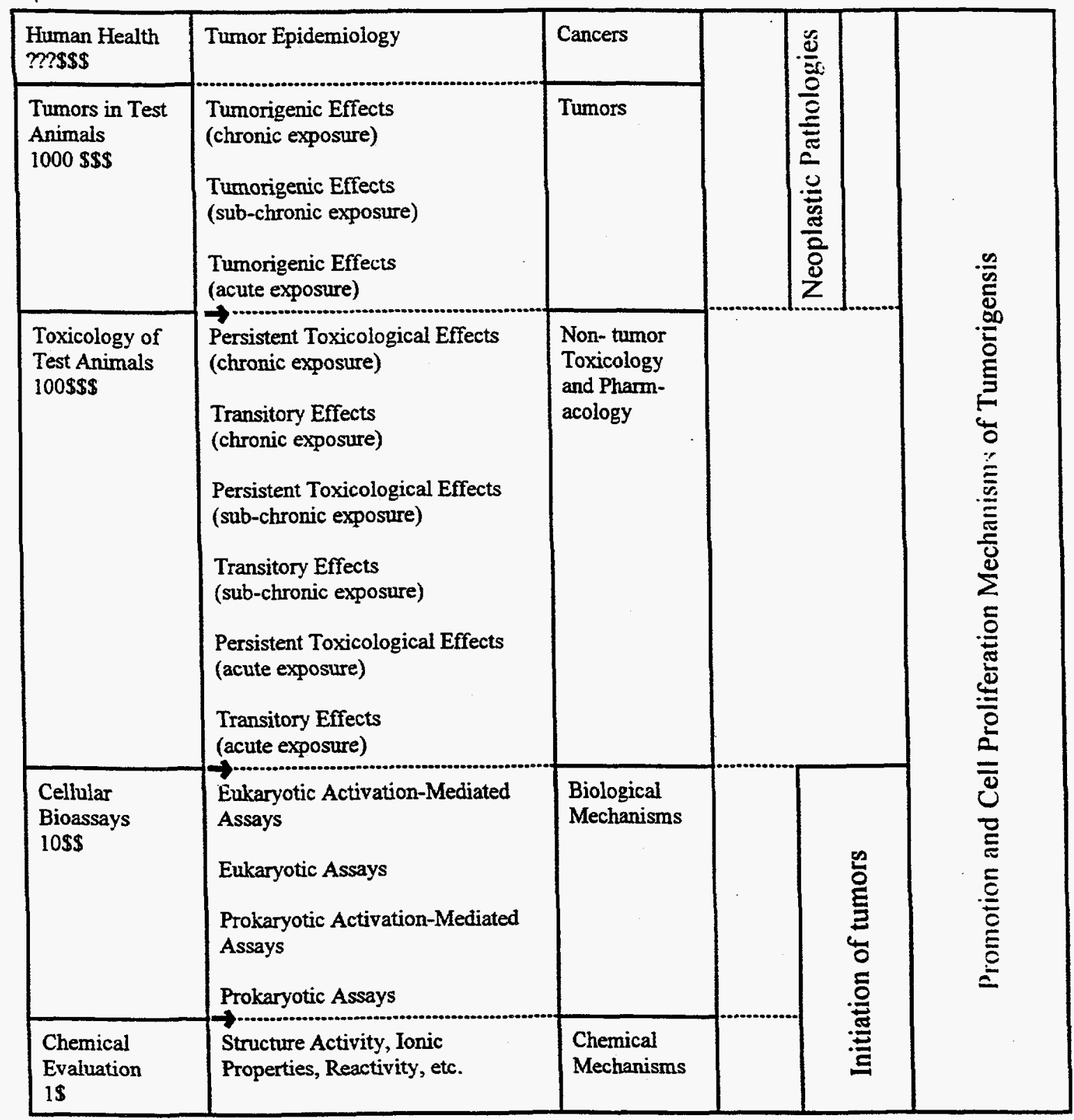

Fig. G.5. Hierarchial rank of different data bases considered to be biologically relevant to tumorigenesis and human risks.

other chronic diseases to humans. Availability of data is usually inversely related to the number of dollar signs subjectively assigned to each category. If regulatory policy is based exclusively on validated tumor data, then many potentially serious hazards are likely to go uncontrolled. In contrast, if protection of human health is driven by data on carcinogenic initiation, then compounds such as saccharine and ethyl alcohol are carcinogenic in the same sense as benzene and 2,3,7,8-TCDD, and policy decisions will be fraught with inaccuracies, high costs, and imprecisions from compound to compound. 
In the extrapolation of both dose and response to humans from a particular bioassay, using traditional mathematical models and many assumptions, lack of knowledge and uncertainty pose barriers in the quest for accuracy. Only if the extrapolation distance is very small does one have any confidence of accuracy.

The traditional data-sparse, assumption-intensive methods based on models used to extrapolate both dose and response have neither the accuracy nor the precision needed for reliable assessments of risk. In Fig. G.5, different classes of bioassays (sorted according to complexity or relevance of biological effects illustrated in Fig. G.4) are subjectively ranked in a hierarchial tier as seen by the text at the left of the figure. If the ultimate goal is risk assessment for potentially exposed human populations, then one or more different bioassays may be chosen as a starting point. The sensitivity of the bioassay chosen will be drawn from a sensitivity probability distribution of similarly based and/or procedurecontrolled bioassays and may or may not represent the central response or sensitivity of its own class.

From such a starting point, mathematical models and theories predicated on the basis of biological mechanisms can be used to extrapolate the response trajectory (shown as a dashed pathway in Fig. G.6) that might be observed in a different bioassay (or even a different sex or strain within the same experimental context). The dashed projections are force vectors from models and assumptions: they are curved from the "true" center-of-risk aim-point to indicate the mechanism(s) of molecular toxicological action for any particular compound of concern that is (are) usually unknown and probably act as combinations of directional force vectors, because each compound may be mediated by multiple mechanisms, each of which may change in relative importance depending upon the dose-ratehost-environment-related factors. Obviously, the greater the extrapolation distance across reaches of biological structure, the larger the probable error in the inherently uncertain process of extrapolation.

Historical studies have demonstrated that the choice of mathematical extrapolation model for a particular set of experimental data on a chosen pollutant can cause variations in excess of ten orders of magnitude at low doses. As illustrated in this figure, there may be comparable degrees of added uncertainty due to selection of a particular biological experiment as a starting point and subsequent projection of its dose response to different levels of biological structure.

The conclusion from this illustration is that mathematical extrapolation of biologically dependent events is intrinsically unstable. As experienced with the NTP's two-year rodent test results with four different test populations (viz., male and female test groups of rats and mice), many compounds are subject to sex- and species-related effects (see Fig G.1). Additionally, strain-related effects will also contribute to uncertainty as could variability resulting from genetic diversity within typical human populations. Furthermore, many assessments of risk are still weakened by reliance on experimental studies conduced with a pure compound tested in a laboratory-restricted protocol where extraordinary efforts are made either to remove or to minimize all other toxic/mutagenic exposures.

Protocols of most relevance to human risk/hazard assessment should be designed to test the amplifying potency of a test insult on a bioassay subject that is jointly exposed to other low-dose mutagens and carcinogens. The differential response between the positive control experiments without potentiating coexposures and their counterpart protocols involving the amplifying effects of the test insult on similarly-treated positive controls should more realistically address potential risk to hypothetically exposed humans. 
Risk to Humans

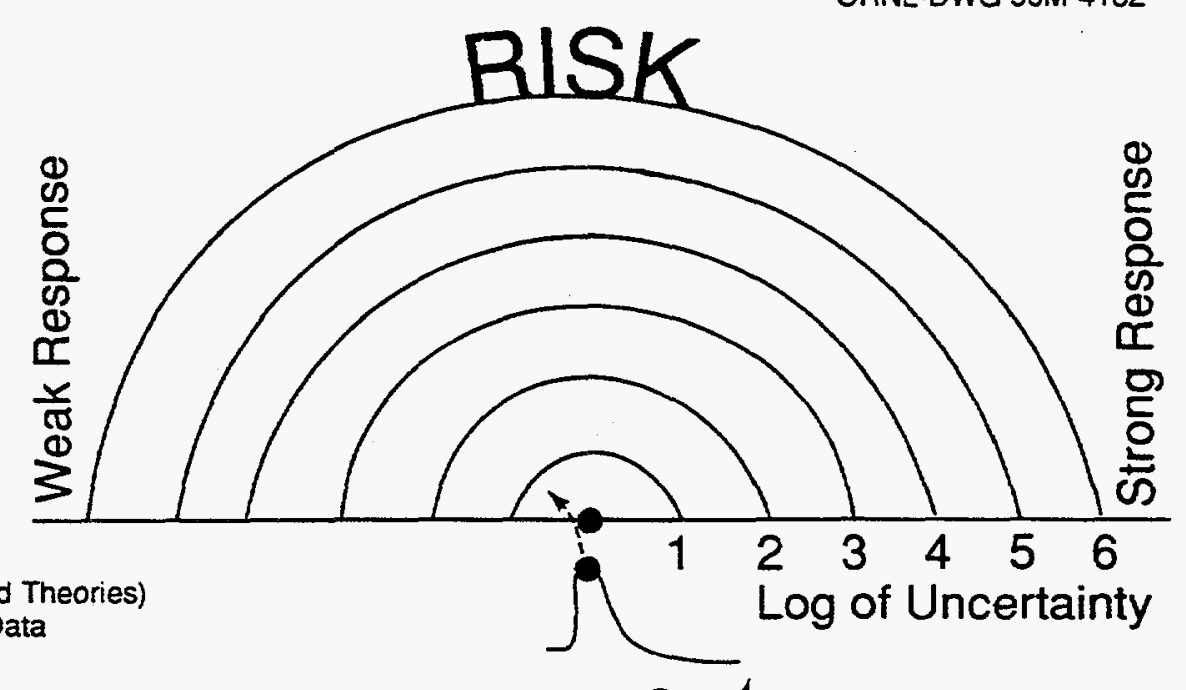

(Models and Theories)

Epidemiological Data

(Models and Theories)

NTP 2 Year Rodent Tests

(Models and Theories)

Tumors in Test Animals

(Models and Theories)

Pharmacological Experiments

(Models and Theories)

Non-Tumor Toxicology Experiments

(Models and Theories)

Host-Mediated Eukaryotic Experiments

(Models and Theories)

Eukaryotic Experiments in Culture -

(Models and Theories)

Prokaryotic Experiments in Culture

(Models and Theories)

SAR, $e^{-}$, and Reactivity Evaluations
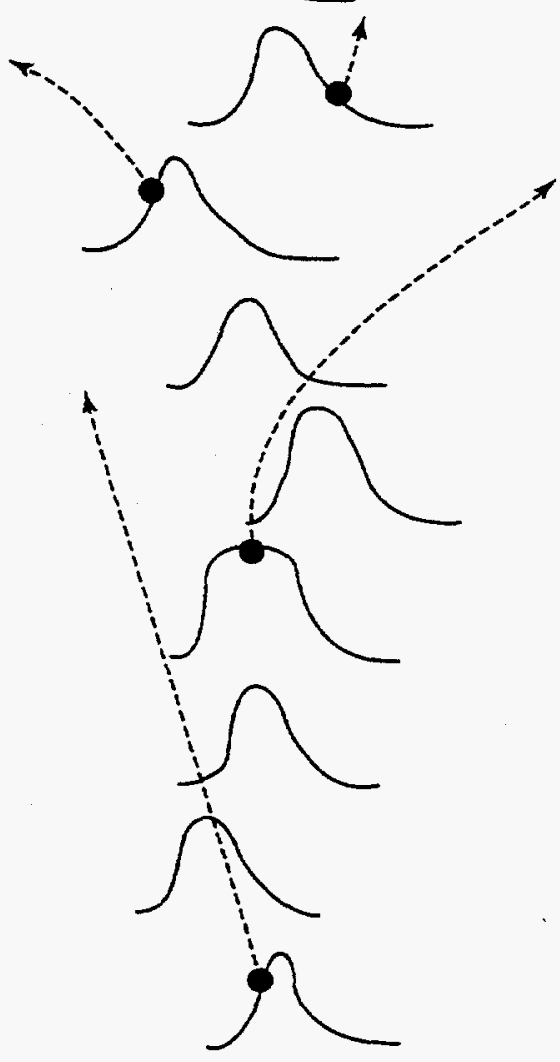

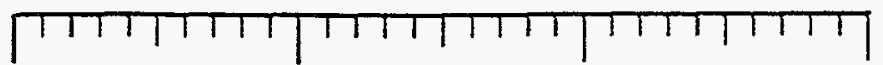

Fig. G.6. Variability of different bioassay methods and extrapolation models. Historical approaches to risk analysis are diagramatically illustrated here to demonstrate that "data sparse, assumption-intensive methods" have neither the accuracy nor the precision needed for reliable assessments of risk. 
The tradition of testing every component of concern in rigorously designed bioassays where all other known risk factors are censored to determine if each and every insult of concern intrinsically carries each and every aspect of carcinogenic initiation and promotion is actually misdirected when in reality all humans are exposed to mixtures of radiological and chemical agents. These can share mutagenic properties and promoting potencies through mixed-field or sequential exposures incurred through environmental, occupational, lifestyle, and/or heredity-related risk factors (Ames 1990 and Emerit 1987). Models for risk/hazard from multiple exposures may be based on the classic treatments for independent or joint toxic actions (Finney 1971, Jones 1995).

\section{G.5 RELATIVE POTENCY}

In the sense of the Boltzman equation used for radiation transport, the biological response can be indicated symbolically by:

$$
\text { Response }=F(S, T, G, A, D, M, T F, t e[t], t f[t]) d t \text {, }
$$

where $F$ is a function of source characteristics $(S)$; transported substance (T); geometrical aspect $(G)$ of the agent involved including size and length-to-diameter aspect ratio; absorption into the human body (A); distribution to organs and tissues (D); metabolism (M); toxicity factor (TF); chronological time (t); exposure time (te[t]); and total follow-up time or period of risk from the start of the first exposure $(\mathrm{tf}[\mathrm{t}])$. This symbolism is used mainly to demonstrate that many considerations are involved in assessments of risk. Because many of the considerations fundamental to risk assessment are multiplicative in nature, one can not develop meaningful comparisons unless data are sufficient to characterize each factor or combination of factors so involved-for example, oral $\mathrm{LD}_{50}$ data in animals intrinsically span considerations of $\mathrm{A}, \mathrm{D}, \mathrm{M}$, te, and tf.

A common scale would enable direct comparisons of radiological hazards deriving from depleted uranium with toxicological hazards deriving from tungsten-related compounds. As patterned after recommendations of Committee 17 of the Environmental Mutagen Society, all evaluations are based on the common observation that "no system by itself meets all the requirements of an ideal system, but the available systems collectively provide important information." Two types of numerical comparisons are considered: (1) placement of the compounds of interest on a relative potency scale used to rank hundreds of other chemical compounds with substances such as saccharine, ethyl alcohol, and vinyl chloride at the low end and aflatoxin $B_{1}$ and 2,3,7,8-tetrachlorodibenzo-p-dioxin $(2,3,7,8-\mathrm{TCDD})$ at the high end, and (2) use of available bioassay results to establish several direct common-scale quantitative bridges between chemical toxicity and radiotoxicity.

Historically, chemically inert substances have been treated as non-hazardous to biological systems, but occupational exposures acquired over decades have now shown that most such substances can induce a spectrum of toxicological effects including carcinogenesis. The chemically inert substances of current concern include: asbestos, calcium, coal dust, copper, copper sulfate, fiberglass, gold, graphite, iron and ore, platinum, quartz, sand, silica, silver, stainless steel, talc, tin, and teflon.

Great expense, long delays, and much uncertainty are intrinsic to carcinogenic testing-even that associated with the rigorous National Toxicology Program (NTP) two-year dosing schedules used for mice and rats. That degree of testing is inappropriate for hazard screening and may not do the job as well as simple hazard screening based on a large battery of less expensive bacterial and mammalian 
cell bioassays. ${ }^{8}$ However, no consensus has been reached regarding the specific tests that should make up a standard screening battery.

Finney in Probit Analysis (1971) reviewed three types of joint toxic action: (1) independent if poisons or drugs have different modes of action; (2) similar if one poison or drug can be substituted in a constant proportion (at all doses) for the other; and (3) synergistic if the effectiveness of the mixture cannot be assessed from that of the ingredients. ${ }^{9}$ Processes of independent and similar action are shown in Fig. G.7.

One caution is that some combinations of compounds and/or specific concentrations of those compounds (or the respective times of administration of the individual components of the multi-insult experiment) may initially appear to produce a dose-response synergism; however, if considered more carefully, the perceived mechanism may not be supported by more realistic choices for the compoundspecific dose-response model. Overall, the concern about synergistic reactions is not well supported by published experimental data-especially in the low-dose range. However, it does appear that highly selected experimental protocols may sometimes be tuned to demonstrate a synergistic behavior-usually such observations are based on assumed dose-response models and are not defensible at the molecular level.

${ }^{8}$ This concept was recognized by the International Programme on Chemical Safety (1990)

to investigate the value of short-term tests for detecting mutagenic and carcinogenic chemicals. . conclusion was that the use of chromosome aberration assays in conjunction with the salmonella mutation test may provide an efficient primary screen for possible new carcinogens. . the mouse bone marrow micronucleus test was confirmed as a robust, sensitive, and reproducible assay. . . overall performance of the rat liver assay for unscheduled DNA synthesis suggested that it could be complementary to the micronucleus test, although certain aspects of the sensitivity and selectivity . . . widely advocated assays and tests using drosophila were concluded to be inappropriate for hazard-assessment purposes. . . . . Chemical carcinogenesis is generally recognized as a multistage process that begins with initiation, usually considered to involve changes in DNA structure leading to mutations, followed by promotion of the lesion to a premalignant state and progression to overt cancer. Thus, the majority of current short-term tests, designed to detect the consequences of DNA interaction, will only respond to chemicals that may induce tumors by a predominantly genotoxic mechanism or induce the initial phase of the carcinogenic process. A feature of the literature on short-term tests for chemical carcinogens is the occasional reference to chemicals, shown to be associated with the induction of cancers in laboratory animals, that consistently fail to be detected in short-term in vitro or in vivo assays for genotoxicity.

${ }^{9}$ Synergism is not discussed here because particular ingredients may alter absorption into the body; vascular distribution; detoxification; elimination; or organ sensitivity according to either sensitizing or desensitizing reactions. Possible synergisms have motivated terms in dose-response models since the 1930s, but those efforts, although impressive, have actually contributed little substance beyond Finney's simple observation that for synergism "the effectiveness of the mixture cannot be assessed from that of the individual ingredients." Thus, when synergistic interactions are expected, the mixture should be tested as if it were a new compound. This may require sample concentration, but that is analogous to the typical process of testing a new compound and then using the test results for environmental and/or occupational settings. 


\section{INDEPENDENT ACTION:}

(a)

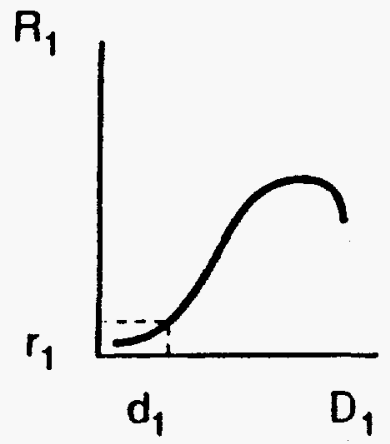

(b)

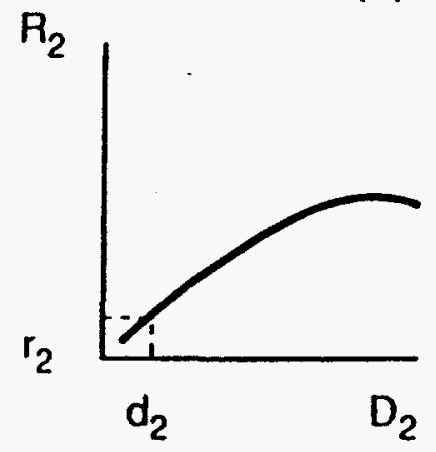

$=r_{1}+r_{2}$

\section{JOINT ACTION:}
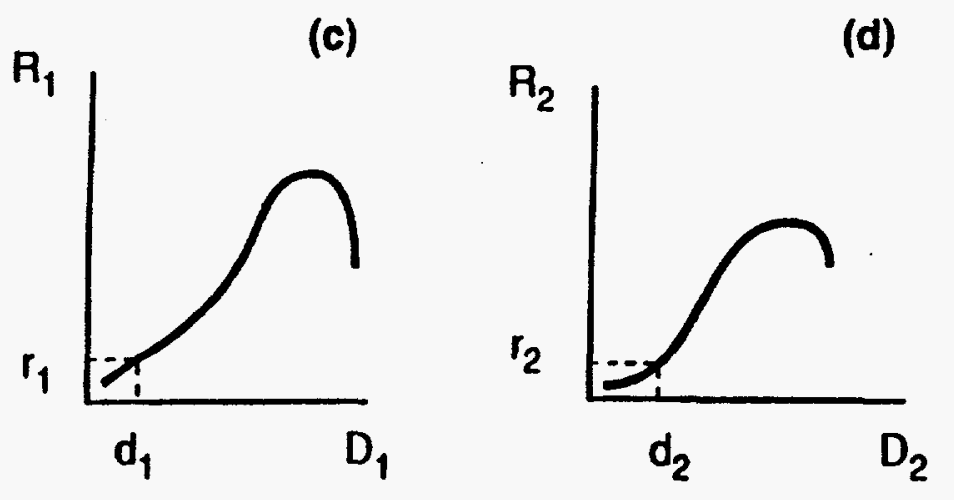

(e)

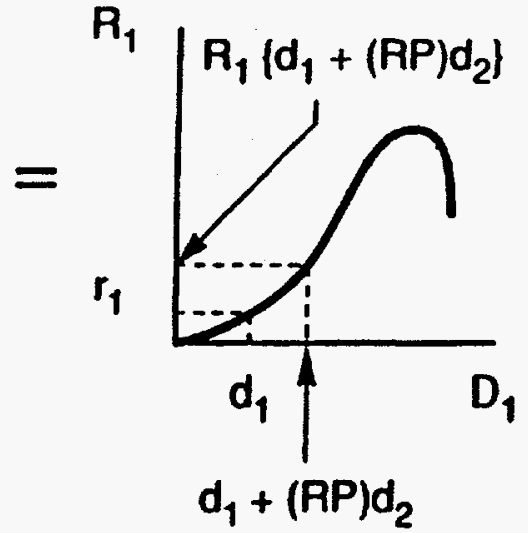

Fig. G.7. Chemicals having independent mechanisms of action lead to risk-additive models while chemicals having joint action on a particular response or mechanism lead to dose-additive models.

As illustrated in Fig. G.7, the Finney concept of independent action leads to risk-additive models, whereas similar action or joint toxicity leads to dose-additive models. As formulated by Finney, joint action is logically equivalent to the simple relative potency approach which makes the toxicology of complex mixtures predictable from its ingredients by application of the simple harmonic mean concept. In addition, the relative potency concept can be used to make predictions from bioassay results compared with matched results from one or more suitable reference compounds because "each compound can be substituted in potency-adjusted-doses for any of the other compounds."

To obviate some of the problems of uncertainty and minimize the impact of others associated with the historical dose-response extrapolation models, a "data-intensive, assumption-sparse" approach based on relative potency comparisons between chemical compounds may be warranted as illustrated in Fig. G.8. The accuracy of such a relative (hazard or potency) process for human health risk is difficult-perhaps even impossible- to establish in an absolute sense, but numerical precision from 


\section{Relative Hazard Potential}

Risk to Humans

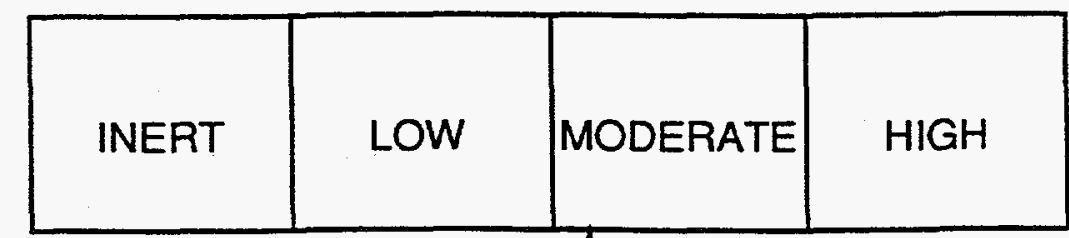

(Models and Theories)

Epidemiological Data

(Models and Theories)

NTP 2 Year Rodent Tests

(Models and Theories)

Tumors in Test Animals

(Models and Theories)

Pharmacological Experiments

(Models and Theories)

Non-Tumor Toxicology Experiments

(Models and Theories)

Host-Mediated Eukaryotic Experiments

(Models and Theories)

Eukaryotic Experiments in Culture

(Models and Theories)

Prokaryotic Experiments in Culture

(Models and Theories)

SAR, $\mathrm{e}^{-}$, and Reactivity Evaluations

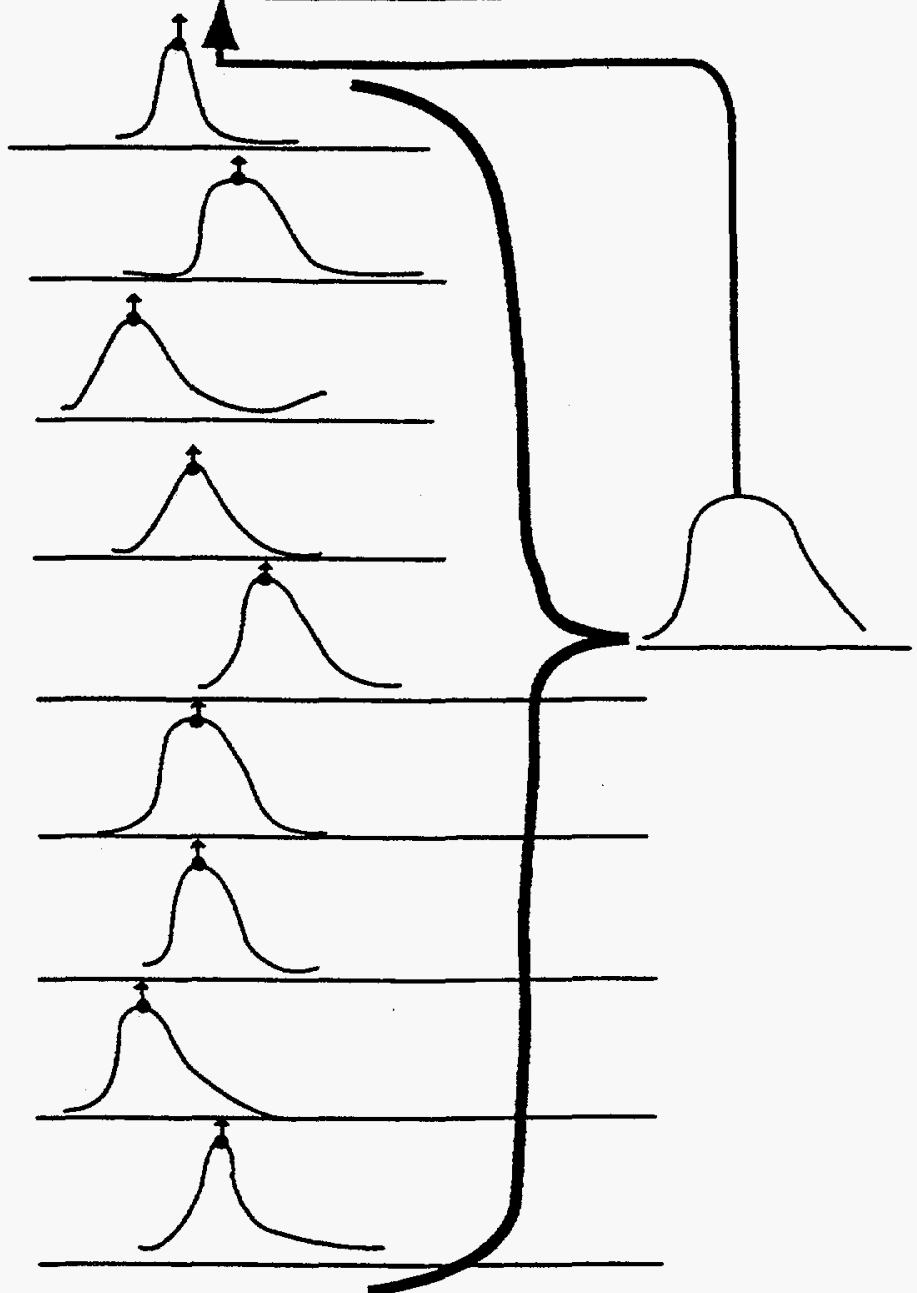

Fig. G.8. Data from many chemical, physical, and biological models can be used for hazard assessment. Estimates of "relative potency" can supplement or replace extrapolation models based on datasparse, assumption-intensive analyses as illustrated in Fig. G.1.

compound to compound is intrinsic because of the data-rich process of comparing dose-response data from an interviewing compound of concern with dose-response data for one or more carefully chosen reference compounds whose effects in bioassays and on human populations are well known.

A method of comparative toxicities can be used to estimate route-, rate-, and duration-specific acceptable exposures from a new (or inadequately tested) hazard relative to a reference exposure or agent of risk for which much human, animal, and cell culture data are available. There may be much 
uncertainty as to whether the particular hazard of concern can, in and of its own actions, actually cause a complex disease such as cancer, but the magnitude of the potential hazard can be estimated from an array of relative potency factors derived from various bioassays.

An estimate of the relative potency (RP) of hazard " $\mathrm{H}$ " compared with an agent or exposure of risk " $R$ " can be obtained by comparing the doses $\left(D_{H}\right.$ and $\left.D_{R}\right)$ required to cause the same level of response in a particular biological test model considered to be relevant to the disease of interest. For example, if a certain cell transformation assay or the mouse bone-marrow micronucleus test is thought to mimic certain important aspects of carcinogenic initiation, then an estimate of the relative potency can be made from $R P=\left(D_{R} / D_{H}\right)$. Likewise, if cell proliferation is considered to be related to carcinogenic initiation and/or promotion, then tests that measure cytotoxicity, toxic hyperplasia, acute toxicity to animals, DNA damage, etc. may be used to extend the array of RP estimates relevant to the hazard of interest.

Dose units between radiological and chemical hazards are quite distinct. A strategy for direct comparison of risk from radiological exposures relative to risk from an alternative chemical exposure can be demonstrated as shown in Table G.3. If $\mathrm{R}$ is used as the effective dose of concern from depleted uranium and $C$ is the chemical dose of concern from tungsten, then these two insults are expected to have differential degrees of toxicity/risk/hazard and that differential effect can be estimated directly from the classical hazard index formulation.

The typical hazard index for either radiological or chemical applications involves comparison of an exposure or dose of concern (numerator of a ratio) to a regulation, guideline, or reference exposure expressed in the same units (denominator of the ratio). The denominator of the two hazard index quantities should be associated with equal levels of response in the same bioassay. Thus, a natural common scale is established between radiological and toxicological exposures. Because the hazard index ratio is without dose units (i.e., $\mathrm{R} / \mathrm{ri}$ for radiation and $\mathrm{C} /$ ci for chemicals, where ri is identical to ci in the bioassay of choice) a hazard index for depleted uranium can be compared directly with its counterpart for tungsten-at least in theory.

Radiation comparisons, in general, are readily established because the dosimetry concepts apply to all ionizing radiations of different linear energy transfer (LET) or ionic stopping power qualities. Furthermore, radiation has been tested in various bioassays including many of those listed in the RTECS for assessments of chemical toxicity.

For chemical compounds, as with radiation, it is only required that the hazard index be unitless. Therefore, for in vitro tests the insult may be expressed in concentration as with $\mathrm{mg} / \mathrm{L}$ or in the form of Haber's dose based on the product of concentration and exposure time. Similarly, for doses to in vivo targets, the insult can be expressed as $\mathrm{mg}$ of insult per $\mathrm{kg}$ of body weight or alternatively as the integral of organ concentration as a function of time, over time. Either can be used, and the choice may depend upon the available data and whether realistic or upper limit estimates are required.

From the current philosophy that compensatory cell proliferation is associated with all stages of carcinogenesis, as reviewed earlier, and that most in vitro bioassays directly address mutagenic potential for alterations in the DNA of a cell or demonstrate through clastogenic action that a compound has left its signature in the tissues at risk, one would be somewhat pressed to dismiss any of the usual bioassay results as being irrelevant to potential hazards from tungsten. It is highly desirable to have a wide diversity of bioassay results for both radiological and toxicological hazards. 
Table G.3. Hazard index examples showing that rad-dose $R$ and chem dose $\mathbf{C}$ may be based on doses ri and ci, which induce the same level of effect

on a given endpoint ${ }^{n}$

\begin{tabular}{clcccc}
\hline No. & \multicolumn{1}{c}{ Toxicological/radiological criteria } & $\begin{array}{c}\text { Rad- } \\
\text { dose }\end{array}$ & $\begin{array}{c}\text { Chem- } \\
\text { dose }\end{array}$ & $\begin{array}{c}\mathrm{Haz} \\
\text { Index } \\
\text { (rad) }\end{array}$ & $\begin{array}{c}\text { Haz } \\
\text { (chem) }\end{array}$ \\
\hline 1 & LD50 & $\mathrm{r} 1$ & $\mathrm{c} 1$ & $\mathrm{R} / \mathrm{r} 1$ & $\mathrm{C} / \mathrm{c} 1$ \\
2 & Cancer (e.g., 1/100,000 per lifetime) & $\mathrm{r} 2$ & $\mathrm{c} 2$ & $\mathrm{R} / \mathrm{r} 2$ & $\mathrm{C} / \mathrm{c} 2$ \\
3 & Survival Hela Cells & $\mathrm{r} 3$ & $\mathrm{c} 3$ & $\mathrm{R} / \mathrm{r} 3$ & $\mathrm{C} / \mathrm{c} 3$ \\
4 & Survival Hamster Cells & $\mathrm{r} 4$ & $\mathrm{c} 4$ & $\mathrm{R} / \mathrm{r} 4$ & $\mathrm{C} / \mathrm{c} 4$ \\
5 & Survival Mouse Lymphoma Cells & $\mathrm{r} 5$ & $\mathrm{c} 5$ & $\mathrm{R} / \mathrm{r} 5$ & $\mathrm{C} / \mathrm{c5}$ \\
6 & Survival Mouse Marrow Spleen Cells & $\mathrm{r} 6$ & $\mathrm{c} 6$ & $\mathrm{R} / \mathrm{r} 6$ & $\mathrm{C} / \mathrm{c} 6$ \\
7 & Mammalian Cell Mutation & $\mathrm{r} 7$ & $\mathrm{c} 7$ & $\mathrm{R} / \mathrm{r} 7$ & $\mathrm{C} / \mathrm{c} 7$ \\
8 & Chromosome Aberrations in Human & $\mathrm{r} 8$ & $\mathrm{c} 8$ & $\mathrm{R} / \mathrm{r} 8$ & $\mathrm{C} / \mathrm{c} 8$ \\
& Lymphocytes & & & & \\
9 & Chromosome Aberrations in Hamster Liver Cells & $\mathrm{r} 9$ & $\mathrm{c} 9$ & $\mathrm{R} / \mathrm{r} 9$ & $\mathrm{C} / \mathrm{c} 9$ \\
10 & Sister Chromatid Exchanges in Mammalian Cells & $\mathrm{r} 10$ & $\mathrm{c} 10$ & $\mathrm{R} / \mathrm{r} 10$ & $\mathrm{C} / \mathrm{c} 10$ \\
11 & Mouse Marrow Micronucleus Test & $\mathrm{r} 11$ & $\mathrm{c} 11$ & $\mathrm{R} / \mathrm{r} 11$ & $\mathrm{C} / \mathrm{c} 11$ \\
12 & Mutations in Drosophila Sperm & $\mathrm{r} 12$ & $\mathrm{c} 12$ & $\mathrm{R} / \mathrm{r} 12$ & $\mathrm{C} / \mathrm{c} 12$ \\
13 & Cytotoxicity in E. Coli & $\mathrm{r} 13$ & $\mathrm{c} 13$ & $\mathrm{R} / \mathrm{r} 13$ & $\mathrm{C} / \mathrm{c} 13$ \\
14 & Mutations in E. Coli & $\mathrm{r} 14$ & $\mathrm{c} 14$ & $\mathrm{R} / \mathrm{r} 14$ & $\mathrm{C} / \mathrm{c} 14$ \\
\hline
\end{tabular}

${ }^{\sigma}$ Because radiation dose and chemical dose have no common units, direct comparisons must depend upon establishing a common level of response in a particular bioassay. An exposure to radiation $\mathbf{R}$ (units of dose) can be compared with an exposure to a chemical compound $C$ (units of dose) if the rad dose is first compared with a dose (ci) causing the same effect. Thus, two unitless hazard index values can be compared directly. If this process is continued for all biological endpoints of reference (where data permit) as indicated in tabular form, then radiological hazard can be compared directly with chemical hazard and ranges of uncertainty also can be associated with conventional estimates of central tendency and range.

From such an array of independent and complementary estimates of the hazard index, one can easily compare the mean values and variances of the log-normal distributions and whether the bioassays or data considered relevant to carcinogenesis are supported by the other bioassays relevant to initiation or promotion. It is far more informative to compare frequency distributions than to implement the common approach of comparing point estimates that may have been badly distorted by the use of assumptions and linear extrapolation models.

\section{G.5.1 Rapid Screening of Hazard (RASH)}

The Environmental Monitoring Plan for the U.S. Synthetic Fuels Corporation (1983) stated that the Corporation should have the burden of justifying the need to monitor specific unrelated substances and of providing threshold values above which those substances must be monitored. Somewhat overlapping in time, the U.S. Air Force sponsored the development of a Hazard Assessment Rating Methodology 
(HARM) that finally became known at the Defense Priority Model (Department of Defense 1987 and 1989) for site-specific screening of the Installation Restoration Program. Both of these activities required screening criteria based on toxicological and/or carcinogenic risks for compounds that were unregulated, and for the most part, ignored by expert committees or regulatory agencies at that time.

Based on observed linear relationships between carcinogenic risk and either cytotoxicity or compensatory cell proliferation for both ionizing radiations (Jones et al. 1979, Jones 1984) and carcinogenic chemicals (Jones et al. 1983), a Rapid Screening of Hazard (RASH) method was proposed (Jones et al. 1985). During the next decade, many compounds and mixtures, both carcinogenic and non-carcinogenic, were evaluated by the RASH-based relative potency concept. From that experience, it was observed that the median relative potency from a wide array of bioassays tended to accurately characterize the toxicological and carcinogenic potency to a far greater accuracy than the \pm 100 factor originally anticipated. In fact, for most compounds tested in 20 or more bioassays, accuracy of 2- to 5-fold was common (based on compound-specific comparisons with recommendations by Agency-sponsored expert committees) and after 5 years of usage, the RASH method was described as being potentially useful to forecast or predict new and/or changing regulatory criteria (Jones 1991 and 1993, Jones et al. 1991). Additional experience as summarized in the journal literature is cited as RASH 19XX. Because RASH has been documented many times, it will not be repeated here except for noting the differences between the tedious hand calculations associated with RASH and the Prototype personal-computer version (CRASH). If a more in-depth discussion is desired, readers should refer to the citations "RASH 19XX" in the list of references.

\section{G.5.2 Personal-Computer RASH (CRASH)}

In most regards, the CRASH code was designed to be as similar to the original RASH method (Jones et al. 1988) as possible. Thus, the goal was to match each bioassay available for the interviewing or test compound with a similar result for one and only one reference compound. Reference compounds included B(a)P as a primary standard and several secondary standards that were sometimes varied from study to study. Whenever a test result for the interviewing compound was matched successfully, the calculation moved on the next bioassay without considering whether matches with other secondary standards were possible. Because only humans can adjust to fuzzy logic involving constant changes from compound to compound, it was found that computerized matches should have fixed and predictable logical structure with regard to secondary standards. Since PCs are almost infinitely faster than humans at matching bioassays and computing relative potency ratios, more comparisons between the interviewing compound and the several reference compounds provide greater accuracy-given that the products of many bioassays matched to many different primary reference compounds are used correctly. Used incorrectly, an impressive degree of precision can be achieved but the goal of accuracy missed.

It was recognized from the beginning that the RASH method did not necessarily need to match compounds according to their mechanisms of action because the definition is analogous to that of work, viz., force applied and work achieved. However, the chemical's structure often controls the selection of the bioassays used to test its potency and it is readily seen that inorganic compounds are frequently tested in bioassays and protocols that may be uncommon to organic compounds. In addition, a study in review for publication (Jones and Basavaraju, Submitted) demonstrated that hormones such as estradiol, estrogen, and DES are frequent outliers on scatter plots of potency comparisons for a typical list of occupational and environmental pollutants. Thus, if hormone-like compounds are used as reference compounds, they do not provide a high degree of correlation when 
the same list of chemical compounds is compared with another reference compound. Hormone action simply seems to induce a noise level that overwhelms any coherent signal needed for parametric comparisons, although the fidelity of rank order seems to be preserved (Jones and Basavaraju, Submitted).

Because of the two forces of uncertainty as described above, the CRASH analyses will typically be based on matches of all of the bioassay results with each of five reference compounds used one by one. The median relative potency is taken for the interviewing compound relative to a particular reference compound. Each median is specific to the potence of the reference compound selected. Multiple reference compounds lead to relative potency values that must be normalized to a common scale before further analysis can proceed. This is accomplished by normalization so that B(a)p has unit potency for each of the other reference compounds used. Finally, the overall estimate of a chemical's toxicity is taken as the median estimate of the medians for the five different reference compounds. This helps to minimize atypical test results in both the interviewing and the reference compounds and will be seen to contribute both accuracy and precision in the tables and figures that follow. It is imperative to match in this manner or in a logically equivalent manner because if relative potencies are computed indiscriminantly, then Ames tests and $\mathrm{LD}_{50}$ estimates will, because of their relative abundances, dominate the results. This uncharacteristic proliferation of 'excessive matches' typically leads to great precision but is essentially equivalent to using only two or three distinct bioassays to characterize a compound and may result in inaccuracy expressed in orders of magnitude. Results may be particularly erroneous if short-term mutation tests dominate the matching process and potency evaluations are dominated, for example, by Ames tests with and without activation.

The logic underlying Fig. G.8 will now be demonstrated for 67 compounds from the Gold et al. (1984) data base on test results for the NTP 2-year dosing protocols to rodents. Similar methods will then be used to describe the precision and accuracy aspects for a common scale based on relative potency and the quantitative bridges between radiobiological toxicity of depleted uranium and toxicological hazards associated with tungsten.

\section{G.5.3 Comparison of CRASH-Based Relative Potency Predictions with the Gold Data Base}

Jones (1995) evaluated the toxicological potency of $2,3,7,8$-TCDD relative to 100 other compounds. This worked focused on a relative potency analysis of in vitro and in vivo test data. In that comparison, 101 compounds were considered. The list was comprised of common environmental contaminants, many of which were carcinogens and all of which were reasonably well tested in a variety of bioassays so that adequate numbers of matches could be obtained for the other 99 compounds relative to both $\mathrm{B}(\mathrm{a}) \mathrm{P}$ and $2,3,7,8-\mathrm{TCDD}$. Of the 101 compounds, 55 were tested in both rats and mice with 33 of them producing positive test results in both species (the other 22 tested positive in one species as seen in Fig. G.1). Another 26 compounds produced positive test results in one species but were not tested in the second species. Of the 101 compounds, only 20 were not found in the Gold data base including compounds that tested negative in both species and compounds not yet tested in the NTP protocol. Of the 81 compounds listed in the Gold et al. data base (1989), 67 were included in the Gold et al. published data base of 1984 which listed the dose tested. For this analysis of precision and accuracy of relative potency, that list of 67 compounds (Table G.4) was used for analysis because comparisons could be made with relative potencies estimated from RASH, $\mathrm{TD}_{50}$ estimates, and actual doses tested in the two-year protocols. 


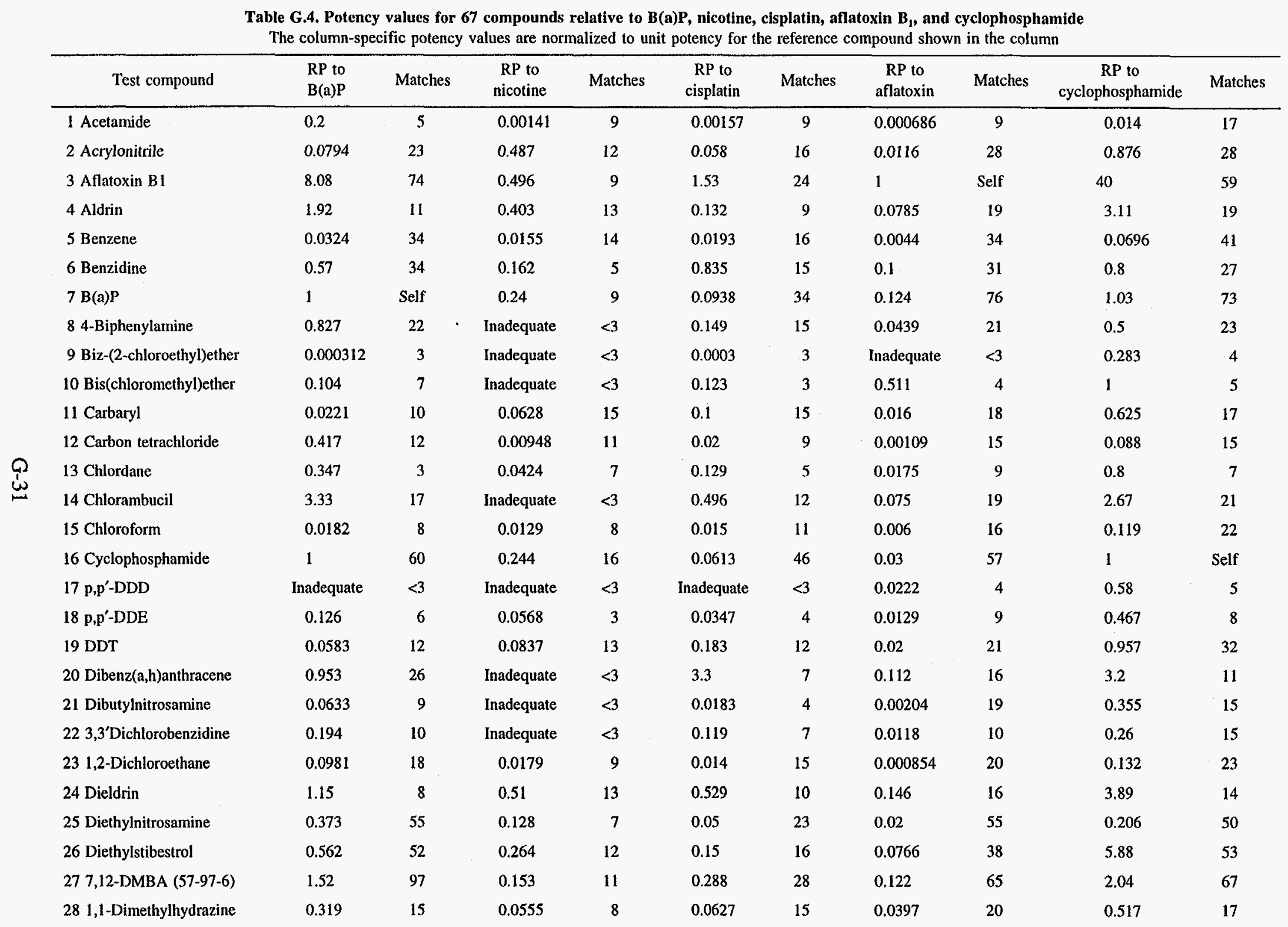


Table G.4 (continued)

\begin{tabular}{|c|c|c|c|c|c|c|c|c|c|c|}
\hline Test compound & $\begin{array}{l}\text { RP to } \\
\text { B(a)P }\end{array}$ & Matches & $\begin{array}{l}\mathrm{RP} \text { to } \\
\text { nicotine }\end{array}$ & Matches & $\begin{array}{l}\text { RP to } \\
\text { cisplatin }\end{array}$ & Matches & $\begin{array}{c}\mathrm{RP} \text { to } \\
\text { aflatoxin }\end{array}$ & Matches & $\begin{array}{c}\text { RP to } \\
\text { cyclophosphamide }\end{array}$ & Matches \\
\hline 29 Dimethylnitrosamine & 0.34 & 75 & 0.862 & 7 & 0.27 & 23 & 0.0498 & 72 & 0.995 & 61 \\
\hline $30 \mathrm{p}$-Dioxane & 0.0005 & 7 & 0.0128 & 4 & 0.00574 & 5 & 0.0000341 & 11 & 0.00634 & 10 \\
\hline 31 Diphenylnitrosamine & 0.0202 & 17 & 0.0063 & 5 & 0.00476 & 9 & 0.00263 & 17 & 0.11 & 21 \\
\hline 32 ENNG (4245-77-6) & 0.227 & 24 & Inadequate & $<3$ & 2.58 & 6 & 0.0375 & 13 & 14.4 & 10 \\
\hline 33 Estradiol & 0.52 & 11 & 705 & 6 & 0.367 & 5 & 0.0918 & 11 & 13.1 & 14 \\
\hline 34 Ethyl alcohol & 0.00442 & 24 & 0.00164 & 22 & 0.00221 & 16 & 0.00041 & 21 & 0.00596 & 24 \\
\hline 35 Ethyleimine & 2.93 & 13 & 1.48 & 5 & 0.727 & 9 & 0.15 & 17 & 11.4 & 15 \\
\hline 36 Ethylene dibromide & 0.209 & 27 & 0.465 & 6 & 0.0333 & 15 & 0.00268 & 32 & 1.5 & 30 \\
\hline 37 Ethylene thiourea & 0.1 & 11 & 0.0273 & 5 & 0.0145 & 6 & 0.00281 & 18 & 0.132 & 23 \\
\hline 38 Heptachlor & 0.18 & 3 & 0.00401 & 3 & Inadequate & $<3$ & 0.000482 & 8 & 0.214 & 6 \\
\hline 39 Hexachlorobenzene & 0.42 & 9 & 0.0169 & 6 & 0.0128 & 5 & 0.000575 & 10 & 0.149 & 3 \\
\hline 40 Hexachlorobutadiene & 0.15 & 7 & 0.334 & 7 & 0.0617 & 10 & 0.0267 & 14 & 1.45 & 13 \\
\hline 41 Hexachloroethane & 0.00304 & 3 & 0.00618 & 6 & 0.00147 & 3 & 0.000946 & 5 & 0.0349 & 6 \\
\hline 42 Hydrazine & 0.359 & 11 & 0.127 & 11 & 0.146 & 13 & 0.127 & 18 & 2.05 & 16 \\
\hline 43 Diphenythydrazine & 0.642 & 6 & Inadequate & $<3$ & 0.357 & 3 & 0.0146 & 8 & 0.485 & 9 \\
\hline 44 Hydrogen peroxide $(90 \%)$ & 0.549 & 16 & 5.02 & 6 & 0.11 & 10 & 0.0849 & 20 & 4.8 & 12 \\
\hline 45 Kepone & 0.729 & 4 & 0.15 & 7 & 0.118 & 3 & 0.00662 & 7 & 3.49 & 9 \\
\hline 46 Lindane & 0.4 & 9 & 0.338 & 9 & 0.2 & 9 & 0.0632 & 17 & 1.01 & 16 \\
\hline 47 3-Methylchlolanthrene & 1 & 69 & 1.25 & 3 & 0.175 & 17 & 0.245 & 38 & 3.32 & 36 \\
\hline 48 Methyl hydrazine & 0.0372 & 4 & 0.524 & 12 & 0.386 & 14 & 0.286 & 9 & 6.17 & 12 \\
\hline 49 Methyl methanesulfonate & 0.289 & 83 & 0.2 & 11 & 0.0606 & 37 & 0.0314 & 68 & 1.19 & 85 \\
\hline 50 MNNG (70-25-7) & 1.25 & 85 & 0.0894 & 9 & 0.348 & 34 & 0.281 & 74 & 2 & 59 \\
\hline 51 2-Naphthylamine & 0.3 & 35 & 1 & 5 & 0.04 & 15 & 0.0475 & 35 & 0.825 & 35 \\
\hline 52 Nitrogen mustard & 6.41 & 31 & 6 & 15 & 2.67 & 22 & 0.9 & 37 & 37.5 & 41 \\
\hline 53 Phenacetin & 0.0112 & 16 & 0.0109 & 7 & 0.0101 & 11 & 0.00109 & 19 & 0.0423 & 25 \\
\hline 54 Phenobarbital & 0.009 & 23 & 0.0109 & 7 & 0.0112 & 12 & 0.001 & 21 & 0.0312 & 26 \\
\hline 55 Reserpine & 8.5 & 10 & 0.733 & 16 & 0.524 & 15 & 0.245 & 12 & 9.33 & 26 \\
\hline
\end{tabular}


Table G.4 (continued)

\begin{tabular}{|c|c|c|c|c|c|c|c|c|c|c|}
\hline Test compound & $\begin{array}{l}\text { RP to } \\
\text { B(a)P }\end{array}$ & Matches & $\begin{array}{l}\mathrm{RP} \text { to } \\
\text { nicotine }\end{array}$ & Matches & $\begin{array}{l}\mathrm{RP} \text { to } \\
\text { cisplatin }\end{array}$ & Matches & $\begin{array}{l}\mathrm{RP} \text { to } \\
\text { aflatoxin }\end{array}$ & Matches & $\begin{array}{c}\text { RP to } \\
\text { cyclophosphamide }\end{array}$ & Matches \\
\hline 56 Safrole & 0.156 & 30 & 0.0207 & 8 & 0.012 & 15 & 0.00562 & 26 & 0.234 & 33 \\
\hline 57 Sodium saccharin & 0.593 & 13 & 0.011 & 6 & 0.00139 & 6 & 0.000845 & 17 & 0.0183 & 18 \\
\hline 58 2,3,7,8,-TCDD & 250 & 19 & 87.8 & 12 & 203 & 8 & 54.9 & 25 & 1000 & 27 \\
\hline 59 Tetrachloroethane & 0.0282 & 6 & 0.0385 & 4 & 0.0209 & 8 & 0.00329 & 6 & 0.19 & 10 \\
\hline 60 Tetrachloroethylene & & & 0.00227 & 5 & 0.0027 & 8 & 0.0012 & 6 & 0.0302 & \\
\hline 61 Thioacetamide & 0.226 & & 0.0433 & 4 & 0.0583 & 7 & 0.00208 & 26 & 0.128 & 2 \\
\hline 62 Toxaphene & 0.014 & 6 & 0.141 & 9 & 0.0892 & 8 & 0.02 & 15 & 0.807 & 15 \\
\hline 63 Trichloroethylene & 0.0434 & 17 & 0.00885 & 10 & 0.0127 & 11 & 0.00421 & 14 & 0.0651 & 23 \\
\hline 64 2,4,6-Trichlorophenol & 0.05 & 7 & 0.061 & 3 & 0.03 & 5 & 0.002 & 9 & 0.0833 & 10 \\
\hline 65 Urethane & 0.0625 & 48 & 0.0045 & 9 & 0.0103 & 20 & 0.00455 & 42 & 0.0421 & 43 \\
\hline 66 Vinyl chloride & 0.00547 & 4 & Inadequate & $<3$ & 0.0264 & 4 & 0.000174 & 10 & 0.261 & 17 \\
\hline 67 Vinylidene chloride & 0.0309 & 6 & 0.0172 & 3 & 0.0111 & 5 & 0.0107 & 6 & 0.5 & 11 \\
\hline
\end{tabular}


In Table G.4, each of the 67 compounds was compared to 5 different reference compounds as described earlier for the CRASH method. Thus, the scale of each column of relative potency values in Table G.4 is normalized to unity for the particular reference compound indicated in the column heading. Certain small inconsistencies may be noted for compounds found in a row and a column heading because the CRASH rules of matching are different for interviewing and reference compounds. The other noticeable pattem as described earlier is that greater dispersion is seen when $\mathrm{B}(\mathrm{a}) \mathrm{P}$ is one of the two reference compounds because of the absence of acute toxicity data for $\mathrm{B}(\mathrm{a}) \mathrm{P}$. Overall, it is demonstrated clearly from the panels in Fig. G.9, that, in principle, all of the reference compounds demonstrate the same pattern of relative potency estimates. The compound-specific scales in the RP columns of Table G.4 were all converted to a common scale based on B(a)P as shown in Table G.5. The conversions were based on factors of $1,3.2,8.2,8.08$, and 1 for B(a)P, nicotine, cisplatin, aflatoxin $B_{1}$, and cyclophosphamide, respectively, and in essence represents the potency of each of the other compounds relative to $B(a) P$.

The composite RP values based on five reference compounds shown in column 8 of Table G.5 were used to produce the scatter plots shown in Fig. G.10 for TD50 estimates in mice and rats and for the maximum dose tested (Max-D) in the NTP protocols of the Gold database. Generally only 50 animals of a particular sex and species were used and random error from that effect is seen in Fig. G.10. Again, in Fig. G.10 some of the erratic hormone action is observed with DES. In addition, hydrogen peroxide, bis(chloromethyl)ether, and bis-(2-chloroethyl)ether are frequent outliers on the scatter plots. As seen in Table G. 6 the plots are consistent to within a factor of about $\pm 11 X$ for the TD50 and $\pm 7.32 \mathrm{X}$ for the maximum doses tested based on $\pm \sigma$ which envelopes about $68 \%$ of the comparisons. The $\mathrm{TD}_{50}$ is larger, perhaps due to the condition that the $\mathrm{TD}_{50}$ values were dependent on the collection of dose-response models used for this particular list of 67 compounds and because some dose-response extrapolation was required between the experimental doses tested and the $\mathrm{TD}_{50}$. In principle, extrapolation to higher doses is required for some compounds and to lower doses for others.

Considering the variability commonly associated with toxicological data, it seems remarkable that $68 \%$ of the estimates fall within an order of magnitude of the relative-potency adjusted median value for both the $\mathrm{TD}_{50}$ and the maximum experimental dose tested as seen in Fig. G.11. This order-ofmagnitude accuracy is remarkable given the level of variability usually associated with various bioassay results for a particular compound. The next section will demonstrate how the relative potency for a particular compound can be used to estimate future regulatory guidelines or risk coefficients depending on whether the compound becomes considered as a carcinogenic or non-carcinogenic substance.

\section{G.5.4 Prediction of Regulations or Permissible Exposures Based on RP}

\section{G.5.4.1 Reference Dose}

Jones, Owen, Trabalka, Bamthouse, and Easterly (1991) used RASH-based relative potency values and EPA reference dose (RfD) values for mercury, lead, toluene, pentachlorophenol, cresol, phenol, and barium to estimate permissible near-term concentrations in drinking water for currently unregulated compounds. These seven substances were selected based on the consideration that each has been used extensively and major regulatory changes are not expected unless a substance becomes reclassified as a carcinogen, neurotoxin, or teratogen with respect to human health. A regression equation was 

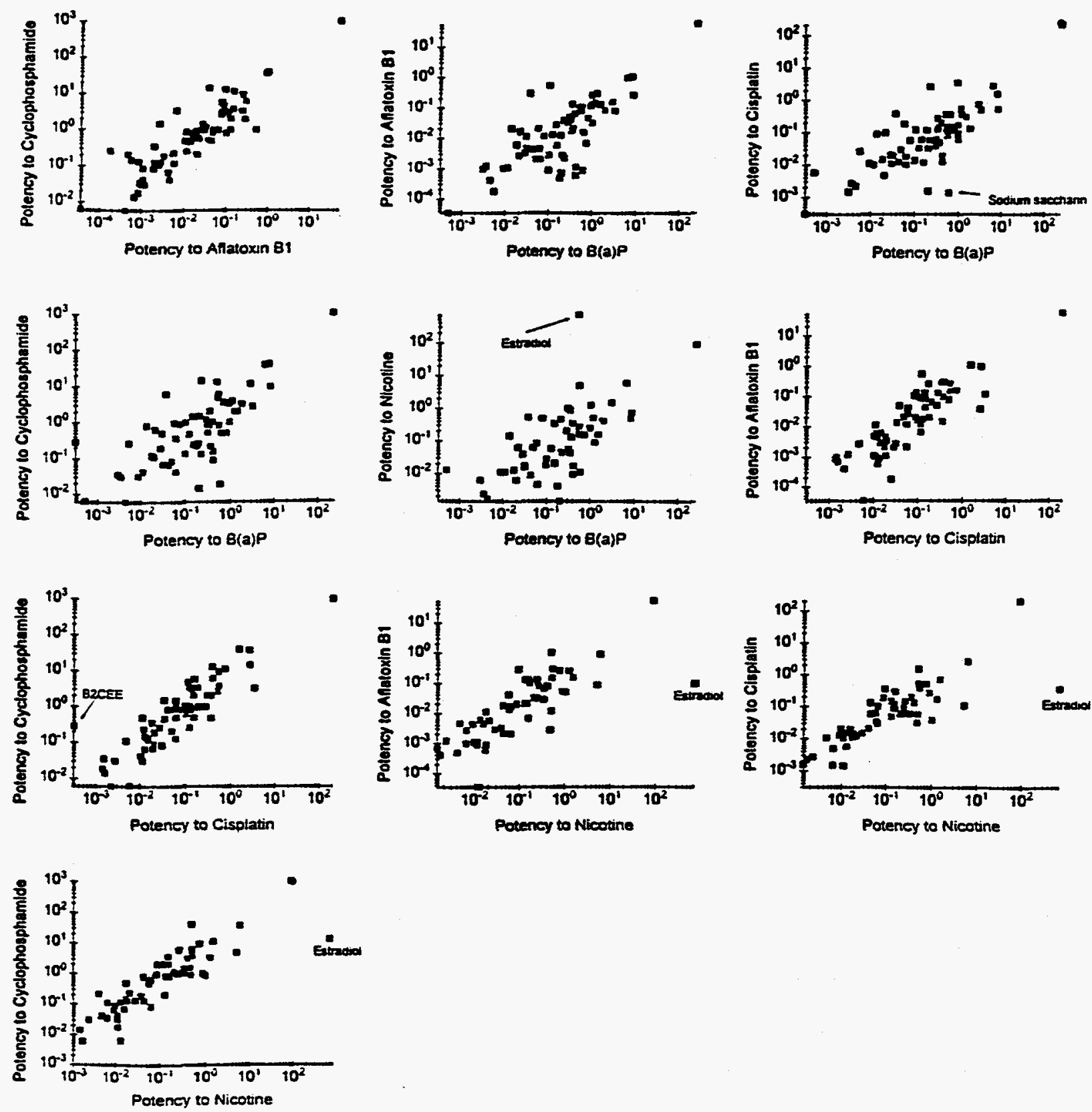

Fig. G.9. Scatter plots of the potency of 67 compounds relative to 5 different reference compounds taken two at a time. 
Table G.5. Common scales of toxicological potency for 67 compounds based on RPs to five reference compounds from Table G.4

Conversion to a common scale having unit potency for $B(a) P$ was achieved by factors of $3.2,8.2,8.08$, and 1.0

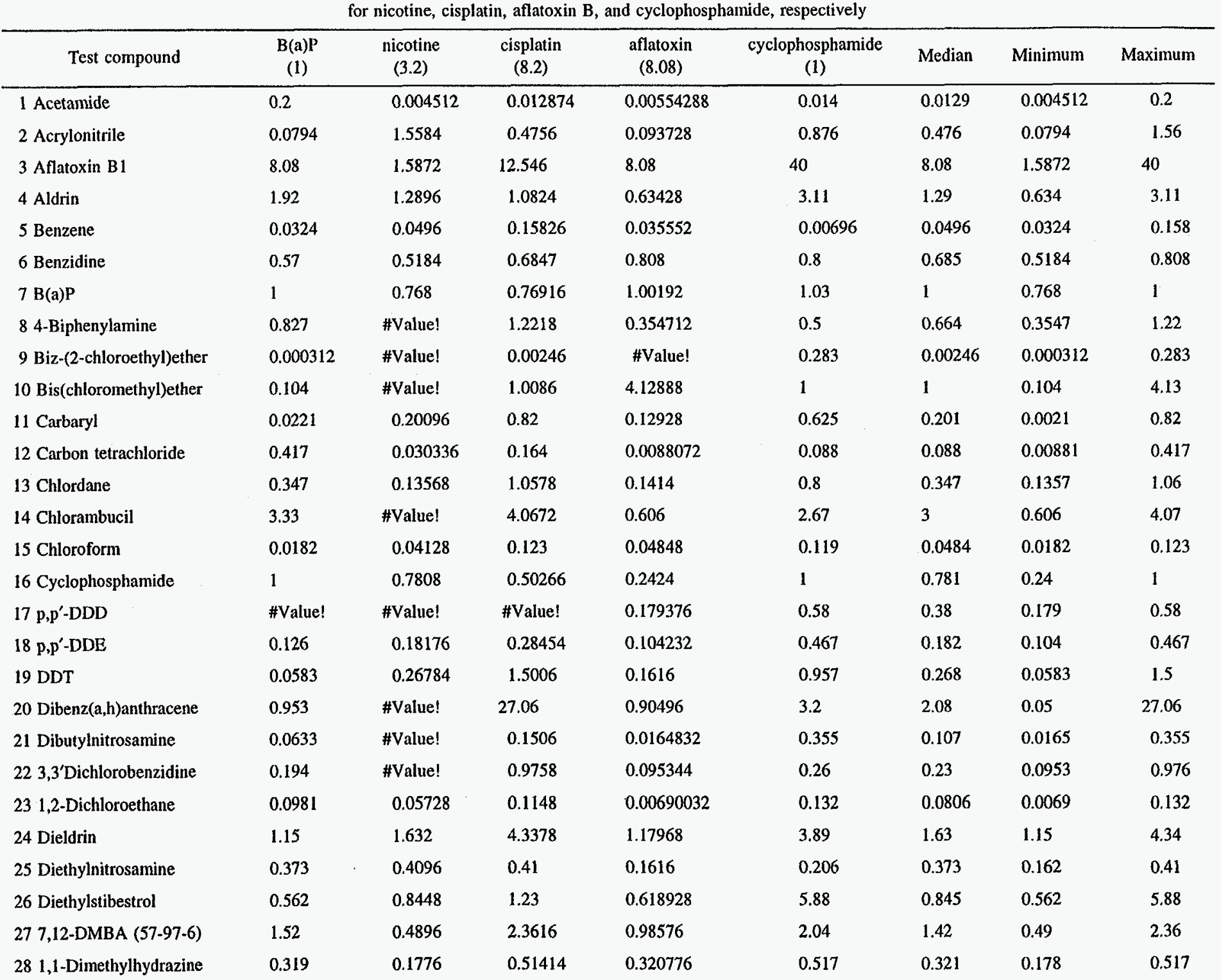


Table G.5 (continued)

\begin{tabular}{|c|c|c|c|c|c|c|c|c|}
\hline Test compound & $\begin{array}{l}B(a) P \\
\text { (1) }\end{array}$ & $\begin{array}{l}\text { nicotine } \\
(3.2)\end{array}$ & $\begin{array}{l}\text { cisplatin } \\
\text { (8.2) }\end{array}$ & $\begin{array}{l}\text { aflatoxin } \\
(8.08)\end{array}$ & $\begin{array}{l}\text { cyclophosphamide } \\
\text { (1) }\end{array}$ & Median & Minimum & Maximum \\
\hline 29 Dimethylnitrosamine & 0.34 & 2.7584 & 2.214 & 0,402384 & 0.995 & 0.995 & 0.34 & 2.76 \\
\hline 31 Diphenylnitrosamine & 0.0202 & 0.02016 & 0.039032 & 0.0212504 & 0.11 & 0.02125 & 0.02016 & 0.11 \\
\hline 32 ENNG (4245-77-6) & 0.227 & \#Value! & 21.156 & 0.303 & 14.4 & 7.35 & 0.227 & 21.16 \\
\hline 35 Ethyleimine & 2.93 & 4.736 & 5.9614 & 1.212 & 11.4 & 4.74 & 1.21 & 11.4 \\
\hline 36 Ethylene dibromide & 0.209 & 1.488 & 0.27306 & 0.0216544 & 1.5 & 0.273 & 0.0217 & 1.5 \\
\hline 37 Ethylene thiourea & 0.1 & 0.08736 & 0.1189 & 0.0227048 & 0.132 & 0.0874 & 0.0227 & 0.132 \\
\hline 38 Heptachlor & 0.18 & 0.012832 & \#Value! & 0.00389456 & 0.214 & 0.0964 & 0.00389 & 0.214 \\
\hline 42 Hydrazine & 0.359 & 0.4064 & 1.1972 & 1.02616 & 2.05 & 1.03 & 0.359 & 2.05 \\
\hline 43 Diphenythydrazine & 0.642 & \#Value! & 2.9274 & 0.117968 & 0.485 & 0.564 & 0.118 & 2.93 \\
\hline 44 Hydrogen peroxide (90\%) & 0.549 & 16.064 & 0.902 & 0.685992 & 4.8 & 0.902 & 0.549 & 16.06 \\
\hline 45 Kepone & 0.729 & 0.48 & 0.9676 & 0.0534896 & 3.49 & 0.729 & 0.0535 & 3.49 \\
\hline 46 Lindane & 0.4 & 1.0816 & 1.64 & 0.510656 & 1.01 & 1.01 & 0.4 & 1.64 \\
\hline 47 3-Methylchlolanthrene & 1 & 4 & 1.435 & 1.9766 & 3.32 & 1.98 & 1 & 4 \\
\hline 48 Methyl hydrazine & 0.0372 & 1.6768 & 3.1652 & 2.31088 & 6.17 & 2.31 & 0.0372 & 6.17 \\
\hline 49 Methyl methanesulfonate & 0.289 & 0.64 & 0.49692 & 0.253712 & 1.19 & 0.497 & 0.25 & 1.19 \\
\hline 50 MNNG $(70-25-7)$ & 1.25 & 0.28608 & 2.8536 & 2.37048 & 2 & 2 & 0.286 & 2.85 \\
\hline
\end{tabular}


Table G.5 (continued)

\begin{tabular}{|c|c|c|c|c|c|c|c|c|}
\hline Test compound & $\begin{array}{l}\mathrm{B}(\mathrm{a}) \mathrm{P} \\
(1)\end{array}$ & $\begin{array}{c}\text { nicotine } \\
(3.2)\end{array}$ & $\begin{array}{l}\text { cisplatin } \\
(8.2)\end{array}$ & $\begin{array}{l}\text { aflatoxin } \\
(8.08)\end{array}$ & $\begin{array}{c}\text { cyclophosphamide } \\
\text { (1) }\end{array}$ & Median & Minimum & Maximum \\
\hline 56 Safrole & 0.156 & 0.06624 & 0.0984 & 0.0454096 & 0.234 & 0.0984 & 0.0454 & 0.234 \\
\hline 58 2,3,7,8,-TCDD & 250 & 280.96 & 1664.4 & 443.592 & 1000 & 444 & 250 & 1665 \\
\hline 59 Tetrachloroethane & 0.0282 & 0.1232 & 0.17138 & 0.0265832 & 0.19 & 0.123 & 0.0266 & 0.19 \\
\hline 62 Toxaphene & 0.014 & 0.4512 & 0.73144 & 0.1616 & 0.807 & 0.451 & 0.014 & 0.807 \\
\hline 63 Trichloroethylene & 0.0434 & 0.02832 & 0.10414 & 0.0340168 & 0.0651 & 0.0434 & 0.02832 & 0.104 \\
\hline 64 2,4,6-Trichlorophenol & 0.05 & 0.1952 & 0.246 & 0.01616 & 0.0833 & 0.0833 & 0.01616 & 0.246 \\
\hline 65 Urethane & 0.0625 & 0.0144 & 0.08446 & 0.036764 & 0.0421 & 0.0421 & 0.0144 & 0.0845 \\
\hline
\end{tabular}

$\underset{\infty}{\stackrel{\Xi}{\sharp}}$ 

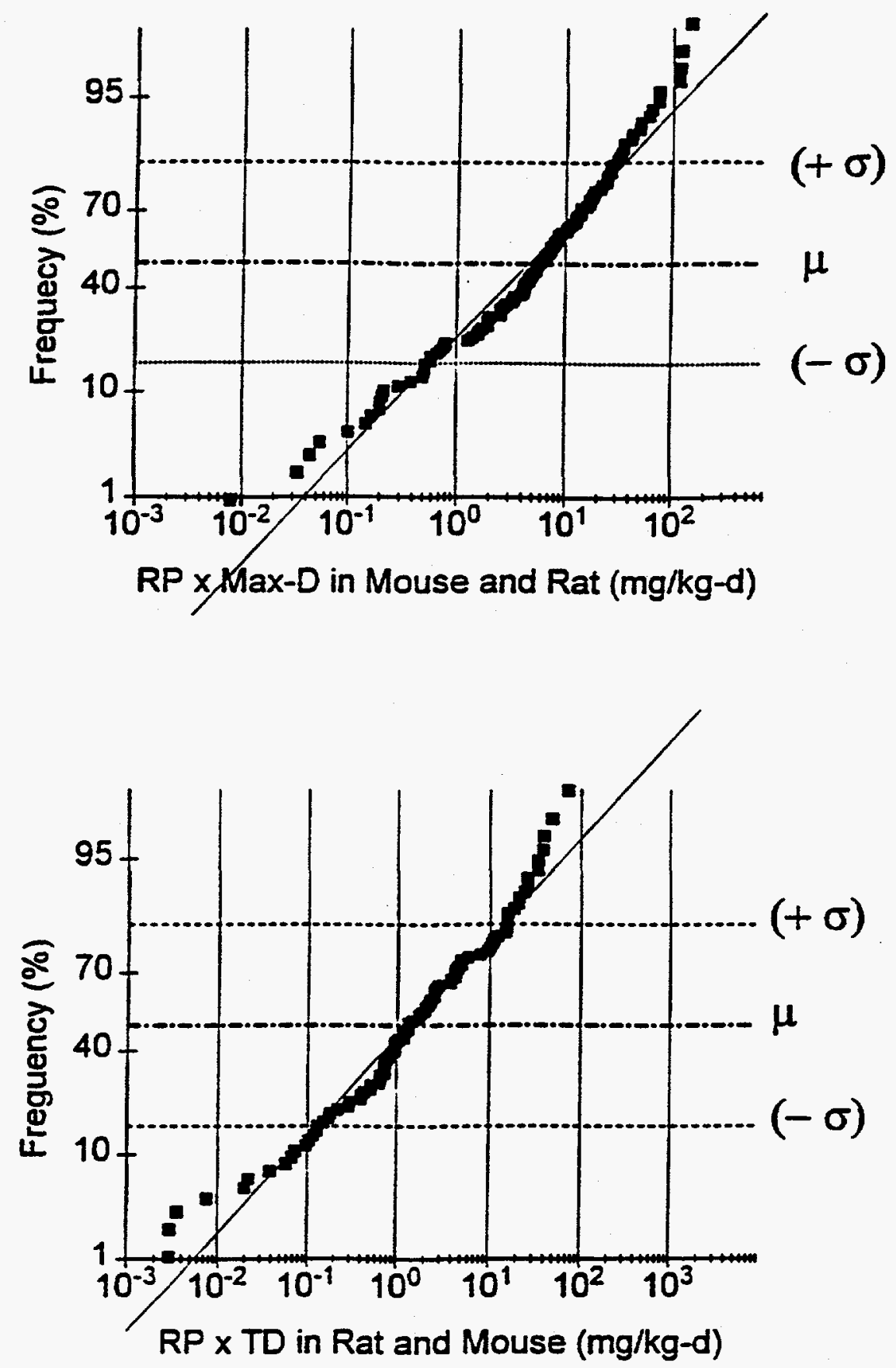

Fig. G.10. Median relative potency values from Table G.5 were compared with the $\mathbf{T D}_{50}$ estimates from Gold et al. (1989) and the maximum doses tested in the 2-year rodent carcinogenesis tests. 
Table G.6. Fitted values for a log-normal frequency distribution function comparing potency-adjusted $\mathbf{T D}_{50}$ and maximum doses tested for rats, mice, and mice plus rats The "untransformed" values are based on a unit relative potency for $\mathrm{B}(\mathrm{a}) \mathrm{P}$

\begin{tabular}{lcccc}
\hline \multicolumn{1}{c}{ Data fitted } & Log 10 mean & Log 10 sigma & $\begin{array}{c}\text { Untransformed } \\
\text { mean }\end{array}$ & $\begin{array}{c}\text { Untransformed } \\
\text { sigma }\end{array}$ \\
\hline RP $\times$ TD50 (mouse) & 0.299 & 0.972 & $2.00 \mathrm{mg} / \mathrm{kg} / \mathrm{d}$ & Factor of 9.38 \\
$\mathrm{RP} \times$ TD50 (rat) & 0.0848 & 1.06 & $1.22 \mathrm{mg} / \mathrm{kg} / \mathrm{d}$ & Factor of 11.5 \\
$\mathrm{RP} \times$ TD50 (both) & 0.158 & 1.04 & $1.44 \mathrm{mg} / \mathrm{kg} / \mathrm{d}$ & Factor of 11.0 \\
$\mathrm{RP} \times$ Max-D (mouse) & 0.84 & 0.877 & $6.92 \mathrm{mg} / \mathrm{kg} / \mathrm{d}$ & Factor of 7.50 \\
$\mathrm{RP} \times$ Max-D (rat) & 0.499 & 0.822 & $3.16 \mathrm{mg} / \mathrm{kg} / \mathrm{d}$ & Factor of 6.63 \\
$\mathrm{RP} \times$ Max-D (both) & 0.617 & 0.865 & $4.75 \mathrm{mg} / \mathrm{kg} / \mathrm{d}$ & Factor of 7.32 \\
\hline
\end{tabular}

developed to estimate permissible concentration for any new compound $i$ in drinking water from that new compound's $\mathrm{RP}_{\mathrm{i}}$ on the scale normalized to unity for $\mathrm{B}(\mathrm{a}) \mathrm{P}$ :

$$
\text { Concentration }_{\mathbf{i}}(\mathrm{mg} / \mathrm{L})=(0.022 \mathrm{mg} / \mathrm{L}) / \mathrm{RP}_{\mathrm{i}} \text {. }
$$

\section{G.5.4.2 Carcinogenic Risk Coefficients}

Similarly, Jones, Owen, Trabalka, Barnthouse, and Easterly (1991) used risk coefficients derived by the EPA Carcinogen Assessment Group (CAG) to estimate risk for a compound which might eventually be reclassified as a carcinogen. Risk coefficients for known human carcinogens beryllium oxide, chromium VI, acrylonitrile, nickel subsulfide, coke oven emissions, nickel refinery dust, arsenic, benzene, cadmium, and benzidine were based on epidemiological data and were selected because they do not seem to include the inflated upper-limits found in slope factors (i.e., risk coefficients) based on animal experiments. Slope factors were adjusted to account for oral intake. From an analysis of RP and slope for the ten substances, an estimate of risk based on $R P_{i}$ and dose $D_{i}$ becomes

$$
\text { Risk }=4.7 \times R P_{i}(\mathrm{mg} / \mathrm{kg}-\mathrm{d})^{-1} \times D_{i}(\mathrm{mg} / \mathrm{kg}-\mathrm{d})
$$

\section{G.5.5 Common Scale for Radiological and Toxicological Hazards}

Dose units between radiological and chemical hazards are quite distinct in spite of earlier agency goals of developing a generalized method for chemical dosimetry. Due to lack of progress with respect to generalized chemical dosimetry based on fundamental considerations such as structure-activity relationships or ionic configuration, most comparisons are currently reduced to the exercise of comparing insult levels that elicit a particular level of response in a selected bioassay. This brings us exactly back to the recommendations of Committee 17 of the Environmental Mutagen Society that were made before the venture into generalized chemical dosimetry. 

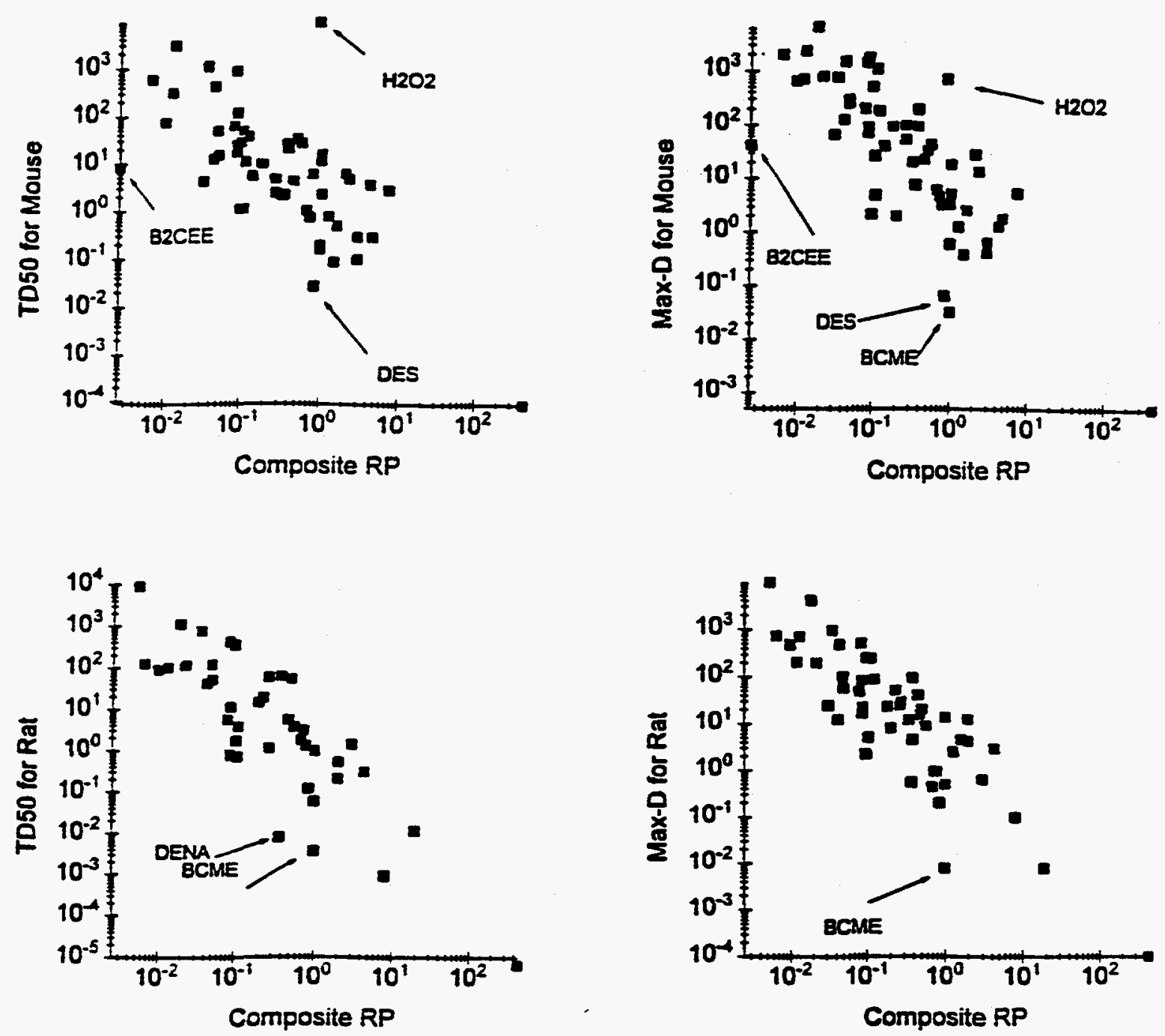

Fig. G.11. Cumulative log-normal frequency plots for potency-adjusted to $\mathrm{TD}_{50}$ doses (Gold et al. 1989) and maximum doses tested (Gold et al. 1984).

\section{G.5.6 Common Scale for Radiological and Toxicological Hazards}

The common-scale approach seems to be best served by comparing the radiation dose of exposure with the chemical dose counterpart-both transported to human organ tissues of risk-for one or more index response levels where comparisons are possible-hopefully, where effects of mathematical interpolation and extrapolation models are minimized. The index response level should be chosen based on the available test data for the selected bioassay used to bridge from radiological to chemical effects. Then the respective doses, both radiological and chemical, associated with the common response can be compared with their respective hypothetical human exposure counterparts. The algebra, in this case, helps to clarify the words as seen in Table G.3.

A strategy for direct comparison of risk from radiological exposures relative to risk from an alternative chemical exposure can be demonstrated as shown in Table G.3. If $R$ is used as the effective dose of 
concern from depleted uranium and $\mathrm{C}$ is the chemical dose of concern from tungsten, then these two insults are expected to have differential degrees of toxicity/risk/hazard and that differential effect can be estimated directly from the classical hazard index formulation. The typical hazard index for either radiological or chemical applications involves comparison of an exposure or dose of concern (numerator of a ratio) to a regulation, guideline, or reference exposure expressed in the same units (denominator of the ratio). The denominator of the two hazard index quantities should be associated with equal levels of response in the same bioassay. Thus, a natural common scale is established between radiological and toxicological exposures. Because the hazard index ratio is without dose units (i.e., $\mathrm{R} / \mathrm{ri}$ for radiation and $\mathrm{C} / \mathrm{ci}$ for chemicals, where ri is identical to $\mathrm{ci}$ in the bioassay of choice) a hazard index for depleted uranium can be compared directly with its counterpart for tungsten-at least in theory.

Radiation comparisons, in general, are readily established because the dosimetry concepts apply to all ionizing radiations of different linear energy transfer (LET) or ionic stopping power qualities. Furthermore, radiation has been tested in various bioassays such as those listed in the RTECS for assessments of chemical toxicity. Table G.7 lists radiation dose-response values usable as the denominators of various hazard indices.

For chemical compounds, as with radiation, it is only required that the hazard index be unitless. Therefore, for in vitro tests the insult may be expressed in concentration as with $\mathrm{mg} / \mathrm{L}$ or in the form of Haber's dose based on the product of concentration and exposure time. Similarly, for dose to in vivo targets, the insult can be expressed as $\mathrm{mg}$ of insult per $\mathrm{kg}$ of body weight or alternatively as the integral of organ concentration as a function of time, over time. Either can be used, and the choice may depend upon the available data and whether realistic or upper limit estimates are required. Doseresponse data for bioassays that can be compared to the radiological assays described in Table G.7 can be taken from the RTECS, the published literature, or new laboratory data taken when the published data are inadequate to answer the questions of concern.

\section{G.5.7 Relative Potency Values for Tungsten and Its Compounds Compared with Sodium Chromate}

The following comparisons will be based on the same five reference compounds that were used to demonstrate relative potency relations to the $\mathrm{TD}_{50}$ and the maximum dose tested in the NTP data base as prepared by Gold et al. (1989 and 1984), viz., B(a)P, nicotine, cisplatin, aflatoxin, and cyclophosphamide. Because few bioassay results are available for tungsten compounds, it seemed desirable to select a demonstration compound for comparison purposes that has been tested more extensively and, as a metal, has some implications regarding the toxicity of tungsten.

Because chromium is also in Class VI-B of the periodic table, sodium chromate $\left(\mathrm{CrO}_{4} \cdot 2 \mathrm{Na}\right.$; $\mathrm{CAS} \#$ 7775-11-3) was used since it has been tested in a variety of bioassays. In addition to demonstrating how the relative potency array is generated for sodium chromate, it provides data that could be used to create several denominator values required for the numerical bridges associated with the hazard index scales for radiological and toxicological hazards.

The bioassay data available from RTECS for sodium chromate are listed in Table G.8. The test systems include a number of short-term in vitro bioassays as well as $\mathrm{LD}_{50}$ and $L D$ Lo data from acute toxicity studies in vivo in several mammalian species. The in vitro bioassays include, among others, tests for mutation in microorganisms, DNA damage and repair, gene conversion and mitotic 
Table G.7. Bioassay-specific radiation dose-response values for the denominator of the hazard index formulation

30 Day Lethality to Humans or Test Animals:

$\mathrm{LD}_{90}=4 / 3 \times \mathrm{LD}_{50}$;

$\mathrm{LD}_{50}=290 \mathrm{cGy}$ [Humans-for animals see publications of Morris and Jones for species, sex, strain, radiation, dose, rate, and time of protraction forces on the $\mathrm{LD}_{50}$. The data base is enormous but is readily available to the experienced user via the ORNL report by Jones et al. (1986);

$\mathrm{LD}_{10}=2 / 3 \times \mathrm{LD}_{50}$ (Jones, Morris, and Young 1994).

\section{Cancer Risk:}

Risk $=0.00071 \times$ Dose $(\mathrm{cGy})($ UNSCEAR 1988).

\section{Occupational Limit:}

5 Rem/yr.

\section{Natural Background:}

Terrestrial $=28 \mathrm{mrem} / \mathrm{yr} ;$ Cosmic $=27 \mathrm{mrem} / \mathrm{yr}$; Internal $=39 \mathrm{mrem} / \mathrm{yr}$

Radon $=2.4 \mathrm{rem} / \mathrm{yr}$

(NAS Biological Effects of Ionizing Radiations

BEIR V, 1990).

\section{Mammalian Cell Survival:}

Hela: $S=1-[1-\exp \{\text { Dose } / 128\}]^{2}$

Human Embryo: $S=1-[1-\exp \{\text { Dose } / 221\}]^{2}$

Hamster: $S=1-[1-\exp \{\text { Dose/132 }\}]^{3.2}$

Rabbit: $S=1-[1-\exp \{\text { Dose } / 125\}]^{1}$

Mouse: $S=1-[1-\exp \{\text { Dose } / 115\}]^{2}$

Mouse Lymphoma: $S=1-[1-\exp \{\text { Dose/114 }\}]^{1}$

Mouse Marrow/Spleen: $S=1-[1-$

$\exp \left\{\right.$ Dose/115\}] ${ }^{2}$ (Casarett 1968).

\section{Mammalian Cell Mutation:}

Yield $\left(6 \mathrm{TG} / 10^{6}\right.$ cells $)=0.04+6.0 \times$ Dose $(\mathrm{cGy})$

(TK-6 human diploid lymphoblasts; Grosovsky and Little 1985).
Chromosome Aberrations in Mammalian Cells: Acute Exposure:

Yield $($ per cell $)=0.0476 \times$ Dose $(\mathrm{cGy})+(6.19 \times$ $\left.10^{-4}\right) \times \operatorname{Dose}^{2}$ (cGy) (Human lymphocytes; Lloyd et al. 1975).

Acute Exposure:

Yield $($ per cell $)=0.01+\left(1.9 \times 10^{-4}\right) \times$ Dose $(\mathrm{cGy})$ $+\left(1.8 \times 10^{-6}\right) \times$ Dose $^{2}$ (cGy) (CH Liver Cells;

Brooks et al. 1971).

Chronic Exposure:

Yield $($ per cell $)=0.02+\left(3.3 \times 10^{-4}\right) \times$ Dose $(\mathrm{cGy})$ (CH Liver Cells; Brooks et al. 1971).

SCEs in Mammalian Cells:

Yield (per mitosis) $=5.50=(0.050) \times$ Dose $(\mathrm{cGy})$ $\left(\mathrm{G}_{1}\right.$ of Human Lymphocytes; Abramovsky et al. 1978).

Yield (per mitosis) $=5.60=(0.0075) \times$ Dose $(\mathrm{cGy})$ $\left(\mathrm{G}_{2}\right.$ of Human Lymphocytes; Abramovsky et al. 1978).

Micronuclei in Mammalian Cells:

Yield (per cell) $=\left(1.17 \times 10^{-3}\right) \times$ Dose $(\mathrm{cGy})+(8.7$ $\left.\times 10^{-7}\right) \times \operatorname{Dose}^{2}(\mathrm{cGy})+0.0129$

(Human lymphocytes; Prosser et al. 1988).

Yield (per cell) $=\left(1.39 \times 10^{-3}\right) \times$ Dose $(\mathrm{cGy})+$ $\left(3.68 \times 10^{-6}\right) \times \operatorname{Dose}^{2}(\mathrm{cGy})+0.0113$

(Human lymphocytes; Kormos and Koteles 1988).

Mutations in Drosophila Sperm:

Yield $($ per cell $)=0.002+\left(2.24 \times 10^{-5}\right) \times$ Dose

(cGy)

(Spenser and Stern 1948).

Cytotoxicity of E. Coli:

Yield $=\left(3\right.$ to $\left.5 \times 10^{-5}\right) \mathrm{cGy}^{-1}$;

(Diver 1982; Karran 1980; Purdy et al. 1979)

Mutations in E. Coli:

Yield $=\left(4 \times 10^{-10}\right)$ mutants/(survivor-cGy)

(Demerec 1985) 
Table G.8. Toxicological test data for sodium chromate (VI) [RTECS \#GB2955000]

\begin{tabular}{|c|c|c|c|c|c|c|}
\hline RTECS No. & $\begin{array}{c}\text { Test } \\
\text { class }^{a}\end{array}$ & Test type & Dosage & Units & Timing & Sex \\
\hline GB2955000 & 1 & dnr-sat & 50 & $\mathrm{mmol} / \mathrm{L}$ & & \\
\hline GB2955000 & 1 & mmo-esc & 50 & $\mathrm{nmol} / \mathrm{L}$ & & \\
\hline GB2955000 & 1 & dnr-bcs & 5 & $\mathrm{mmol} / \mathrm{L}$ & & \\
\hline GB2955000 & 1 & mmo-smc & 25 & $\mathrm{mmol} / \mathrm{L}$ & & \\
\hline GB2955000 & 1 & mrc-smc & 25 & $\mathrm{mmol} / \mathrm{L}$ & & \\
\hline GB2955000 & 1 & dnd-ham-lng & 200 & $\mu \mathrm{mol} / \mathrm{L}$ & $2 \mathrm{H} \mathrm{C}$ & \\
\hline GB2955000 & 1 & cyt-ham-lng & 2500 & $\mathrm{nmol} / \mathrm{L}$ & & \\
\hline GB2955000 & 1 & cyt-ham-ovr & 250 & $\mu \mathrm{g} / \mathrm{L}$ & & \\
\hline GB2955000 & 1 & sce-ham-ovr & 250 & $\mu \mathrm{g} / \mathrm{L}$ & & \\
\hline GB2955000 & 1 & sce-ham-lng & 32 & $\mu \mathrm{g} / \mathrm{L}$ & & \\
\hline GB2955000 & 1 & msc-ham-lng & 2500 & $\mathrm{nmol} / \mathrm{L}$ & & \\
\hline GB2955000 & 1 & dnd-ckn-emb & 2500 & $\mathrm{nmol} / \mathrm{L}$ & & \\
\hline GB2955000 & 2 & ipr-rat TDLo & 5 & $\mathrm{mg} / \mathrm{kg}$ & $5 \mathrm{D}$ & male \\
\hline GB2955000 & 4 & irp-rat LD50 & 57 & $\mathrm{mg} / \mathrm{kg}$ & & \\
\hline GB2955000 & 4 & inv-dog LDLo & 235 & $\mathrm{mg} / \mathrm{kg}$ & & \\
\hline GB2955000 & 4 & inv-cat LD50 & 164 & $\mathrm{mg} / \mathrm{kg}$ & & \\
\hline GB2955000 & 4 & scu-rbt LDLo & 243 & $\mathrm{mg} / \mathrm{kg}$ & & \\
\hline GB2955000 & 4 & inv-rbt LDLo & 32 & $\mathrm{mg} / \mathrm{kg}$ & & \\
\hline GB2955000 & 4 & idr-rbt LDLo & 250 & $\mathrm{mg} / \mathrm{kg}$ & & \\
\hline GB2955000 & 4 & skn-gpg LDLo & 206 & $\mathrm{mg} / \mathrm{kg}$ & & \\
\hline GB2955000 & 4 & ipr-gpg LDLo & 206 & $\mathrm{mg} / \mathrm{kg}$ & & \\
\hline GB2955000 & 4 & scu-gpg LDLo & 30 & $\mathrm{mg} / \mathrm{kg}$ & & \\
\hline GB2955000 & 4 & idr-gpg LDLo & 382 & $\mathrm{mg} / \mathrm{kg}$ & & \\
\hline GB2955000 & 4 & ipr-mus LD50 & 32 & $\mathrm{mg} / \mathrm{kg}$ & & \\
\hline
\end{tabular}

${ }^{a} \mathrm{O}=$ irritation; 1 = mutation; 2 = reproduction; 3 = tumorigenic; $4=$ acute toxicity.

recombination, and chromosome effects. Based on these data, relative potency values were generated for sodium chromate relative to $\mathrm{B}(\mathrm{a}) \mathrm{P}$, cyclophosphamide, nicotine, aflatoxin $\mathrm{B}_{1}$, and cisplatin, converted to a common scale based on $\mathrm{B}(\mathrm{a}) \mathrm{P}$ for which $\mathrm{RP}=1$, and are displayed in Table G.9.

The limited bioassay data for tungsten and three of its compounds as obtained from RTECS are presented in Table G.10. Nearly all of the values derive from acute toxicity testing $\left(\mathrm{LD}_{50}, \mathrm{LD}_{0}, \mathrm{TD}_{\mathrm{Lo}}\right)$ of a few mammalian species by various routes of administration. The very sparse information from in vitro bioassays includes one microbial mutation value, two data points from cytogenetic studies, a few gene conversion and mitotic recombination values and two from phage inhibition capacity assays. 
Table G.9. Summary of the potency of four tungsten compounds and sodium chromate relative to $B(a) P$, cyclophosphomide, nicotine, aflatoxin $B_{1}$, and cisplatin all converted to a common scale indexed by unit potency for $B(a) P$; cyclophosphomide, nicotine, aflatoxin, and cisplatin were standardized according to potencies listed at the bottom of the table

\begin{tabular}{lcccccccc}
\hline \multicolumn{1}{c}{ Test compound } & CAS \# & RTECS \# & B(a)P & Cycloph & Nicot & Aflatox & Cisp & Med \\
\hline Tungsten & $7440-33-7$ & Y07175000 & 1700 & 0.052 & 0.0093 & 0.017 & 0.01 & 0.017 \\
Matches & & & 1 & 2 & 1 & 2 & 1 & \\
Tungsten oxide & $1314-35-8$ & Y07760000 & ND & 0.088 & 0.088 & 0.022 & 0.12 & 0.088 \\
Matches & & & 0 & 2 & 2 & 2 & 2 & \\
Sodium tungstate & $13472-45-2$ & Y07875000 & 0.034 & 0.018 & 0.059 & 0.012 & 0.31 & 0.034 \\
Matches & & & 3 & 5 & 7 & 6 & 4 & \\
Tungstic acid, S.S.D. & $10213-10-2$ & Y07900000 & 0.16 & 0.48 & 0.18 & 0.24 & 0.27 & 0.24 \\
Matches & & & 2 & 2 & 2 & 3 & 3 & \\
& & & & & & & & \\
Sodium chromate (VI) & $7775-11-3$ & GB2955000 & 0.33 & 0.17 & 17 & 17 & 16 & 16 \\
Matches & & & 8 & 8 & 7 & 8 & 7 & \\
B(a)P & $50-32-8$ & DJ3675000 & 1 & 1 & 3.2 & 8.08 & 8.2 & \\
Matches & & & Self & 73 & 8 & 74 & 46 & \\
\hline
\end{tabular}


Table G.10. Bioassay test data for tungsten [YO7175000], tungsten oxides [YO7760000], sodium tungstate [YO7875000], and tungstic acid, sodium salt, dihydrate [YO7900000]

\begin{tabular}{|c|c|c|c|c|c|c|}
\hline RTECS No. & $\begin{array}{c}\text { Test } \\
\text { class }^{a}\end{array}$ & Test type & Dosage & Units & Timing & Sex \\
\hline YO7175000 & 4 & ipr-rat LD50 & 5000 & $\mathrm{mg} / \mathrm{kg}$ & & \\
\hline YO7175000 & 4 & inh-gpg TD & 150 & $\mathrm{mg}$ & & \\
\hline YO7175000 & 4 & inh-gpg TD & 250 & $\mathrm{mg} / \mathrm{kg}$ & & \\
\hline YO7175000 & 4 & unr-rat LD50 & 2000 & $\mathrm{mg} / \mathrm{kg}$ & & \\
\hline YO7175000 & 2 & orl-ckn TDLo & 0.61 & $\mathrm{mg} / \mathrm{kg}$ & & $150 \mathrm{D}$ pre \\
\hline YO7175000 & 2 & orl-rat TDLo & 1.16 & $\mathrm{mg} / \mathrm{kg}$ & & $30 \mathrm{~W}$ pre/1-20D preg \\
\hline YO7175000 & 2 & orl-rat TDLo & 1.21 & $\mathrm{mg} / \mathrm{kg}$ & & $35 \mathrm{~W}$ pre \\
\hline YO7175000 & 0 & skn-rbt & 500 & $\mathrm{mg}$ & 24H MLD & \\
\hline YO7175000 & 0 & eye-rbt & 500 & $\mathrm{mg}$ & $24 \mathrm{H} \mathrm{MLD}$ & \\
\hline YO7760000 & 4 & orl-rat LD50 & 6417 & $\mathrm{mg} / \mathrm{kg}$ & & \\
\hline YO7760000 & 4 & inh-rat TD & 250 & $\mathrm{mg} / \mathrm{kg}$ & & \\
\hline YO7760000 & 4 & orl-rat TD & 840 & $\mathrm{mg} / \mathrm{kg}$ & & \\
\hline YO7760000 & 4 & orl-rat LD50 & 1059 & $\mathrm{mg} / \mathrm{kg}$ & & \\
\hline YO7875000 & 4 & ims-rat SD50 & 221 & $\mathrm{mg} / \mathrm{kg}$ & & \\
\hline YO7875000 & 4 & ims-rbt LD50 & 105 & $\mathrm{mg} / \mathrm{kg}$ & & \\
\hline YO7875000 & 4 & inv-cat LD50 & 139 & $\mathrm{mg} / \mathrm{kg}$ & & \\
\hline YO7875000 & 4 & orl-ckn LD & 650 & $\mathrm{mg} / \mathrm{kg}$ & & \\
\hline YO7875000 & 4 & orl-gpg LD0 & 500 & $\mathrm{mg} / \mathrm{kg}$ & & \\
\hline YO7875000 & 4 & orl-gpg LD50 & 1152 & $\mathrm{mg} / \mathrm{kg}$ & & \\
\hline YO7875000 & 4 & orl-mus LD50 & 240 & $\mathrm{mg} / \mathrm{kg}$ & & \\
\hline YO7875000 & 4 & orl-rat LD0 & 700 & $\mathrm{mg} / \mathrm{kg}$ & & \\
\hline YO7875000 & 4 & orl-rat LD50 & 1190 & $\mathrm{mg} / \mathrm{kg}$ & & \\
\hline YO7875000 & 4 & orl-rat LD50 & 8840 & $\mathrm{mg} / \mathrm{kg}$ & & \\
\hline YO7875000 & 4 & orl-rbt LD0 & 500 & $\mathrm{mg} / \mathrm{kg}$ & & \\
\hline YO7875000 & 4 & orl-rbt LD50 & 875 & $\mathrm{mg} / \mathrm{kg}$ & & \\
\hline YO7875000 & 4 & scu-dog LDLo & 140 & $\mathrm{mg} / \mathrm{kg}$ & & \\
\hline YO7875000 & 4 & scu-gpg LDLo & 810 & $\mathrm{mg} / \mathrm{kg}$ & & \\
\hline YO7875000 & 4 & scu-rat LD50 & 240 & $\mathrm{mg} / \mathrm{kg}$ & & \\
\hline YO7875000 & 4 & scu-rbt LDLo & 78 & $\mathrm{mg} / \mathrm{kg}$ & & \\
\hline YO7875000 & 2 & inh-rat TCLo & 504 & $\mu \mathrm{g} / \mathrm{m}^{3}$ & $24 \mathrm{H}$ & $17 \mathrm{~W}$ male \\
\hline YO7875000 & 1 & pic-esc & 5 & $\mathrm{mmol} / \mathrm{L}$ & & \\
\hline YO7875000 & 1 & mrc-dmg-orl & $1.00 \mathrm{E}+00$ & $\mathrm{mmol} / \mathrm{L}$ & & \\
\hline
\end{tabular}


Table G.10 (continued)

\begin{tabular}{lclllll}
\hline RTECS No. & $\begin{array}{c}\text { Test } \\
\text { class }^{a}\end{array}$ & \multicolumn{1}{c}{ Test type } & Dosage & Units & Timing & Sex \\
\hline YO7875000 & 1 & mrc-smc & 100 & $\mathrm{mmol} / \mathrm{L}$ & \\
YO7875000 & 1 & mmo-smc & 100 & $\mathrm{mmol} / \mathrm{L}$ & \\
YO7875000 & 1 & sin-smc & 100 & $\mathrm{mmol} / \mathrm{L}$ & \\
YO7875000 & 1 & mrc-ham-emb & 0.1 & $\mathrm{mg} / \mathrm{ml}$ & \\
YO7900000 & 4 & ipr-mus LD0 & 99 & $\mathrm{mg} / \mathrm{kg}$ & \\
YO7900000 & 4 & ipr-mus LD50 & 135 & $\mathrm{mg} / \mathrm{kg}$ & \\
YO7900000 & 4 & ipr-rat LD50 & 204 & $\mathrm{mg} / \mathrm{kg}$ & \\
YO7900000 & 4 & ipr-rat LD0 & 66 & $\mathrm{mg} / \mathrm{kg}$ & \\
YO7900000 & 4 & orl-gpg LD & 1304 & $\mathrm{mg} / \mathrm{kg}$ & \\
YO7900000 & 4 & orl-gpg LD & 791 & $\mathrm{mg} / \mathrm{kg}$ & \\
YO7900000 & 4 & orl-gpg LD & 781 & $\mathrm{mg} / \mathrm{kg}$ & \\
YO7900000 & 1 & cyt-hmn-lym & 10 & $\mathrm{mg} / \mathrm{L}$ & \\
YO7900000 & 1 & pic-esc & 412 & $\mu \mathrm{g} / \mathrm{well}$ & \\
YO7900000 & 1 & cyt-hmn-lym & 30 & $\mathrm{mg} / \mathrm{L}$ & $\mathrm{g} / \mathrm{kg}$ \\
YO7900000 & 2 & inv-mus-TDLo & 330 & $\mathrm{preg}$ \\
\hline
\end{tabular}

${ }^{a} \mathrm{O}=$ irritation; 1 = mutation; 2 = reproduction; 3 = tumorigenic; 4 = acute toxicity.

Most of these in vitro data are for the more soluble sodium tungstate, a few for tungstic acid, and none for tungsten or tungsten oxide.

Given this small data base, the number of usable matches with data on the five reference compounds was quite small, but sufficient to calculate relative potency values and then convert to a common scale using the potency of $\mathrm{B}(\mathrm{a}) \mathrm{P}=1.0$ as described previously. The relative potency values for tungsten and its compounds are shown in Table G.9; note the general similarity of potencies for tungsten, tungsten oxide, and sodium tungstate against most of the reference compounds and in the common scale despite the limited matches in assay data on which to base calculations. The relative potency data for sodium tungstate based on the five reference compounds are plotted individually in Fig. G.12; a combined plot is also shown for which all comparisons are put on the common scale with the potency of $\mathrm{B}(\mathrm{a}) \mathrm{P}=1.0$. From this combined figure, the very limited available data suggest that tungsten, if it were a carcinogen, would have a potency of about 0.04 relative to $\mathrm{B}(\mathrm{a}) \mathrm{P}$ with one standard deviation having a range of 0.004 to 0.4 . Because the data are so limited, this estimate of potency may not be as reliable as those obtained for other substances where useable data were far more plentiful.

For comparison, the relative potency of sodium tungstate was compared directly to that of sodium chromate (VI) using data from five bioassays in common between them. The results are illustrated in Fig. G.13. The median potency of sodium tungstate is about $20 \%$ that of sodium chromate in this analysis, a concordance that gives some confidence in the results obtained for the tungsten compounds despite the uncertainty inherent in the limited number and types of assay data on which to base potency calculations. 


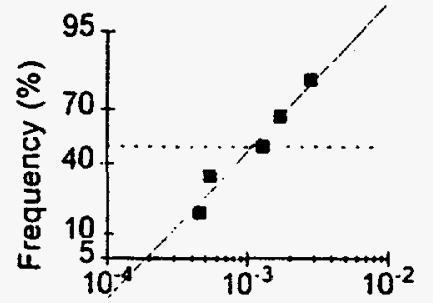

Sodium Tungstate to Aflatoxin B1

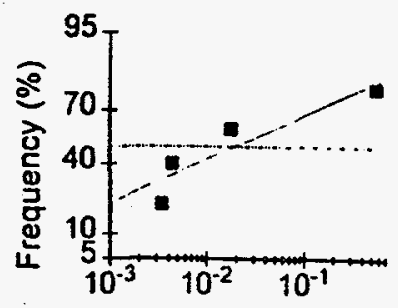

Sodium Tungstate to Cyclophosphamide

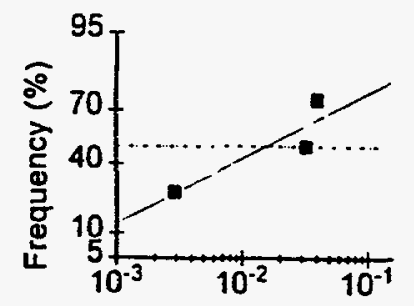

Sodium Tungstate to Cisplatin
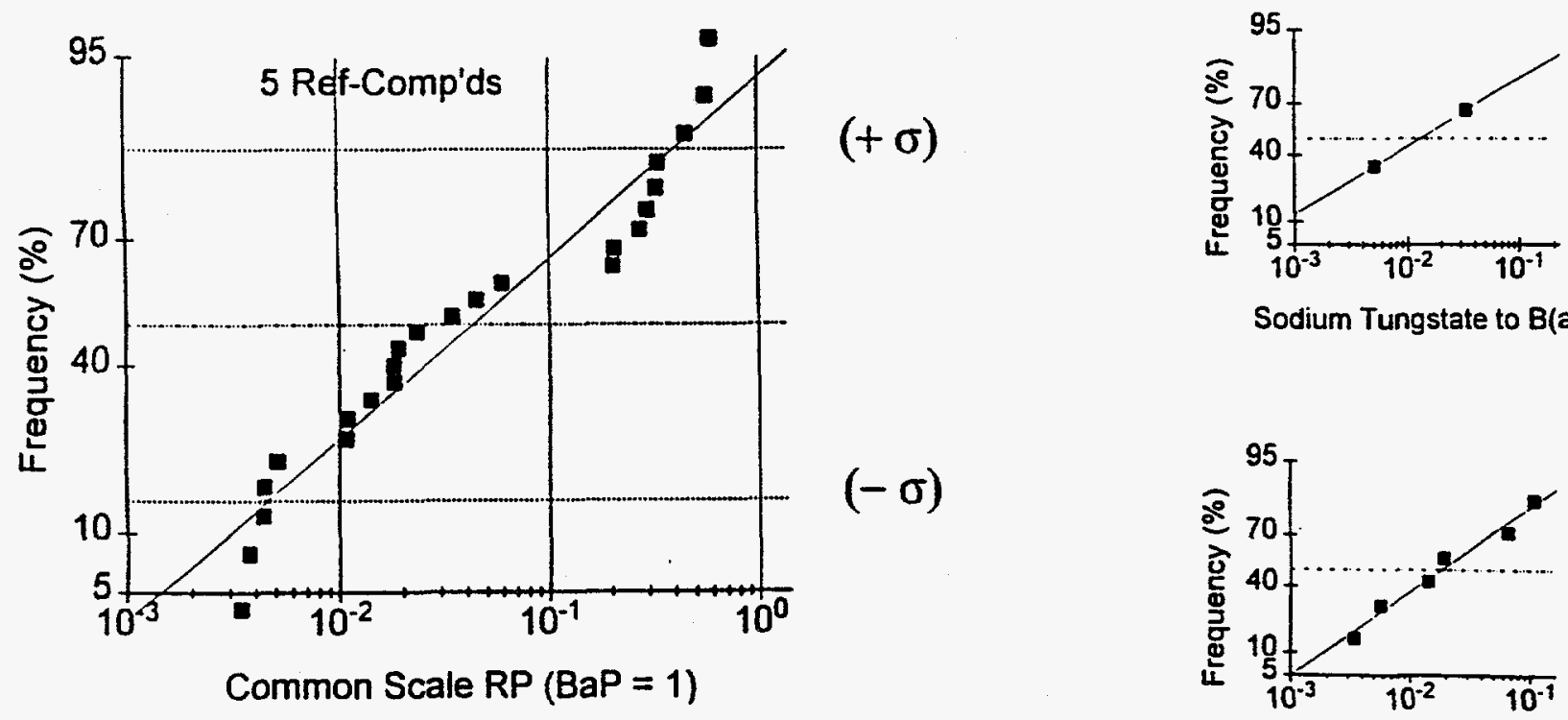

Sodium Tungstate to B(a)P

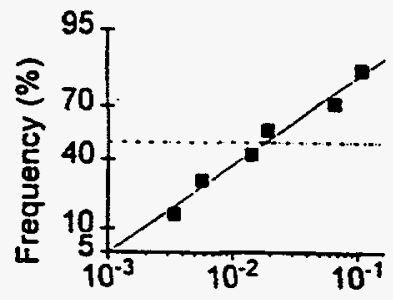

Sodium Tungstate to Nicotine

Fig. G.12. Graphical comparisons of potencies completed in Table G.9.

As described earlier, the potential risk of cancer for a compound of potency $R P_{i}$ relative to $B(a) P$ can be estimated by:

$$
\text { Risk }=\left(4.7 \times \mathrm{RP}_{\mathrm{i} / \mathrm{B}(\mathrm{a}) \mathrm{P}}\right) \times \text { Dose }(\mathrm{mg} / \mathrm{kg} \text {-day })(\text { Jones } 1995)
$$

This risk factor is based on data normalized to $\mathrm{B}(\mathrm{a}) \mathrm{P}$ and assumes a lifetime (70-year) oral exposure because it is derived in part from EPA slope factors for ten human carcinogens identified through epidemiological analyses. From the above relationship and the potency of 0.04 for tungsten compared to $\mathrm{B}(\mathrm{a}) \mathrm{P}$, one can calculate the lifetime ingestion rate or dose associated with any chosen level of risk. For example, to maintain a lifetime risk from continuous exposure at or below the $1 \times 10^{-4}$ level, the ingestion rate of tungsten would need to be at or below:

$$
\begin{gathered}
\text { Dose }_{\mathrm{w}}=1 \times 10^{-4} /[4.7 \times 0.04]=1.9 \times 10^{-3} \mathrm{mg} /\{\mathrm{kg}-\text { day }\} \\
\text { Dose }_{\mathrm{w}}=1.9 \mathrm{~g} /\{\mathrm{kg} \text {-day }\}
\end{gathered}
$$




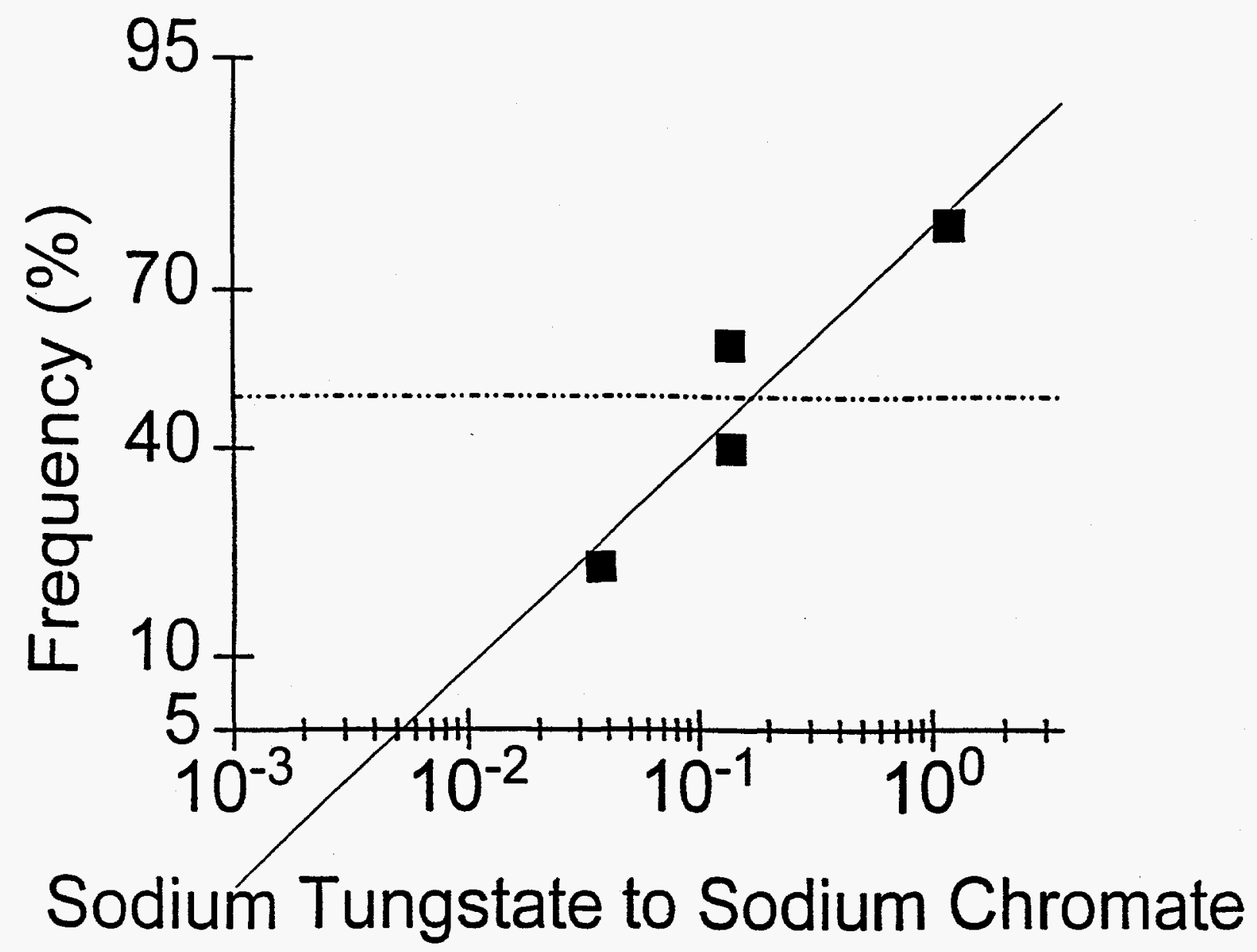

Fig. G.13. From the few common bioassays for sodium tungstate and sodium chromate (VI), it appears that the median potency of sodium tungstate is about $20 \%$ that of sodium chromate.

Again, it should be stressed that tungsten and its compounds have not been identified as carcinogens nor has carcinogenicity been ruled out; the only animal data are equivocal and no human data are available. Should tungsten eventually be found to be carcinogenic, this preliminary analysis of estimated potency leads to the above ingestion rate corresponding to the $1 \times 10^{-4}$ cancer risk level.

\section{G.6 TOXICOLOGICAL DATA NEEDED FOR TUNGSTEN}

From the current philosophy that compensatory cell proliferation is associated with all stages of carcinogenesis as reviewed earlier, and that most other bioassays directly address mutagenic potential for alterations in the DNA of a cell or demonstrate through clastogenic action that a compound has left its signature in the tissues at risk, the available bioassay results cannot readily be dismissed as irrelevant to potential hazards from tungsten. As noted earlier, the observation by Ecobichon (1992) that "A basic tenet of toxicology is that the more frequent a specific adverse health effect can be 
measured in different animal species, the greater is the likelihood that, at some dosage, a similar effect might occur in the human."

For both of these reasons which may not necessarily be distinct, it is highly desirable to have a wide diversity of bioassay results for both radiological and toxicological hazards. From such an array of independent estimates of the hazard index, one can easily compare the mean values and variances of the log-normal distributions. One can also evaluate whether the bioassays or data considered directly relevant to carcinogenesis are supported by the other bioassays relevant to initiation or promotion. It is far more informative to compare frequency distributions than to implement the common approach of comparing point estimates that may have been badly distorted by the use of assumptions and linear extrapolation models.

The available data base for tungsten and its compounds is quite limited as seen in Table G.10, particularly with regard to short-term bioassays. The low solubility of most forms of tungsten may limit the compounds that can be tested in vitro, but additional assays could certainly be done using the more soluble forms such as sodium tungstate. It would be desirable to pick assays to match available data for the reference compounds when possible, but it would also be valuable to obtain data concurrently on tungsten and the five reference compounds for measures such as cell proliferation, cytotoxicity, cell turnover, and other relevant endpoints for which no data are already extant. 


\section{G.7 REFERENCES}

Abramovsky, I; Vorsanger, G; and Hirschhorn K. Sister-chromatid exchange induced by X-ray of human lymphocytes and the effect of L-cysteine. Mut. Res. 50:93-100, 1978.

American Conference of Government Industrial Hygienists. Threshold Limit Values and Biological Exposure Indices for 1989-1990. 6500 Glenway Ave. Bldg. D-7. Cincinnati, OH 45211-4438.

Ames, B. N. Natural carcinogens; they're found in many foods. Health \& Environment 4:4-6, 1990.

Bergonie, J. and Tribondeau, L. Interpretation de quelques resultats de la radiotherapie et essai de fixation de'une technique rationalle. C. R. Acad. Sci. Paris, v. 143, p. 982, 1906. (Translation on p. 3 of V. P. Bond, Fliedner, T. M., and Archambeau, J. O. 1965. Mammalian radiation lethality. Academic, New York.

Brooks, A. L.; Mead, D. K.; and Peters, R. F. Effect of chronic exposure to ${ }^{60} \mathrm{Co}$ on the frequency of metaphase chromosome aberrations in the liver cells of the Chinese hamster (in vivo). Int. J. Radiat. Biol 20:599-604, 1971.

Brooks, A. L.; Peters, R. F.; and Rollag, M. D. Metaphase chromosome aberrations in Chinese hamster liver cells in vivo after single acute ${ }^{60}$ Co exposure. Rad. Res. 45:191-201, 1971.

Casarett, A. P. Radiation Biology. Prentice-Hall, Inc. Englewood Cliffs, New Jersey, 1968.

Chemical Industry Institute of Toxicology. CIIT Hosts Cell Proliferation Workshop. CIIT Activities 9(11-12), 1989.

Committee 17 of the Environmental Mutagen Society. Environmental Mutagenic Hazards. Science 187:503-514, 1975.

Conolly, R. B. U.S. EPA Reassessment of the Health Risks of 2,3,7,8-Tetrachlorodibenzo-p-dioxin (TCDD). CIIT Activities 14(1-8), 1994.

Demerec, M. Induced mutations and possible mechanisms of the transmission of heredity in Escherichia coli. Proc. Natl. Acad. Sci. USA. 21:27-37, 1985.

Department of Defense: Office of the Secretary: 1987. Defense Priority Model (Environmental). Federal Register 52:44204-44206.

Department of Defense: Office of the Secretary. 1989. Defense Priority Model;Defense Environmental Restoration Program. Federal Register 54:43104-43106.

Diver, W. P.; Sargentini, N. J. Mutation in Escherichia coli that selectively sensitizes cells grown in rich medium to X or UV radiation, or methanesulfonate. Int. J. Radiat Biol 42:339-346, 1982.

Ecobichon, D. J. The Basis of Toxicity Testing. Chemical Rubber Corporation Press, Boca Raton, 1992. 
Emerit, I. Clastogenic factors, a link between chronic inflammation and carcinogenesis. p. 59 in P. A. Cerutti, O. F. Nygaard, M. C. Simic (eds.) Anticarcinogenesis and Radiation Protection. Plenum Press. New York, 1987.

Finney, D. J. Probit Analysis. Cambridge University Press, 1971.

Gold, L. S.; Sawyer, C. B.; Magaw, R.; Backman, G. M.; de Veciana, M.; Levinson, R.; Hooper, N. K.; Havender, W. R.; Bernstein, L.; Peto, R.; Pike, M. C.; and Ames, B. N. A carcinogenic potency database of the standardized result of animal bioassays. Environ Health Persp 58:9-319, 1984.

Gold, L. S.; Slone, T. H.; Bernstein, L. Summary of carcinogenic potency and positivity for 492 rodent carcinogens in the carcinogenic potency database. Environ Health Persp 79:259-272, 1989.

Gunnison, A. F.; Dulak, L.; Chiang, G.; Zaccardi, J.; and Farruggella. A sulphite-oxidase-deficient rat model: Subchronic toxicology. Fd Cosmet Toxicol 19:221-232, 1981.

Iball, J. The relative potency of carcinogenic compounds. The Am. J. of Cancer. 35:188-190, 1939.

International Agency for Research on Cancer. Scientific Report 116 on Mechanisms of Carcinogenesis in Risk Assessments. Lyon, 1992.

International Programme on Chemical Safety: Environmental Health Criteria 109. Summary Report of the Evaluation of Short-Term Tests for Carcinogens (Collaborative Study on In Vivo Tests). World Health Organization. Geneva, 1990.

Jones, T. D. A Unifying Concept for Carcinogenic Risk Assessments: Comparison with RadiationInduced Leukemia in Mice and Men. Health Physics. 47:533-558, 1984.

Jones, T. D. 1991. (Invited Editorial) On the Future of Regulating Chemicals. Environmental Auditor 2:101-102.

Jones, T. D. 1993. Hazardous Substances, Regulation by Government Agencies. Chapter in Handbook of Hazardous Materials, Academic Press, Inc. pp. 321-331.

Jones, T. D. Use of bioassays in assessing health hazards from complex mixtures: A RASH analysis. Chemosphere 31:2475-2484, 1995.

Jones, T. D.; Griffin, G. D.; and Walsh P. J. A Unifying Concept for Carcinogenic Risk Assessments. J. Theoret. Biol. 105:35-61, 1983.

Jones, T. D.; Jacobs, D. J.; Auxier, J. A.; and Kerr G. D. 1979. Risk of Cancer Based on Promotion from Cytotoxicity. National Bureau of Standards Special Publication 554, p. 41, Washington D.C. U.S. Department of Commerce. 
Jones, T. D.; Morris, M. D.; Wells, S. M.; Young, R. W. Animal mortality resulting from uniform exposures to photon radiations: Calculated $\mathrm{LD}_{50} \mathrm{~s}$ and a compilation of experimental data. Oak Ridge National Laboratory Report ORNL 6338, 1986.

Jones, T. D.; Owen, B. A.; and Trabalka, J. R. Protection of Human Health from Mixtures of Radionuclides and Chemicals in Drinking Water. Arch of Environ Contam and Toxicol 20:143-150, 1991.

Jones, T. D.; Owen, B. A.; Trabalka, J. R.; Barnthouse, L. W.; Easterly, C. E. 1991. Chemical Pollutants: A Caricaturized Logos for Future Planning. Environmental Auditor 2:71-88.

Jones, T. D.; Walsh, P. J.; Watson, A. P.; Owen, B. A.; Barnthouse, L. W.; and Sanders, D. A. 1988.

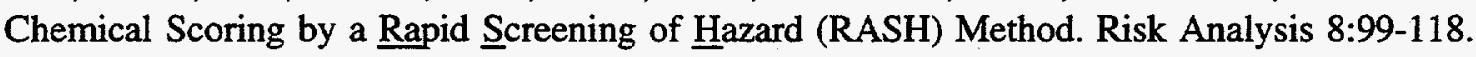

Jones, T. D.; Walsh, P. J.; and Zeighami, E. A. 1985. Permissible Concentrations of Chemicals in Air and Water Derived from RTECS Entries: A "RASH" Chemical Scoring System. Toxicol. Indust. Health 1:213-234.

Karran, P.; Lindahl, T., Oefsteng, I., and Evensen, G. B. Eschericia coli mutants deficient in 3-methyladenine-DNA glycosylase. J. Mol. Biol. 140:101-127, 1980.

Kouri, R. E.; Henry, C. J.; and Kreisher, J. H. Stages in Carcinogenesis. Preface figure in R. E. Kouir (ed.) Genetic Differences in Chemical Carcinogenesis. CRC Press, Inc., Boca Raton, Florida, 1980.

Kormos, C. and Koteles, G. J. Micronuclei in X-irradiated human lymphocytes. Mut. Res. 199:31-35, 1988.

Lloyd, D. C.; Purrott, R. J.; Dolphin, G. W.; Bolton, D.; Edwards, A. A.; and Corp, M. J. The relationship between chromosome aberrations and low-LET radiation dose to human lymphocytes. Int. J. Radiat. Biol. 28:75, 1975.

Morris, M. D. and Jones, T. D. Hematopoietic death of unprotected man from photon radiations: statistical modeling from animal experiments. Int. J. Radiat. Biol. 55:445-461, 1989.

National Council on Radiation Protection and Measurements. NCRP Report No. 96 on Comparative Carcinogenicity of Ionizing Radiation and Chemicals. 7910 Woodmont Ave. Bethesda, MD 20814.

Prosser, J. S.; Moquet, J. E.; Lloyd, D. C.; and Edwards, A. A. Radiation induction of micronuclei in human lymphocytes. Mut. Res. 199:37-45, 1988.

Purdy, M. A.; Yielding, K. L. Enhancement of post-ultraviolet killing in Escherichia coli $k-12$ through the action of gyrase inhibitors: Evidence for associated gyrase-RECBC deoxyribonuclease function. Antimicrobial Agents Chemother. 16:247-251, 1979.

RASH 19XX. Jones, T. D. and Basavaraju BPCSR. 1995; Jones, T. D. 1995. Toxicological Potency of 2,3,7,8-Tetrachlorodibenzo-p-dioxin relative to 100 other compounds: A relative potency 
Analysis of in vitro and in vivo test data. Arch of Environ Contam and Toxicol 29:77-85; Jones, T. D. 1995. Use of bioassays in assessing health hazards from complex mixtures: A RASH analysis. Chemosphere 31:2475-2484; Glass, L. R. and Easterly, C. E. 1994. Evaluating the tumorigenic potential of polynuclear aromatic hydrocarbons. Environ. Int. 20:475-481; Hileman, B. 1993. 'Expert intuition' tops in test of carcinogenic prediction. C\&EN June 21:35-37; Easterly, C. E., Jones, T. D., Glass, L. R., and Walsh, P. J. 1993. Biotesting wastewater for hazard evaluation. Water Res. 27:1145-1152; Jones, T. D. 1993. Hazardous Substances, Regulation by Government Agencies. Chapter in Handbook of Hazardous Materials, Academic Press, Inc. pp. 321-331; Watson, A. P.; Jones, T. D.; Adams, J. D. 1992. Relative potency estimates of acceptable residues and reentry intervals after nerve agent release. Ecotox. and Environ. Safety. 23:328-342; Glass, L. R.; Easterly, C. E.; Jones, T. D.; and Walsh, P. J. 1991. Ranking of carcinogenic potency using a relative potency approach. Arch. of Environ. Contam. and Toxicol. 21:169-176; Glass, L. R.; Jones, T. D.; Easterly, C. E.; Walsh, P. J. 1991. Use of Short-Term Test for the Prediction of the Hazard Represented by Potential Chemical Carcinogens. Environ. Auditor 2:103-169; Jones, T. D. 1991. (Invited Editorial) On the Future of Regulating Chemicals. Environmental Auditor 2:101-102; Jones, T. D. and Easterly, C. E. 1991. On the Rodent Bioassays Currently being Conducted on 44 Chemicals: A Rash Analysis to Predict Test Results from the National Toxicology Program. Mutagenesis 6:507-514; Jones, T. D.; Owen, B. A.; Trabalka, J. R. 1991. Protection of Human Health from Mixtures of Radionuclides and Chemicals in Drinking Water. Arch. Environ. Contam. Toxicol. 20:143-150; Jones, T. D.; Owen, B. A.; Trabalka, J. R.; Barnthouse, L. W.; Easterly, C. E. 1991. Chemical Pollutants: A Caricaturized Logos for Future Planning. Environmental Auditor 2:71-88; Owen, B. A. and Jones, T. D. 1990. Hazard Evaluation for Complex Mixtures: Relative Comparisons to Improve Regulatory Consistency. Regul. Toxicol. Pharm. 11:132-148; Watson, A. P.; Jones, T. D.; and Griffin, G. D. 1989. Sulfur mustard as a carcinogen: application of relative potency analysis to the chemical warfare agents $H, H D$, and HT. Regul. Toxicol. and Pharmacol. 10:1-25; Jones, T. D.; Walsh, P. J.; Watson, A. P.; Owen B. A.; Barnthouse, L. W.; and Sanders, D. A. 1988. Chemical Scoring by a Rapid Screening of Hazard (RASH) Method. Risk Analysis 8:99-118; Barr, J. T. 1985. The calculation and use of carcinogenic potency: A review. Regul Toxicol and Pharmacol 5:432-459; Jones, T. D.; Walsh P. J.; and Zeighami, E. A. 1985. Permissible Concentrations of Chemicals in Air and Water Derived from RTECS Entries: A "RASH" Chemical Scoring System. Toxicol. Indust. Health 1:213-234; Jones, T. D.; Griffin, G. D.; and Walsh, P. J. A Unifying Concept for Carcinogenic Risk Assessments. J. Theoret. Biol. 105:35-61, 1983.

Sax, N. I. 1979. Dangerous Properties of Industrial Materials, 5th Edition. van Nostrand Reinhold Co., New York.

Spenser, W. P. and Stern, C. Experiments to Test the Validity of the Linear R-Dose Mutation Frequency in Drosophila at Low Dosage. Genetics, 33:43-74, 1948.

Trosko, J. E. and Chang, C-c. An Integrative Hypothesis Linking Cancer, Diabetes and Atherosclerosis: The Role of Mutations and Epigenetic Changes. Medical Hypothesis $6: 455-468,1980$.

U.S. food and Drug Administration. Chapter I-Food and Drug Administration. Department of Health and Human Services (parts 170-199). Federal Register 36:20546, 1985. 


\section{INTERNAL DISTRIBUTION}

1. Central Research Library

2. Document Reference Section

3-4. ORNL Laboratory Records

5. ORNL Laboratory Records-RC

6. ORNL Patent Office

7-8. Metals and Ceramics Records Office

9. ORNL Site Manager

10. C. F. Baes

11. E. L. Bird

12. J. H. Clift

13. R. H. Cooper

14. G. E. Courville

15. D. F. Craig

16. R. M. Davis

17. C. Easterly

18. K. F. Eckerman

19. S. G. Frykman
20. H. W. Hayden

21. S. G. Hildebrand

22. T. D. Jones

23-28. C. R. Kerley

29. R. W. Leggett

30. R. A. Lowden

31-36. G. Mackiewicz-Ludtka

37. C. I. Moser

38. N. B. Munro

39. W. G. Northcutt

40. R. B. Ogle

41. E. K. Ohriner

42. R. M. Reed

43. R. B. Shelton

44. V. K. Sikka

45. M. L. Socolof

\section{EXTERNAL DISTRIBUTION}

46. AFRRI, Defense Nuclear Agency, 8901 Wisconsin Avenue, Bethesda, MD 20889-5603

E. P. Kearsley

47. CENTERS FOR DISEASE CONTROL AND PREVENTION, 4770 Buford Highway, MS F18, Atlanta, GA 30341-3724

D. Paschal

48. CORNING, INC., Technical Services and Administration, SP-FR-02-1, Sullivan Park, Corning, NY 14831

E. L. Menger

49. DOW CORNING CORPORATION, Safety and Environmental Affairs, Mail \#115, 3901 Saginaw Road, Midland, MI 48686-0995

H.W. Foglesong

50. ECONOMIC RESOURCE GROUP, INC., One Mifflin Place, Cambridge, MA 02138

Susan F. Tierney 
51. EGLIN AIR FORCE BASE, ATTN: WL/MNSE, 101 W. Eglin Boulevard, Eglin Air Force Base, FL 32542-6810

Don Harrison

52. U.S. ARMY ENVIRONMENTAL POLICY INSTITUTE, GEORGIA INSTITUTE OF TECHNOLOGY, 430 Tenth Street NW, Suite S-206, Atlanta, GA 30318-5768

E. W. Novak

53. GEORGIA INSTITUTE OF TECHNOLOGY, School of Civil and Environmental Engineering, Atlanta, GA 30332-0355

R. A. Gregory

54. LAW COMPANIES GROUP, INC., 114 Townpark Drive, Suite 250, Kennesaw, GA 30144-5599

G. F. Sowers

55. OFFICE OF NAVAL RESEARCH-EUROPE, 223/231 Old Marylebone Road, London, England NW1 STH

J. G. Simon

56-57. OFFICE OF SCIENTIFIC AND TECHNICAL INFORMATION, U.S.

Department of Energy, P.O. Box 62, Oak Ridge, TN 37831-0062

58. PEER CONSULTANTS, P.C., 1000 Ashley Drive, Suite 312, Tampa, FL 33602

L. A. Abron

59. PENNSYLVANIA STATE UNIVERSITY, Department of Materials Science and Engineering, 118 Steidle Building, University Park, PA 16802

K. E. Spear

60. U.S. ARMY ARMAMENT ENGINEERING DIRECTORATE, Picatinny Arsenal, NJ 07806

R. Bladja

61. U.S. ARMY ARMAMENT RESEARCH, DEVELOPMENT, AND

ENGINEERING CENTER, Environmental Technology Office (Pollution Prevention), ATTN: AM-STA-AR-ET, Building 172, Picatinny Arsenal, NJ 07806-5000

J. W. Frankovic 
62-75. U.S. ARMY ARMAMENT RESEARCH, DEVELOPMENT AND

ENGINEERING CENTER, Tank-Automotive and Armaments Command, Picatinny Arsenal, 07806-5000

ATTN: AMSTA-AR-IMC

AMSTA-AR-GLC

AMSTA-AR-AET, B355, William Ebihara

AMSTA-AR-CCH-C, B65, Michael Danesi

AMSTA-AR-AET-M, B1609, Deepak Kapoor

AMSTA-AR-CCH-A, B65, Mark Nicolich

AMSTA-AR-AET-M, B355, Kenneth Willison (5 copies)

AMSTA-AR-CCL-BD, B65, Thomas Ziza

AMSTA-AR-ET, B172, Robert Scola (2 copies)

76. U.S. ARMY BENET WEAPONS LABORATORY, CCAC, Armament

Research, Development and Engineering Center, Armament, Munitions and Chemical Command, ATTN: SMCAR-CCB-TL, Watervliet, NY 12189-5000

77. U.S. ARMY, Building 172, Picatinny Arsenal, NJ 07806

G. Obrien

78. U.S. ARMY, Building 172, Picatinny Arsenal, NJ 07806

R. Price

79. U.S. ARMY CENTER FOR HEALTH PROMOTION AND PREVENTIVE MEDICINE, USACHPPM, Aberdeen Proving Ground, MD 21010-5422

P. D. Scully

80. U.S. ARMY CENTER FOR HEALTH PROMOTION AND PREVENTIVE MEDICINE, Aberdeen Proving Ground, MD 21010-5422

A. Hillsmeyer

81. U.S. ARMY CENTER FOR HEALTH PROMOTION AND PREVENTIVE MEDICINE, ATTN: MCHB-DC-OHH, 5158 Blackhawk Road, Aberdeen Proving Ground, MD 21010-5422

Maj. McDevitt

82. U.S. ARMY CHEMICAL/BIOLOGICAL DEFENSE AGENCY, Armament, Munitions and Chemical Command, ATTN: AMSCB-CII, Library, Aberdeen Proving Ground, MD 21010-5423

83. U.S. ARMY CORPS OF ENGINEERS, 20 Massachusetts Avenue, NW, Washington, D.C. 20314-1000

R. B. Oswald 
84. U.S. ARMY DEFENSE TECHNICAL INFORMATION CENTER (DTIC), ATTN: DTIC-OCC, 8725 John J. Kingman Road, Suite 0944, Fort Belvoir, VA 22060-6218

85. U.S. ARMY EDGEWOOD RESEARCH, DEVELOPMENT AND ENGINEERING CENTER, ATTN: SCBRD-RTB (Aerodynamics Technology Team), Aberdeen Proving Ground, MD 21010-5423

86. U.S. ARMY ENVIRONMENTAL POLICY INSTITUTE, Georgia Institute of Technology, 430 Tenth Street NW, Suite S-206, Atlanta, GA 30318-5768

R. Kangari

87. U.S. ARMY MATERIAL COMMAND, 5001 Eisenhower Ave., Alexandria, VA 22333-0001

J. C. Hill

88. U.S. ARMY MATERIAL COMMAND, ATTN: AMCSG-H, 5001 Eisenhower Ave., Alexandria, VA 22333-0001

Maj. Leggieri

89. U.S. ARMY MATERIAL SYSTEMS ANALYSIS ACTIVITY, ATTN: AMXSY-MP Aberdeen Proving Ground, MD 21010-5423

90. U.S. ARMY MEDICAL COMMAND, ATTN: MCHO-CL-W, 2050 Worth Road, Ft. Sam Houston, TX 78234

E. Daxon

91. U.S. ARMY MEDICAL RESEARCH DETACHMENT, ATTN: MCMR-UWW, LTC 2800 Q Street, Building 824, Wright-Patterson Air Force Base, OH 45433-7947R

Everett Langford

92. U.S. ARMY PRODUCTION BASE MODERNIZATION ACTIVITY, Building 172, AMSMC-PBR, Dover, NJ 07801-5001

D. W. Yee

93. U.S. ARMY RESEARCH LABORATORY, ATTN: AMSRL-MM, Aberdeen Proving Ground, MD 21005-5069

Mr. Robert Dowding

94. U.S. ARMY RESEARCH LABORATORY, ATTN: AMSRL-OP-CI-B, Technical Library, Aberdeen Proving Ground, MD 21010-5423 
95. U.S. ARMY RESEARCH LABORATORY, ATTN: AMSRL-WT, Aberdeen Proving Ground, MD 21005

Dr. William deRosset

96. U.S. ARMY RESEARCH OFFICE, P.O. Box 1221, Research Triangle Park, NC 27709-2211

Dr. Andrew Crowson

97. U.S. ARMY RESEARCH OFFICE, P.O. Box 12211, Research Triangle Park, NC 27709-2211

Dr. Kailasam Iyer

98-99. U.S. ARMY SYSTEM LIFE CYCLE READINESS OFFICE, Heavy Metals Management Office, Picatinny Arsenal, NJ 07806-5000

ATTN: AMSTA-AE-SRE, B172, Kenneth Gold

AMSTA-AE-SRE, B172, Michael Los

100-101. U.S. ARMY TANK MAIN ARMAMENT SYSTEM, Picatinny Arsenal, NJ 07806-5000

ATTN: SFAE-ASM-TMA-PA, B171A, Edward Kopacz

SFAE-ASM-TMA-PA, B171A, Vincent Rosamilia

102. U.S. ARMY TRADOC ANALYSIS COMMAND-WSMR,

ATTN: ATRC-WSS-RS White Sands Missile Range, NM 88002

103. UNIVERSITY OF DENVER, Department of Sociology, Denver, CO 80208-0209

T. E. Drabek

104. UNIVERSITY OF TEXAS AT AUSTIN, Department of Civil Engineering, Austin, TX 78712-1076

C. M. Walton 